\title{
Meeting targets : tools to support integrated assessment modelling of global change
}

Citation for published version (APA):

Janssen, M. A. (1996). Meeting targets : tools to support integrated assessment modelling of global change. [Doctoral Thesis, Maastricht University]. Universiteit Maastricht. https://doi.org/10.26481/dis.19961129mj

Document status and date:

Published: 01/01/1996

DOI:

10.26481/dis.19961129mj

Document Version:

Publisher's PDF, also known as Version of record

\section{Please check the document version of this publication:}

- A submitted manuscript is the version of the article upon submission and before peer-review. There can be important differences between the submitted version and the official published version of record.

People interested in the research are advised to contact the author for the final version of the publication, or visit the DOI to the publisher's website.

- The final author version and the galley proof are versions of the publication after peer review.

- The final published version features the final layout of the paper including the volume, issue and page numbers.

Link to publication

\footnotetext{
General rights rights.

- You may freely distribute the URL identifying the publication in the public portal. please follow below link for the End User Agreement:

www.umlib.nl/taverne-license

Take down policy

If you believe that this document breaches copyright please contact us at:

repository@maastrichtuniversity.nl

providing details and we will investigate your claim.
}

Copyright and moral rights for the publications made accessible in the public portal are retained by the authors and/or other copyright owners and it is a condition of accessing publications that users recognise and abide by the legal requirements associated with these

- Users may download and print one copy of any publication from the public portal for the purpose of private study or research.

- You may not further distribute the material or use it for any profit-making activity or commercial gain

If the publication is distributed under the terms of Article $25 \mathrm{fa}$ of the Dutch Copyright Act, indicated by the "Taverne" license above, 


\section{Meeting Targets}

\section{Tools to Support Integrated Assessment Modelling of Global Change}


złggts T gnits9luh 


\title{
Meeting Targets
}

\author{
Tools to Support \\ Integrated Assessment Modelling \\ of Global Change
}

\section{Proefschrift}

ter verkrijging van de graad van doctor aan de Universiteit Maastricht,

op gezag van de Rector Magnificus, Prof.mr. M.J. Cohen volgens het besluit van het College van Dekanen,

in het openbaar te verdedigen

op vrijdag 29 november 1996 orn 14.00 uur

door

Marcus Alexander Janssen

geboren te Hendrik-Ido-Ambacht in 1969 
Promotoren:

Prof.dr.ir. J. Rotmans

Prof.dr.ir.drs. O.J. Vrieze

Leden van de beoordelingscommisie:

Prof.dr. H.J.M. Peters (voorzitter)

Prof.dr.ir. W.E. Bijker

Prof. H. Dowlatabadi (Carnegie Mellon University, USA)

Prof. C.S. Holling (University of Florida, USA)

Prof.dr. L. Hordijk (Landbouw Universiteit Wageningen)

(C) Marco Janssen

Lay-out \& cover design: Esther Mosselman

Edited by: Judith Jaffe, M.A.

CIP-GEGEVENS KONINKLIJKE BIBLIOTHEEK, DEN HAAG ISBN 90-9009908-5 


\section{Contents}

$\begin{array}{ll}\text { Preface } & \text { ix }\end{array}$

1. INTRODUCTION

1.1 The Issue $\quad 2$

1.2 Scope and Objectives 4

1.3 Structure of the Dissertation 5

2. GLOBAL CHANGE 7

2.1 Introduction $\quad 8$

2.2 The Global System. 8

2.2.1 The Human System $\quad 8$

2.2.2 The Issues within the Human System 11

2.2.3 The Environmental System 13

2.2.4 The Issues within the Environmental System 17

2.3 Integrated Assessment $\quad 21$

$\begin{array}{ll}2.3 .1 \text { Introduction } & 21\end{array}$

2.3.2 Integrated Assessment Models: A General Description 23

2.3.3 Limitations and Possibilities of Integrated Assessment Models 24

2.3.4 Integrated Assessment Modelling: Methods and Tools 25

2.3.5 Integrated Assessment Models of Global Climate Change 27

2.4 Setting the Scope 34

3. METHODOLOGICAL ISSUES 35

3.1 Introduction 36

\begin{tabular}{ll}
3.2 Optimization $\quad 39$ \\
\hline
\end{tabular}

3.2.1 Introduction 39

3.2.2 Optimization Applied to Global (Climate) Change $\quad 40$

3.2.3 Applications in this Dissertation 41

3.3 Perspectives $\quad 42$

3.3. I Uncertainty in Perspective 42

3.3.2 Cultural Theory 43

3.3.3 Applications in this Dissertation $\quad 47$

3.4 The Evolutionary Modelling Approach 49

3.4.1 The Evolution of Modelling 49

3.4.2 Complex Adaptive Systems $\quad 50$

3.4.3 An Evolutionary Integrated Modelling Approach 52

3.4.4 An Integrated Modelling Paradigm $\quad 55$

3.4.5 Applications in this Dissertation $\quad 57$ 
4. OPTIMIZATION OF A MATHEMATICAL SYSTEM OF GLOBAL CLIMATE CHANGE

4.1 Introduction $\quad 60$

4.2 The Model OMEGA 62

4.2.1 DICE: Dynamic Integrated Model of Climate and the Economy 62

4.2.2 IMAGE 1.0: An Integrated Model to Assess the Greenhouse Effect 65

4.2.3 OMEGA: An Optimization Model for Economic and Greenhouse Assessment

4.3 Optimization Methodology $\quad 69$

4.3.1 Problem Formulation 69

4.3.2 Sequential Reduced-System Programming 70

4.3.3 Some other Optimization Approaches 81

4.4 Analysis. $\quad 86$

4.5 Results $\quad 87$

4.5.1 Optimal Responses $\quad 87$

4.5.2 Performance Results 91

4.5.3 Comparing Results of OMEGA. with DICE 93

4.6 Conclusions 102

Appendix 1: Reduced version of OMEGA 103

Appendix 2: Performance Results for all Experiments non- $\mathrm{CO}_{2} \mathrm{BAU} \quad 105$

Appendix 3: Performance Results for all Experiments non- $\mathrm{CO}_{2} \mathrm{AP} \quad 106$

5. TARGETS 107

5.1 Introduction 108

5.2 TARGETS Modelling Paradigm 110

5.2.1 The Integrated Systems Approach 110

$\begin{array}{ll}5.2 .2 \text { Conceptual Framework } & 112\end{array}$

5.2.3 Aggregation Level 116

5.2.4 Calibration and Validation $\quad 117$

5.3 Horizontal Integrative Description of TARGETS 118

5.3.1 Pressure Models 118

5.3.2 State Dynamics Models $\quad 119$

5.3.3 Impacts Models $\quad 119$

5.3.4 Response Models $\quad 120$

5.4 Vertical Integrative Description of TARGETS 120

5.4.1 The Global Population and Health Model $\quad 120$

5.4.2 Energy/Economics Model 1.22

5.4.3 The Global CYCLES Model 124

5.4.4 The Global TERRA Model $\quad 126$

5.4.5 The Global Water Model AQUA 128

5.5 Interlinkages between Submodels of TARGETS $\quad 130$

5.6 Retrospective 131 
6.1 Introduction 134

6.2 Bottom-up versus Top-down Modelling $\quad 135$

6.3 Methodology 136

6.4 Four Global Energy Scenarios 137

6.5 Climate Change Constraints 145

6.6 Delayed Response $\quad 150$

6.7 Hedging Strategies 155

6.8 Integrated Policy for Climate Change and A.cidification 159

6.9 Conclusions 161

7. THE INITIAL ALLOCATION OF EMISSION RIGHTS 163

7.1 Introduction 164

7.2 Historical Regional $\mathrm{CO}_{2}$-Emissions 166

7.2.1 Introduction 166

7.2.2 Fossil Fuel Combustion $\quad 166$

7.2.3 Land Use Changes 168

7.3 Emission Debts $\quad 170$

7.3.1 Historical Contribution to $\mathrm{CO}_{2}$ Concentration Rise $\quad 170$

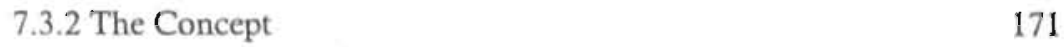

7.3.3 The Results 175

7.4. Emission Rights 179

7.4.1 The Allocation Model 179

7.4.2 Emission Rights Allocation Based on Uniform Parameter Distributions 181

7.4.3 Emission Rights and Perspectives $\quad 183$

7.4.4 Scenarios of Perspective Change 191

7.5 Conclusions 194

Appendix: Summary of Assumptions in the IPCC 1992 Alternative Scenarios 196

8. THE BATTLE OF PERSPECTIVES 197

8.1 Introduction 198

8.2 The Concept 200

8.3 The Model 202

8.4 Modelling Learning and Adaptive Behavior 206

8.4.1 Introduction 206

8.4.2 World View 206

$\begin{array}{ll}\text { 8.4.3 Management Style } & 209\end{array}$

8.4.4 Evaluating Perspectives $\quad 212$

8.5 Exploratory Experiments 215

8.5.1 Introduction 215

8.5.2 Utopias and Dystopias 215

8.5.3 Changing Perspectives 220

8.5.4 Surprises 229

8.6 Conclusions 232 
9.1 Introduction $\quad 234$

$\begin{array}{ll}9.2 \text { Malaria } & 237\end{array}$

$\begin{array}{ll}9.2 .1 \text { Introduction } & 237\end{array}$

9.2.2 The Human Host $\quad 238$

9.2.3 The Parasite $\quad 238$

9.2.4 The Mosquito 238

9.2.5 The Environment 239

9.2.6 Climate Change Impacts 239

9.2.7 The History of Modelling Malaria 240

9.2.8 Modelling Resistant Development 241

9.3 Modelling Malaria: A Complex Adaptive Systems Approach 242

9.3.1 Introduction 242

9.3.2 The Malarial System $\quad 242$

9.3.2.1 The Mosquito Population 245

9.3.2.2 The Human Population $\quad 247$

9.3.3 Adaptation Modelling 249

9.3.3.1 The Mosquito 250

9.3.3.2 The Parasite 253

9.3.3.3 Migration and Refugees among Mosquitoes and Parasites 254

9.4 The Experiments 254

9.4.1 Introduction 254

9.4.2 Impact of Control Programs 259

9.4.3 Sensitivity of Malaria Incidence to Migration 262

9.4.4 Sensitivity of Malaria Incidence to the Coverage Rate 264.

9.4.5 Sensitivity of Malaria Incidence to Temperature Change 265

9.4.6 Sensitivity of Malaria Incidence to Initial Resistance 267

9.4.7 Sensitivity of Malaria Incidence to the Operators of the GA 268

9.4.8 Adaptive Malaria Management 269

9.5 Conclusions and Discussion $\quad 272$

10. CONCLUSIONS 275

10.1 Major Findings 276

10.2 Future Research Avenues 281

$\begin{array}{ll}\text { References } & 285\end{array}$

$\begin{array}{ll}\text { Samenvatting } & 303\end{array}$

$\begin{array}{ll}\text { Curriculum vitae } & 307\end{array}$ 


\section{Preface}

Like any book, this dissertation is a compromise between the original aspirations of the author and the realities of publication. It can be viewed as the result of an evolutionary process. I started with the aim to develop an optimization study of the TARGETS model, the development of which was just beginning when I started as. a Ph.D. student. Due to delays in the ambitious goals of the TARGETS model, and, although I served as a model integrator for two years, I had to shift my objectives.

Especially the influence of Mitchell Waldrop's book Complexity: The Emenging Science at the Edge of Order and Chaos has made me change the direction of my research. While supporting the development and integration of the various parts of the TARGETS model, I became aware that, without exception, existing models in the field fail to tackle various crucial parts of the problem, such as the gap between the economists and natural scientists, the fact that current integrated assessment models do not deal with the adaptive behavior of biological and social agents, and the notion of various perspectives among the agents as a way to explicate the many uncertainties surrounding global change. The dissertation, therefore, became a box of ideas of applying new methodological developments on various aspects of integrated assessment modelling.

The title "Meeting Targets" refers to the optimization approach and policy supporting tools as employed in this dissertation. Furthermore, it refers to the TARGETS model, which was under development during my membership of the Global Dynamics and Sustainable Development group. Working in the TARGETS team inspired me in doing this Ph.D.

In writing this dissertation, I have greatly benefitted. from the knowledge of and support from many colleagues from the Dutch National Institute for Public Health and the Environment (RIVM) and colleagues from other institutes and universities. First, I would like to thank the RIVM which gave me the opportunity to perform this project. Next, I thank my professors Jan Rotmans and Koos Vrieze for the intellectual freedom they gave me in doing my interesting research. I thank Bert de Vries for his enthusiastic, critical, but stimulating attitude towards my research activities and his help in: performing the experiments with his energy model as described in Chapter 6. I am also grateful to "professor" Jodi de Greef, for sharing his knowledge and for having provoking and clarifying discussions on complexity and complex systems. I thank Pim Martens for his enthusiastic cooperation in applying evolutionary modelling techniques on his malaria model as described in Chapter 9.

Furthermore, thanks to all other members of the Global Dynamics and Sustainable Development project, the former Center of Mathematical Methods of the RIVM, the IMAGE team, the M development team, and the Bureau for Environmental Assessments of the RIVM.

Special thanks to Esther Mosselman who helped with the lay-out of this book and the cover design, and to Judith Jaffe, who edited the manuscript.

Finally, I would like to thank my parents, brother, family and friends. Special thanks for the running mates of Hellas in supporting me successfully in meeting other targets. 
1

Introduction

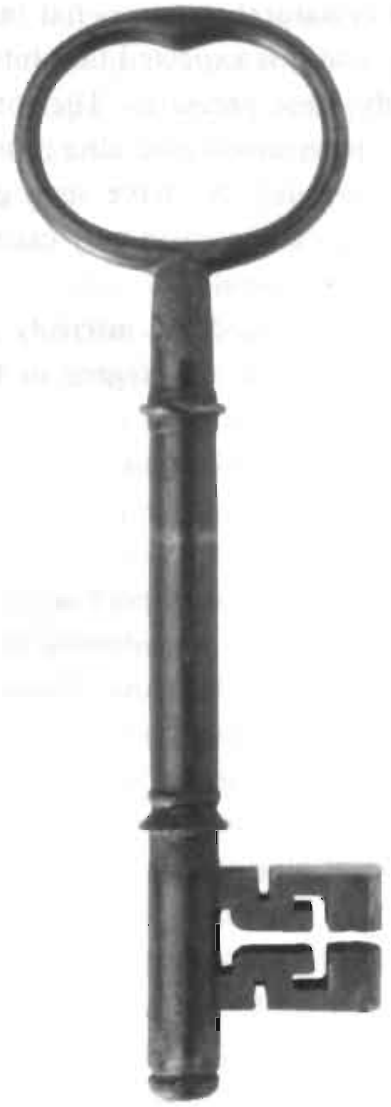




\section{O-}

MEeting TARgets

\subsection{The Issue}

Human activities have always modified the natural environment. The emergence of the integrated world economy, during the last two centuries, has especially increased both the intensity and scale of these modifications. Our use of land, water, minerals, and other natural resources has increased more than ten fold during the past two centuries, and it is expected that future increases in population and development will intensify these pressures. The totality of changes on planet Earth, including all human interventions and alterations, is considered as gl 5 bal change. During the last few decades, we have seen growing acceptance of the insight that the increase in intensity and scale may cause serious harm, leading to increasing risks to human health and wealth.

The fast rate, the global scale, and the intensity of current human-indtced changes have not earlier been seen to this degree in history, although concerns about limits to human development have been discussed in earlier times. Plato, for example, lamented in his Critias that agricultural activities had transformed the land of Attica. More recently in the history of mankind, almost two centuries ago, Malthus (1798) saw food production as a land-limited resource that could not possibly be increased quickly enough to keep pace with a growing population. The present wave of environmental concerns was stimulated by important publications such as Rachel Carson's Silent Spring (1962) and Limits to Growth by Meadows et al. (1972) and got its place on the international agenda of policy makers with the Brundtland Report (WCED, 1987) and the United Nations Conference on Environment and Development in Rio de Janeiro in 1992.

These policy efforts aim at stimulating sustainable development. Although there is no common definition, a widely accepted interpretation is that of a development meeting the needs of all present population without compromising the ability of future generations to meet their own needs. There are various kinds of approaches in which science may support policy-making in order to stimulate sustainable development. An approach which became popular after World War II is the use of mathematical models.

Analytical mathematical models have been used in physical sciences for centuries, but due to the rapid development of the computer, mathematical models are being used in a broader range of disciplines to support decision-making. These models can be used to order and analyze complex systems; the insights derived from the mathematical models may improve our perception of the complex reality. 
Mathematical models have been used to support policy since World War II. The 1950's and 1960's were a relatively stable period of economic growth in the Western World. Technology could seemingly handle any difficulty that came along. Affluence was seen as growing and permanent, and the standard of living, it was believed, would continue to improve for individuals and generations. The world was a place of simple equilibria, linear-responses were proportionate to stimuli, and policies could match this linearity. Cost-benefit analysis, optimization, and econometric models seemed to be quite appropriate.

During the 1960 's, the image of a stable society changed, due to changes in the intellectual, psychological, and artistic views of the younger generation, accelerated by the Vietnam War, the murder of John F. Kennedy and the renewal of ecological concerns. The oil-crises in the 1970's led to a change in the western economic success-story. During these decades, we became more aware that reality can show strongly nonlinear behavior and that social, technological, economic and ecological systems may be surprisingly sensitive to internal and external fluctuations, as well as perturbations. Recent examples of surprising changes are the drastic economic, social and political changes in the former Soviet Union and the countries of Central and Eastern Europe, and the nationalistic civil wars in those areas, such as in former. Yugoslavia.

Humankind is currently confronted with problems of uncommon complexity. The intensity and scale of the problems have increased, and one has become aware of the unpredictable nonlinear behavior of the global system. Due to the scale of human activities, current decisions about which pathways to follow in the next century are of crucial importance for the well-being of the next generations. More and more, we are becoming aware, on one hand, of our limited understanding of the global system, and, on the other hand, of the interdependence of the various activities and consequences in time, place and scale. Intensified efforts by our scientific community are needed to pass on to decision-makers the information and insights which are useful to help to balance the risks, while meeting targets for a. sustainable development.

A recent development in the efforts to support policy-making and the scientific: community is the development of integrated assessment models. The multi- and interdisciplinary approach of "integrated assessment" endeavors to keep track of how the pieces of the puzzle fit together. Integrated assessment models are one type of tool to support integrated assessment and aim at integrating simplified versions of expert models, so that users can scan the future in an interactive way. But it is not. the predictions, the glimpses of the future derived from the electronic oracles, which are of main interest. Because the future is inherently unpredictable, such forecasts: 
are of limited use in decision-making. Nevertheless, mathematical models may enrich our insights into the behavior of complex reality. The improvements of our perception of reality may help us to improve our decision-making. It is this notion which is an underlying assumption of the usefulness of the tools which are presented in this dissertation.

\subsection{The Scope and Objectives}

Since 1992, I have been a member of the Global Dynamics and Sustainable Development Program of the National Institute of Public Health and the Environment (RIVM). The main objective of this multi- and interdisciplinary project is to render applicable the concept of sustainable development from a global perspective (Rotmans et al., 1994). In order to achieve this, a global, integrated assessment model entitled TARGETS (Tool to Assess Regional and Global Environmental and health Targets for Sustainability) is being developed (Rotmans and De Vries, 1996). TARGETS is intended to be used to perform an analysis and assessment on a global scale of social and economic processes, biophysical processes, and effects on ecosystems and humans from an integrated system dynamics perspective. Such a global, integrated assessment model can yield insights into the complex interrelations in time and space between increasing worldwide pressure on the environment, pollution, and disruption of essential biogeochemical cycles, and the effects of anthropogenic influences on the environment, human health, and society as a whole, in the longer term.

During the development of the TARGETS model, I became aware of the embryonic state of the methodology of integrated assessment modeling. Tools for old problems are used within this new research area; I believe that not all of these tools are equally satisfactory. It might be that the new problems need new tools in order to support decision-making in a more satisfactory manner. The existing tools probably result more wrong questions being asked, rather than in giving the right insights. This can be explained by what is called the streetlight effect: a drunk who has lost his door key looks for it under the street lamp because that is the brightest spot. Many of the tools currently used deeply bias the way modellers perceive the world and, thus, influence the contents and shapes of models: "If the only tool you have is a hammer, you tend to treat everything as if it were a nail", Maslow (1966).

It is not my intention to claim to provide the right or the best tools. Existing tools are examined and new methodological approaches are applied to various aspects of the problem of global change. In this way, I hope to contribute to the 
development of more suitable tools by appropriately reshaping existing methods for the new kind of problems which have been recognized during the last decades.

More concretely, I will discuss three methodological issues which are of importance within the present integrated assessment models or are expected to be of importance in the next generations of those models. First, a widely spread modelling paradigm within the integrated assessment modelling community, especially in economic oriented models, is the use of optimization. Those models use simple linearized representations of the global system, excluding important processes. I believe that such models are of limited use, as the inclusion of processes is essential in modelling global change. Therefore, I will analyze the sensitivity of the present optimization approach and provide a new direction of optimization oriented studies in integrated assessment modelling by applying heuristic search algorithms on advanced simulation models.

Secondly, in current modelling efforts, uncertainty is represented as a statistical artifact using educated guesses of ranges of parameter values. As a moderate step to improve the consistency of educated guesses and to make uncertainty explicit, I propose to use plural qualitative and quantitative descriptions of reality to explain partly the uncertainty rooted in subjective judgment. Some illustrative case studies are performed on quantifying various perspectives of reality.

Thirdly, the present generation of integrated assessment models is dominated by a mechanistic world view. Studies often aim to understand the mechanisms to control the system. I believe that this view is limited in understanding the ever changing, evolving, and adapting global system. An evolutionary modelling approach is proposed as a useful alternative, regarding some exemplary parts of the global system as an organism, instead of a machine. By applying, recent developments in mathematics and computer science on evolutionary modelling, some case studies are worked out to illustrate the possible benefits of such an approach.

\subsection{Structure of the Dissertation}

In the next Chapter, the field of global change is discussed by giving an overview of the main issues of the changing global system. Furthermore, an overview is given of the emerging field of integrated assessment. Especially methodological concerns in the integrated assessment community are discussed, as well as prominent integrated assessment models in the field of global climate change. 
The methodological backbone of this dissertation is presented in Chapter 3. It discusses a number of methodological problems of integrated assessment modelling. A general discussion is given about what methodological contributions are made in this dissertation; these are worked out in Chapters 4 - 9.

As an illustration of the problems in the present use of optimization, I have applied optimization to a mathematical dynamic system of global climate change in Chapter 4. Various optimization routines are used, including a new algorithm: sequential reduced-system programming. In Chapter 6, exploratory optimization oriented experiments have been applied with the TARGETS model, which is presented in Chapter 5. The experiments mainly focus on global energy policies meeting climate change targets, taking account of the uncertainties in economic and technological development. Especially the consequences of delayed responses are illustrated.

The initial allocation of emission rights is discussed in Chapter 7, in which perspectives are used to quantify subjective uncertainties in weights of "equal" sharing. Furthermore, the egalitarian concept of emission debts is presented.

Two case studies are worked out, focusing on adaptive behavior of social agents, as well as adaptation of biological agents. Chapter 8 presents a study which aims at modelling learning and adaptive behavior of social agents, a concept which is called: the Battle of Perspectives. An example is worked out for the global climate change problem. A malaria case-study in Chapter 9 deals with modelling adaptation of mosquitoes and parasites. Special attention is given to development of resistance and adaptation of these agents to climate change.

After the concluding remarks, the dissertation closes with a discussion of possible future avenues for the near future of integrated assessment modelling of global change. 


\section{2}

Global Change

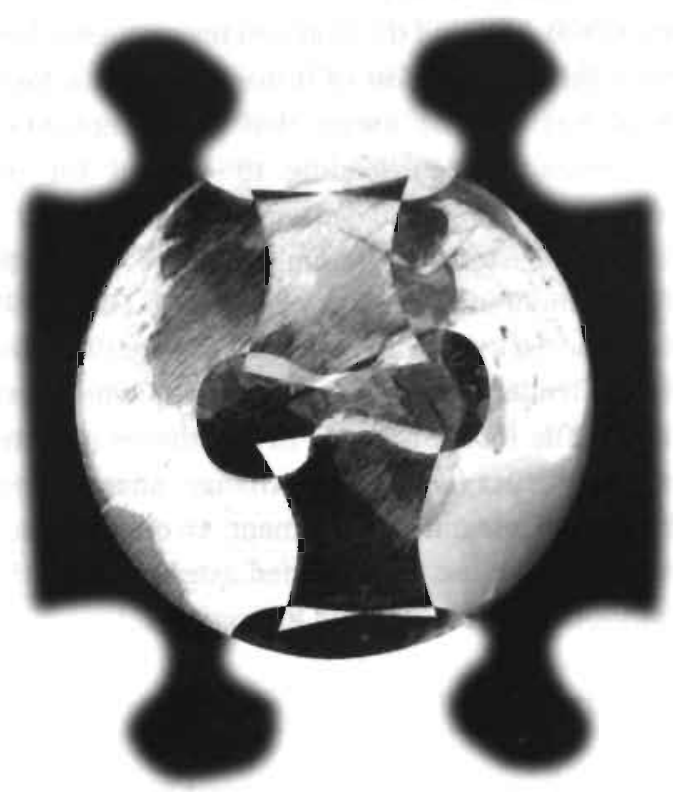




\subsection{Introduction}

The history of humankind is a continuing record of interactions between peoples' efforts to improve their well-being and the environment's ability to sustain these endeavors. Environmental constraints led to innovations and social development, as well as social stagnation and human suffering. Several ancient cultures collapsed because the increase of affluence and population led to an over-exploitation of the natural environment (Ponting, 1992); some examples are: the Mesopotamians $( \pm 2000 \mathrm{BC})$, the Mayas (800 AD) and the Polynesians on Easter Island (16th century). While the interactions throughout most of history were on a local scale, during the last decades, mankind has become aware that the complexity and scale of the interactions have increasingly been leading to a need for new environmental management. People became aware of various new threats for mankind, such as climate change, acid rain, ozone depletion, resource exhaustion, limits to the availability of food and unpolluted fresh water. In this Chapter, I will give a brief overview of the state-of-the-art knowledge of global change. This overview is meant to show the non-informed reader the range of problems which may be analyzed in assessing global change. The tools discussed in this dissertation will only be applied on a small subset of these issues (e.g. climate change, energy, and malaria), and are applied within the context of integrated assessment, an emerging scientific field within the global change research. The use of integrated assessment, with a special focus on integrated assessment modelling, is discussed in Section 2.3.

\subsection{The Global System}

I will briefly describe the human and environmental system and related issues based on a number of state-of-the-art publications on the various subjects. This is meant to give a rough overview of the various issues related to global change. I have based the overview on e.g. Clark and Munn (1986), UNEP (1992), WHO (1992b), White et al. (1992), Graedel and Crutzen (1993), Seitz (1995) and Brown (1995; 1996).

\subsubsection{The Human System}

\section{Population and Health}

About $8000 \mathrm{BC}$, there were about 5 million people in the world; this grew to about 200 million in about $1 \mathrm{AD}$, and 500 million in 1650 . The recent period of rapid population increase began in the eighteenth century, growing, from a population size of 1000 million in 1800 to more than 5000 million now. Projections of the United 
Nations show expectations of more than 10 billion people at the end of the next century. This can be explained by changes in demographic and health stages generally described in the theory of health transition (Frenk et al., 1993; Ness et.al., 1993; Bongaarts, 1994; Niessen et al., 1996). It describes how populations can go through typical demographic and health stages when they change from living in pre-industrial conditions to having a mortality pattern that is found in the post-modern societies (Omran, 1971) (see Figure 2.1). The early stages are characterized by high fertility levels and a low life expectancy and an epidemiological pattern of infectious diseases. In the last recognized stages, low levels of fertility are dominant, sometimes even below replacement level, life expectancy is high, and diseases are postponed until the last phases of life. The health transition is defined as including all these changes, as well as the changes in the provision and organization of social and health-related services. The changes in fertility and mortality often have different timing, leading to a rapid population growth in the first half of the demographic transition. Also specific sub-populations may find themselves in varying stages of these two processes, depending on their socio-economic and environmental conditions of life. It is assumed that there is a convergence of developmental directions, as societies shift from agricultural-oriented societies to modern industrial economies.

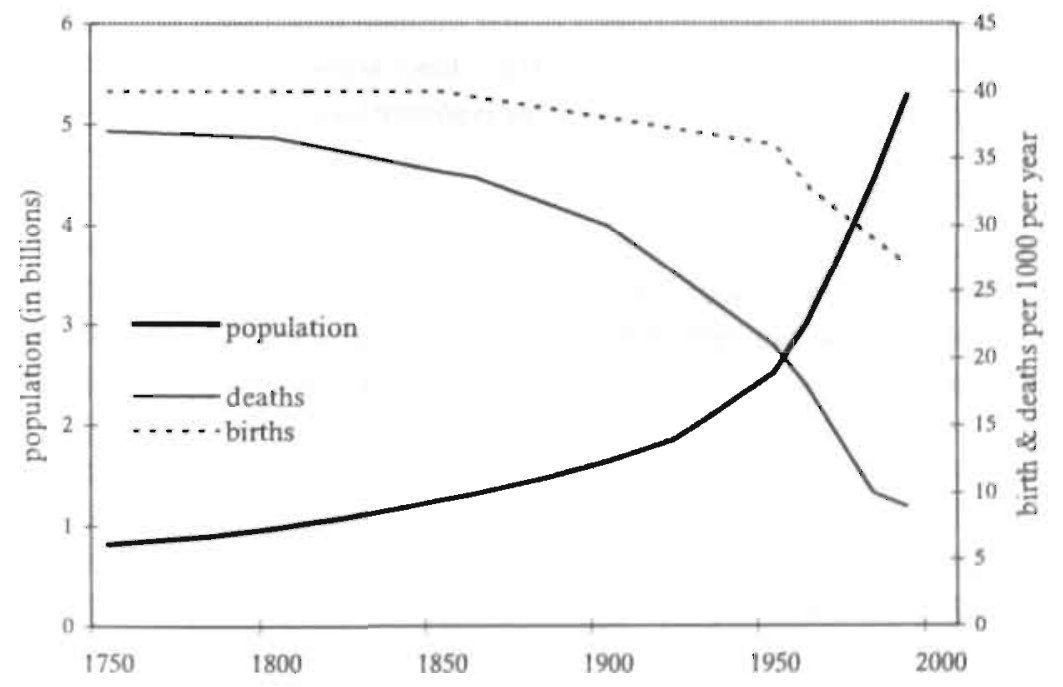

Figure 2.1: The demographic transition at the global level. 


\section{Economic Activities}

The growth of population and populations' activities caused an emergence of a "world economy" about two centuries ago. Demands for many goods and services are more and more met by supplies from all over the world, and this trend continues to increase. The volume of the world trade has risen the past two hundred years by a factor of about 500, leading to a share of one-third of world's total GNP. The emergence of a global market dominated by many large corporations illustrates the globalization of economic activities. The expanding world economy consists of a number of interrelated human activities that exert pressures on the total environment. Three types of activities are distinguished: agriculture, industry, and services.

\section{- Agriculture}

Humanity has relied solely on hunting, fishing, and gathering food for most of its two million years of existence. Since agriculture appeared about 10,000 years ago, human activities have caused significant changes in land use. In the last century, the mounting demand of growing populations led to an accelerated change in agricultural practices. Increasingly, fragile land has been cultivated more intensively, resulting in environmental damage, especially land degradation. Resources from outside the agricultural system have been imported (water through irrigation, chemical pesticides and fertilizers, fossil fuels energy). This has allowed increased agricultural productivity, although it has undermined the resource base and caused. environmental problems.

\section{- Industry}

Industrialization has increased personal incomes and material wealth and improved services, particularly transport and communication. However, industrial practices have various side effects such as pollution and resource depletion. To illustrate, I will discuss the energy problem. Before industry developed, human demands for energy were primarily associated with food production. Since some 200 years ago when the industrial revolution started, the energy demand has been increasingly met by fossil fuels replacing renewable resourçes like water and biomass. Since 1900, world use and production of energy has increased more than tenfold. During the first half of this century, energy production slackened during the First and Second World Wars and the depression of the 1930's. In the 1950's and 1960's, the economy grew rapidly. The inexpensive oil and gas were the basic fuels, supporting the economic expansion. However, during the oil crises in the 1970's, the share of oil and gas was reduced somewhat due to higher prices, in favor of an increasing share of coal. The last decades, people became aware that the use of fossil fuels has a harmful 
impact on the environment, which has led to the desire to limit the use of fossil fuels, by replacing them with traditional alternatives (like biomass and hydropower) or new alternatives (like nuclear and solar energy). Although such a transition might be preferable from an environmental perspective, it is not known what the social and economic consequences will be.

\section{- Services}

The service sector is increasing. The service sector has supported industrial activities by, for example, administration and health care. Nowadays, an increasing part of the National Product is produced by non-material production, such as the booming knowledge sector. The expected emergence of an information society becomes imaginable now that the electronic highway finds its place in the (Westem) world. Although this sector is less material and energy intensive "it requires a higher quality of these resources, which makes the net effect of an increasing service sector on the environment uncertain.

\subsubsection{The Issues within the Human System}

\section{Population and Health}

Socio-economic development largely determines population health levels directly by influencing the immediate surroundings of people, and their health behavior and their access to health services (Niessen et al, 1996). Health standards can be considerably improved in the early stages of the health transition by food availability and good drinking water supply. In the last stage of the transition, the main health determinants are related to life style, like smoking and hypertension.

Population growth leads to increasing pressure on the environment, due to increases in human activities. Environmental factors, in turn, affect both population density, as well as the quality of life of the population. The use of drugs like antibiotics may cause, via the increase of resistance to external pathogenic micro-organisms, a revival of infectious diseases. Climate change may increase the risks of vector-bom diseases like: malaria, schistosomiasis and dengue. Air-pollution affects the health by inhalation of toxic products, while depletion of the ozone layer increases the risk of skin-cancer and cataracts.

Another important question in relation to the health transition is the increasing proportion of elderly people, which will increase the disability ratio and the demand for health services. The transition also leads to an increase in diseases like cancer, Parkinson's disease, and diabetes. 


\section{Poverty and Inequity}

For most of history, a few individuals in many societies had a higher standard of living than their fellow human beings, but the vast majority of people on earth shared a common condition of poverty (Seitz, 1995). The Industrial Revolution created new wealth in the industrialized nations in Europe, which was eventually shared by larger numbers of people. The differences between rich and poor in the world began to increase, where a few nations have achieved their higher living standards by extracting resources from the rest of the world. It is estimated that the difference between the average income of people in rich countries (early industrialized) and poor ("developing") countries was 2 to 1 in 1850,10 to 1 in 1950, and is expected to be 30 to 1 in 2000 (Miller, 1985). Currently, more than a billion people can be classified as being poor, because they lack a standard of living that includes adequate food, safe and sufficient water, sanitation, a secure shelter, and access to education and health care. It is expected that those poor are more vulnerable to global change than others.

\section{Resource Use}

The increasing use of minerals and fossil fuels will lead to depletion of nonrenewable resources. The timing of depletion depends on technological and economic developments. Although fossil fuels are relatively cheap energy supplies to fulfil the energy needs of the growing economies, their use has various side effects. Besides the inequality of acquiring, distributing, and using energy resources, the use of fossil fuels causes serious pressure on the environment. Emissions related to combustion of fossil fuels contribute to the issues of acidification and climate change. In addition to this, land degradation and depletion of resources are important issues. However, suggested alternative energy supplies like biofuels, solar energy, wind, nuclear, hydro-power also have various harmful impacts on the environment. Challenges for the future are to manage the energy demand and balance the energy costs and environmental impact of the various energy supplies.

\section{Extinction of Cultures}

There have been and still are thousands of different cultures on our planet. Each group shares a common history, a common ancestry, and usually a common language and a common religion. They often have common traditions, common ways of doing certain things and of interacting with each other and towards outsiders. Because of these common features that make them different from other peoples, each nation's people see the world and their place in it differently than others, approach problems differently, and have arrived at different solutions to situations human beings face. Extinction of cultures leaves life a little less rich. 


\section{War}

Since World War II, there have been about 150 wars, with $90 \%$ of those occurring in the less developed nations; these wars have killed about 7.5 million soldiers and 14.5 million civilians. The destructive capacity of weapons increase, the number of refugees grows and the ecological damage to the land increases, in a world where people often choose for an armed solution to solve territorial disputes, power struggles, ethnic and religious rivalries or other conflicts.

\subsubsection{The Environmental System}

One way of describing the environmental system is to consider a number of spheres: atmosphere, lithosphere, hydrosphere, and ecosphere. Human interventions within the spheres, especially the disturbances of the biogeochemical cycles, cause a number of issues, as will be discussed in 2.2.4.

\section{Global Systems}

\section{- Atmosphere}

The Earth's atmosphere is a complex system of gases and suspended particles. The composition did not have its origins in the origins of the planet. The atmosphere of today has been derived from the Earth itself by chemical and biochemical reactions. This adaptable system forms a gaseous envelope around the Earth and is a composition of mainly five gases: nitrogen, oxygen, argon, carbon dioxide, and water vapor make up $99.9 \%$ of the total volume of the atmosphere. Human activities cause emissions of various gases and particles which may cause climate change, stratospheric ozone depletion, air pollution, and acid rain.

\section{- Lithosphere}

The lithosphere is the upper zone of the Earth's mantle. The outer boundary forms a complex interface with the atmosphere and hydrosphere. The inner boundary is adjacent to rock, which is near its melting point and is capable of motion relative to the lithosphere above. The processes in the lithosphere, such as solidification, weathering erosion and rising magma, are relatively slow and they involve considerable residence times in the various compartments. Disturbance of the lithosphere consists of changes in element cycles (see below) and the extractions of mineral resources. 


\section{- Hydrosphere}

The processes which bring about changes in phase (water vapor, water, and ice) and the transport of water within the atmosphere are commonly represented by a hydrological cycle. Within the hydrological system, water is stored in the atmosphere $(0.001 \%)$, the land $(1.8 \%)$, the oceans $(96.5 \%)$ and the polar ice caps $(1.7 \%)$. Transfers between stores are effectuated by the processes of evaporation, condensation, precipitation, runoff, and freezing and melting. The greatest exchanges are those between ocean and atmosphere. Human activities cause pollution and disturbances in the availability of fresh water. Furthermore, climate change and groundwater extraction may cause sea levels to rise.

\section{- Ecosphere}

At the top of the lithosphere, throughout the hydrosphere and intor the lower atmosphere, lies a transition zone, which contains and is assumed to be created by living organisms. Life far outweighs its relatively small mass in the significance of its effect on the constitution of the lithosphere, hydrosphere, and atmosphere. An essential element in the ecosphere are the biogeochemical cycles.

\section{Biogeochemical Cycles}

Living organisms extract chemicals from their environment, hold on to them for a period, and lose them again. In its natural biogeochemical cycles, a mineral often goes through a reservoir stage, which can be gaseous, liquid, or sedimentary (see Figure 2.2a-d). Below, I discuss four of the basic element cycles: carbon (C), nitrogen (N), phosphorous (P), and sulphur (S). More detailed discussions can be found in, for exanaple, Lovelock (1979), Bolin and Cook (1983), Begon et al. (1990), Schlesinger (1991), Butcher et al. (1992), White et al. (1992), Anderson et al. (1993) and Den Elzen et al. (1995)

\section{- The Carbon Cycle}

Carbon is the most important element for life on Earth. Carbon dioxide in the atmosphere is used by green plants in the process of photosynthesis and is returned to the atmosphere from plant respiration. Carbon is also passed along the food chain and is converted into carbon dioxide by decay processes. The carbon cycle is not only part of the terrestrial web of life, but also a part of the aquatic web as carbon dioxide diffuses in and out of both fresh and salt water. In the ocean, photosynthesis is confined to the light zone, where a large proportion of carbon becomes bound in shells and exoskeletons of ocean invertebrates as calcium carbonate. When these organisms die, their body coverings may be buried in the sediment and are, thereby, 


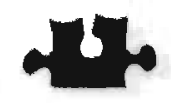

isolated from biological activity. Through geological time, some of these carbon deposits become part of coral reefs or limestone rock and some are slowly transformed into fossil fuels.

The carbon cycle is currently disturbed by fossil fuel combustion and deforestation, leading to increased atmospheric $\mathrm{CO}_{2}$-concentrations which may contribute to a climate change.

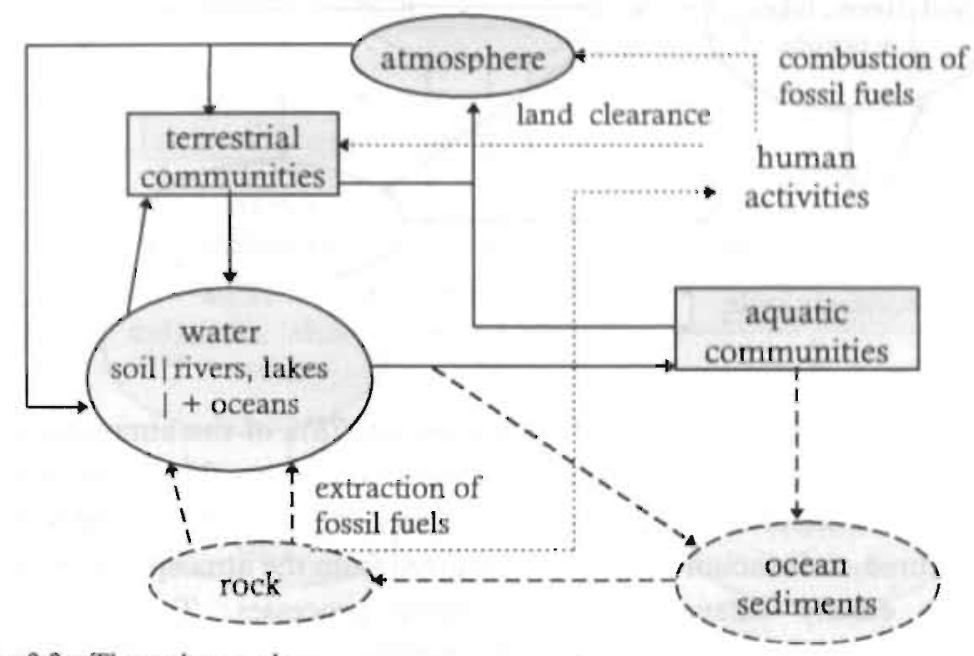

Figure 2.2a: The carbon cycle.

\section{- The Phosphorus Cycle}

The phosphorus cycle does not involve an atmospheric phase, since the primary reservoir of this element is rock or sediment. As rocks erode, phosphate is released into the environment. Plants then absorb phosphate through their roots. Organisms acquire this essential element in food and excrete any excess in faeces. When phosphate enters streams and lakes in large amounts, it can act as a stimulant to algae growth and eventually usurp the oxygen that other forms of water life require (eutrophication). 


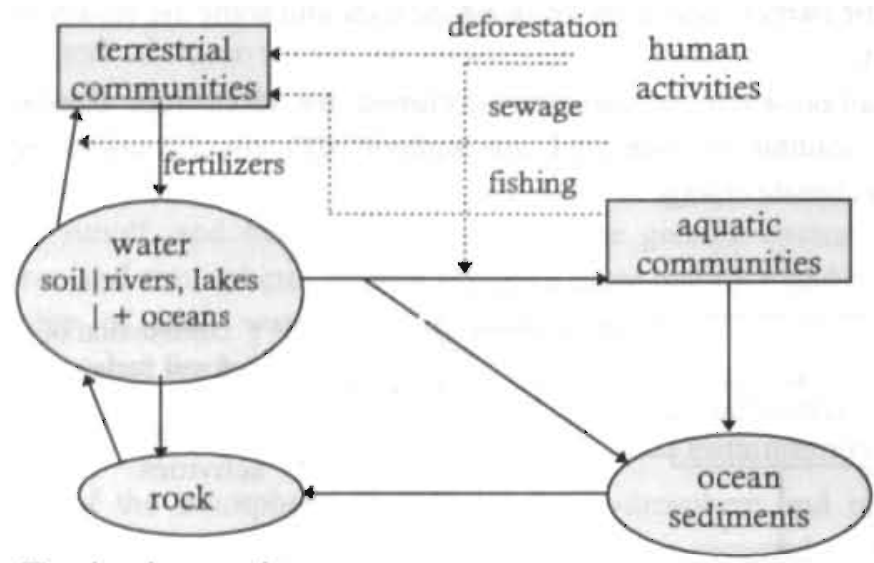

Figure 2.2b: The phosphorus cycle.

\section{- The Nitrgeer, furle}

Even though nitrogen makes up approximately $78 \%$ of the atmosphere, it is not utilized by most living organisms in its atmospheric form $\left(\mathrm{N}_{2}\right)$. Life processes generally require nitrate or some other nitrogen compound. Before nitrogen fertilizers were manufactured, the amount of nitrogen removed from the atmosphere by natural processes was closely balanced by denitrifying processes. Today, industrial intervention in the nitrogen cycle appears to fix more nitrogen than can be denitrified. These additional amounts of nitrogen in the soil often disrupt natural balances, resulting in large run-offs of nitrogen compounds into rivers, streams, and lakes.

Figure 2.2c: The nitrogen cycle.

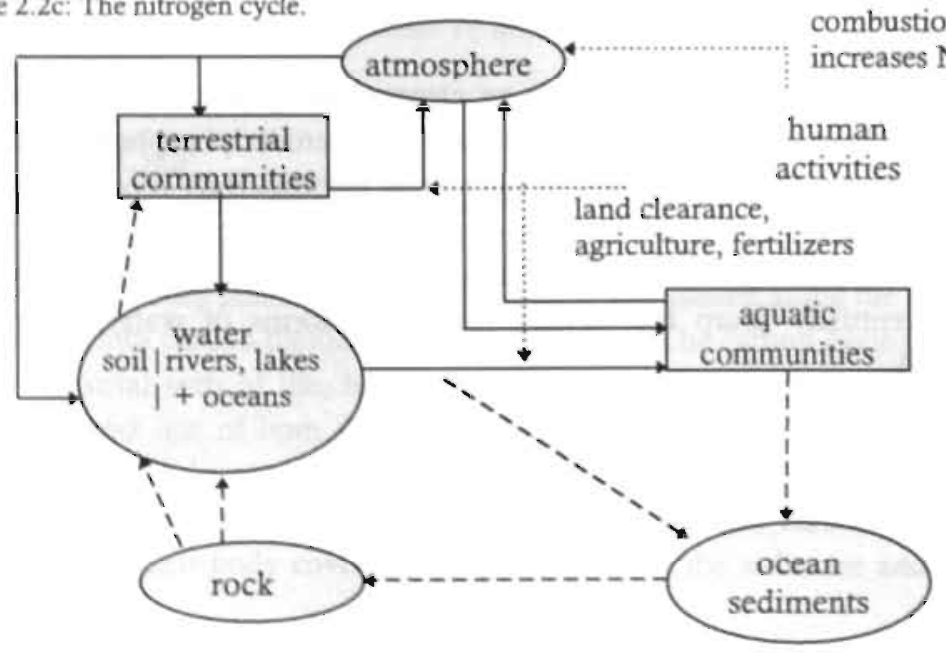




\section{- The Sulphur Cycle}

The sulphur cycle involves both an atmospheric and a sedimentary phase. In nature, sulphur exists in several states. It is used in the manufacture of many products, including chemicals, matches, and fertilizers. As a constituent of coal and petroleum products, sulphur causes atmospheric contamination when its products are burned, which contributes to the acidification and climate change.

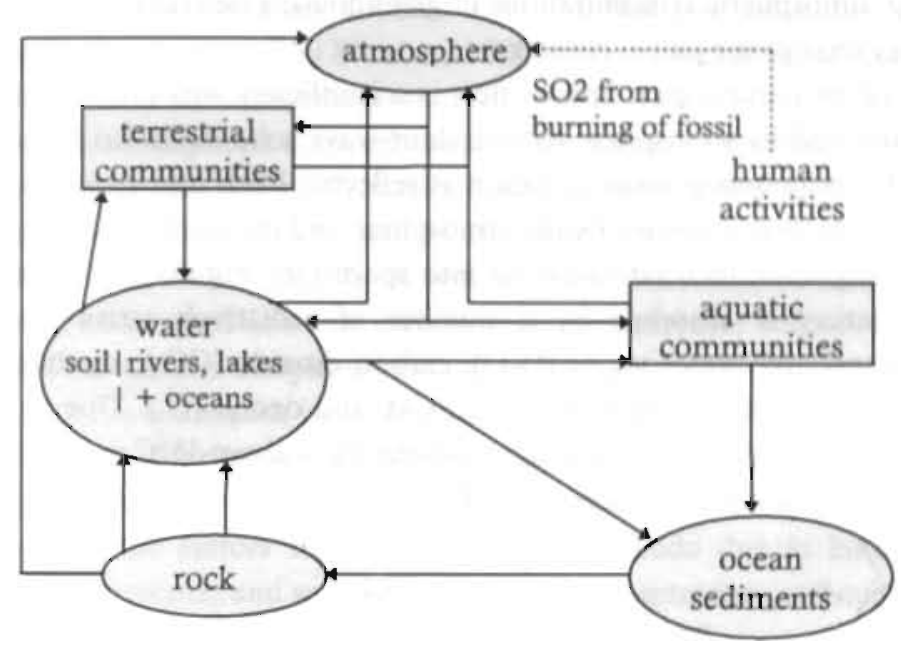

Figure 2.2d: The sulphur cycle.

\subsubsection{The Issues within the Environmental System}

Human activities cause a lot of changes in the environment. Pollution of water; land, and air results. Reduced availability' of clean water, clean and fertile soils, and clean air leads to more harm to human health and wealth and to damage to the wellbeing of other species. Many species have already become extinct; various areas have already been ruined by human activities, the Aral Sea being only one of them. The expected growth of population, economic activities, land use, resource depletion, pollution may lead to unexpected consequences. Our knowledge of our Earth system is insufficient to project the results of the "experiment" of humankind. In this dissertation, I will mainly focus on climate change. Therefore, I will restrict the discussion of environmental issues to climate change and the interlinked issues of stratospheric ozone depletion and acid rain. 


\section{Climate Change}

Tyndall (1863) had suggested that small changes in atmospheric composition could alter the climate and Arrhenius (1896) estimated that a doubling of carbon dioxide would raise global mean temperature by $4^{\circ} \mathrm{C}$ to $6^{\circ} \mathrm{C}$. However, it was not until the mid-1970's that any attention was paid to this environmental issue. At present, due to the observed changes of the composition of the atmosphere, there is growing concern that increasing atmospheric concentrations of greenhouse gases (like $\mathrm{CO}_{2}, \mathrm{CH}_{4}, \mathrm{NO}_{x}$ and CFCs) may change the global climate.

The theory of the natural greenhouse effect is scientifically well established (IPCC, 1990). The Earth and its atmosphere receive short-wave solar radiation from the sun. About $30 \%$ of the incoming solar radiation is reflected back into space, mainly by clouds. The remainder is absorbed by the atmosphere and the Earth's surface, before it is emitted as long-wave thermal radiation into space (see Figure 2.3). Most of this long-wave radiation is absorbed by a number of radiatively-active trace gases (greenhouse gases), like water vapor $\left(\mathrm{H}_{2} \mathrm{O}\right)$, carbon dioxide $\left(\mathrm{CO}_{2}\right)$, methane $\left(\mathrm{CH}_{4}\right)$, nitrous oxide $\left(\mathrm{N}_{2} \mathrm{O}\right)$, chlorofluorocarbons (CFCs), and ozone $\left(\mathrm{O}_{3}\right)$. Due to the heat trapping of these gases, the Earth's surface temperature is about $15^{\circ} \mathrm{C}$ instead of $-18^{\circ} \mathrm{C}$, which is called "the natural greenhouse effect".

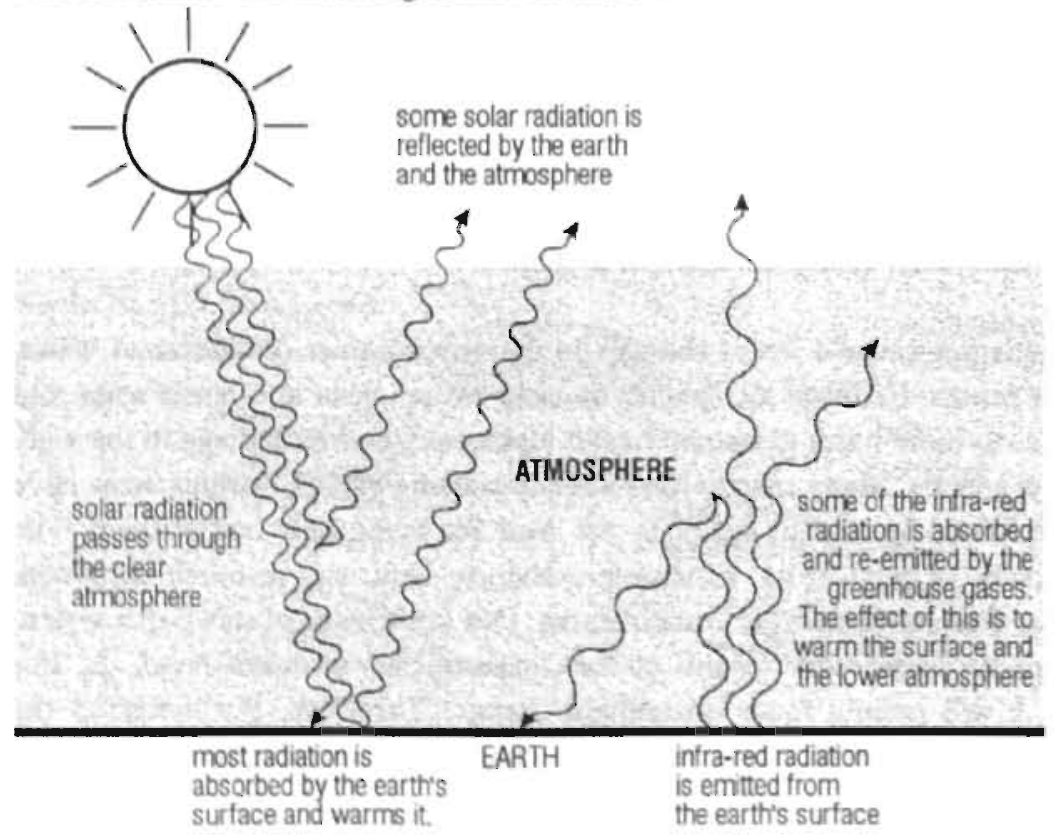

Figure 2.3: A simplified diagram illustrating the greenhouse effect (IPCC, 1990). 
During the past centuries, the atmospheric concentrations of the greenhouse gases have increased. Carbon dioxide increased steadily from $280 \mathrm{ppmv}$ (around 1800) to 354 ppmv in 1990 (IPCC, 1990). Observation of the isotopic dilution of the atmosphere and surface oceans with respect to radiocarbon and stable carbon isotopes. gives strong evidence of the anthropogenic source of the concentration increase. Concentrations of other greenhouse gases have also been increased: methane concentration has more than doubled over the last two centuries, nitrous oxide concentration has increased by $8 \%$ since 1800 , while new greenhouse gases like CFCs have been added to the atmosphere since the 1930 's.

Increases in atmospheric concentrations of the radiatively active gases, as observed over the past decades, will undoubtedly lead to a net increase in energy input to the lower stratosphere, and, hence, will be succeeded by elevation of the global mean surface temperature of the Earth. However, many uncertainties exist concerning to what extent, where, and how fast such temperature increases will occur and what the impact will be on the biosphere, including human society. Scientific evidence of climate change is a complex issue, because of our limited understanding of the global system and since observations are influenced by various natural factors influencing the climate system such as, for example, volcanic activities, fluctuations in solar activity, and anthropogenic factors such as variations in albedo due to land use changes, changes in tropospheric and stratospheric ozone concentration, and sulphur emissions from industry.

Our limited understanding of the global system is illustrated by various issues which are discussed briefly below:

- Climate forcing by sulphate aerosols: The radiative effects of sulphate aerosols, derived from emissions of sulphur dioxide and from biomass burning, have only recently been acknowledged to affect the radiation balance, both directly and indirectly. Averaged worldwide, aerosol cooling may partly offset greenhouse warming, although this is a gross oversimplification, since because of their short atmospheric lifetimes the effects exhibit large spatial and temporal differences. First modelling results show regions with a warming and a cooling effect, if forcing by sulphate aerosols and greenhouse gases are combined (Taylor and Penner, 1994; Wigley, 1995; Mitchell et al., 1995; IPCC, 1996).

- Indirect climate forcing by CFCs. Halocarbon-induced stratospheric ozone depletion can, likewise, directly affect climate change via negative radiative forcing due to ozone depletion (Ramaswamy et al., 1992; WMO, 1992). At present, globally and annually averaged ozone forcing is setting off $80 \%$ of the total, direct, globalmean radiative forcing caused by the halocarbons under considerations. 
- Geophysical feedbacks, various physical processes in the atmosphere-oceancryosphere system directly modify the response to radiative forcing, such as water vapor formation, cloud feedback, and snow-ice albedo feedbacks.

- Biogeochemical feedbacks. influence of uptake of emissions of greenhouse gases, affection of atmospheric chemical condition, by, for example, the $\mathrm{CO}_{2} / \mathrm{N} / \mathrm{P}$ fertilization effects, eutrophication effect, plankton-related feedbacks, soil acidification, etc..

If climate change should occur, it might also cause various impacts, such as droughts (desertification), sea level rise, shifts in the growing zones for vegetation (both natural and agricultural), and changes in the availability of fresh water, increased risks of vector-born diseases and heath-stress. Not all changes are harmful. Some countries could eventually benefit from increased rainfall or longer growing periods in the present marginal areas. However, the international community is generally concerned with the impacts of climate change and wants to minimize the risks of damage.

\section{Ozone Depletion}

Molina and Rowland (1974) warned that the use of CFCs might damage the ozone layer, which might lead to increases in UV-B radiation, which might cause a multitude of negative effects, such as: augmentations in the incidence of skin cancer and other diseases, reduction of crop yields, and damages to materials. The measurements of the Antarctic ozone gap, and the middle- and high latitude ozone losses suggest the seriousness of this environmental problem (Farman et al., 1985; WMO, 1992).

The main ozone-depleting gases are CFCs and other halocarbons, which are currently in use as refrigerants aerosol, propellants, and solvents. Due to the industrial production of $\mathrm{CFCs}$, the atmospheric chlorine $(\mathrm{Cl})$ levels have increased from about 0.6 ppbv up to about 3.9 ppbv (Fraser et al., 1992). Increased chlorine levels are the major cause of the observed ozone losses over the last decades, although also other man-made and natural causes exist. The concern about the depletion of the stratospheric ozone layer led to the Montreal Protocol in 1987, which was followed by Amendments in London (1990) and Copenhagen (1992), which should finally lead to a phasing-out of a large group of halocarbons by the year 2000 .

An important feedback is the decreasing stratospheric temperature (due to climate change) which slows down the rate of ozone destruction. Furthermore, the increased UV-B radiations have a negative feedback impact on biological production 
(phytoplankton growth). Due to ozone depletion, these gases also have an indirect climate change impact (Ramaswamy et al., 1992; WMO, 1992).

\section{Acidification}

A concern about acidification damage (e.g. declines in fish populations and ultimate extinctions in rivers and lakes) was first voiced by Svante Odén in Sweden (Odén, 1968). Important gases involved in the production of acid deposition are nitrogen dioxide $\left(\mathrm{NO}_{2}\right)$, nitrous oxide $(\mathrm{NO})$, sulphur dioxide $\left(\mathrm{SO}_{2}\right)$, and ammonia $\left(\mathrm{NH}_{3}\right)$, which can be transported by winds for distances of up to 1000 kilometers. The major causes of emissions are the burning of fossil fuels and industrial processes. The forest decline in Europe became a major public issue in the early 1980's, which led to effective measures to reduce the acid emissions, although the deposition levels still exceed the target levels which are necessary to prevent economic and ecological damage in large parts of Europe and North America. Furthermore, due to industrial development, acidification has become a problem in other places of the globe, especially in Southeast Asia.

\subsection{Integrated Assessment ${ }^{\dagger}$}

\subsubsection{Introduction}

There is a growing interest in an integrated approach to global change, called integrated assessment. This can be subscribed to three streams (Rotmans and De Vries, 1996). First of all, there is a growing awareness of the rapidly increasing complexity of global change, encompassing a diversity of research topics which all seem to be interconnected in one way or another. An "end-to-end" approach may then foster better understanding and insights into this complicated matter. Secondly " global change is beset with many uncertainties, many of which are fundamental by nature. The sources and types of these uncertainties, however, may range widely. An overall analysis helps in comprehensively analyzing and comparing the various sources and types of uncertainty. An integrated approach may pave the road towards a full quantification, as well as cumulation of the crucial uncertainties in the global system. Thirdly, reductionistic methods which consider only parts of global change in isolation, have not been successful so far in proving a comprehensive picture of the consequences of a accelerated global change.

\footnotetext{
1 This Section is mainly based on Rotmans and Dowlatabadi (1996) and Rotmans and De Vries (1996).
} 
The term integrated assessment is not new, albeit its use is growing rapidly. Rotmans and Dowlatabadi (1996) define integrated assessment as an interdisciplinary process of combining, interpreting and communicating knowledge from diverse scientific disciplines in such a way that the whole cause-effect chain of a problem can be evaluated from a synoptic perspective with two characteristics: (i) it should have added value compared to single disciplinary oriented assessment, which means that integrated assessment endeavors to keep track of how the pieces of the puzzle fit together, indicating priorities for the narrower disciplinary research; (ii) it should provide useful information to decision-makers, which means that integrated assessment offers an opportunity to develop a coherent framework for consideration of multiple objectives of decision-making and identification of possible policy criteria. Adopting this exclusive interpretation of integrated assessment for consideration of global change means that the various causes, the mechanisms, and the impacts of global change should be addressed.

Integrated assessment is an iterative, continuing process, where, on one hand, integrated insights from the scientific community are communicated to the decisionmaking community and, on the other hand, experiences and learning effects from decision-makers form the input for scientific assessment. This complex, intuitive, and value-loaded process can not be performed by only one, unique approach. Therefore, multiple, diverse approaches to integrated assessment are needed, varying from formal and experimental methods, such as models to heuristic and exploratory methods such as expert judgment or policy exercises. The numerous approaches used at this time to address the global change issue from an integrated viewpoint are all relatively immature. These immature efforts vary in their emphasis and in the ensemble of methods employed to achieve integration and assessment. The differences in emphasis are a direct consequence of the different perspectives people have, leading to different problem orientations of these efforts. The divergence of methods employed arises from the uneven state of data and theory across different problem domains covered by integrated assessments.

Different kinds of tools can be used to perform an integrated assessment (Rotmans and Dowlatabadi, 1996): (i) integrated assessment models, aspect-compart models or expert models; (ii) simulation-gaming and game-theoretic concepts; (iii) scenarioanalysis; (iv) qualitative assessments.

In this dissertation, I aim to appropriately reshape existing methods as tools which may support integrated assessment modelling of global change. Therefore, I will discuss integrated assessment modelling in more detail. 


\subsubsection{Integrated Assessment Models: A General Description}

Among the tools for integrated assessment are integrated assessment models. Current projects in integrated assessment modelling elaborate on a tradition that was founded in the early 1970's by the Club of Rome (Meadows et al., 1972, 1974). Over the past 20 years, numerous global models have been built (Brecke, 1993), all of which were rather complex, highly aggregated and only partially integrated. The next generation of integrated assessment models was developed with focus on the acid rain issue.

The RAINS model (Alcamo et al., 1990), which was developed and used to address the contentious issue of acid rain in Europe, was one of the more successful among these earlier studies. More recently, the challenge of global climate change has prompted the development of a new flight of models (see also Section 2.3.5), such as the IMAGE 1.0 model (Rotmans, 1990); the DICE model (Nordhaus, 1992); the PAGE model (Hope et al., 1993); and ICAM (Dowlatabadi and Morgan, 1993b). The development of a new generation of integrated assessment models of global (climate) change is now underway; one promising model in this field, TARGETS, is discussed in more detail in Chapter 5.

In general, integrated assessment models try to describe quantitively as much as possible of the cause-effect relationship of a phenomenon and of the cross-linkages and interactions between different issues. More specifically, integrated assessment models of global change are designed to analyze the phenomenon of global change from a synoptic perspective. Although there are some serious attempts being made to construct an integrated model of the Earth's atmosphere, hydrosphere, and terrestrial biosphere (Fisher, 1988; Krapivin, 1993), it is conceptually and technically not yet possible to link, let alone integrate, a variety of complex, detailed and threedimensional models. Therefore, it is often more appealing to make use of reduced form models or metamodels for each component of the integrated assessment framework, which are small enough to be comprehensible, flexible, and easily linked to one another. A metamodel is a simplified, condensed representation of a more complicated and detailed model (expert model), which provides approximately the same behavior as the expert model from which it is extracted. There are various methods for developing metamodels, which vary in complexity, ranging from fully parameterized models to process-oriented models. The simplicity principle applied to metamodels within an integrated assessment framework is also justified by the everlasting deficient state of scientific knowledge. However, the primary prerequisite for these metamodels is that they should be tested, verified, calibrated, and validated thoroughly against expert models and observational data. Interlinking a variety of 
metamodels requires the definition of one single conceptual framework, affording harmonization with respect to aggregation level, temporal, and spatial scales.

The challenging aspect of building such an integrated assessment model is to find the right balance between simplicity and complexity; aggregation and realistic outcomes; stochastic and deterministic elements qualitative and quantitative linkages; transparency and uncertainty. Furthermore, it is fundamentally important to face the limitations of the model to be built to recognize the type of issues/questions that cannot be addressed by and what falls beyond the scope of the model.

\subsubsection{Limitations and Possibilities of Integrated Assessment Models}

Integrated assessment models of global change may not pretend to offer a comprehensive picture of all relevant processes of complex reality. In view of the accumulation of uncertainties, which is inherent to integrated assessment modelling, the interpretative and instructive value of an integrated assessment model is far more important than its predictive capability, which is limited by the incomplete science upon which it is constructed. Therefore, rather than a prediction tool, an integrated assessment model is an interpretative tool, whereby its predictive value is rather low.

According to Rotmans and Dowlatabadi (1996), limitations and drawbacks of integrated assessment models are: (i) too complex a structure; (ii) high level. of aggregation level; (iii) explication of counter intuitive results; (iv) inadequate treatment of uncertainty; (v) absence of stochastic behavior; (vi) limited verification and validation; and (vii) inadequacy of knowledge and methodology.

Thus, in view of the above, we must learn from the experiences with the past generation of integrated assessment models. According to Rotmans and Dowlatabadi (1996), the major advantages of integrated assessment models are:

(i) An integrated approach enables the inclusion of systems interactions and feedback mechanisms and can, therefore, yield insights that scatted information cannot offer. Such a model can provide useful indications of the potential range and magnitude of global phenomena and of the scale of the interventions which are necessary to prevent or mitigate symptoms of global change.

(ii) The simplified nature of modules in integrated assessment models permit rapid prototyping of new concepts and exploration of its implications. If the interactions between various systems are well characterized, this rapid prototyping may indicate means of validation for the new concept. Furthermore, if the model is being used as a decision-analysis tool, the rapid prototyping will permit exploration of the implication of the new finding or concept on policy choice, and the value of pursuing this new concept (from purely applied policy science implications). In this way, these models 
are instruments which can help us keep track of and manage our knowledge and insights into the present and future driving forces behind our complex, social, economic, and ecological structures.

(iii) Uncertainties, crucial lacunae in current scientific knowledge and weaknesses in discipline-oriented expert models can be identified and revealed. Furthermore, accumulation of uncertainties can be analyzed and interpreted.

(iv) These models are outstanding means of communication between scientists and exponents of all kinds of disciplines; they also foster communication between scientists and decision-makers.

\subsubsection{Integrated Assessment Modelling: Methods and Tools}

\section{Aggregation}

One of the critical issues in integrated assessment modelling is that of aggregation versus disaggregation. The level of aggregation within a modelling framework refers to the formulation of the dynamics in the model in terms of complexity, level of detail, which is often closely related to the spatial and temporal resolution chosen within the framework. The problem with integrated assessment models is that they consist of a variety of submodels, which have different aggregation levels, in other words, which differ in complexity, spatial, and temporal resolution, etc. In many cases, these submodels are only linked, but not integrated. The different levels of temporal and spatial aggregation in the submodels of an integrated assessment framework result in an "hourglass" structure to the model. The economic-energy models operate in multiyear time steps on large-nation or regional political boundaries. Atmospheric chemistry models operate in small time steps on a small scale, while climate models have a relatively coarse spatial resolution, grid-cells of a few degrees, but run at a fine temporal resolution. Finally, ecological impact models require data at fine spatial resolutions of about half a degree, but their time resolutions vary greatly from one day to a season or a year.

\section{Calibration and Validation}

There are many definitions and interpretations of the terms calibration and validation. Moreover, complete calibration of system earth's models is impossible because the underlying systems are never closed (Oreskes et al., 1994). Within the integrated assessment modelling community, calibration is usually defined as the procedure for comparing model results with measurements of the real system (historical output, for instance observational data). The question to answer is how close the model approximates the observational data. 
Validation is defined here as the procedure for testing the adequacy of a mathematical model. Validation can be subdivided into two different types. The first is practical validation, which concerns the validity of the outcomes of the model. In fact, this implies that the outcomes of the model are compared with observational data. The major difference with calibration is that the comparison should be based on a new set of data, which lies outside the calibration pathway. The second type of validation is conceptual validation, which concerns the test of whether the model represents the real system. This implies the internal structure of the model is tested, by testing whether the concepts and theoretical laws of the system under consideration are interpretated and represented in a sound way.

\section{Uncertainty}

Because integrated assessment models are intended as a means of capturing the entire cause-effect chain of global change, such models are prone to an accumulation of uncertainties. The issue of uncertainty is, therefore, of crucial importance. Doing uncertainty analysis for integrated frameworks is rather difficult, because of the accumulation of uncertainties, but also because of the different types and sources of uncertainty.

Several approaches to analyze and present uncertainties are possible: (i) specifying a set of future scenarios; (ii) sampling method, assuming probability distributions for numbers of inputs.

A crucial issue in the communication of uncertainty to decision-makers is to make the uncertainties explicit, visible and tangible. Therefore, we need a classification of different sources of uncertainty. Morgan and Henrion (1990) distinguish uncertainty about empirical quantities, uncertainty about the functional form of models, and disagreement among experts, whereas Funtowitcz and Ravetz (1989) distinguish technical, methodological, and epistemological uncertainties.

\section{Visualization}

Because of the level of complexity of the structure of integrated assessment models and the results these models produce, it is of crucial importance to use appropriate visualization techniques. The use of visualization techniques enables the presentation of information that corresponds to the way people intuitively perceive images. Such features help to make the model backgrounds as well as the model results more immediately available and broadly accessible to a wide range of users, which may vary from the general public to decision-makers. In fact, modelling and visualization are interwoven and should, therefore, be integrated. Apart from the traditional and mostly static techniques to visualize information such as diagrams, graphs, tables, and 


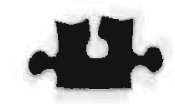

histograms, innovative techniques which allow for an interactive, comprehensive and dynamic presentation of information, can be used. These visualization techniques can help: (i) to make models as transparent as possible; (ii) to make the underlying theories as clear as possible; (iii) to provide easy-to-use interfaces; (iv) to display uncertainties and complexity of the systems behavior comprehensibly.

Taking into account a barrier which may be formed by the high degree of abstraction and level of complexity of the messages that will have to be brought across to decision-makers, visualization can form an alternative for aggregation.

\subsubsection{Integrated Assessment Models of Global Climate Change}

A number of approaches to integrated assessment modelling of climate changes exist. Each approach has strengths and weaknesses relative to the others. Moreover, each modelling team has chosen to focus on different aspects of the climate change issue. A classification of the approaches often used is the distinction in policy evaluation models or process-oriented models, and policy optimization models (Rotmans and Dowlatabadi, 1996; Weyant et al., 1996). The first approach emphasizes the physical, ecological, economic, and social consequences of policies, where the latter optimizes over key policy control variables given formulated policy goals.

Although the models are mixtures of various modelling paradigms, I have clustered them by the dominating paradigm (Table 2.1). I distinguish: the optimization approach, the systems dynamics approach, and the deterministic and stochastic simulation approach (the approaches will be discussed in Chapter 3). 
Table 2.1: Summary Characterization of Integrated Assessment Models (Based on Rotmans and Dowlatabadi, 1996)

\begin{tabular}{|c|c|c|c|c|c|c|c|}
\hline Model & $\begin{array}{l}\text { Forcings: } \\
0 . \mathrm{CO}_{7} \\
\text { 1. other } \\
\text { GHG } \\
\text { 2. acrosols } \\
\text { 3. land use } \\
\text { 4. other }\end{array}$ & $\begin{array}{l}\text { Geographic } \\
\text { Specificity } \\
0 \text {. global } \\
\text { 1. continental } \\
2 \text { countries } \\
\text { 3. grids/ } \\
\text { basins }\end{array}$ & $\begin{array}{l}\text { Socio-economic } \\
\text { dynamics } \\
0 . \text { exogenous } \\
\text { 1. economics } \\
\text { 2. tech choice } \\
\text { 3. land use } \\
\text { 4. demographic }\end{array}$ & $\begin{array}{l}\text { Geophysical } \\
\text { simulation } \\
\text { 0. } \Delta \mathrm{F} \\
\text { 1. Global } \Delta \mathrm{T} \\
\text { 2. I-D } \Delta \mathrm{T}, \Delta \mathrm{P} \\
\text { 3. 2-D } \Delta \mathrm{T}, \Delta \mathrm{P}\end{array}$ & $\begin{array}{l}\text { Impact } \\
\text { Asseasment } \\
0 . \Delta T \text { indexed } \\
\text { 1. sea level rise } \\
\text { 2. agriculture } \\
\text { 3. ecosystems } \\
\text { 4. health }\end{array}$ & $\begin{array}{l}\text { Treatment of } \\
\text { uncertainty } \\
\text { 0. none } \\
\text { 1. basic } \\
\text { 2. advanced }\end{array}$ & $\begin{array}{l}\text { Treatment of } \\
\text { decision-making } \\
0 . \text { optimization } \\
\text { 1. simulation } \\
\text { 2. simulation } \\
\text { sith adaptive } \\
\text { decisions }\end{array}$ \\
\hline \multicolumn{8}{|l|}{ Optimization } \\
\hline $\mathrm{DICE}$ & 0,1 & 0 & 1 & 1 & 0 & 1 & 0 \\
\hline RICE & 0,1 & 0,1 & I & 1 & 0 & 1 & 0 \\
\hline CETA & 0 & 0,1 & 1,2 & 1 & 0 & 1 & 0 \\
\hline MERGE 2 & 0,1 & $i$ & 1,2 & 1 & 0 & 1 & 0 \\
\hline \multicolumn{8}{|l|}{$\begin{array}{l}\text { Systems } \\
\text { dynamics }\end{array}$} \\
\hline IMAGE 1.0 & $0,1,3$ & 0 & 0.2 .3 & 1 & 1 & 1 & 1 \\
\hline TARGETS 1.0 & $0,1,2,3,4$ & 0 & $1,2,3,4$ & 1 & $1,2,3,4$ & 2 & 1,2 \\
\hline \multicolumn{8}{|l|}{ Deterministic } \\
\hline IMAGE 2 & $0,1,2,3$ & 3 & $0,2,3$ & 3 & $1,2,3$ & 1 & 1 \\
\hline MiniCAM & $0,1,2,3$ & 2,3 & $1,2,3$ & 3 & 0 & $i$ & $i$ \\
\hline GCAM & $0,1,2,3$ & 2,3 & $1,2,3,4$ & 3 & 0,2 & 1 & $t$ \\
\hline \multicolumn{8}{|l|}{ Stochastic } \\
\hline ICAM 2 & $0,1,2,3$ & 1,2 & $1,3,4$ & 2,3 & $0,1,3$ & 2 & 1.2 \\
\hline PAGE & 0,1 & 1,2 & 1 & 1 & $0,1,2,3,4$ & 2 & 1 \\
\hline
\end{tabular}




\section{The Optimization Approach}

\section{DICE (Dynamic Integrated model of Climate and the Economy)}

DICE is an extended version of a traditional optimal growth model by including a climate sector (Nordhaus, 1992; 1993; 1994). The objective is to maximize the discounted value of utility from consumption. In DICE, population growth and technological change yield productivity growth. Both of these factors of population are exogenously specified and assumed to decline asymptotically to zero.

The single consumer maximizes discounted present value of utility of consumption subject to a Cobb-Douglas production function that includes a climate-damage factor. Emissions per unit output are assumed to decline exogenously at a fixed rate and can be further reduced by costly emission-control measures.

An increasing convex emission-control cost function is estimated from prior studies (Nordhaus, 1991a; 1991b). Current carbon emissions add to atmospheric concentrations via a fixed retention ratio, and realized temperature is modelled by a two-box model representing the atmosphere/mixed-layer upper ocean and deep ocean. Damage from climate change is a quadratic function of realized temperature change with a 3 -degree change calibrated to cause a $1.3 \%$ world GNP loss.

\section{RICE (a Regional Integrated Model of Climate and the Economy)}

RICE is a regionalized version of the DICE model (Nordhaus and Yang, 1995). In the RICE model, the world is divided into a number of regions. Each is endowed with an initial capital stock, population, and technology. The capital market clears through equalization of the real interest rate across regions, where the real interest rate is determined as the weighted average of the marginal products of capital in different regions.

RICE is able to cope with the possibility of different strategies undertaken by nations, and three distinct approaches are worked out: Market (or uncontrolled), Global cooperative, Nationalistic policies.

\section{CETA. (Climate Emissions Trajectory Assessment)}

The CETA suite of models is being developed by Peck and Teisberg (1992; 1993a; 1993b). These models are based on the Global 2100 model of Manne and Richels (1991). Global 2100 combined a macro-economic module of overall economic activity with an energy-technology module. CETA considers only one aggregated region, the world as a whole, instead of several regions as in Global 2100. The focus of the framework is to map out optimal paths of energy investments in the future. Fuel and technology choices are influenced by resource and technology availability as well 
as policies which (through taxes) manipulate relative prices of different fuels. In CETA, reduced form carbon cycles, climate change, and impact modules are included.

MERGE (Model for Evaluating Regional and Global Effects of GHG Reduction Policies)

MERGE is the integrated assessment model which Manne and Richels have developed with additional input from Mendelson (Manne et al., 1994). At the core of this model resides a revised version of the Global 2100, now being exercised to the end of the 22nd century. It embodies a general equilibrium model with 5 world regions, in which each region's consumer makes both savings and consumption decisions. A simple climate model represents atmospheric lifetimes of $\mathrm{CO}_{2}, \mathrm{CH}_{4}$ and $\mathrm{N}_{2} \mathrm{O}$, which yield global changes in radiative forcing, and equilibrium and realized global-average temperature change. Illustrative impact functions are defined separately for market and non-market components. The former is modelled as a quadratic function of realized temperature change fitted to a single judgment point estimate. The latter estimate is a willingness to pay in each region to avoid a specified temperature change that is a logistic function of regional income, in effect modelled as a worldwide public good.

\section{The Systems Dynamics Approach}

\section{IMAGE 1.0 (Integrated Model to Assess the Greenhouse Effect)}

IMAGE 1.0 is a model which links models from various scientific areas with policies for controlling global climate change (Rotmans, 1990). The model is meant for developing and evaluating long-term climate strategies; it calculates, on the basis of historical and future emissions of greenhouse gases, the global temperature and sea level rise. In IMAGE 1.0, the climate change problem is modelled as a dynamic system with discrete steps of half a year and a simulation period of 200 years, from 1900 to 2100 . IMAGE 1.0 itself is a concatenation of autonomously functioning models (modules): a world energy/economy model, atmospheric chemistry model, carbon cycles model, climate model, sea level rise model, an UVB-impact model and a socio-economic impact model for the Netherlands. 
TARGETS (Tool to Assess Regional and Global Environmental and Health Targets for Sustainability) ${ }^{2}$.

Besides the IMAGE model, a second shoot of RIVM's integrated assessment program is the integrated assessment framework TARGETS (Rotmans et al., 1994; Rotmans and De Vries 1996), which aims at the analysis of global change and sustainable development from a synoptic perspective. The model works at exploring the long-term dynamics of the human and the environmental system which may shape the Earth system over the next hundred years. The TARGETS framework basically consists of a population and health model, a resource/ economy model, a biophysics model, a land model, and a water model.

All types of model compromise a linkage of pressure, state-descriptive, impact and response module, in this way representing the (vertically) integrated cause-effect chain. On the other hand, by coupling the various pressures, states, impacts and responses for the various subsystems underlying the models, the horizontal integration comes into play. A first version of the TARGETS model has been made operational on a global level. The time horizon of the TARGETS model spans 200 years, starting at the end of the pre-industrial era, supposed to be in 1900, untill the end of the next century, the year 2100 , with time steps varying from one month to one year.

\section{The Deterministic Simulation Approach}

\section{IMAGE 2 (Integrated Model to Assess the Greenhouse. Effects)}

The IMAGE 2.0 model (Alcamo, 1994) evolves from the earlier, global-average model version, IMAGE 1.0 (Rotmans, 1990). The model presents a geographicallydetailed, global and dynamic overview of the linked society-biosphere-climate system, and consists of three fully linked subsystems: the energy-industry system; the terrestrial environment system; and the atmosphere-ocean system. The energyindustry models compute the emissions of greenhouse gases in 13 world regions, as a function of energy consumption and industrial production. End use energy' consumption is computed from various economic driving forces. The terrestrial environment models simulate the changes in global land cover on a grid-scale, based on climatic and economic factors. The role of land cover and other factors are then taken into account to compute the flux of $\mathrm{CO}_{2}$ and other greenhouse gases from the biosphere to the atmosphere. The atmosphere-ocean models compute the build-up of greenhouse gases in the atmosphere and the resulting zonal-average temperature and

\footnotetext{
${ }^{2}$ A more detailed discussion can be found in Chapter 5 .
} 
precipitation patterns. The model includes many important feedbacks and linkages between models in these subsystems.

In order to provide a long-term perspective about the consequences of climate change, the model's time horizon extends to the year 2100. The time steps of the various submodels vary considerably, from one day to five years. In general, the submodels of IMAGE 2 are more process-oriented and contain fewer global parameterizations than previous models.

\section{MiniCAM/GCAM}

The Batelle Pacific Northwest Laboratory runs a program which involves a multidisciplinary team with a long and sustained track record in climate related research. There are two integrated assessment models being developed within the program: MiniCAM and GCAM. The two models are differentiated by their complexity and specificity.

MiniCAM makes extensive use of reduced form modules, characterizing fewer world regions, and fewer economic activities. MiniCAM is specifically designed to be suitable for uncertainty analysis. This framework is composed of the Edmonds-ReillyBams model for projection of economic activity and emissions of greenhouse gases and sulphate aerosols. The MAGICC model (Wigley, 1994) is then used to generate a global temperature response commensurate with the emissions. A model called SCENGEN is then used to arrive at regional climate patterns based on the global climate change. The market and non-market impacts are expressed in economic terms and follow the formulation proposed by Manne et al. (1994).

GCAM is a much more complex framework, making use of detailed models of human activities. The human activities within this framework are simulated using the Second Generation Model (SGM) which is a Calculable General Equilibrium model. Twenty regionally specific models are being developed through collaboration with regional experts. The suite of SGMs are used explicitly to allocate land-use and other resources and estimates anthropogenic emissions. Again, the MAGICC model is used to convert the emissions data to globally averaged temperature and precipitation change, and the SCENGEN model is used to map this change into regional patterns of changed climate according to the output of one or a combination of GCMs. The regional temperature and precipitation fields are employed in detailed regional agriculture, ecology, and hydrology models, in order to assess regional impacts of projected climate change. 
The Stochastic Simulation Approach

\section{ICAM (Integrated Climate Assessment Model)}

The Integrated Climate Assessment Models (ICAM versions 0,1 and 2) were developed by the Carnegie Mellon University, at the Department of Engineering \& Public Policy (Dowlatabadi and Morgan, 1993a; 1993b). The ICAM model versions have brought increasingly sophisticated and detailed descriptions of the climate change problem, at each stage quantifying the uncertainties in the model components and asking where additional research would most contribute to resolution of the climate change policy dilemma. This information is then used in the next iteration of the research program defining the disciplinary research needs and the direction of refinements of ICAM.

The ICAM model versions are designed to capture the uncertainties in knowledge about the precursors, processes, and consequences of climate change. The models can be used to simulate abatement activities, adaptation to a changed climate, and geoengineering activities. The development of ICAM 2 has involved updating all previous modules, development of demographics, fuel market, aerosols, terrestrial ecology and coastal impact modules. The spatial and temporal scales have also been refined to 5 years and 7 geo-political regions. The differentiation between high and low latitudes makes it possible to examine the gross differences in the magnitude of climate change, as well as different economic circumstances and availability of resources needed to adapt to changing climate.

ICAM models have been used to show: the wide range of possible emissions, climate conditions, and impacts, and the dangers of deterministic modelling with narrow sensitivity studies; the importance of aerosol forcing in regional policy decision-making; the relative importance decision rules in policy decision-making; and the relative contribution of the various parts of the problem in making decisionmaking so difficult. Moreover, illustrative runs of ICAM highlight how uncertainties confound the choice of GHG abatement policy, and how key factors in determining the character of the problem and key uncertainties in making informed judgments can be identified.

\section{PAGE (Policy Analysis of the Greenhouse Effect)}

The PAGE model is a probabilistic model with an emphasis on decision analysis (Hope et al., 1993). The model is designed to be simple to use and allows extensive: specification and propagation of uncertainty. PAGE generates abatement and damage: costs estimates for four world regions for the period 1990 to 2100 , and projects: concentrations, radiative forcing and global mean temperature. The added value of 
the PAGE model lies in its treatment of uncertainty. All major parameters on the emissions, atmospheric climate and impacts side are represented by triangula probability distributions whose parameters can be set by the user. These paramete uncertainties are then propagated throughout the model.

\subsection{Setting the Scope}

Global change is a difficult issue to grasp in a transparent and univocal way and the integrated assessment modelling activities have only partly got a grip on this complexity. In the next Chapter, I will discuss some methodological ingredients which may improve integrated assessment modelling of global change; these ingredients are applied in case studies in the Chapters thereafter. 

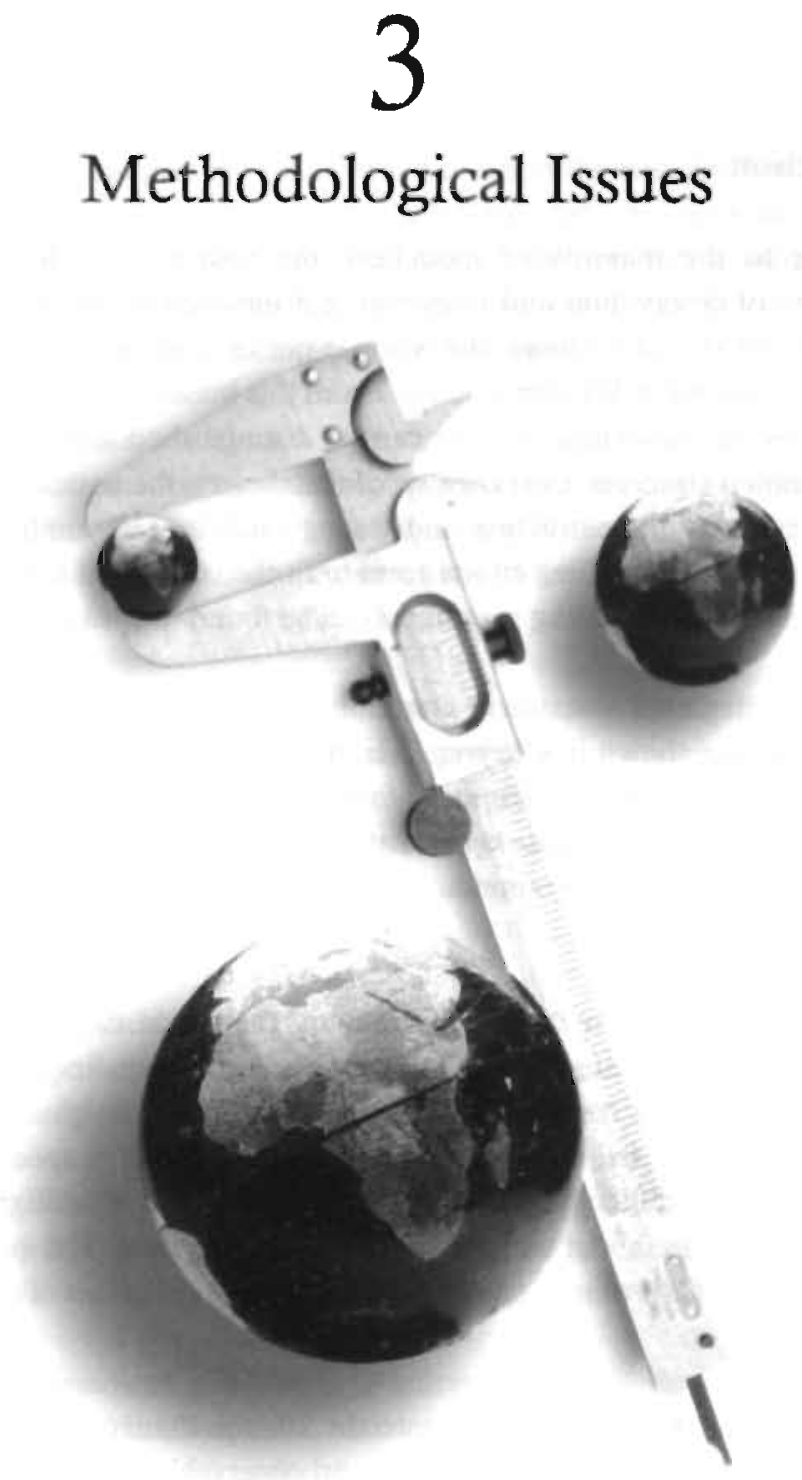


\subsection{Introduction}

According to the majority of modellers, the source of understanding is the rational process of observation and reflection, not intuition or meditating (Meadows and Robinson, 1985). Furthermore, the world is not only knowable, but controllable, a view generally shared in Western culture. From this biased modellers starting point, a number of distinct modelling schools can be distinguished which have, among a number of common concepts, their own set of theories, mathematical techniques, and accepted procedures for constructing and testing models. The implicit assumptions underlying the various modelling efforts seem to fit the concept of a paradigm (Kuhn, 1970): A discussion on modelling paradigms can be found in Meadows and Robinson (1985).

Within the integrated assessment community, various modelling paradigms can be considered as was shown in Chapter 2. In the following. I will briefly discuss the main paradigms which can be commonly found in integrated assessment modelling: system dynamics, econometrics, optimization, and deterministic and stochastic simulation, and I will add a new approach to those: complex adaptive systems.

\section{System Dynamics}

According to the system dynamic paradigm, the world can be described by a conglomeration of interacting feedback loops. By using non-linear equations and time-delays, the stocks of material and information flow through the system. The main efforts are spent on the model structure, while little effort is spent on parameter estimation, which is a difficuil activity because of the inclusion of highly aggregated. parameter and variables, about which there is not enough data. The purpose of most system dynamic models is to study the dynamic long-term behavior of systems:

\section{Econometrics}

Econometricians tend to think in terms of simultaneous equations, linear relations, many exogenous driving variables, and observable statistics. Historical data are rigorously used to determine model parameters, while less time is spent. on specifying the model structure. This model structure is limited by the mathematical requirements of its estimation procedures. The model validity is evaluated by a summary of statistics. The purpose of most econometric models is the precise forecasting of economic variables into the short-term future. 


\section{Optimization}

Optimization techniques are mainly used to select the best decision from a set of clearly-defined alternatives. Any policy problem can be separated into three conceptual components: the objective of the policy, the activities available to achieve it, and the constraints within which those activities must take place. Optimization models are normative, usually linear, static or dynamic.

\section{Deterministic Simulation}

Deterministic simulation models of physical processes consist mainly of sets of differential equations. In such models, the outcome of the operation of the system is fully predicted by the model, so that the precision of the model is only constrained by its realism.

\section{Stochastic Simulation}

Stochastic simulation models try to include explicit uncertainty into the models, using probability distributions for events and/or parameters values. These distributions are based on historical data or educated guesses from scientists. The results are generally presented by depicting the mean together with the reliability intervals.

\section{Complex Adaptive Systems}

Complex adaptive systems include a set of agents and take into account that systems change and organize their component parts to adapt themselves to the problems posed by their surroundings. Because the approach aims at describing, changes in structure and dynamics, it is not the main purpose to make predictions using such models. It may provide us with insights into the behavior of complex nonlinear systems.

Integrated assessment modelling is current being worked out using various modelling paradigms. In Chapter 2, the integrated assesșment models for climate change have been categorized, using the modelling approaches. However, it is likely that the relatively new aspects of global change may not be fully be taken into account by the current modelling paradigms. I will discuss some problems in integrated assessment models and outline approaches which may contribute to improvements. The methodological issues are discussed in more detail in this Chapter, while the proposed approaches are applied in a number of case studies in the remainder of this dissertation. 
Paradigm Conflict: Econometric/Optimization versus System Dynamics (Section 32)

The optimization approach adapted by the economists aims at deriving strategis to solve the climate change problem in a cost-efficient manner. In order to apty available tools in a sound way, reduced and strongly parameterized models are usd which only include the most important state variables. The process-oriented systen dynamics approach aims at describing the global climate system, in order to analye the consequences of human activities. By trying to capture the main dynamc interactions within this system, those models become rather complex. The models ae meant to have an interpretative and instructive value, rather than being predictive.

Although both approaches are potentially able to support policy-making ina useful way, both approaches have limitations in capturing the whole range if scientific knowledge to support policy-making. My aim is to integrate the strengths of both approaches to derive a framework in which optimization oriented experimers are performed with advanced process-based simulation models.

\section{"Subjectuve" Uncertainty (Section 3.3)}

Global change is characterized by strong uncertainty and great irreversibility on the level of consequences, as well of probabilities of occurrence (Faucheux and Froger, 1995). The use of expected values is of limited value for these kind of problems, because such an approach ignores the notion that values, motivations, world views, and insights differ among the various agents. Furthermore, the use of expected values ignores that uncertainty about the functioning of the earth system and human society lies not only in statistical aspects and measurement errors, but also in fundamental uncertainties which can be made explicit by using the notion of different world views. Therefore, to include the notion of plurality of views on reality, a perspective-based uncertainty analysis is introduced.

\section{Structural Change (Section 3.4)}

The present integrated assessmient models are developed from a mechanistic perspective, where prediction and control are appropriate approaches. However, economic, social, technological, and biological systems cannot be adequately described by mechanistic modelling tools, nor can their structure and behavior be described only in terms of non-linear systems. For such systems, which show evolution and adaptation, we need new tools. These tools should include the learning, evolving and adapting processes which can be found in the new scientific stream of complex adaptive systems. An aim of this dissertation is to incorporate some elements of complex adaptive systems into integrated assessment modelling. 
In the next Sections, I will go into some more detail about the three methodological issues of integrated assessment modelling which are central in this dissertation.

\subsection{Optimization}

\subsubsection{Introduction}

Optimization refers to the act of achieving the optimum, the "best" solution. The output of an optimization model is a statement of the best way to accomplish a specific goal. Optimization models do not tell what will happen in a certain situation. They are normative models. Although many aspects of optimization theory have been known to mathematicians for centuries, the development of computer science made these methods attractive for practical application. Optimization and the more general Operations Research are widely used as input to operational decision-making, especially in industry. The optimization method requires that problems are formulated in a simple and unvarying format: maximize or minimize an objective function by controlling decision variables subjected to constraints.

When the problem has been stated in the proper format, it is solved by finding the right point(s) in the feasible area defined by the constraints and the objective function. The search process is mathematically extremely difficult and, therefore, the process is often simplified by linearization of the objective function and constraints. Also the lack of feedbacks and dynamics are often necessary simplifications to use suitable optimization tools.

Another limitation of optimization is the difficulty in specifying the objective functions in real life problems. The objective function embodies values and preferences, but which values and whose preferences? How can intangibies be incorporated into the objective function?

Thus, the normative approach gives problems in specifying the problem formulation, where the constraints of mathematics result in simplifications of the problem. However, the normative view of the world imposed by the optimization paradigm encourages discussion of concrete (policy) goals, which in itself might be a worthwhile exercise. In the next Section, I will discuss the use of optimization within integrated assessment modelling of global change. As an organizing framework, I will discuss the concept for the three steps ideally taken in controlling a system. 
Deciding how to control a system ideally involves three steps (Beightler et al, 1979)

\section{An accurate and quantitative description of the system.}

There is little point in optimizing a model that does not describe what is happening in the system; therefore, most efforts in practical optimization studies are directed to understanding the system and describing it in quantitative ways.

2. A single measure of the system effectiveness expressible in terms of the decision variables.

It is usually either trivially simple or practically impossible to accomplish a measure of the system effectiveness.

3. Find values of the decision variables so that the effectiveness of the system is optimized.

Given steps one and two, this step is "just" a mathematical exercise.

\subsubsection{Optimization Applied to Global (Climate) Change}

To date, various optimization oriented models for climate change exist, such as DICE, MERGE and CETA (see Chapter 2). The general aim of these models is to calculate under a range of assumptions optimal carbon tax levels (and eventually other decision variables), so that the discounted value of utility of consumption is maximized, with or without additional environmental constraints. I will discuss the three steps in optimization of Beightler et al. (1979) and analyze in which way these steps are taken, and discuss if some steps need to be reconsidered.

\section{1) Description of the system}

A typical limitation compared with the system dynamics approach is the limited description of the complex dynamics involved in the global system. Nordhaus (1993), argues that "existing [physical] models are, unfortunately, much too complex to be included in an economic model". However, the simplified climate system does not: likely deliver an adequate representation of the underlying systems' processes and dynamics. As a consequence, such an optimization model is too unrepresentative of what is known of the climate system (Price, 1995).

Of course, while a model is never a perfect representation of a problem related to global change, the optimal solution of a model is not an optimal solution of a problem. Two approaches are possible to this difficulty: simplifying the problem or relaxing the optimality constraints. Most of the current approaches follow the first, while I will analyze the prospects of the latter. My starting point is a description of the system, an integrated assessment model, which is within the limits of my approach an 
accepted representation of the state-of-the-art knowledge. Unfortunately, these kind of models are often large, complex non-linear systems to which traditional optimization tools can not be applied. As an alternative, however, heuristic search algorithms are able to find suitable (sub) optimal solutions (see step 3).

\section{2) Objective function}

If goals for future development can be formulated explicitly, they are not likely to be a single number. The concept of sustainable development, which is embraced by the majority of researchers and policy makers involved in global change, is not formulated in an explicit univocal way (Krause, 1995; Rothen, 1995). Optimization can, therefore, be seen as one of the many possible tools to analyze a problem, with a specific normative starting point, aimed at concrete policy questions.

\section{3) Solving the optimization problem}

Steps 1 and 2 are partly restricted by the mathematical tools available. Current optimization models for climate change use traditional optimization tools like the GAMS package (Brooke et al., 1988). The increasing use of nonlinear dynamics leads to a world which is fraught with discontinuities and noisy search spaces. Obviously, the traditional optimization techniques depending upon the restrictive requirements of continuity and derivative existence are unsuitable for all but a very limited problem domain. However, new (heuristic) optimization methods have been developed the last. decades, such as simulated annealing and genetic algorithms, which have successfully been applied on various problem domains (e.g. Kirkpatrick et al., 1983; Davis, 1987; Goldberg, 1989; Forrest, 1993). Therefore, it might be recommended to reconsider steps 1 and 2. I will apply in this dissertation an optimization approach on more sophisticated descriptions of the system.

\subsubsection{Applications in this Dissertation}

In this dissertation, I try to make some modest small, but firm steps in the process of solving the problems surrounding the optimization approach of integrated assessment modelling. The aim is to investigate whether or not I can use more acceptable descriptions of the system in an optimization framework. This means using simulation models which include important processes, feedbacks and interactions describing expected changes of the system, given an increased pressure of human activities. Applying heuristic search tools on complex simulation models lead to the dilemma of abandoning the nice mathematical conditions for optimality of simpler 
models. However, I try to explore the benefits without ignoring the mathematical consequences.

In Chapter 4, I will use the model OMEGA, which is a combination of DICE and IMAGE 1.0, to analyze in what strength optimal response strategies are dependent of the modelling approach used for the climate system. Furthermore, I will introduce a new optimization approach for large scale dynamic systems and compare its performance with several other optimization approaches.

OMEGA was a nicely formulated mathematical system; however, the simulation model TARGETS does not meet the strict requirements for traditional optimization tools like continuous derivatives. Therefore, the robust genetic algorithms are applied in Chapter 6 in the search for suitable energy policies by using the TARGETS model.

\subsection{Perspectives}

\subsubsection{Uncertainty in Perspective}

Scanning for suitable images of the future involves an explicit treatment of uncertainty. Vercelli (1994) distinguishes various modalities of uncertainty: situations of certainty exist when a unique, wholly reliable probability distribution reduces to just one value. Weak uncertainty is defined by a unique probability distribution, additive and fully reliable. Strong uncertainty is described by a distribution of nonadditive probabilities and/or by a plurality of probability distributions which are not fully reliable. Near ignorance exists when none of the conceivable probability distributions is reliable.

Global change is characterized by strong uncertainty and. large irreversibility on the level of consequences, as well as that of probabilities of occurrence (Faucheux and Froger, 1995). Next to incomplete information and understanding of the system, subjective judgment and disagreement originating from different interpretations of the blind spots can explain uncertainties in images of the future. I propose to capture uncertainties arising from subjective judgment and disagreement by the inclusion of various perspectives.

The attitudes towards society and the environment evolved during history are, to an important extent, determined by perspectives which have been classified in various ways. In recent years, there has been increasing recognition of the usefulness of various socio-cultural perspectives within the context of sustainable development, albeit in qualitative terms mainly (Schwartz and Thompson, 1990; Thompson et al., 1990; Rayner, 1991; Zweers, 1984; Riebsame, 1990; De Vries, 1989; Jastrow et al., 
1990; Colby, 1991; Coward and Hurka, 1993; Rörsch and De Hart 1993; Dotto, 1994; Rotmans et al., 1994;, WRR 1994).

A contribution which gives a general description of perspectives on natural and human systems and social relations has been made by Thompson ef al. (1990) in their Cultural Theory. I will use this theory in applying the notion of various perspectives. This theory is not chosen as being the "best" available concept, but for practical reasons. I tried to apply a theory which is thought to be suitable for my purposes, that is using it within (mathematical) models. I acknowledge that the theory has its limitations, as any theory has, but this makes it possible to make a first step in applying the plurality of norms and values within models.

\subsubsection{Cultural Theory}

Thompson et al. (1990) elaborate the concept of perspectives by introducing the notions cultural bias (shared values and beliefs) and social relations (pattern of interpersonal relations). By reference to these notions, a cultural perspective is defined as a more or less viable combination of social relations and cultural bias. The degree of viability of a perspective depends upon a mutually supportive relationship between a particular cultural bias and a particular pattern of social relations. Thompson et al, (1990) claim that five, and only five, perspectives - the hierarchist, egalitarian, fatalist, individualist, and the hermit - are sufficiently viable. The adoption to perspectives is a dynamic process. Change occurs because of "surprise", i.e. the discrepancy between the expected and the actual, which is of central importance in dislodging individuals from a previously adopted perspective. Adherents to each of the five perspectives are, as it were, in competition for new adherents to their particular perspective, but are dependent on one another at the same time. In other words, all of the perspectives are needed to ensure each one's viability (Thompson et al., 1990). For example, fatalists are necessary to hierarchists, since they constitute elements which can be managed, the existence of fatalists as consuming participants in markets is essential to individualists, while the existence of both hierarchists and individualists is essential to the egalitarians to provide the institutions which act as yardsticks for egalitarianism. Following Rayner (1991) the "active perspectives", i.e. the hierarchist, the egalitarian, and the individualist, are taken into account, so that the autonomous and the fatalist perspectives are disregarded. The hermit, as exponent of the autonomous perspective, is not interested in what happens in the world, but prefers to distance himself from it. The fatalist, for whom everything is a lottery (both humans and nature being unpredictable) is likewise not examined as such. Nevertheless, the fatalist plays a significant role in the overall system. Note that look at decision-makers within 
(international) organizations, assuming that such agents want to be a player in he game. If I would model personal decisions, the "powerless" agents are involvd, representing also the fatalistic world-view.

Rayner (1991) and Van Asselt and Rotmans (1996) argue that the actve perspectives are the extremes of a continuum which is used to describe all possile points of the view (Figure 3.1). I will now briefly characterize the active perspecties so that it enables me to operationalize aspects of Cultural Theory within the integraed assessment modelling activities.

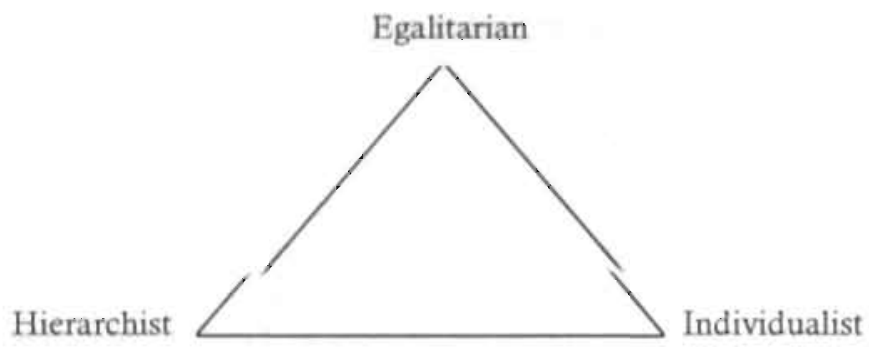

Figure 3.1 Spectrum expanded by the active perspectives.

\section{Grid-group Typology}

Douglas and Wildavsky (1982) proposed a grid-group typology so that societies can be characterized along two axes, labelled group and grid. The term group refers to the extent to which an individual is incorporated into bounded units. The greater the degree of incorporation, the greater the extent to which individual choice is subject to group determination. The term grid denotes the degree to which an individual's life is circumscribed by externally-imposed prescriptions. The more binding the prescriptions are, and the more extensive their scope, the fewer the facts of social life that are open to individual negotiations.

Along the grid dimension, it is the element of social control that sets the various perspectives apart from each other. Strong group boundaries coupled with minimal prescriptions produce strong relations that are egalitarian. Because such groups lack internal role differentiation, no individual is granted the authority to exercise control over another. When an individual's social environment is characterized by strong group boundaries and binding prescriptions, the resulting social relations are hierarchical. Individuals in this social context are subject to the control exerted by other members in the group, as well as to the demands of socially-imposed roles. The exercise of control (and more generally the very existence of inequality) is justified on 
the grounds that different roles for different people enable people to live together more harmoniously than alternative arrangements would. Individuals who are neither bound by group incorporation nor by prescribed roles inhabit an individualistic social context. In such an environment, all boundaries are provisional and subject to negotiation. Although the individualist is relatively free from control of others, this does not imply abstention from exerting control over others; the individualists' success is often measured in terms of the size of the following the person can command. The fatalist belongs to the category weak group boundaries coupled with binding prescriptions, but will not be discussed here (see Thompson et al., 1990).

\section{Views of Physical Nature}

Thompson et al. (1990) claim that ideas of human and physical nature are socially constructed and adopted by individuals according to their perspective. The concept of "myths of nature", derived from the work of ecologists (such as Holling (1977); Timmerman (1986)), may enable the cultural perspectives to be represented graphically by reference to the metaphor of a sphere rolling in a curved landscape (see Figure 3.2). Nature, for egalitarians, is strictly accountable, and their ideas of the world can be represented by the concept of "Nature Ephemeral" (Holling, 1977; Timmerman, 1986). For them, the world is a terrifying, unforgiving place and the least jolt may trigger complete collapse. The managing institution must therefore take good care of the ecosystem; the egalitarians advocate a preventive management style (Rayner, 1991). For individualists, nature is a "skill-controlled cornucopia" (Thompson et al, 1990), i.e. thanks to human skill, nature provides resources in plenty. The individualists' idea of the world is represented by the concept "Nature Benign" (Holling, 1977; Timmerman, 1986). According to this myth, the world is wonderfully forgiving. The management institution can, therefore, adopt a laissezfaire attitude, that is, the individualists advocate an adaptive management style (Rayner, 1991). The hierarchists see nature as being in an "isomorphic" (Thompson et al, 1990) relation with the social realm; i.e. its rich differentiation ensuring that it is forthcoming when approached in the right way by the right people, but retributive when pushed beyond these carefully-learned. bounds. This idea is represented by the myth of Nature as "Perverse/Tolerant" (Holling, 1977; Timmerman, 1986); Nature is forgiving in most circumstances, but is vulnerable to the ball flying over the rim on occasion. The managing institution must, therefore, take steps to prevent such unusual occurrences. The hierarchists, therefore, advocate the exercise of control as a management style. 


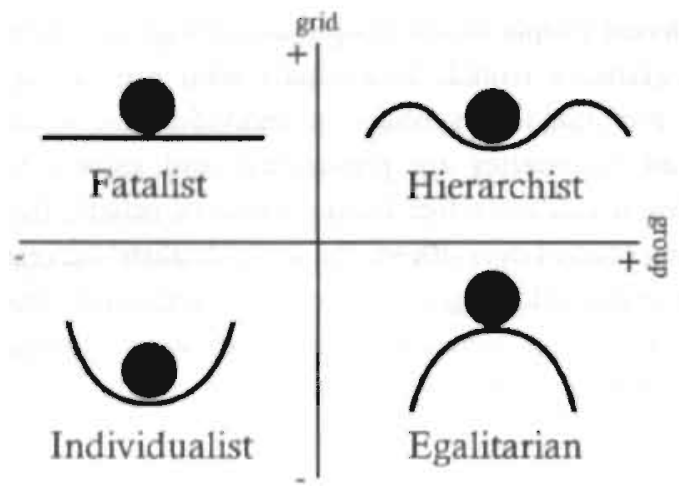

Figure 3.2: Cultural perspectives (Source: Schwartz and Thompson, 1990)

\section{Views of Human Nature}

According to Thompson et al. (1990), views of human nature are inextricably bound up with social relations. Egalitarians believe that human beings are born good, but are corrupted by evil institutions. From an egalitarian perspective, human nature is not only good, but also highly malleable. Just as human nature can be corrupted by evil institutions (e.g. markets and hierarchies), so can it be: rendered virtuous by constructing a noncoercive, egalitarian society. The optimistic view of human nature is essential to the viability of egalitarian social relations. For individualists, human nature, like physical nature, is extraordinary stable. No matter what the institutional setting, human beings remain essentially the same, i.e. self-seeking. By regarding man as self-seeking and unmalleable, individualists can justify attempts to channel existing human nature, and their refusal to attempt to change it. Hierarchists believe that: human beings are born sinful, but can nevertheless be redeemed by virtuous institutions. This conception of human nature helps sustain a perspective rich in institutional restraints.

\section{Strategies}

The manner in which people make a living is central to their lives. Thompson et al. (1990) argue that needs and resources are socially-constructed, so that the constraints on behavior are located in the perspectives, rather than in the needs and resources themselves. This leads to the conclusion that behavior is never rational or irrational in itself; a particular strategy can only be evaluated in relation to a person's perspective. Strategies do what is most important; i.e. they uphold a person's perspective. Because the egalitarians perceive resources as being fixed and believe that people can do nothing about them, the only available strategy is to decrease their 
needs so as to ensure a non-negative overlap. To be effective, such a need-reducing strategy would have to be followed by everyone. In perceiving nature as cornucopian, the individualists clearly have the scope to manage both needs and resources. Therefore, they choose to manage both needs and resources upward to the very limits of their skills. The hierarchists cannot do anything about their needs, so the only available strategy is to increase their resources so as to make sure that the overlap does not become negative. This approach is legitimized by a view of nature as bountiful, but within accountable limits. For each of the perspectives, preferences regarding topics such as economic growth or risks can be derived in a similar manner.

\subsubsection{Applications in this Dissertation}

During, the last few years, aspects of the Cultural Theory are used as a heuristic framework, to apply the notion of perspectives in integrated assessment models. A perspective-based uncertainty analysis is introduced as an approach to include plurality of world-views of policy agents (Janssen and Rotmans, 1995). We used cultural perspectives determined distributions of parameters and future scenarios in order to allocate emission rights of $\mathrm{CO}_{2}$. A detailed discussion of this study can be found in Chapter 7.

The successful introduction of Janssen and Rotmans (1995) of a quantitative approach to take into account subjective judgments and plurality of views on reality is followed by a more general approach for perspective-based uncertainty analysis, described in Van Asselt and Rotmans (1996). They worked out their alternative model routes for the fertility issue and the climate change issue. Currently, alternative model routes are being developed for all submodules of TARGETS and the model as a whole (Rotmans and De Vries, 1996).

The idea of "perspectives" is used to involve the different perceptual screens through which people interpret or make sense of the world and the social dimensions within it, whereby particular visions of reality seem more or less plausible. Trisoglio et al. (1994) characterize the cultural perspectives by two dimensions following Thompson et al. (1990) (Table 3.1):

1) how the world is seen (the totality of views of physical and human nature).

2) management style (the totality of preferred strategies).

The combination. of each perspective's world view with its respective favored strategy is used to assess the utopias (Trisoglio et al., 1994). Agents' utopia are used to describe the management style which corresponds with the agents' view of the world. The dystopias describe what would happen to the world if the agents' favored strategy were to be applied while reality seems to follow another world view. 
The utopia/dystopia approach as applied by Trisoglio et al. (1994), Van Asselt and Rotmans (1996) and Rotmans and De Vries (1996) is a static approach. Their approach can be seen as an agents' perspective of the future today, which determine their present policy which might consist of a set of decision rules. In their static approach, the agents do not react to observed dystopias. A first exercise to develop a dynamic version in which agents may adapt their behavior in case of a dystopia will be discussed in Chapter 8 .

\begin{tabular}{|c|c|c|c|}
\hline & individualist & hierarchist & egalitarian \\
\hline $\begin{array}{l}\text { world view } \\
\text { idea of nature }\end{array}$ & $\begin{array}{l}\text { skill-controlled } \\
\text { comucopia }\end{array}$ & isomorphic nature & accountable \\
\hline myth of nature & natural benign & $\begin{array}{l}\text { nature } \\
\text { perverse/tolerant }\end{array}$ & nature ephemeral \\
\hline $\begin{array}{l}\text { concept of human } \\
\text { nature }\end{array}$ & self-seeking & sinful & born good, malleable \\
\hline $\begin{array}{l}\text { management style } \\
\text { driving force }\end{array}$ & growth & stability & equity and equality \\
\hline type of management & adaptive & control & preventive \\
\hline attitude to nature & laissez-faire & regulatory & attentive \\
\hline $\begin{array}{l}\text { attitude towards } \\
\text { humans }\end{array}$ & $\begin{array}{l}\text { channel rather than } \\
\text { change }\end{array}$ & restrict behavior & $\begin{array}{l}\text { change social } \\
\text { environment }\end{array}$ \\
\hline $\begin{array}{l}\text { attitude to } \\
\text { needs/resources }\end{array}$ & expand resource base & $\begin{array}{l}\text { rational allocation of } \\
\text { resources }\end{array}$ & $\begin{array}{l}\text { need-reducing } \\
\text { strategy }\end{array}$ \\
\hline economic growth & $\begin{array}{l}\text { preferred: aim to create } \\
\text { personal wealth }\end{array}$ & $\begin{array}{l}\text { preferred: aim to avoid } \\
\text { social collapse }\end{array}$ & not preferred \\
\hline nisk & nisk-seeking & risk-accepting & risk-aversive \\
\hline
\end{tabular}

Table 3.1: Characteristics of Cultural Perspectives. 


\subsection{The Evolutionary Modelling Approach}

\subsubsection{The Evolution of Modelling}

Newton's publication of his Principia in 1687 is generally seen as marking the birth of classical science, and this mechanistic, reductionistic, and equilibrium-based explanation of the world was to prove successful in stimulating physical science. During the so-called Machine Age, research was dominated by selecting what was to be understood, studying the working of the parts, and assembling the understanding of the parts into an understanding of the whole. This came naturally to men whose view of the universe inspired them to create machines to do their (physical) work, and a product of such efforts was the Industrial Revolution itself. Moreover, the success of the mechanistic paradigm led to the application of the mathematic tools of physical science to life sciences such as economics, social science, and biology. Although the success of classical science was challenged in the first half of this century by new theories in physical science (quantum mechanics and relativity theory), the reductionistic and deterministic view, nevertheless, remained the core of science.

By the second quarter of this century, formulation of a new world view was brought about, in part, by the growing preoccupation with systems, with their growing complexity and the increasing difficulty of managing them effectively. This focus on systems led to the realization that they constitute wholes which lose their essential properties when taken apart. In metaphoric terms, the systems approach follows a holistic view, instead of the reductionistic view of classical (Newtonian) science.

Nevertheless, the dominant world view was still ruled by deductive logic and mathematics: the rationalistic world view. A reversible world in which initial conditions determine a reversible trajectory is postulated so that once the initial state of the system has been determined, deductive logic can chart the past and predict the future trajectories. Whatever has changed can be exactly undone by another change, and. using exact prediction, the behavior of Nature can be precisely controlled. A widely used metaphor of systems remains that of the engineer who is controlling a machine (cf. Kwa, 1984; 1994). However, Von. Bertalanffy (1968) noted that while dynamic systems can be used for describing the maintenance of a system, they cannot explain change, diversity, and evolution, and, since as early as 1948, Warren Weaver (Weaver, 1948) has been stressing the need for an approach which deals with a sizable number of factors which are interrelated into an organic whole, pointing to biological, medical, economic, and social issues.

There has thus been a growing realization that if long-term processes in social and. biological systems are to be studied effectively, the previous approaches are of limited 
use, since societies, humans and other organisms live in continuous and changing interactions with their environment, leading to structural changes. Furthermore, since each biological or social agent has specific characteristics, the survival of the fittest means that individuals are not equally successfull. In metaphoric terms: there is a shift from viewing the system as a machine to the system as an organism.

\subsubsection{Complex Adaptive Systems}

The rapid development of the computer has provided scientists with a new scientific tool, so that during recent decades, various mathematicians, computer scientists, ecologists, etc. have been developing new tools and novel approaches to facilitate their efforts to investigate complexity in life sciences. Despite the disparities and the difficulties, with these new approaches we are entering a new era in our ability to understand and foster biological and social systems (Holland, 1992b). Scientists have begun to detect a common kemel from these systems, each of which involves a similar "evolving structure" whereby they change and organize their components to adapt themselves to the problems posed by their surroundings. Such systems can be grouped under a common name: complex adaptive systems. In recent decades, new computation-based modelling tools have been developed which enable these systems to be studied, among which we find: genetic algorithms, cellular automata, and artificial life forms. Such evolutionary modelling techniques have been applied in various disciplines which study, for example: economies, ecologies, immune and nervous systems (e.g. Anderson et al., 1988; Goldberg, 1989; Langton, 1989; Arthur, 1990; Kauffman, 1991; Holland and Miller, 1991; Holland, 1992a; Waldrop, 1992; Ruthen, 1993; Epstein and Axtell, 1995; Trisoglio, 1995). Although this new modelling paradigm is likely to derive new insights from various complex systems, it still has to prove itself (Horgan, 1995).

In order to highlight differences between the "Newtonian" approach, the systems approach, and the complex adaptive systems approach, I propose to discuss some of their respective characteristics (Table 3.2). The Newtonian approach offered us universal laws, the systems approach a holistic general cause-effect diagram of the system, while complex adaptive systems deal with the diversity of individual characteristics of the agents.

If the Newtonian approach could describe how systems move to an equilibrium, and complex systems the pathways between multi-equilibria, complex adaptive systems, in contrast, can be seen as evolving over time, thereby adapting to the continuous changes within the system. 
The mechanistic perspective of Newtonian science and the systems approach allow us to construct models in order to optimally control the system and to predict future developments. According to the complex adaptive systems approach, however, changes in structure and behavior can neither be predicted nor fully controlled.

The information exchange between the various elements in the system differ between the approaches. The Newtonian approach assumes perfect knowledge leading to an equilibrium state of the system. In the (complex) systems approach, the information about the state of various parts of the system is modelled through feedback mechanisms. In the complex adaptive systems approach, the agents have preferences and expectations leading to anticipations of their behavior. Differences in expectations and realized behavior of the system lead to adaptive behavior or physical adaptation of the agents.

The complex adaptive systems approach requires a novel (game-oriented) way of dealing with models, a problem which is currently underestimated. An approach of using models will be required which accommodates the notion that the future is not only uncertain, but also inherently unpredictable. This may yield insights, but no precise answers as was claimed by Newtonian science.

Table 3.2 Characteristics of three modelling paradigms.

\begin{tabular}{|l|l|l|}
\hline \multicolumn{1}{|c|}{ Newtonian Science } & (Complex) Systems & Complex Adaptive Systems \\
\hline reductionist & holistic & holistic \\
answers & insights & insights \\
mulibrium & multi-equilibria & no equilibrium \\
top-down & top-down & bottorm-up \\
homogeneity & homogeneity & heterogeneity \\
mechanistic. & mechanistic & organic \\
prediction and control & control & $\begin{array}{l}\text { unpredictability and } \\
\text { (un)controllability }\end{array}$ \\
perfect knowledge & feedback-control & adaptation / anticipation \\
reversibility & reversibility & irreversibility \\
structural constancy & structural constancy & evolution/structural change \\
\hline
\end{tabular}




\subsubsection{An Evolutionary Integrated Modelling Approach}

The present generation of integrated assessment models is mainly based on the mechanical paradigm. The system is thought of as a machine. Mechanical causality, expressing overarching natural laws explains all things. The increasing pressure of human activities causes the environmental system to be forced out of the preindustrial "natural" equilibrium. This disequilibrium is thought to be a threat which needs to be solved by reducing the pressure to a level such that the system finds a new equilibrium again. According to Allan (1990), equilibrium models are a most unlikely basis for anticipating the future, particularly with respect to change. Instead of discussing idealized equilibrium systems and focusing on stability, we must try to understand instability and change. Mechanical systems are only capable of "functioning", not of evolving. They are unable to restructure themselves or to insert new relationships, while, in reality, this can happen.

Scanning the future of the global system for the next century, without considering the ability of systems to adapt to changes, may generate a misleading picture of the impact of changes. Ecosystems may adapt to a changing climate, human behavior to changing economies and insects to the use of insecticides. From an anthropocentric view, risks occur when changes are so fast that systems are not able to adapt. A reduction of the diversity within the system makes it more fragile and less capable of adaptation. From a mechanistic perspective, risks may increase if the system changes (= out of equilibrium), while, from an evolutionary perspective, risks may increase if the system is less able to adapt to a change.

The inclusion of evolutionary aspects within integrated assessment models may be important to assess the impacts of present and future human activities on the global system. As noted before, integrated assessment modelling, is a relatively young discipiine, where various approaches are currently applied. The future will tell us which species of modelling is the fittest in the policy-making process. It is my expectation that an evolutionary modelling approach will be a powerful alternative to the existing ones.

\section{Change}

An interesting framework to explain the transitions in behavior of the system is the approach of Holling (1986), who proposes four basic functions common to all. complex systems and a spiralling evolutionary path through them (Figure 3.3). This evolutionary cycle can be used to explain transitions in social systems, as well as in ecosystems, which is done by Holling et al. (1995). The central idea is that the four phase adaptive cycle emphasizes a loop from conservation to two phases of 52 
destruction and reorganization where innovation and chance assume a dominant role. The reorganization phase occurs when a rare and unexpected intervention or event can shape a new future. Holling et al. (1995) argue that in this stage, when the system is in a state most likely to be transformed by innovation, agents have the greatest potential to influence the future of the system. Note that the system can also exit the cycles and flip into a new kind of system.

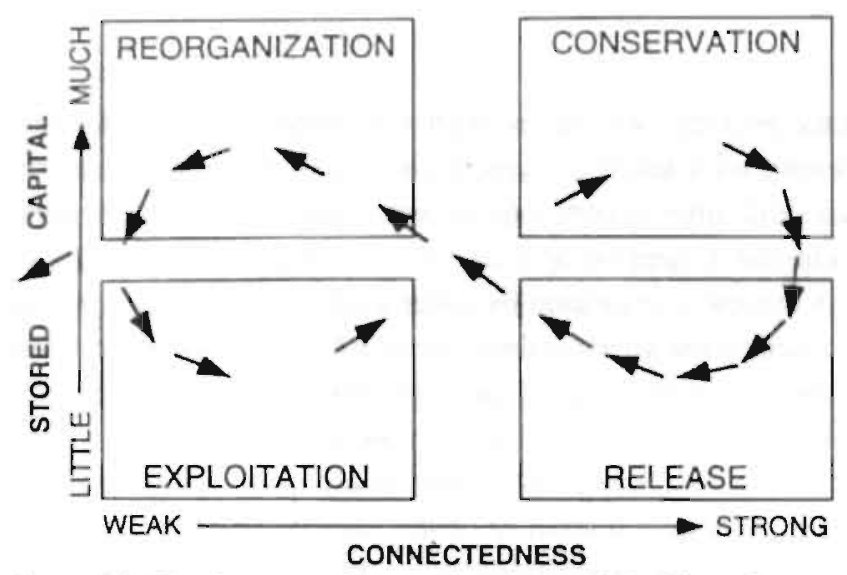

Figure 3.3: The four general system functions and the flow of events between them from Holling, 1986). The arrows show the speed of that flow in the ecosystem cycle; arrows close to each other. indicate a rapidly changing situation and arrows far from each other indicate a slowly changing situation. The cycle reflects changes in two attributes: on the $Y$ axis, the ameunt of accumulated capital (nutrients and carbon) stored in variables that are dominant keystone variables at the moment, and on the $\mathrm{X}$ axis, the degree of connectedness among variables. The exit from the cycle indicated at the left of the figure indicates the stage where a flip is most likely into a less- or more-productive and organized system, that is, devolution or evolution as revolution.

The patterns produced by these four phases are discontinuous and dependent on the interaction of processes that trigger and organize the release and reorganization functions. These interactions create multiple stable states. An illustrative example is the budworm outbreak (Holling, 1978). Successful suppression of spruce budworm populations in eastern Canada using insecticides certainly preserved the pulp and paper industry and employment in the short term by partially protecting the forest. However, this policy has left the forest and the economy more vulnerable to an outbreak over a larger area and of an intensity not experienced before. This example illustrates that problems could be caused by local human influences that slowly accumulate to trigger sudden abrupt changes that may effect the vitality of societies. There are counteractive forces that give ecological systems the resilience and 
adaptability to deal with considerable change and that provide people with the capacity to innovate and create (Holling, 1994). However, nature, people, and economies are suddenly now co-evolving on a planetary scale, each affecting the others in such novel ways and on such a large scale that large surprises may overwhelm the adaptive and innovative capabilities of people (Holling, 1994). The question of sustainable development is, therefore, how to stimulate a sustainable coevolution of human activities and environmental change.

\section{Evolution}

Early-nineteenth-century biology was faced with the concept of unchanging species. Just like Newton's laws were taken as signs of God's law immanent on earth, the diversity of species and similarities between them, were taken to be God's work. Publication of Darwin's On the Origin of Species (1859) changed the insights of biological science. Darwin's theory of evolution by natural selection - the survival of the fittest - fundamentally changed the view on the place of Man within the evolution of life. Without further discussing the impact of Darwin's theory, the scientific understanding of the evolutionary process has grown enormously. Was Darwin's theory controversial one century ago, nowadays evolutionary principles are widely accepted due to, for example, Mendel's discovery of the basic laws of transmission genetics, Weismann's concept of the continuous germ plasm from which each organism grows, and the advent of population genetics in the effort to account for Darwinian evolution by selection acting on single genes (Kauffman, 1993).

Regarding global change, we are interested in changes on Earth in the coming century. Is a theory, which explains the evolution of life during the past several billion years, of use for only a snapshot of evolutionary time? For this very short time horizon evolutionary principles are only of use for species which genetic evolution is rather fast. The resistance development of insects to various pesticides during this century is an example of genetic evolution within the considered time-horizon. Next to genetic evolution, changes in behavior can also be explained in evolutionary terms. With respect to human beings, this could be called a cultural evolution. Because changes of behavior are not likely to depend on the exchange of genetic information, its timehorizon is much shorter than genetic evolution. In the next paragraphs, I will discuss briefly the two types of evolution.

\section{Genetic Evolution}

Most higher organisms evolve by means of two primary processes: natural selection and sexual reproduction. The first process determines which members of a population survive to reproduce, and the second process ensures mixing and 
recombination among the genes of their offspring. An obvious biological consequence of sexual reproduction is the generation of new combinations by mixing genetic information from different individuals. Without this mixing generated by sex, adaptive evolution would simply consists of the sequential selection of mutations in the genetic information. Selection takes place on the basis of the fitness of the organisms, which is related to the probability of getting descendants (Hofbauer and Sigmund, 1988).

\section{Cultural Evolution}

The diverse individual experimentations which, when successful, can be imitated by others, where the storage medium for passing on behavioral norms consist of, for example, oral tradition, books and films. The fitness functions measuring the "success" is in the "mind" of each individual concerned, and here selection will depend on diverse systems of norms and values, leading to cultural evolution (Allen, 1990). Such an evolution is faster than genetic evolution, which requires the physical elimination of the "unfit". But the discovery of better strategies, at least in the shorter term, and the concealment or diffusion of this information to other individuals become the key elements in cultural evolution.

The insights derived from the evolutionary paradigm is used to construct a conceptual framework which is aimed at serving as an integrated modelling paradigm for global change.

\subsubsection{An Integrated Modelling Paradigm}

Research with respect to sustainable development often integrates disciplines from social science, economics, and ecology. Although many attempts are made to come to an integrated approach, it is my opinion that they all fail by considering only one disciplinary modelling paradigm. When modelling the global system, various levels of modelling may be distinguished. De Vries (1994), for example, distinguishes three levels. At the first level, he assumes the physical stocks and flows which constitute the observable reality. The next level maps the behavioral and informational structures which govern human interference in the underlying physical environment. The third level are the values, beliefs, and ideas which here are shared under the term perspectives, and those reflect and motivate people's behavior.

Based on De Vries (1994), three interwoven levels are distinguished in constructing an integrated modelling paradigrn for global change (Figure 3.4). At the first level, the physical laws are assumed to work. The flows of minerals through the various reservoirs can be described satisfactorily by sets of differential equations, as 
can with topics as atmospheric chemistry, hydrology, and thermodynamics. In the objective to study global change, a time horizon of about one century may be considered. Given this assumption, I believe it is permitted to assume that the mechanisms and structure of the physical system remain unchanged. Of course, the flows between the reservoirs may change due to living and non-living part of the Earth system, but to describe this part of the system, a deterministic description is likely to be satisfactory.

At the second level, I assume is the living part of the Earth system. In this part of the global system, change in its structure and mechanisms occurs frequently within the considered horizon. Three fields are considered within this part of the system: economy, describing the interactions between agents who maximize their utility by exchanging resources "labor, capital, and the environment; sociology, describing the behavior of individuals in groups and behavior between groups; ecology, describing the behavior of organisms in relation to their environment. The many interacting agents lead to unpredictable behavior at the macro scale; I assume that agents have no perfect knowledge and do not act purely rationally. Such a system might well be described by the concepts of complex adaptive systems. At the third level, I present the driving forces to the behavior of agents: their needs, norms and values. I propose not to describe this level in mathematical formulas, although describing the behavioral "rules" at the second level might be clarified by conceptual models of the third level. Using the Cultural Theory in describing different management styles and world views is an example of the use of such a conceptual model.

In sum, the integrated modelling paradigm consists of the following elements. At the level of physics, a mechanistic approach is assumed where a set of deterministic differential equation determine the behavior of the physical elements. The levels biology, economy, and sociology are dominated by complex adaptive systems, like genetic algorithms and cellular automata. At the level of philosophy, conceptual models like Cultural Theory might be suitable to describe the agents' behavioral rules. 


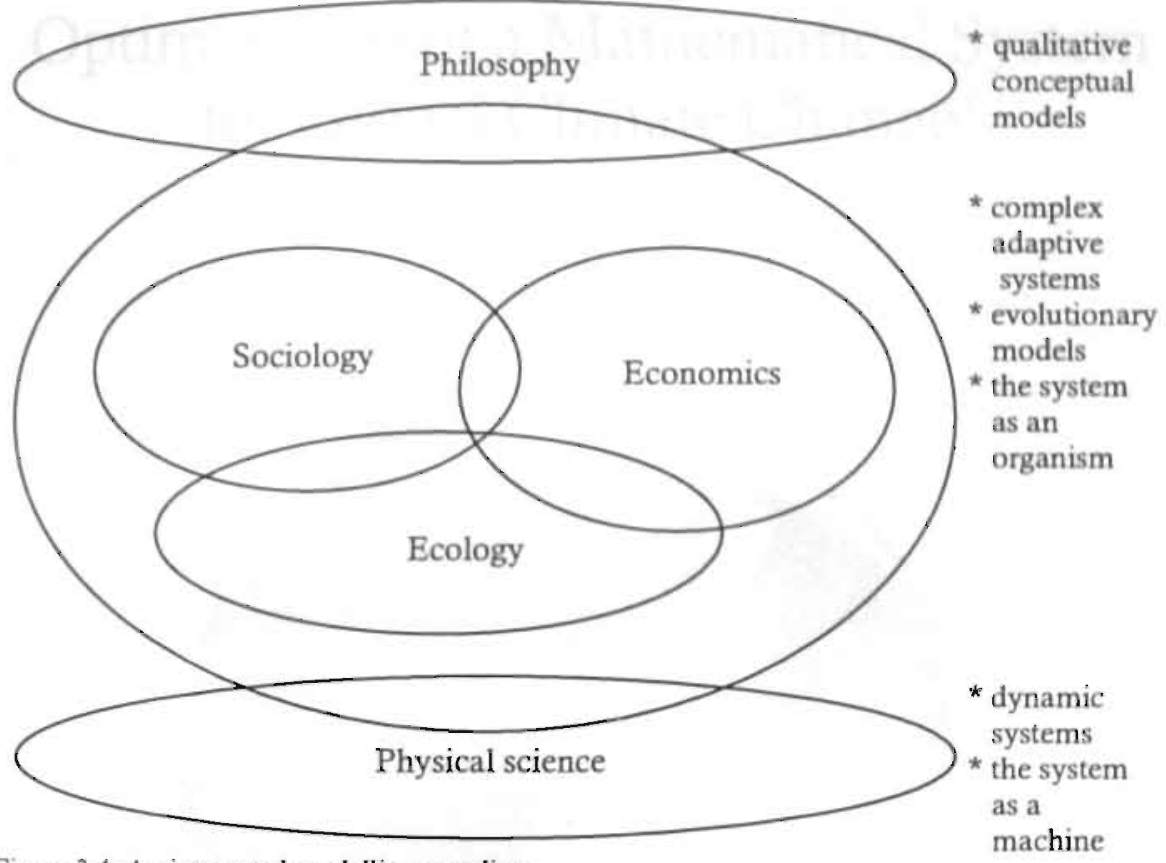

Figure 3.4: An integrated modelling paradigm.

\subsubsection{Applications in this Dissertation}

Some first sketches are made in the development of an evolutionary modelling approach of integrated assessment modelling. Instead of aiming at a complete integrated assessment model along the lines of evolutionary modelling. I performed two case studies in assessing change and surprise in social and biological systems. The first case study (Chapter 8) deals with the ability of agents to change their behavior, if their perspectives on the considered problem are changed. A set of agents are modelled having different positions within the perspective triangle (Figure 3.1). Comparison of observations with expectations may lead to a reconsideration of the problem, inducing a possible shift in the world view of the agents. This concept of "the Battle of Perspectives" is an. example of how to integrate the various modelling paradigms for the types of dynamics. The second case study (Chapter 9), which analyzes the malaria problem, examines the ecological part of the system, namely the adaptation of mosquitoes and parasites to climate change, insecticides, and antimalarial drugs. 



\section{4 \\ Optimization of a Mathematical System for Global Climate Change ${ }^{1}$}

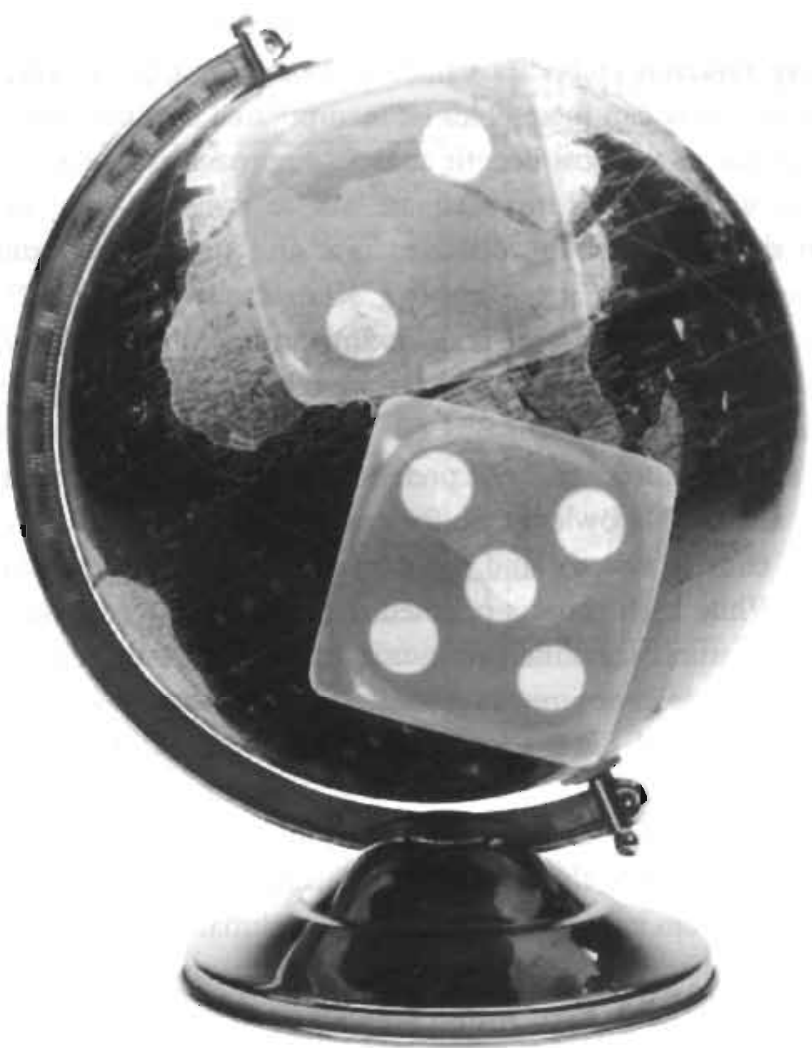

'This Chapter is based on Janssen et al. (1995), Filar et al. (1996), Janssen (1996a; 1996b), Janssen and Vrieze (1996). 


\subsection{Introduction}

An important stream in the integrated assessment modelling of climate change is the optimization approach as used in economics. As discussed in Chapter 2 and 3 , this differs from the simulation approach by the fact that it uses mainly simplified regression-based relations instead of process oriented system dynamic models, in order to apply traditional optimization techniques.

The economic type of modelling usually optimizes response policies to balance the costs and benefits; one can identify two kinds of limitations to the use of optimization. Firstly, the objective functions are difficult to specify for climate policy (see Chapter 3). The objective function embodies values and preferences, but which values and whose preferences? How can intangibles be incorporated into the objective function? For example, insofar as the cost benefit analysis approach is concerned, there is no consensus about whether it is an adequate aid to policy-making because of the arbitrariness in the choice of the discount rate and the inherent uncertainties in monetizing the impacts of climate change (e.g. Krause et al., 1995; Costanza, 1996).

Secondly, in order to render large-scale optimization problems tractable, modellers are obliged to simplify their systems, which they achieve by means of linearization of the system and exclusion of numerous feedbacks and dynamics (Sterman, 1991). Although such simplifications might be preferable from a mathematical point of view, they imply that scientific knowledge of, for example, the non-linear process-oriented dynamics of the climate system, and the existence of many important feedbacks is simply ignored. While such process-oriented dynamics and feedbacks are on top of the agenda in the natural scientific community, they are, nevertheless, neglected for mathematical arguments in optimization studies. Thus, although claiming performing integrated assessment modelling, a lot of economic oriented models do not include state-of-the-art knowledge.

In this Chapter, I will discuss the latter limitation of optimization. More specifically, I will investigate the limitations of using very simplified descriptions of the environmental system in optimization studies of climate change. I will address the question about whether or not an optimal response strategy is sensitive to the modelling approach which is used within an integrated assessment model. This is to analyze the need for incorporating more advanced modelling frameworks within optimization studies for global change, in general, and climate change, in particular. In the analysis, I will focus on the climate system part of the model.

In this Chapter, I employ a mathematical system for climate change OMEGA (Optimization Model for Economic and Greenhouse Assessment) (Figure 4.1), which combines DICE (Dynamic Integrated model of Climate and the Economy) 60 
(Nordhaus, 1992; 1993, 1994) and IMAGE 1.0 (Integrated Model to Assess the Greenhouse Effect) (Rotmans, 1990), from which the dynamic system representation of the environmental system is borrowed, (Braddock et al., 1994; Zapert, 1994), allowing me to use a set of non-linear optimization techniques. DICE is primarily an economic model enlarged by the addition of an oversimplified environmental model, while IMAGE 1.0 describes the climate dynamics from a natural scientific perspective. By using the economic component of DICE and the biophysical system component of IMAGE 1.0, a hybrid model is obtained in which the strengths of each model are used to overcome the weaknesses of the other.

\section{Human System Environmental System}

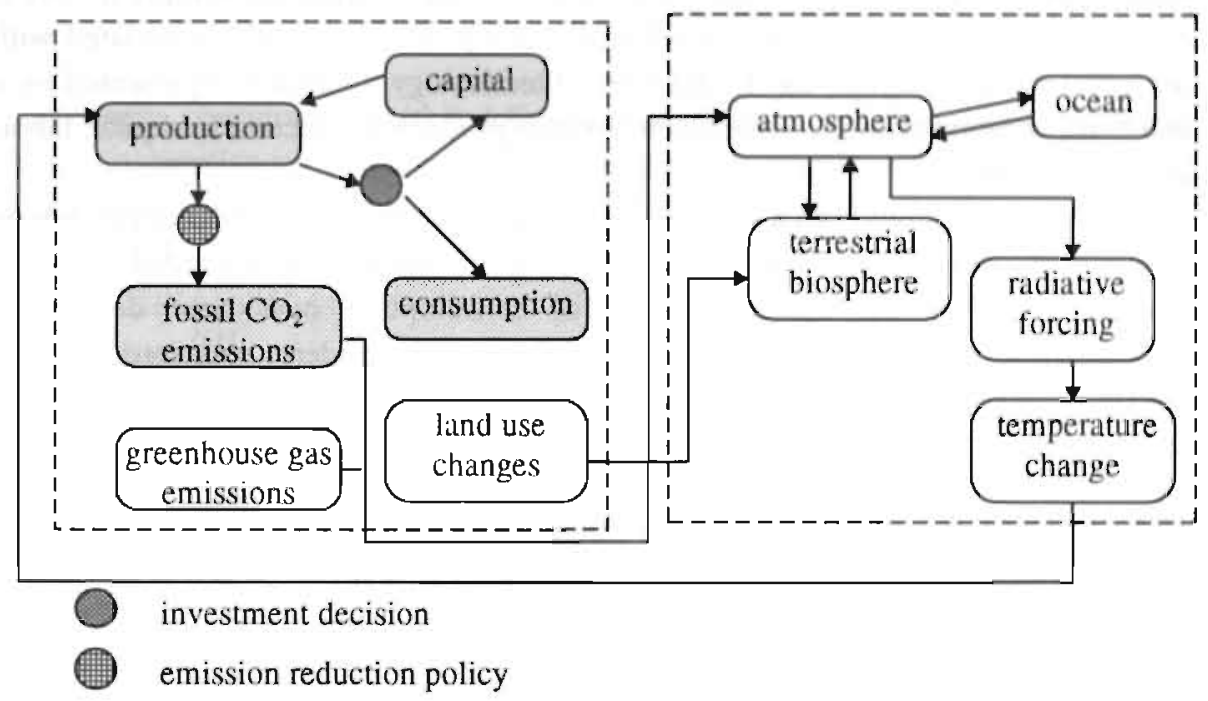

Figure 4.1: Scheme of the basic relations within the OMEGA model. The grey blocks come from DICE; the white blocks from IMAGE 1.0 .

In addition, in this Chapter, I will introduce a new optimization approach, sequential reduced-system programming, which is able to derive optimal solutions for large systems in a limited amount of time. The performance of this approach is compared with three existing approaches: the penalty method, sequential quadratic programming, and genetic algorithms. Such a comparative analysis is also made, because the limitations of optimization algorithms are generally used as argument for using very simple systems, and, furthermore, to get insights into the performance of 
genetic algorithms, which are used in Chapter 6 in an optimization study of the integrated assessment simulation model for global change TARGETS.

\subsection{The Model OMEGA}

\subsubsection{DICE: Dynamic Integrated Model of Climate and the Economy}

DICE is an optimization model for the economics of climate change based on aspects of optimal growth theory. It is a transposition of Ramsey's model (Ramsey, 1928) of optimal economic growth to climate change policy. Hence, as well as calculating optimal capital accumulation, DICE calculates greenhouse gas emission reduction by maximizing the discounted value of utility from consumption. DICE envisages the world economy as producing a composite commodity associated with an initial stock of capital, labor, and level of technology. Output is represented by a standard, constant-return-to-scale Cobb-Douglas production function in capital, labor, and technology.

The model contains both a conventional economic component and a novel climate component. Population growth and technological changes are regarded as being exogenous, while the optimized flow of consumption over time determines accumulation of capital. The climate component considers emissions of two important greenhouse gases. Carbon dioxide $\left(\mathrm{CO}_{2}\right)$ and $\mathrm{CFCs}$ are related to gross output, exogenous technological advances and an emission reduction factor.

In the mathematical formulation of DICE, the state variables are represented by $y(t) \in R^{9}$ which are dependent on the control variables $z(t) \in R^{2}$.

\section{Dbjective Function}

The DICE model is designed to model a situation in which the discounted sum of the general level of consumption achieves a maximum. The objective function maximized is:

$$
\max _{z_{1}(t), z_{2}(t)} \sum_{t=19000}^{2100} l(t) \cdot \ln \left(y_{2}(t)\right) \cdot(\tilde{l}+\rho)^{(19000 \cdot 0)}
$$

which expresses the discounted sum of utilities of consumption summed over the relevant time horizon. The level of utility or social well-being is expressed in $\ln \left(\mathrm{y}_{2}(\mathrm{t})\right)$ whereby $y_{2}(t)$ describes the level of consumption per capita at time $t, 1(t)$ is the size of population at time $t$, and $r$ is the pure rate of social time preference ( $r=3 \%$ per annum). 
The control variables $\mathrm{z}_{1}(\mathrm{t})$ and $\mathrm{z}_{2}(\mathrm{t})$ represent gross investment and the rate of emission reduction, respectively.

\section{Economics Component}

Output $\mathrm{y}_{1}(\mathrm{t})$ is given by a standard constant-returns-to-scale Cobb-Douglas production function in the levels of technology $a(t)$, capital $y_{3}(t)$ and labor which is assumed proportional to $\mathrm{l}(\mathrm{t})$, where labor inputs are proportional to population and $\gamma$ represents the elasticity of output with respect to capital taken as 0.25 . The impact of emission reductions and global climate change on output is represented by the scale factor $\mathrm{y}_{9}(\mathrm{t})$.

$$
y_{1}(t)=y_{9}(t) \cdot a(t) \cdot y_{3}(t)^{\gamma} \cdot l(t)^{L-\gamma}
$$

Per capita consumption $y_{2}(t)$ is defined as the ratio of consumption to population, where consumption is equal to economic output minus gross investments,

$$
y_{2}(t)=\frac{y_{1}(t)-z_{2}(t)}{l(t)}
$$

The capital balance equation for the capital stock $y_{3}(t)$ is,

$$
\frac{d y_{3}(t)}{d t}=z_{2}(t)-\delta_{k} \cdot y_{3}(t)
$$

where $\delta_{k}$ is the rate of depreciation of the capital stock, $10 \%$ per annum, reflecting an average lifetime of capital of ten years on a declining balance method.

The final economic equation represents the fossil and non-fossil emission amount of $\mathrm{CO}_{2}$ and $\mathrm{CFCs}$ related to economic output:

$$
y_{4}(t)=\left[1-z_{2}(t)\right] \cdot \sigma(t) \cdot y_{1}(t)
$$

where $\mathrm{z}_{2}(\mathrm{t})$ is the rate of emission reduction and $\sigma(\mathrm{t})$ the trend in $\mathrm{CO}_{2}$ equivalent emissions per unit of gross output in the absence of emission controls. 


\section{Environmental System}

The accumulation and transportation of the greenhouse gases in the atmosphere can be expressed as follows:

$$
\frac{d y_{s}(t)}{d t}=\beta \cdot y_{d}(t)-\delta_{m} \cdot\left[y_{s}(t)-y_{s}(1900)\right]
$$

where $y_{5}(t)$ is the concentration of $\mathrm{CO}_{2}$ in the atmosphere, $\mathrm{B}$ is the marginal atmospheric retention rate (the fraction of an emission that remains in the atmosphere in the short run) which equals $0.64 . \delta_{m}$, whereby the rate of transfer from the atmosphere to the oceans is $0.00833 \%$ of the excess of $\mathrm{CO}_{2}$ in the atmosphere per annum.

The relationship between greenhouse gas accumulation and increased radiative forcing, $y_{6}(t)$ in $W / m^{2}$ is given as:

$$
y_{6}(t)=4.1 \cdot\left\{\frac{\ln \left(y_{s}(t) / y_{s}(1990)\right.}{\ln (2.0)}\right\}+O(t)
$$

where $\mathrm{O}(\mathrm{t})$ represents other greenhouse gases such as methane $\mathrm{CH}_{4}$ and ritrous oxide $\mathrm{N}_{2} \mathrm{O}$.

The increase in globally averaged temperature in the atmosphere and the upper level of the ocean is expressed as:

$$
\frac{d y_{7}(t)}{d t}=\left\{1 / R_{1}\right) \cdot\left(y_{6}(t)-y_{7}(t) / \lambda-\left(R_{2} / \tau_{12}\right) \cdot\left[y_{7}(t)-y_{8}(t)\right]\right\}
$$

where $y_{8}(t)$ is the temperature increase in the deep oceans, $R_{1}$ and $R_{2}$ are the therrnal capacities of the upper layer and the deep ocean respectively, $\tau_{12}$ is the transfer rate from the upper layer to the lower layer, and $\lambda$ the climate sensitivity parameter $\left(\mathrm{W} / \mathrm{m}^{2 \circ} \mathrm{C}\right)$.

The temperature of the deep ocean is modelled as:

$$
\frac{d y_{8}(t)}{d t}=\left(1 / \tau_{12}\right) \cdot\left[y_{7}(t)-y_{8}(t)\right]
$$




\section{Impact on Economic Growth}

The scaling factor $y_{9}(t)$ is the ratio of 1 minus the percentage abatement cost and 1 plus the percentage of damage costs:

$$
y_{9}(t)=\frac{1-b_{1} \cdot z_{2}(t)^{b_{2}}}{1+\theta_{1} \cdot y_{7}(t)^{\theta_{2}}}
$$

The (market) damage costs are quantified as a relation between global temperature $y_{7}(t)$ increase and income loss, where $\theta_{1}$ represents the scale of damage and $\theta_{2}$ the nonlinearity in the damage function. The cost of reducing emissions of greenhouse gases are related to $z_{2}(t)$, the fractional reduction of greenhouse emissions, while $b_{1}$ and $b_{2}$ represent the scale and non-linearity of the cost function.

\subsubsection{IMAGE 1.0: The Integrated Model to Assess the Greenhouse Effect}

A mathematical system extracting the structure of IMAGE 1.0 (see Chapter 2) as a system of 155 differential equations has been developed to investigate the stability of the simulation. model (Braddock et al., 1994; Zapert, 1994); it is this system that is used in this study.

The mathematical representation of IMAGE 1.0 is of the form

$$
\begin{gathered}
\frac{\mathrm{d}}{\mathrm{dt}} \mathrm{x}(t)=F(\mathrm{x}(t))+\mathrm{u}(t) \\
\mathrm{x}\left(t_{0}\right)=\mathrm{x}_{0}
\end{gathered}
$$

where time $t \in[1990,2100], t_{0}=1990$ is the initial simulation time, $x(t) \in R^{155}$ is a vector of state variables, $u(t) \in R^{155}$ is the forcing term and $F(x(t)): R^{155} \rightarrow R^{155}$ describes the biophysical system. The solution $\mathbf{x}(\mathrm{t})$ as a function of time is a trajectory of equation (4.11). For a detailed description of the formulation of the mathematical system of IMAGE 1.0 I refer to Zapert (1994).

In representing the system components, the state vector $x=x(t) \in R^{155}$ at time $t$, is partitioned into the following variable groups.

$$
\mathrm{x}=\left[\mathrm{x}^{(1)}, x^{(2)}, \mathrm{x}^{(3)}, \mathrm{x}^{(4)}, \mathrm{x}^{(5)}, \mathrm{x}^{(6)}, x^{(7)}, \mathrm{x}^{(8)}\right]
$$

where 
$x^{(1)} \quad=\left(x_{1}, \ldots, x_{12}\right) \in R^{12}$, the carbon contents of the boxes in an ocean box diffusion model, in giga tons of carbon ( $\mathrm{GtC}$ ).

$\mathrm{x}^{(2)}=\left(\mathrm{x}_{13}\right) \in \mathrm{R}$, the concentration of carbon as carbon dioxide $\left(\mathrm{CO}_{2}\right)$ in the atmosphere, in parts per million by volume (ppmv).

$x^{(3)}=\left(x_{14}, \ldots, x_{62}\right) \in \mathrm{R}^{49}$, the carbon content in seven levels of the seven categorized ecosystems, in GtC.

$x^{(4)}=\left(x_{63}, \ldots, x_{69}\right) \in R^{7}$, the area of land in Africa, South America and South East Asia, allocated to each of seven ecosystems, in hectares.

$x^{(5)} \quad=\left(x_{70}, \ldots, x_{91}\right) \in R^{22}$, land use changes in Africa, South America and South East Asia.

$x^{(6)} \quad=\left(x_{92}, ., x_{140}\right) \in R^{49}$, the change in temperature since 1900 , in the 49 layers of the ocean under the mixed upper ocean layer, in degrees Celsius.

$x^{(7)}=\left(x_{141}\right) \in R$, the change in temperature since 1900 , in the mixed surface layer of the ocean, in degrees Celsius.

$x^{(i)}=\left(x_{142}, \ldots, x_{155}\right) \in R^{14}$, the concentration of various CFC gases with $x_{142}, \ldots x_{151}$, in pptv, carbon monoxide, $\left(x_{152}\right)$ and methane, $\left(x_{153}\right)$ in ppmv, and nurous oxiac, $\left(\mathrm{x}_{154}\right)$ in ppbv, in the atmosphere and $\left(\mathrm{x}_{155}\right)$ the production of $\mathrm{OH}$.

The forcing term $\mathbf{u}(t)$ is a 155 -vector partitioned according to the state vector partitioning and represents the human interference in the system (4.11). The non-zero components of $u(t)$ are associated with blocks $x^{(2)}, x^{(5)}$ and $x^{(8)}$. Therefore

$$
\mathrm{u}=\left[0, \mathrm{u}^{(2)}, 0,0, u^{(5)}, 0,0, u^{(8)}\right]
$$

where

$\mathrm{u}^{(2)}=\left(\mathrm{u}_{13}\right) \in \mathrm{R}$, emission of $\mathrm{CO}_{2}$ from fossil fuel combustion.

$\mathbf{u}^{(5)} \quad=\left(\mathrm{u}_{70}, . ., \mathrm{u}_{91}\right) \in \mathrm{R}^{22}$, ecosystem transfer rates, the amount of land per annum allocated from one ecosystem to another.

$\mathbf{u}^{(8)} \quad=\left(\mathrm{u}_{142}, \ldots, \mathrm{u}_{155}\right) \in \mathrm{R}^{14}$, other than carbon dioxide greenhouse gas emissions.

Implicitly, the forcing term $\mathrm{u}(\mathrm{t})$ includes the given scenarios estimation of future population growth, fossil fuel combustion, deforestation, and the technology development in the period 1990-2100 (Rotmans, 1990; Braddock et al., 1994; Zapert, 1994). 


\subsubsection{OMEGA (An Optimization Model for Economic and. Greenhouse Assessment)}

I propose to combine the economic component of DICE (equations 4.1-4.5) and the impact factor (equation 4.10) with the mathematical system formulation of IMAGE $1.0^{2}$. To be more precise, equations $4.6-4.9$ from the DICE model are discarded and replaced by the IMAGE 1.0 framework. This means that the emissions of fossil $\mathrm{CO}_{2}\left(\mathrm{u}_{13}\right)$ are equal to $\mathrm{y}_{4}(\mathrm{t})$. Considering the additional difference that I will now only consider fossil $\mathrm{CO}_{2}$ emissions for $\mathrm{y}_{4}(t)$ and, therefore, $I$ adopt a rescaled factor for $b_{1}$ based on Nordhaus (1991b). Non-fossil $\mathrm{CO}_{2}$ and CFCs emissions are included within the framework of IMAGE 1.0.

Furthermore, because $x_{141}(t)$ represents the temperature of the mixed upper ocean layer and thus not the surface temperature above land, an additional term $x_{156}(t)$ is included which has the same meaning as expression $y_{7}(t)$ in the DICE model:

$$
x_{I S 6}(t)=\frac{f=x_{157}(t)-k \cdot x_{141}(t)}{f \cdot \lambda+k}
$$

which represents the atmospheric temperature increase relative to $1900 . \mathrm{x}_{157}(\mathrm{t})$ is the total change in radiative forcing which can be derived after estimating the non- $\mathrm{CO}_{2}$ component, $x_{15 s}(t), k$ represents the heat transfer between land and oceans, $f$ is the fraction of the globe covered by land $(=0.3)$ and $\Delta \mathrm{T}_{2 \times \mathrm{CO} 2}$ the temperature increase for a doubled $\mathrm{CO}_{2}$ concentration $\left(=2.5^{\circ} \mathrm{C}\right)$.

$$
x_{15}(t)=\frac{\lambda \cdot \Delta T_{2 \times O_{2}}}{\ln (2)} \cdot \ln \left(\frac{\left.x_{13}(t)\right)}{x_{13}(1900)}\right)+x_{158}(t)
$$

2 Notice that MMAGE 1.0 as well as DICE do not contain scientific findings of the last years such as the role of CFC and sulphate aerosols. The results presented in this Chapter are an academic exercise showing the possibility of including more advanced descriptions of the climate system and its consequences on conclusions of economic cost estimates. In Chapter 6 optimization experiments are discussed using a more up to date global integrated assessment model TARGETS. 


$$
\begin{aligned}
& X_{I S S}(t)=a_{C F C H} \cdot x_{I U}(t)+a_{C F C I I} \cdot X_{I S}(t)+a_{C F C I I} \cdot X_{\text {IU }}(t) \\
& +a_{\text {croris }} \cdot x_{145}(t)+a_{\text {crocis }} \cdot x_{146}(t)+a_{H, O} \cdot\left(\sqrt{x_{150}(t)}-\sqrt{X_{153}(1900)}\right) \\
& +a_{C H} \cdot\left(\sqrt{X_{I S O}(t)}-\sqrt{x_{I S O}(1900)}\right) \\
& \left.-f\left(x_{I S O}(t), x_{I S 4}(1900)\right)+f\left(x_{i S S}(1900)\right)_{X_{I S 4}}(1900)\right) \\
& +a_{N, O} \cdot\left(\sqrt{x_{\text {ISA }}(t)}-\sqrt{x_{154}(1900)}\right) \\
& -f\left(x_{150}(1900), x_{154}(t)\right)+f\left(x_{150}(1900), x_{154}(1900)\right)
\end{aligned}
$$

where $f\left(x_{\text {ISI }}(t), x_{\text {ISA }}(t)\right)=$

$$
\begin{aligned}
& 0.47 \ln \left[1+a_{1} \cdot 10^{3} \cdot\left(x_{1.50}(t) \cdot x_{\text {ISU }}(t)\right)^{0.75}\right. \\
& \left.+a_{2} \cdot 10^{3} \cdot x_{1.54}(t) \cdot\left(x_{\text {ISS }}(t) \cdot x_{1.54}(t)\right)^{1.52}\right]
\end{aligned}
$$

where $\mathrm{a}_{i}$ are radiative forcing coefficients of the greenhouse gas $i$, and where the climate sensitivity parameter $\lambda$ is defined as a $4.3 \mathrm{~W} / \mathrm{m}^{2}$ increase of radiative forcing for a doubled $\mathrm{CO}_{2}$ concentration divided by the temperature increase for a doubled $\mathrm{CO}_{2}$ concentration is $4.3 / \Delta \mathrm{T}_{2 \times \mathrm{CO}}$.

The state space of the combined model can now be written as $\left(\mathrm{y}_{1}, \ldots, \mathrm{y}_{5}, \mathrm{x}_{\mathrm{r}},, \mathrm{x}_{158}, \mathrm{y}_{10}\right)$ and is controlled by $z_{1}$ and $z_{2}$ (Figure 4.1). OMEGA was developed in the modelling environment and simulation language M (Bruin et al., 1996), which is ultimately' converted into $C$. The system is solved using $2^{\text {nd }}$ order Runge-Kutta with a fixed time step of 0.05 years (Zapert, 1994). 


\subsection{Optimization Methodology}

\subsubsection{Problem Formulation}

The generic optimal control problem which can be associated with the coupled model has the following form:

$$
\begin{gathered}
\max _{z(t)} \int_{t_{0}}^{t} f(\mathrm{x}(t), \mathrm{y}(t), \mathrm{u}(t), \mathrm{z}(t)) d t \\
\text { s.t. } \\
\mathrm{z}(t) \in \mathcal{I}=\left\{\mathrm{z}(t) \in R^{2}: g(\mathrm{x}(t), \mathrm{y}(t), \mathrm{u}(t), \mathrm{z}(t))\right) \leq 0 \\
\left.\frac{d(\mathrm{y}(t), \mathrm{x}(t))}{d t}=F(\mathrm{y}(t), \mathrm{x}(t))+(\mathrm{u}(t), \mathrm{z}(t))\right\}
\end{gathered}
$$

where the objective is to maximize $\mathrm{f}(\mathrm{t})$, the discounted sum of consumption, in order to satisfy $\mathrm{g}($.). The input/control can be split into decision variables of the optimization problem $z_{1}(t)$ and $z_{2}(t)$ and the remaining input variables $u^{(5)}$ and $u^{(8)}$ which follow prescribed scenarios as given in Rotmans (1990).

The problem described in (4.17) is a continuous dynamic control problem. Due to the dimensionality of the problem it may be practically impossible to find an analytical solution. If the time steps are rendered discrete, the problem can be. transformed into an ordinary large scale non-linear problem, which can be solved using various numerical optimization techniques, within the limitation of these: methods. The general problem formulation is, therefore, stated as

$$
\begin{gathered}
\max f(z) \\
z \in R^{n}
\end{gathered}
$$

\section{subject to}

$$
g_{i}(\mathrm{z}) \leq 0 \quad i=1, \ldots, m
$$

For simplicity, the levels of the decision variables are assumed to change linearly over fixed time intervals during the period under consideration (1990-2100). The following time intervals are used: $\left[T_{0}, T_{1}\right], \ldots,\left[T_{10}, T_{11}\right], T_{0}=1990, T_{1}=2000, \ldots$, $T_{11}=2100$. The investments and emission control rates are assumed to change in a. 
linear fashion during $\left[\mathrm{T}_{k}, \mathrm{~T}_{\mathrm{k}+1}\right]$ where the investments and control rate levels in years $T_{k}$ and $T_{k+1}$ are denoted by, respectively, $\mu_{1, k}$ and $\mu_{2, k}$. The choice of $\mu_{1}=\left[\mu_{1, x}\right]_{k=1}{ }^{11}$ and $\mu_{2}=\left[\mu_{2, k}\right]_{k=1}$ " together with the known value of the initial values $u_{1,1990}$ and $u_{2,1990}$, determine $\mu_{1, k}$ and $\mu_{2, x}$. The $\mu_{1, k}$ 's and $\mu_{2, k}$ 's can be considered as the decision variables which determine $\mathbf{z}$.

In the next Section, I will introduce a new optimization approach, sequential reduced system programming, which aims at solving large scale optimization problems. I will compare its performance with several existing approaches which are briefly described in Section 4.3.3.

\subsubsection{Sequential Reduced-System Programming}

This method is specifically designed to enable the optimization of large-scale dynamical systems. Here the system is reduced to a smaller-scale representing only the core of the original system (Janssen and Vrieze, 1996). This technique is related to the two-step algorithm designed to solve the problem of combined identification and dynamic optimization as identified by Haimes and Wismer (1972), Ellis and Roberts (1982) and Ferreira and Borges (1994) in that sequentially the dynamical system and a dynamic optimization problem for a reduced version of the system is solved.

\section{Problem Formulation}

For practical reasons, a simplified problem formulation is used to discuss SRSP. Dynamical systems will be considered which can be written as the following set of differential equations:

$$
\frac{d \mathrm{x}(t)}{d t}=h(\mathrm{x}(\mathrm{z}), \mathrm{z})=\mathrm{A} \cdot \mathrm{x}(t)+\mathrm{N}(\mathrm{x}(t))+F(\mathrm{z}(t))
$$

where $x(t) \in R^{2}$ is the state vector of the system which describes all the characteristic quantities, $A$ is a square matrix and $F$ is a vector valued function of $z(t) \in R^{n}$, the input vector of the system. $N$ represents the non-linear terms. If $A, N, x_{0}$ and $z(t)$ for every $t$ are known then $\mathbf{x}(t)$ is assumed to be uniquely determined. Clearly $\mathbf{A}, \mathbf{N}$ and $\mathbf{x}_{0}$ are defined once and for all and $\mathbf{x}(\mathrm{t})$ is a function of $\mathbf{z}(\mathrm{t}): \mathbf{x}(\mathrm{z}(\mathrm{t}))$,

Suppose that the aim is to optimize the behavior of the system. Therefore, the elements of the input vector $z(t)$ have to be chosen so that an objective function $f: R^{s+n}$ $\rightarrow \mathrm{R}$ is maximized taking into account the $\mathrm{k}$ constraints represents by the vector valued function $g: R^{r+n} \rightarrow R^{k}$. Vector $z(t)$ can thus be considered as the decision vector. 
The optimal control problem can mathematically be stated as below

$$
\begin{gathered}
\max \int_{z(t)}^{t} f(\mathrm{x}(\mathrm{z}(\mathrm{t})), \mathrm{z}(t)) d t \\
\mathrm{z}(t) \in \mathrm{S}=\left\{\mathrm{z}(t) \in R^{n}: \mathrm{g}(\mathrm{x}(\mathrm{z}(\mathrm{t})), \mathrm{z}(\mathrm{t})) \leq 0\right. \\
\left.\frac{d \mathrm{x}(t)}{d t}=\mathrm{A} \cdot \mathrm{x}(\mathrm{t})+\mathrm{N}(\mathrm{x}(\mathrm{t}))+F(\mathrm{z}(t))\right\}
\end{gathered}
$$

where $\Im \in R^{n}$ is the decision space. All functions are supposed to be continuously differentiable.

\section{Algorithm}

The algorithm which can solve (4.20) is based on the idea to reduce the system (4.19) into a smaller system, fixing certain variables, and which allows the application of optimization methods in order to derive an optimal solution for this smaller system. Next, this solution is used as input for the next iteration of the original system, etc.

I will now split up the state space into $x^{\prime}(t) \in R^{m}$ and $x^{2}(t) \in R^{s m}$, with $m<s$. The algorithm will treat the variables $x^{\prime}(t)$ as normal endogenous and $x^{2}(t)$ as given exogenous values which are updated every iteration. Observe that, for the small system, $x^{1}(t)$ is steered by the input variable $z(t)$, and the fixed input $x^{2}(t)$. The other part of the system, $x^{2}(t)$, is only influenced indirectly by changes in the input variable $z(t)$ through $x^{\prime}(t)$

The resulting algorithm is therefore:

Choose some start vector $\mathrm{z}_{0}(\mathrm{t})$. Set $\mathrm{k}=1$.

Step 1 (Simulation) Using $z_{k-1}(t)$ solve

$$
\frac{d \mathrm{x}(t)}{d t}=\mathrm{A} \cdot \mathrm{x}(t)+\mathrm{N}(\mathrm{x}(t))+F\left(\mathrm{z}_{\mathrm{k}-l}(t)\right)
$$

to derive $x_{k}(t)=\left\{x_{k}^{1}(t), x_{k}^{2}(t)\right\}$. 
Step 2 (Optimization) Fixing the estimates of $x_{k}^{2}(t)$ from step 1, obtain $z_{k}(t)$ by solving

$$
\begin{aligned}
& \max _{z(t)} \int_{t_{\theta}}^{t} f\left(\mathrm{x}^{1}(\mathrm{z}(\mathrm{t})), \mathrm{z}(t)\right) d t \\
& \mathrm{z}(t) \in \mathfrak{I}_{k}=\left\{\begin{array}{l}
z(t) \in R^{n}: g\left(x^{\prime}(z)(t), z(t)\right) \leq 0 \\
\frac{d x^{\prime}(t)}{d t}=A_{1} \cdot\left(\begin{array}{l}
x^{1}(t) \\
x_{k}^{2}(t)
\end{array}\right)+N_{1}\left(x^{1}(t), x_{k}^{2}(t)\right)+F_{1}(z(t))
\end{array}\right\}
\end{aligned}
$$

Set $\mathrm{k}=\mathrm{k}+1$ and go to Step 1 .

In step 2 of the algorithm $A_{1}$ is the appropriate submatrix of $A$, while $N_{1}$ and $F_{1}$ refer to the $\mathrm{x}^{\prime}$-components of $\mathrm{N}$ and $\mathrm{F}$ respectively. The steps of simulation and optimization are performed sequentially until no further improvement can be observed, or until the convergence criterion is met.

\section{Convergence of the Algorithm}

The algorithm generates a sequence $x_{k}^{2}(t)$ and $z_{k}(t), k=0,1,2, \ldots$. Some conditions have to be fulfilled in order to guarantee the convergence of this sequence. Since $x_{k}(t)$ is dependent on $z_{k-1}(t)$ and $z_{k}(t)$ on $x_{k}(t)$, the solution $x_{k}^{2}(t)$ of the system (4.21) can be substituted into the problem formulation (4.22). Therefore, a map $M: R^{n} \rightarrow R^{n}$ can be defined which represents the relation between $z_{k}$ and $z_{k-1}$, or $z_{k}=M\left(z_{k-1}\right)$ where $M$ denotes the optimal solution of problem (4.22) using the solution $x_{k}^{2}(t)$ of system (4.21). Obviously, any convergence point of algorithm coincides with a fixed point of $\mathrm{M}$. On the other hand, it can easily be seen that any optimal solution of the system (4.20) also coincides with a fixed point of M. Let $z \cdot(t)$ be a fixed point of M. I assume that if $\mathbf{z}_{0}(\mathrm{t})$ is started in the attraction space $\mathfrak{J}^{*}$ of $\mathbf{z} \cdot(\mathrm{t})$, that $\mathrm{M}$ is a continuous map.

The map $M$ is a contraction map on a compact subspace $S \subset R^{n}$, whenever $M(z) \in$ $S$ for any $z \in S$ and ||$M\left(z_{1}\right)-M\left(z_{2}\right)|| \leq \lambda|| z_{1}-z_{2}||$ for any $z_{1}, z_{2} \in S$ and $\lambda \in(0,1)$ in some well defined norm || .| $\mid$. In such a case, $M$ has a unique fixed point on $S$. A sufficient condition for the existence of a fixed point is the following:

$$
\left|\frac{\left.\partial m_{i}(z)\right)}{\partial z}\right| \leq \frac{K}{n} \quad \text { whenever } z(t) \in \mathfrak{S}^{*}
$$

where $0<\mathrm{K}<1$ and for each $\mathrm{j}=1,2, \ldots \mathrm{n}$ and each component function $\mathrm{m}_{i}$ of $\mathrm{M}$. Then the sequence $\left\{z_{k}(t)\right\}$, defined by a starting vector $z_{0}(t)$ in the attraction set $S^{*}$ of $z \cdot(t)$ and generated by $z_{k}(t)=M\left(z_{k-1}(t)\right)$ converges to the unique solution $z_{r} \cdot(t)$. 
Condition (4.23) can be rewritten as

$$
\left|\sum_{m=i}^{m} \frac{\partial m_{i}\left(z^{\prime}(t)\right)}{\partial x^{2}(w, t)} \cdot \frac{\partial x^{2}(w, t)}{\partial z_{i}^{t}(t)}\right| \leq \frac{K}{n},
$$

where $x^{2}(w, t)$ denotes the $w$-th component of the vector $x^{2}(t)$. Inequality (4.24) says that if $x^{2}(t)$ is not "too sensitive" to changes in the decision variable $z(t)$ and if an optimal $\mathrm{z}$ of the problem (4.22) for a new iteration is not "too sensitive" to changes in $x^{2}(t)$, then the algorithm will converge. Translated back to the separation of the state vector $\mathrm{x}$ into $\mathrm{x}^{1}$ and $\mathrm{x}^{2}$ this means that $\mathrm{x}^{1}$ should be chosen so that it represents the core dynamics of the model.

Although the above conditions can hardly be checked for large-scale problems, it points to the fact that if the solution of an iteration does not largely change in comparison to the solution of the preceding iteration, the algorithm converges to $\mathrm{z} \cdot(\mathrm{t})$. However, if $\mathbf{z}_{k}(t)$ converges, the solution will not necessarily be a global or even a local optimum of (4.20). Conditions for a local optimum will be formulated in the next paragraph.

\section{Convergence of the Optimal Solution}

If $\mathbf{z} *$ is a fixed point of the algorithm $M$ which generates the system state $\mathbf{x}$, what are the conditions for $z$ to be a (local) optimum of (4.20)? The following equation of the Hamiltonian of problem (4.20) can be formulated.

Define

$$
H(\mathrm{x}, \mathrm{z}, \lambda)=f(\mathrm{x}(\mathrm{z}), \mathrm{z})+\sum_{i=1}^{s} \lambda_{i} h_{i}(\mathrm{x}(\mathrm{z}), \mathrm{z})+\sum_{i=1}^{k} \mu_{i} g_{i}(\mathrm{x}(\mathrm{z}), \mathrm{z})
$$

where $\lambda(t)=\left(\lambda_{1}(t), . ., \lambda_{1}(t)\right)$ and $\mu(t)=\left(\mu_{1}(t), \ldots, \mu_{k}(t)\right)$ are assumed to be continuously differentiable functions and where $h_{i}$ is the $i^{\text {th }}$ equation of the dynamical system.

If the vector control function $z^{*}$ and the corresponding state vector $x^{*}$ soive problem (4.20), then $z^{*}, x^{*}, \lambda, \mu$ together simultaneously should satisfy

$$
\begin{aligned}
& \frac{d \mathrm{x}(t)}{d t}=\frac{d H}{d \lambda}=h(\mathrm{x}(\mathrm{z}), \mathrm{z}) \\
& g(\mathrm{x}(\mathrm{z}), \mathrm{z}) \leq 0
\end{aligned}
$$




$$
\begin{aligned}
& 0=\frac{\partial H}{\partial z_{j}}=\frac{\partial f}{\partial z_{j}}+\sum_{i=1}^{j} \lambda_{i} \frac{\partial h_{i}}{\partial z_{j}}+\sum_{i=1}^{k} \mu_{i} \frac{\partial g_{i}}{\partial z_{j}}, \quad j=1, \ldots, n \\
& \frac{d \lambda_{j}}{d t}=-\frac{\partial H}{\partial x_{j}}=-\left(\frac{\partial f}{\partial x_{j}}+\sum_{i=1}^{j} \lambda_{i} \frac{\partial h_{i}}{\partial x_{j}}+\sum_{i=1}^{k} \mu_{i} \frac{\partial g_{i}}{\partial x_{j}}\right), j=1, \ldots, s \\
& \mu \geq 0, \quad \mu g=0
\end{aligned}
$$

It is clear that if $\mathrm{m}=\mathrm{s}$, in case that the system is not reduced, the solution found by the algorithm is equal to a local optimum satisfying the necessary conditions as above. It might occur that, in case $\mathrm{m}<\mathrm{s}$, a convergence point of the algorithm does not coincide with a local optimum. I will trace a set of sufficient conditions of the reduced system for which a solution within an area of tolerance around a local optimum is found by SRSP.

In a fixed point $z^{*}$ of $M$ a local optimum is found for problem (4.22), so conditions (4.26), (4.27) and (4.30) are satisfied.

If $\mathrm{g}$ and $\mathrm{f}$ do not directly depend on $\mathrm{z}$ and if $\mathrm{F}(\mathrm{z})=\mathrm{B} \mathbf{z}$, where $\mathrm{B}$ is a (sxn)-matrix of coefficients, I can derive

$$
\begin{aligned}
& \frac{\partial f}{\partial z_{i}}=0, \quad \text { for } j=1, \ldots, n \\
& \frac{\partial g_{i}}{\partial z_{j}^{*}}=0, \quad \text { for } j=1, \ldots, n \text { and } \text { for } i=1, \ldots, k \\
& \frac{\partial h_{i}}{\partial z_{j}^{*}}=b_{i} \quad \text { for } i=1, \ldots s \text { and } j=1, \ldots n
\end{aligned}
$$


While in the local optimum of (4.22) there are $\lambda_{i}{ }^{\circ}$ and $\mu_{i}{ }_{i}, i=1, \ldots, m$, such that

$$
\frac{\partial f}{\partial z_{j}^{*}}+\sum_{i=1}^{m} \lambda_{i}^{*} \frac{\partial h_{i}}{\partial z_{j}^{*}}+\sum_{i=1}^{k} \mu_{i}^{\cdot} \frac{\partial g_{i}}{\partial z_{j}^{*}}=0 \text { for } j=1, \ldots, n
$$

It follows that

$$
0=\sum_{i=1}^{m} \lambda_{i}^{*} \cdot b_{i j}, \quad j=1, \ldots, n
$$

An obvious solution to (4.35) is the time independent choice $\lambda_{i}{ }^{*}=0, i=1, \ldots, m$. Observing the fact that $\mathrm{d} \lambda_{\mathrm{i}} / \mathrm{dt}=0$ for $\mathrm{i}=1, \ldots, \mathrm{m}$ it follows that from the optimality conditions of problem (4.20), I obtain

$$
0=-\left(\frac{\partial f}{\partial x_{j}^{*}}+\sum_{i=1}^{m} \lambda_{i} \frac{\partial h_{i}}{\partial x_{j}^{*}}+\sum_{i=1}^{k} \mu_{i}^{*} \frac{\partial g_{i}}{\partial x_{j}^{*}}\right), j=1, \ldots, n
$$

in the fixed point. Consequently equation (4.29) can now be reduced to

$$
0=\sum_{i=m+l}^{s} \lambda_{i} \frac{\partial h_{i}}{\partial x_{i}^{*}}, \quad j=1, \ldots, n
$$

Finally

$$
\frac{d \lambda_{j}}{d t}=-\left(\frac{\partial f}{\partial x_{j}^{*}}+\sum_{i=m+l}^{t} \lambda_{i} \cdot \frac{\partial h_{i}}{\partial x_{j}^{*}}+\sum_{i=1}^{k} \mu_{i} \frac{\partial g_{i}}{\partial x_{j}^{*}}\right) j=n+1, \ldots, s
$$


which can be written as

$$
\begin{gathered}
\frac{d \lambda_{i}}{d t}=\sum_{i=m+1}^{t} a_{i j}(t) \cdot \lambda_{j}+b_{j}(t), j=n+1, \ldots, s \\
\text { with } \\
a_{i j}(t)=-\frac{\partial h_{i}}{\partial x_{i}} \\
b_{j}(t)=-\frac{\partial f}{\partial x_{j}}-\sum_{i=1}^{k} \mu_{i} \frac{\partial g_{i}}{\partial x_{j}}
\end{gathered}
$$

According to Kamien and Schwartz (1981, page 313), there is a unique solution for the (s-n) differential equations if the derivatives and $\mu_{1}^{\prime} s$ are continuous on the considered time horizon. Therefore, I may conclude that if $\mathrm{g}$ and $\mathrm{f}$ do not directly depend on $\mathbf{z}$, if the control is linear $\mathrm{F}(\mathrm{z})=\mathbf{B} \mathbf{z}$, and if conditions (4.37) and (4.38) are fulfilled, then a solution can be found which satisfies (4.26)-(4.30) for this class of problems using SRSP. Notwithstanding, it can be proved that SRSP can successfully be applied to a large class of problems, it might occur that in practice problems do not satisfy the conditions in order to be certain of nice convergence properties. However, in some cases, SRSP still appears to be a suitable algorithm, as will be shown in the next case study for OMEGA.

\section{Case Srudy}

The optimization problem is somewhat simplified to show the performance of SRSP compared with a traditional approach, a quasi-Newton approach. The emissions of fossil fuels are defined as a function of economic output $\mathrm{y}_{1}(\mathrm{t})$ times a time dependent emission quotient per unit of output, $\sigma(t)$, and a forcing term to start emission reduction policy.

$$
y_{5}(t)=\frac{\alpha+1}{\alpha+e^{t(t \cdot 1090)}} \cdot \sigma(t) \cdot y_{l}(t)
$$

I will discuss to test problems: an unconstrained cost-benefit analysis (test case 1), and an analysis where a maximum temperature target of $2^{\circ} \mathrm{C}$ is used (test case 2) (see also Section 4.4). 


\section{Results}

The original model consists of 163 equations, but has been reduced to a system of 9 essential equations (see Appendix 4.1). Because the optimization problem only consists of one decision variable, the exact solutions can be traced. In Figure 4.2 and 4.3, the relations are plotted between the decision variable and the value of the objective function and the maximum temperature increase. The optimal solutions for the test cases are $z=0.00950$ (case 1 ) and $z=0.02955$ (case 2).

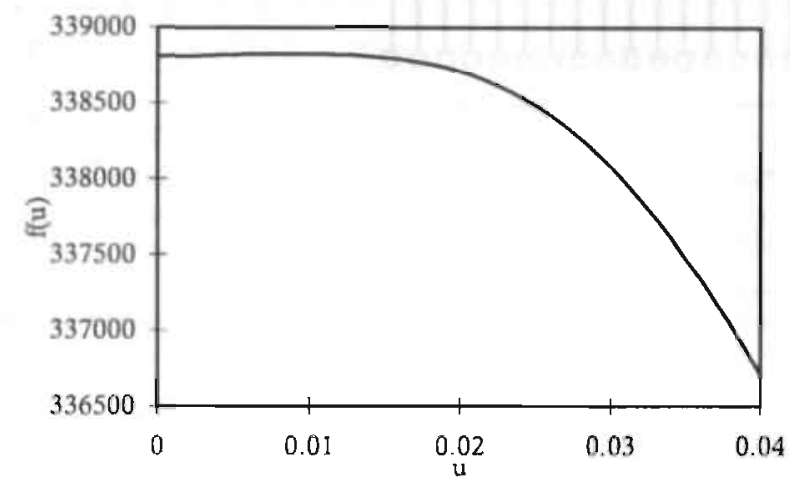

Figure 4.2: Value of the objective function $\mathrm{flu}$

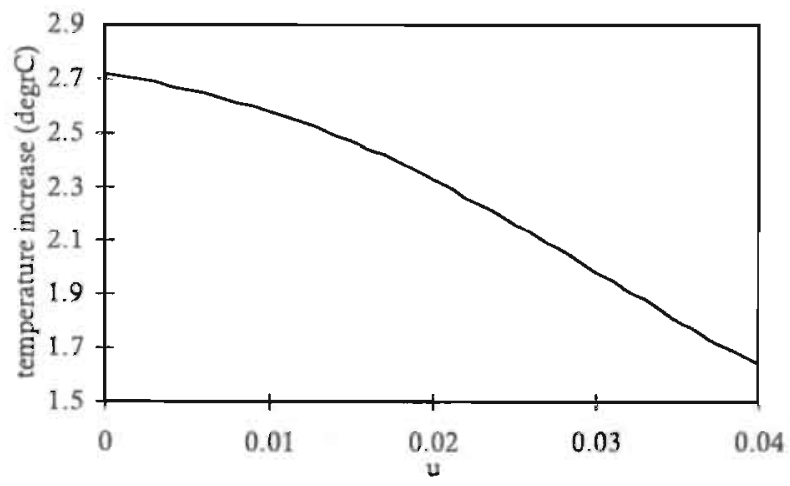

Figure 4.3: Maximum temperature increase for the next century as a function of $u$. 


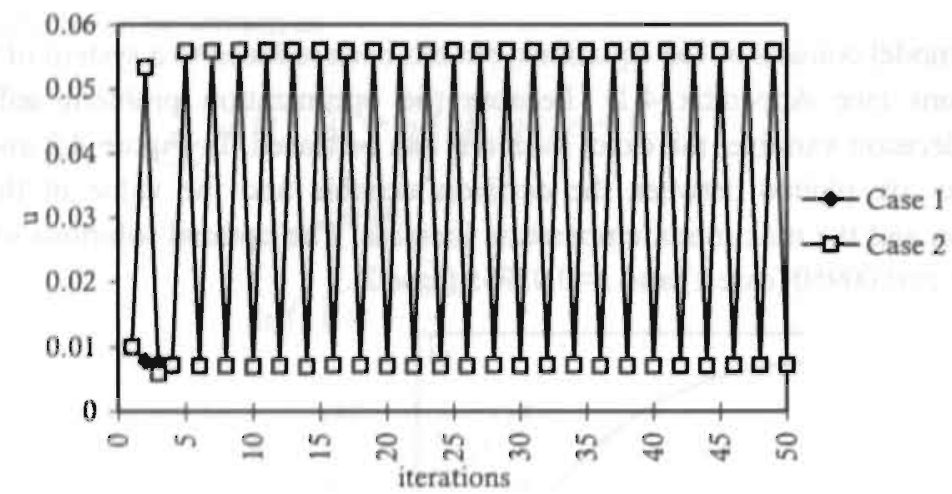

Figure 4.4: Objective value during running the algorithm.

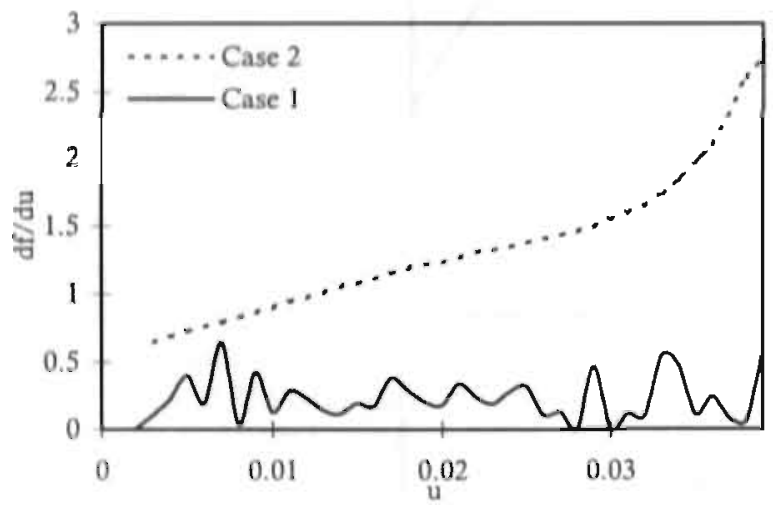

Figure 4.5: Condition (4.23).

If the original SRSP code is used to solve two test problems, the algorithm converges only for test case 1 (see Figure 4.4). Checking condition (4.23) of the algorithm leads to the conclusion that SRSP applied on test case 2 do not result in a value of condition (4.23) below one instead of SRSP applied on test case 1 (Figure 4.5) where the derivative is smaller than one for the whole range of $z$. However, in order to find a fixed point of the algorithm, I introduce a heuristic solution to avoid divergence from the fixed point:

Known is that for every $\mathrm{k}$

$z_{k-1}=\alpha z_{k-2}+B$

$z_{k}=\alpha z_{k-1}+B$

While in the fixed point $z^{*}=\alpha z^{*}+B$ the following equation can be derived 
$\alpha=\left(z_{k}-z_{k-1}\right) /\left(z_{k-1}-z_{k-2}\right)$

$B=z_{k-1}-\alpha z_{k: 2}$

Expected value of $z^{*}=B /(1-\alpha)$

So, the heuristic adaptation of the algorithm will be the following check after optimizing problem (4.22):

if $\mathrm{k}=1 \mathrm{z}=\mathrm{z}$;

if $\mathrm{k}>=2 \mathrm{z}_{2}=\mathrm{z}_{1}, \mathrm{z}_{1}=\mathrm{z}$;

if $\mathrm{k}>=3\left\{\mathrm{z}_{3}=\mathrm{z}_{2} ; \mathrm{z}_{2}=\mathrm{z}_{1}, \mathrm{z}_{1}=\mathrm{z}\right\}$

if ||$z_{2}-z_{1}||<|| z_{3}-z_{2}||$ then $\left\{\right.$ set $z_{3}$ to the expected value of $z^{*}$ and $\left.k=1\right\}$

This adaptation forces the algorithm to a fixed point. Figure 4.6 shows the results of SRSP improved when the solution is forced to a fixed point. Table 4.1 shows the results of the algorithm of the two cases compared to the optimal solution and to a usual quasi-Newton algorithm for solving problem (4.22):

\begin{tabular}{||c|l|l|l|l|l|l|}
\hline & \multicolumn{3}{|c|}{ Test case 1 } & \multicolumn{3}{c|}{ Test case 2 } \\
\hline & \multicolumn{1}{|c|}{ SRSP } & \multicolumn{1}{|c|}{ Optimal } & q-Newton & \multicolumn{1}{|c|}{ SRSP } & \multicolumn{1}{|c|}{ Optimal } & q-Newton \\
\hline$z^{*}$ & 0.007644 & 0.0093 & 0.009189 & 0.029545 & 0.02955 & 0.029549 \\
$\left.\mathrm{f}^{*} \mathrm{z}^{*}\right)$ & 338824.19 & 338826.03 & 338826.03 & 338113.51 & 338118.97 & 338118.2 \\
time & 15.5 & & 1273.7 & 183.9 & & 3953.4 \\
$\# \mathrm{fe}$ & $3(79)$ & & 401 & $13(1608)$ & & 1228 \\
$\Sigma \mathrm{g}\left(\mathrm{z}^{*}\right)$ & & & & 0.000573 & 0.0 & 0.0 \\
$\mathrm{df}\left(\mathrm{z}^{*}\right) / \mathrm{dt}$ & 807.3 & 0.0 & 78.1 & & & \\
\hline
\end{tabular}

Table 4.1: Comparison of the optimal solutions of SRSP, quasi-Newton and the real solution of the two test cases. The run time is in seconds on a Silicon Graphics (Indy) Workstation. The number of function evaluations ( $\# \mathrm{fe}$ ) of the original systems are given next to the number of evaluating the reduced system which is given between brackets.

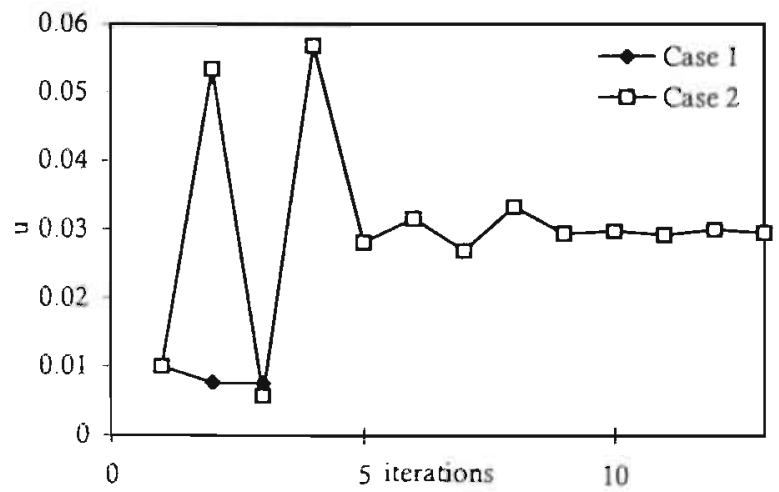

Figure 4.6: Objective function value by forced convergence. 


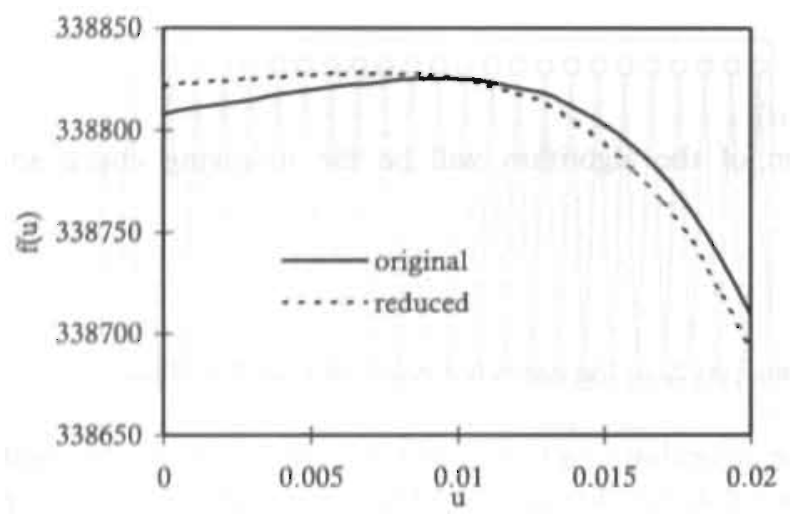

Figure 4.7: Original versus reduced model results .

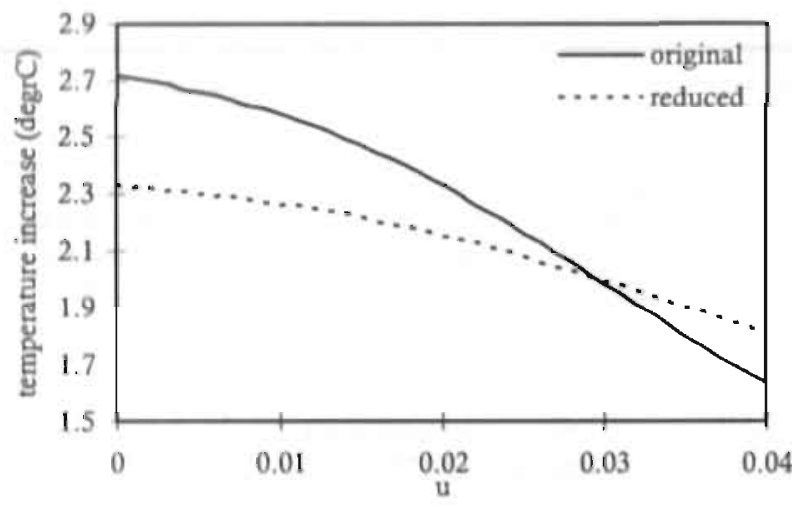

Figure 4.8: Original versus reduced model temperature change in 2100.

Because $\mathrm{F}(z)$ is a nonlinear function of $z$, convergence to a unique solution can not be proved. However, the algorithm finds a good, but not necessary, optimal solution of the original system. Figure 4.7 shows the relation between the objective value and the decision variable $z$ of the original problem and the reduced problem in the fixed point. The reduced problem is a little perturbed and reaches a maximum in a somewhat smaller value $(0.007644)$ than the original problem $(0.00950)$.

The approximation of the temperature increase (in 2100) in the fixed point of test case 2 is plotted in Figure 4.8 and is related to the real values of the original system. The approximation in the fixed point is perfect, but a perturbation of $z$ leads already to a significant difference with the actual values which may be the cause of divergence of the original version of SRSP. 


\subsubsection{Some other Optimization Approaches}

\section{The Penalty Method (PM)}

The penalty method (PM) transforms the constrained problem into an unconstrained problem by substituting a penalty function for the constraints. The idea is to penalize constraint violation by adding a sequence of penalty functions to the objective function in such a manner that the solutions to the resulting sequence of unconstrained problems tend to a constrained minimum (Luenberger, 1984).

This straightforward method can be formulated as an unconstrained problem of the form

$$
\min f(\mathrm{z})+c P(\mathrm{z})
$$

where $c$ is a positive constant and $\mathrm{P}$ is a continuous function on $\mathrm{R}^{\mathrm{n}}$ satisfying $\mathrm{P}(\mathrm{z}) \geq 0$ $\forall \mathrm{z} \in \mathrm{R}^{\mathrm{n}}$ and where $\mathrm{P}(\mathrm{z})=0$ if and only if $\mathrm{z}$ is a feasible solution.

To solve problem (4.41) by the penalty function method is to use a sequence $\left\{c_{k}\right\}$, $k=1,2, \ldots$, tending to infinity such that for each $k, c_{k} \geq 0, c_{k+1}>c_{k}$. Define the function $q(c, z)=f(z)+c P(z)$ and solve for each $k$ the following problem

$$
\min q\left(c_{k}, z\right)
$$

Z

to obtain a solution point $\mathrm{z}_{\mathrm{k}}$ assuming that, for each $\mathrm{k}$ problem (4.42) has a solution.

In this Chapter, the most widely used exact penalty function, the absolute-value penalty function, is applied. The absolute value penalty function may be written as

$$
q(\rho, \mathrm{z})=f(\mathrm{z})+\rho\left\|g(\mathrm{z})^{*}\right\|_{\mathrm{i}}
$$

This non-differential penalty function has the property that there is a finite threshold value $\rho^{*}$ such that $z^{*}$ is an unconstrained minimizer of $P_{1}$ for any $\rho>p^{+}$.

The direct set algorithm (Press et al., 1988) is used for the experiments to eliminate the need to calculate the derivative of the mathematical system in finding a local optimum. 


\section{The Sequential Quadratic Programming Method (SQP)}

The SQP method solves a sequence of quadratic sub-problems derived using the first and second order terms of the Taylor expansion of $\mathrm{f}(\mathrm{z})$ and linear approximations of the constraints $\mathrm{g}(\mathrm{z})$ (Nemhauser et al., 1989). SQP is based directly on the optimality conditions of nonlinear programming. Necessary conditions for $z^{*}$ to be a local minimum of (4.18) are the well-known Karush-Kuhn-Tucker (KKT) equations:

$$
\begin{array}{lr}
\nabla f\left(z^{*}\right)+\sum_{i=1}^{m} \lambda_{i}^{*} \cdot \nabla g_{i}\left(z^{*}\right)=0 \\
\lambda_{i}^{*} \cdot g_{i}\left(z^{*}\right)=0 & i=1, \ldots, m \\
\lambda_{i}^{*} \geq 0 & i=1, \ldots, m
\end{array}
$$

where $\lambda_{1}{ }^{*}$ are the Lagrangian multipliers.

The solution of the KKT equations forms the basis for the SQP. At each major iteration an approximation is made of the Hessian of the Lagrangian function using a quasi-Newton updating method, regarding information from the KKT equations. This approximation is then used to form a search direction for a line search procedure.

At each iteration of the method, a Quadratic Programming (QP) problem is solved using the Wolfe algorithm (Wolfe, 1959). The QP sub-problem is a quadratic approximation of the Lagrangian function,

$$
L(z, \lambda)=f(z)+\sum_{i=1}^{m} \lambda_{i} \cdot g_{i}(z)
$$

The QP sub-problem can then be obtained by linearizing the nonlinear constraints,

$$
\begin{aligned}
& \underset{\text { minimize }}{ } \frac{1}{2} \mathrm{~d}^{T} \mathrm{H}_{k} \mathrm{~d}+\nabla \mathrm{f}\left(\mathrm{z}_{k}\right)^{T} \mathrm{~d} \\
& \mathrm{~d} \in R^{n} \\
& \text { subject to: } \\
& \nabla g_{i}\left(\mathrm{z}_{k}\right)^{T} \cdot \mathrm{d}+g_{i}\left(\mathrm{z}_{k}\right) \leq 0 \quad i=1, \ldots, m
\end{aligned}
$$

where $d$ is the search direction. The matrix $H_{k}$ is a positive definite approximation of the Hessian matrix of the Lagrangian function and is updated by a positive-definite quasi-Newton approximation using the BFGS method (MATLAB, 1992). The solution procedure initially involves the calculation of a feasible point (if one exists), and then the generation of an iterative sequence of feasible points which achieve convergence to the solution. 


$$
\mathrm{z}_{k+1}=\mathrm{z}_{k}+\alpha_{k} \mathrm{~d}_{k}
$$

The nonnegative steplength parameter $\alpha_{k}$ is determined by an approximate line search procedure so that a sufficient decrease in a merit function is obtained.

Genetic Algorithm (GA)

The genetic algorithm is not designed explicitly as an optimization algorithm, as will be seen in Chapter 8 and 9, but has been developed by Holland (1975) by means of trying to abstract and explain the adaptive processes of natural systems. The basic construction is to consider a population of individuals that each represent a potential solution to a problem. The relative success of each individual on this problem is considered as its fitness, and is used to selectively reproduce the most fit individuals to produce similar, but not identical, offspring for the next generation (Figure 4.9). Genetic algorithms are mainly applied in optimization studied because they surpass their more traditional cousins in the quest for robustness (Goldberg, 1989).

Genetic algorithms are different from more normal optimization and search procedures in the following four ways (Goldberg, 1989):

1. GAs work with a coding of the parameter set, not the parameters themselves.

2. GAs search from a population of points, not a single point.

3. GAs use payoff (objective function) information, not derivatives or other auxiliary knowledge.

4. GAs use probabilistic transition rules, not deterministic rules. 


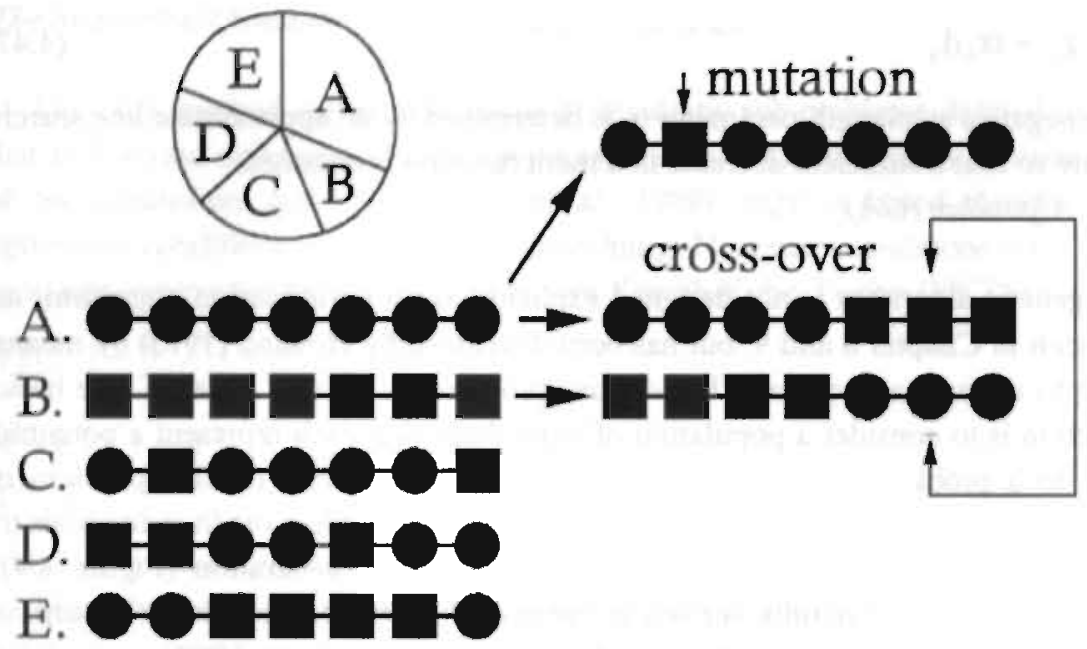

Figure 4.9: Schematic diagram of a genetic algorithm.

A formal description of the genetic algorithm is the following:

(1) An individual can be characterized by a binary bit string of fixed length $\mathrm{L}$, which is denoted as $a$, and $a \in B^{L}$ where $B=\{0,1\}$. The bit string can be separated into $n$ segments of equal length $\mathrm{l}_{z}$, thus implying $L=\mathbf{n} * 1_{z}$. Each segment is interpreted as the binary code of the object variable $\mathrm{z}_{\mathrm{i}} \in\left[\mathrm{z}_{\mathrm{i}}^{1}, \mathrm{z}_{\mathrm{u}}^{\mathrm{u}}\right]$ which can be redecoded by applying:

$$
\Gamma\left(a_{i l} \ldots a_{i l}\right)=z_{i}^{i}+\frac{z_{i}^{u}-z_{i}^{l}}{2^{t}-1} \cdot\left(\sum_{j=0}^{l} a_{i\left(t_{t}-j\right)} \cdot 2^{l}\right)
$$

where $\left(a_{i 1} \ldots a_{i 2}\right)$ denotes the $i$-th segment of an individual $a \in B^{L}$. Then $\Gamma=\Gamma_{1} x \ldots x \Gamma_{n}$ yields a vector of real values on the desired range $\left[z_{i}^{i}, z_{i}^{\prime \prime}\right]$.

Example: $\quad a=10011, z^{1}=0, z^{\mathrm{u}}=1$

$$
\Gamma=\left(1 * 2^{4}+0^{*} 2^{3}+0^{*} 2^{2}+1 * 2^{1}+1^{*} 2^{9}\right) / 31=0.613
$$

(2) Mutation is a bit reversal event that occurs with small probability $p_{m}$ per bit. This mutation can explore new genetic information and is a powerful operator in finding ways to adapt to a changing environment.

Example: Suppose I have the following bit string:

At random, roughly one in every 1000 symbols flips from 0 to 1 or vice versa; in the example from 1 to 0 :

11011 
(3) The algorithm uses a crossover operator that exchanges substrings arbitrarily between two individuals with probability $\mathrm{p}_{\mathrm{c}}$. Length and position of these substrings are chosen at random, but are identical for both individuals.

Example: Suppose I have the following bit strings: 11111 and 00000

A point along the strings is selected at random and the offspring

contain a mixture of the parents: $\quad 11000$ and 00111

(4) The probabilistic selection operator forms the next generation by copying individuals on the basis of fitness-proportionate probabilities

$$
p_{i}=\frac{F\left(a_{i}\right)}{\sum_{i=1}^{N} F\left(a_{j}\right)}
$$

where $F: B L R$ is the fitness function. The less fit individuals are therefore less likely to reproduce their genetic information.

According to Goldberg (1989) genetic algorithms are successful robust algorithms in optimization because they are able to select strings with useful blocks of information, and concentrate their search (selection) on variations which include those blocks. In complex problems, finding a good solution often depends upon putting together several of such useful blocks in the right combination. Genetic algorithms can be a powerful and relatively robust algorithm for searching in mathematical spaces defined by (nonlinear and nondifferential) functions of muitiple variables. Although, there is no convergence criterion and we don't know if the algorithm has found a global optimum, in many practical applications, it is often satisfactory to find a good solution, better than the solution we started.

I may start with a set of initial solutions lying in a predetermined range $\left[z_{i l}{ }^{1}, d_{i l}{ }^{u}\right]$. This approach may be used to incorporated a priori knowledge. The consequence of this approach is that independent of the degree of optimality, solutions found are improvements on present or proposed scenarios.

In the following Sections, I will discuss some experinnents performed with OMEGA. First I will discuss the problem formulation, thereafter the results and the performance of the various optimization algorithms. Finally, I will discuss differences found between OMEGA and DICE experiments. 


\subsection{Analysis}

Since climate change policy has numerous objectives, any formulation of an optimization problem will necessarily neglect some specific aspects, and the problems which are examined in this study can, therefore, only be seen as possible illustrative examples in climate policy. The analysis concerns the investigation of three problems with two starting points. Investments are assumed to be at an equal level at each starting point, all of which are based on optimal strategies in the absence of environmental policy. I propose to cover the range of possible policy options based on IPCC scenarios (IPCC, 1991), by referring to two levels of emission reductions:

- Business-as-Usual (BaU). a continuation of current trends.

- Accelerated Policies (AP) a shift towards non-fossil fuels is adopted.

Furthermore, I distinguish two scenarios for $\mathbf{u}^{(\delta)}$ (land use change) and $\mathbf{u}^{(\infty)}$ (other than fossil $\mathrm{CO}_{2}$ emissions): $\mathrm{BaU}$ and AP. This leads to four start scenarios, whereby the resulting temperature increase in 2100 compared to 1900 is given in Table 4.2. If the Business-as-Usual policy is followed for all sources, it is expected that temperature will increase by some 4 degrees Celsius. However, if fossil $\mathrm{CO}_{2}$ as well as other sources, are controlled according to the Accelerated Policies scenario the temperature increase will be limited to 1.3 degrees Celsius. Other starting points lead to the following global-mean temperature increases in 2100 compared to 1900 for the various cases:

Table 4.2: Global-mean temperature increase $x_{156}$ in 2100 as projected by OMEGA (in ${ }^{\circ} \mathrm{C}$ ).

\begin{tabular}{|l|c|c|}
\hline$z \backslash u^{(9)}, u^{(8)}$ & $\mathrm{BaU}$ & $\mathrm{AP}$ \\
\hline $\mathrm{BaU}$ & 3.7 & 2.7 \\
\hline $\mathrm{AP}$ & 2.3 & 1.3 \\
\hline
\end{tabular}

The purpose of the considered optimization problems is to control $z$, given $u$, to maximize the discounted sum of utility of future consumption given constraints on environmental impacts. Based on Nordhaus $(1992 ; 1993 ; 1994)$, I propose to use the following problem formulations:

\section{1) Cost-benefit}

This case is a straightforward cost-benefit optimization problem. The impacts of emission reduction cost and damage cost of a temperature increase are included in the objective function. The solution to this problem is an economically-efficient policy designed to slow climate change. 


\section{2) Maximum concentration of $\mathrm{CO}_{2}$.}

In order to appraise the effects of anthropogenic emissions, the increase concentration of greenhouse gases is limited. In several studies, such targets have been used in developing response scenarios (e.g. Krause et al., 1989; IPCC, 1991). Translated to $\mathrm{CO}_{2}$ concentration an upper limit, $c_{\max }$ of $400 \mathrm{ppmv}$ (Krause et al, 1989) will be used.

$$
x_{33}(t) \leq c_{\max }
$$

\section{3) Maximum temperature increase.}

From an environmental perspective, UNEP's Advisory Group on Greenhouse Gases (AGGG, 1990) has identified several climate targets in order to protect the structure and functions of vulnerable ecosystems and to limit risks for society. Such a target might be an absolute temperature limit of $2^{\circ} \mathrm{C}$ above pre-industrial level. This temperature limit can be viewed as an upper limit, beyond which risks of considerable damage are expected to increase rapidly.

$$
x_{158}(t) \leq \Delta T_{\max }
$$

\subsection{Results}

\subsubsection{Optimal Responses}

\section{Emissions}

In the case of the optimal cost-benefit formulation, the emissions of fossil $\mathrm{CO}_{2}$ rise to a level of $16 \mathrm{GtC}$ in 2100 which is about $10 \mathrm{GtC}$ above the present level (Figure 4.10). To meet the $\mathrm{CO}_{2}$ concentration target, the emissions stabilize at a level of 5 $\mathrm{GtC}$. The control of non- $\mathrm{CO}_{2}$ gases does not influence the optimal policy of the first two problem formulations. However, including a temperature target in the search for suitable future greenhouse policy ieads to large differences in emission reductions, whether emissions of other gases are reduced or not.

In the event that emissions of greenhouse gases other than fossil $\mathrm{CO}_{2}$ are not reduced, fossil $\mathrm{CO}_{2}$ has to be phased out by the end of the next century in order to meet the $2{ }^{\circ} \mathrm{C}$ temperature increase target. An additional reduction in emissions of other greenhouse gases results in a modest emission reduction path. 


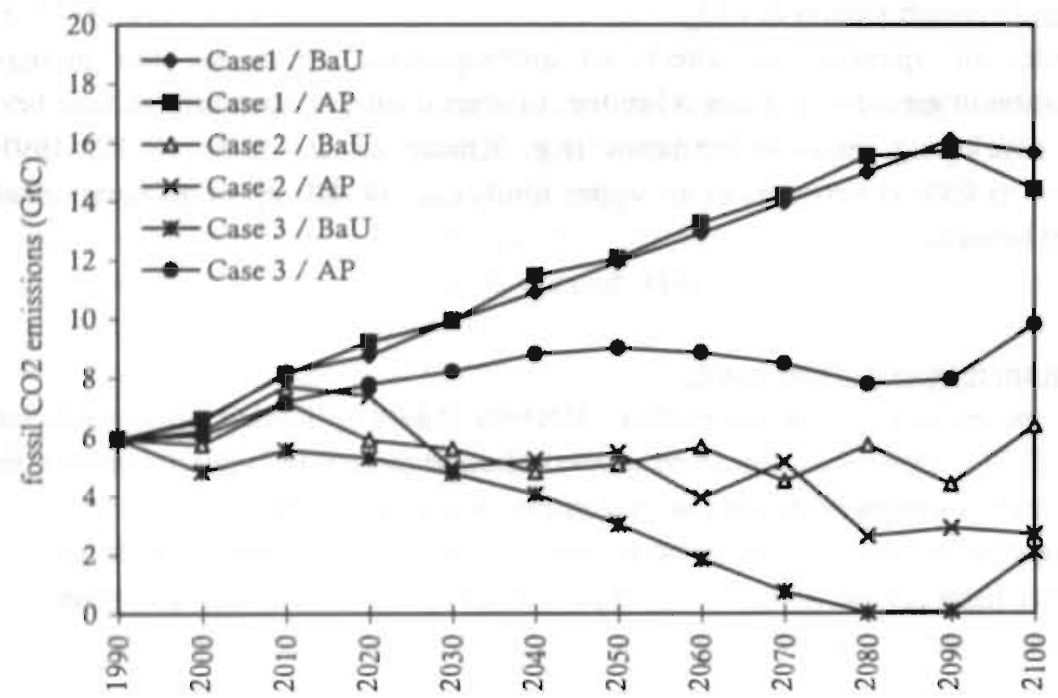

Figure 4.10: Optimal emission paths of fossil $\mathrm{CO}_{2}$ using OMEGA.

\section{Concentration of $\mathrm{CO}_{2}$}

If environmental constraints are not explicitly taken into account (Case 1), $\mathrm{CO}_{2}$ concentration will reach a level of $630 \mathrm{ppmv}$ by 2100 (Figure 4.11). A phase-out of fossil $\mathrm{CO}_{2}$ emissions (case 3 including $\mathrm{BaU}$ scenarios for non-fossil $\mathrm{CO}_{2}$ emissions) is the only strategy which leads to a decrease in the $\mathrm{CO}_{2}$ concentration. 


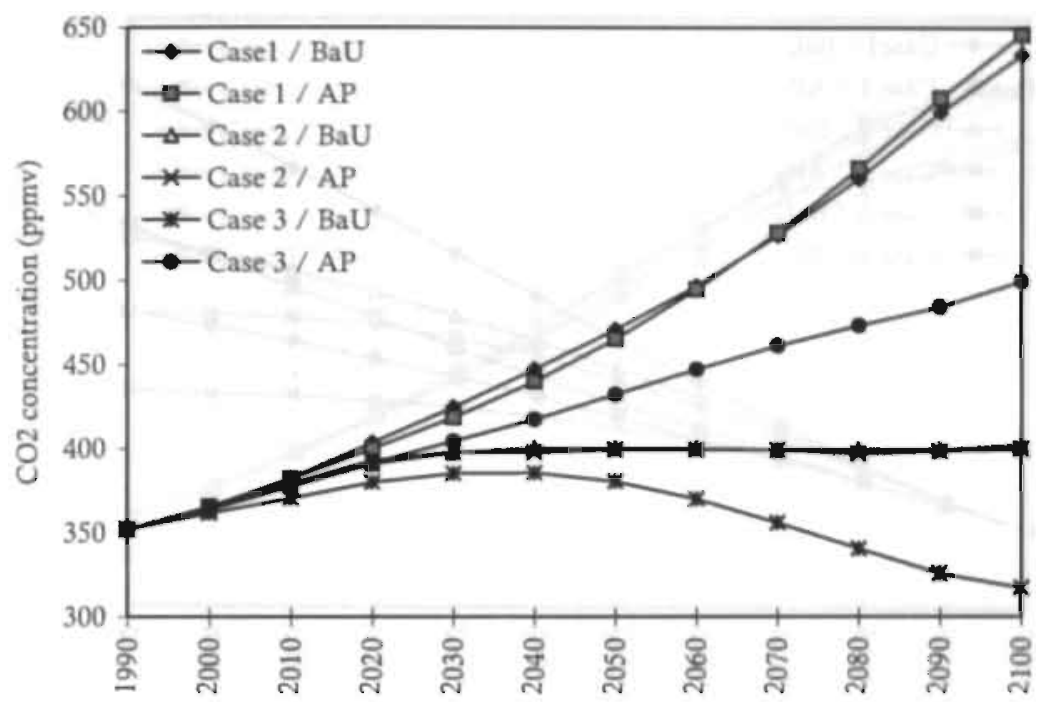

Figure 4.11: $\mathrm{CO}_{2}$ concentrations pertaining to optimal strategies according to OMEGA.

\section{Temperature Increase}

The extra temperature increase associated with the case in which not all greenhouse gases are controlled leads to an additional $1{ }^{\circ} \mathrm{C}$ temperature increase (Figure 4.12). Therefore, a cost-benefit policy leads to a temperature increase of between 2.6 and $3.5^{\circ} \mathrm{C}$, while meeting the concentration target results in a range of 1.5 to $2.5{ }^{\circ} \mathrm{C}$. The reader will note that temperature increases are significant in the next century in all cases, which may lead to serious impaçts on economic, agricultural, and social developments. 


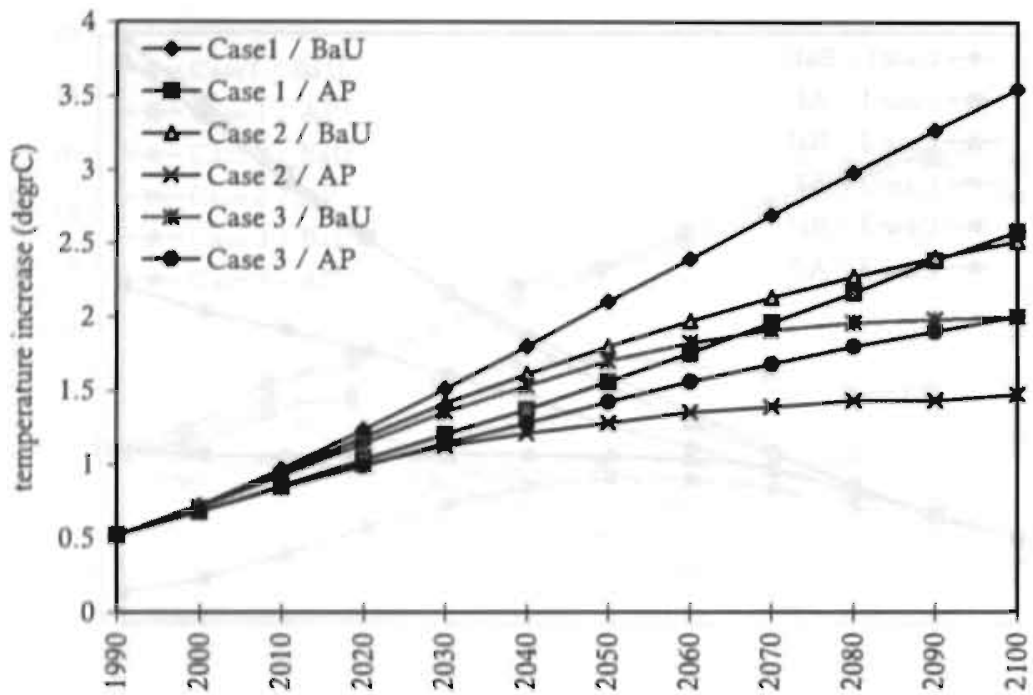

Figure 4.12: Temperature increase according to OMEGA.

\section{Output}

The differences in economic output among the various cases are relatively small throughout the next century (Figure 4.13). The output will at most, in case of a phaseout of fossil $\mathrm{CO}_{2}$ emissions, lead to a $12 \%$ lower output than in the optimal costbenefit case. Note that the economic output here is "green" in that it takes account of environmental impacts of economic activities in terms of market damage. However, non-market damage is not taken into account in this study. 


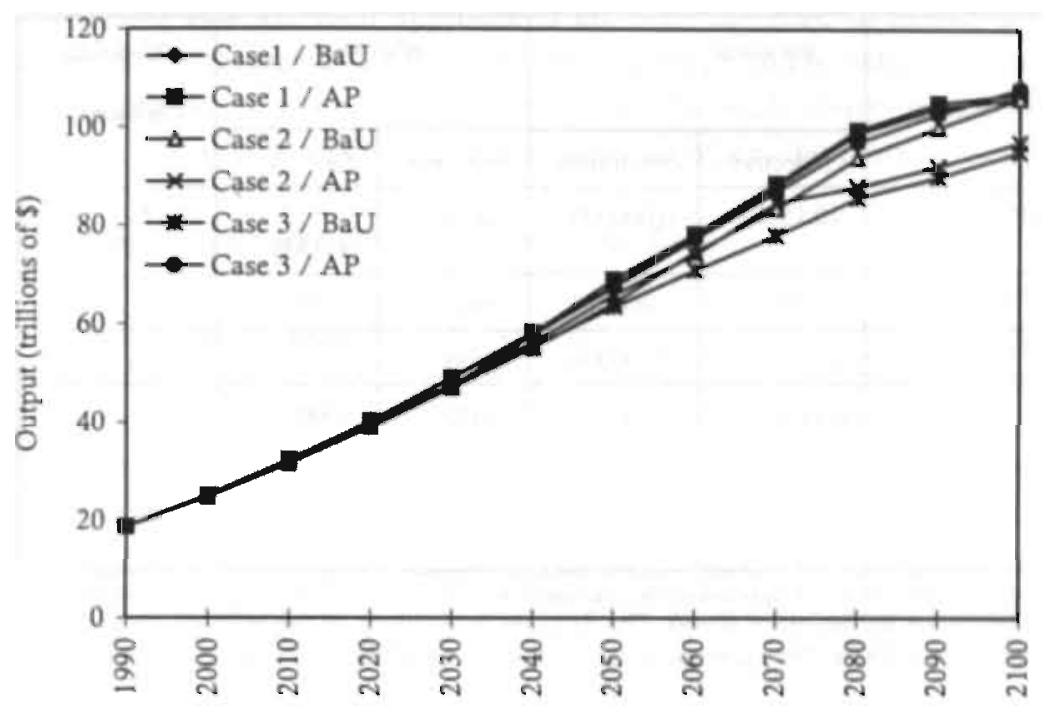

Figure 4.13: Economic output according to OMEGA.

\subsubsection{Performance Results}

The performance of the optimization approaches is illustrated for the solutions of the three test problems, starting the search at three starting points. In order to quantify the performance, criteria are distinguished, based on Hock and Schittkowski (1983):

\begin{tabular}{|l|l|}
\hline Accuracy & \\
\hline $\begin{array}{l}\text { objective: } \\
\text { constraints: }\end{array}$ & $\begin{array}{l}\text { percentage of difference with the best solution found } \\
\text { sum of violation of constraints ( } \mathrm{g}(\mathrm{u}) \text { ) }\end{array}$ \\
\hline Efficiency & \\
\hline $\begin{array}{l}\text { execution time (seconds): } \\
\text { function evaluations: }\end{array}$ & $\begin{array}{l}\text { cpu time on Silicon Graphics (Indy) workstation } \\
\text { number of model runs, }\end{array}$ \\
\hline reliability & \\
\hline failures (\%): & percentage of runs where no feasible solution was found \\
\hline
\end{tabular}

\section{Table 4.3: Performance criteria}

Detailed performance scores can be found in Appendix 4.2 and 4.3, while the average scores are shown in Table 4.4. 


\begin{tabular}{|c|c|c|c|c|c|}
\hline & \multicolumn{2}{|l|}{ accuracy } & \multicolumn{2}{|c|}{ efficiency } & \multirow{2}{*}{$\begin{array}{l}\text { reliability } \\
\% \text { failures }\end{array}$} \\
\hline & objective & constraints & run time & f.e. & \\
\hline SRSP & $10.15 \%$ & 0.000000 & 4079 & $\begin{array}{c}5 \\
(41152)\end{array}$ & $17 \%$ \\
\hline Penalty Method & $0.01 \%$ & 0.000014 & 26807 & 7305 & $0 \%$ \\
\hline SQP & $0.82 \%$ & 0.000000 & 8699 & 2097 & $75 \%$ \\
\hline GA & $\begin{array}{l}0.11 \% \\
0.10 \% \\
0.10 \% \\
0.10 \% \\
0.10 \%\end{array}$ & $\begin{array}{l}0.33 \\
0.32 \\
0.28 \\
0.26 \\
0.20\end{array}$ & $\begin{array}{c}3408 \\
6818 \\
10227 \\
13635 \\
17044\end{array}$ & $\begin{array}{l}1000 \\
2000 \\
3000 \\
4000 \\
5000\end{array}$ & $\begin{array}{l}0 \% \\
0 \% \\
0 \% \\
0 \% \\
0 \%\end{array}$ \\
\hline
\end{tabular}

Table 4.4: Performance of the 4 optimization approaches on the test problems. (Only feasible solutions are used to determine the accuracy score). The function evaluations of the reduced system are given between parentheses (SRSP). The experiments are performed on a Silicon Graphics Indy workstation.

SRSP has a very good performance on the unconstrained problem, where it found a solution close to the best solution found in a run time which is many times shorter then other used optimization techniques. However, SRSP fails on the constrained test cases where the efficiency is high, but the derived solutions are of a poor quality. Due to the fact that the dynamics of the constraints might not be taken into full account in the reduced version, SRSP was not able to find good solutions to the problem.

The penalty method which uses the original model, thus being able to cope with all of the dynamics inherent in the problem, was found to be a reliable and accurate, but time-consuming, approach for solving the large scale non-linear optimization. problem. Sequential Quadratic Programming is unreliable for the kind of problems as considered in this study as a result of the high degree of non-linearity which is not approximated within a quadratic framework. Therefore, quadratic subproblems do not help the search for the optimal solution, but lead to the rapid exhaustion of the search algorithm. The Genetic Algorithm performs very well on the unconstrained problem, but fails on the constrained ones. It is generally noted that genetic algorithms have problems in dealing, with constraints (Forest, 1993).

To summarize, additional research is necessary to overcome the difficulties of SRSP for the constraint optimization problems, in order to derive an efficient: optimization tool for large dynamic systems. In Chapter 6, I will use a genetic algorithm to perform the optimization experiments. The reason for this is that algorithms like the penalty method, SRSP and SQP perform relative nicely for sound 
mathematical systems; their approach is not been designed to handle a large scale simulation model (such as TARGETS, see Chapters 5 and 6), which consists of many discontinuities and non differentiable points. In such noisy search spaces, the robustness of genetical algorithms outperforms the other algorithms.

\subsubsection{Comparing Results of OMEGA with DICE}

The optimization problem was rendered a large-scale problem by replacing the small climate model of DICE by the 155 dimensional mathematical system which features in the IMAGE 1.0 model. I recognize that within OMEGA, there is a disbalance between the economic part and the environmental part. However, since the aim of the exercise is to show the impact of the choice of the modelling approach for the climate system on the "optimal" response strategies, I am, nevertheless, able to discuss the differences which occur as a result of using the "original" DICE model visà-vis the derived OMEGA model.

In order to compare the resuits, I propose to start by referring to identical "business-as-usual" results for both models, to which end I slightly adapt the DICE model. Originally, the temperature increase in 2100 for the DICE model was 3.3 degrees (Nordhaus, 1992;1993;1994). If I use the business-as-usual scenario borrowed from IMAGE 1.0 for the other trace gases $(\mathrm{O}(\mathrm{t})$ equation 4.7$)$, and if $\mathrm{I}$ only wish to control fossil $\mathrm{CO}_{2}$ emissions, and, furthermore, assume exogenous levels of CFC emissions and $\mathrm{CO}_{2}$ emissions due to land use changes, the temperature increase in 2100 is calculated as $3.8^{\circ} \mathrm{C}$.

The cost-benefit case solution does not significantly differ from the solution produced by OMEGA (Figure 4.14-4.17). OMEGA results support the case for a somewhat enhanced emission reduction strategy compared with DICE results. However, in order to meet the concentration target, emissions have to be stabilized at the level of $1 \mathrm{GtC}$, which is $80 \%$ lower than the level envisaged by OMEGA. The temperature target will be exceeded, unless other greenhouse gases are also controlled.

The differences in emission budgets for the next century can be read off from Table 4.5. The inclusion of environmental constraints leads to larger reductions in emissions according to DICE as opposed to OMEGA. The climate model incorporated in DICE and especially as expressed in equation (4.7) (the atmospheric concentration of $\mathrm{CO}_{2}$ ) is based on historical trends. Price (1995) argues that "representing $\mathrm{CO}_{2}$ uptake as instant disappearance, followed by a simple exponential uptake process, is too unrepresentative of what is known about oceanic and other sinks to give secure 
physical predictions". Furthermore, Price (1995) concludes that "gross analysis of historical trends in $\mathrm{CO}_{2}$ emissions and concentrations is likely to be misleading".

\begin{tabular}{|l|ll|ll|}
\hline Case Model & AP & $\begin{array}{c}\text { DICE } \\
\text { BaU }\end{array}$ & AP & $\begin{array}{l}\text { OMEGA } \\
\text { BaU }\end{array}$ \\
\hline Cost benefit & 1300 & 1285 & 1280 & 1245 \\
\hline Concentration target & 239 & 239 & 598 & 612 \\
\hline Temperature target & 529 & - & 888 & 338 \\
\hline
\end{tabular}

Table 4.5: Emission budgets of fossil $\mathrm{CO}_{2}$ for the period of 1990 to $2100 \mathrm{in} \mathrm{GtC}$, for $\mathrm{BaU}$ as well as for AP policy for other greenhouse gas emissions.

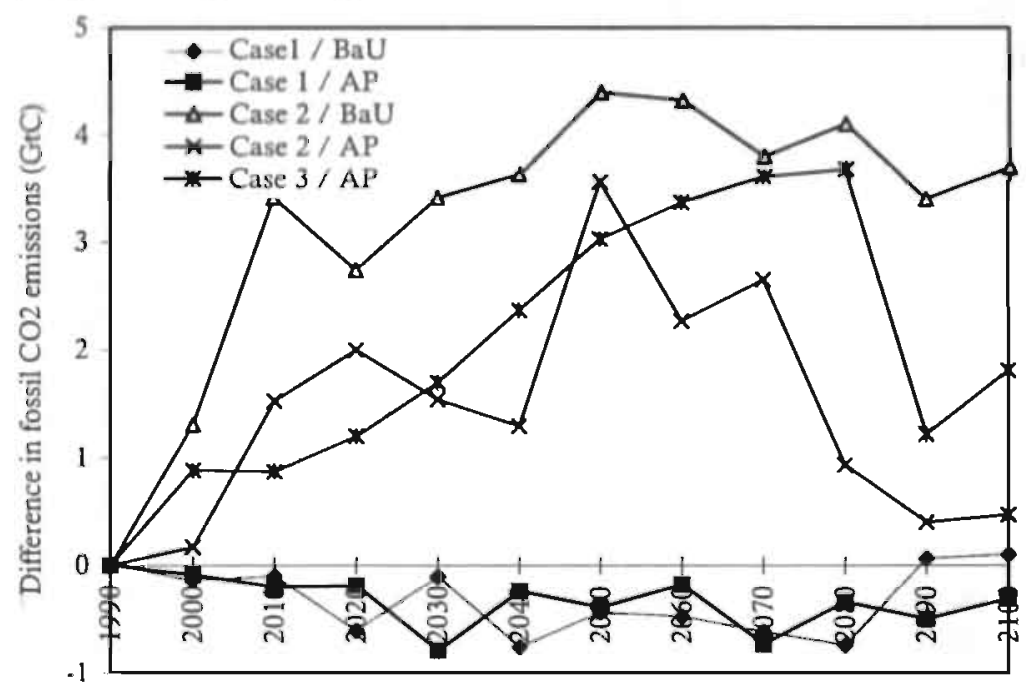

Figure 4.14: Difference in optimal emission paths between OMEGA and DICE. 


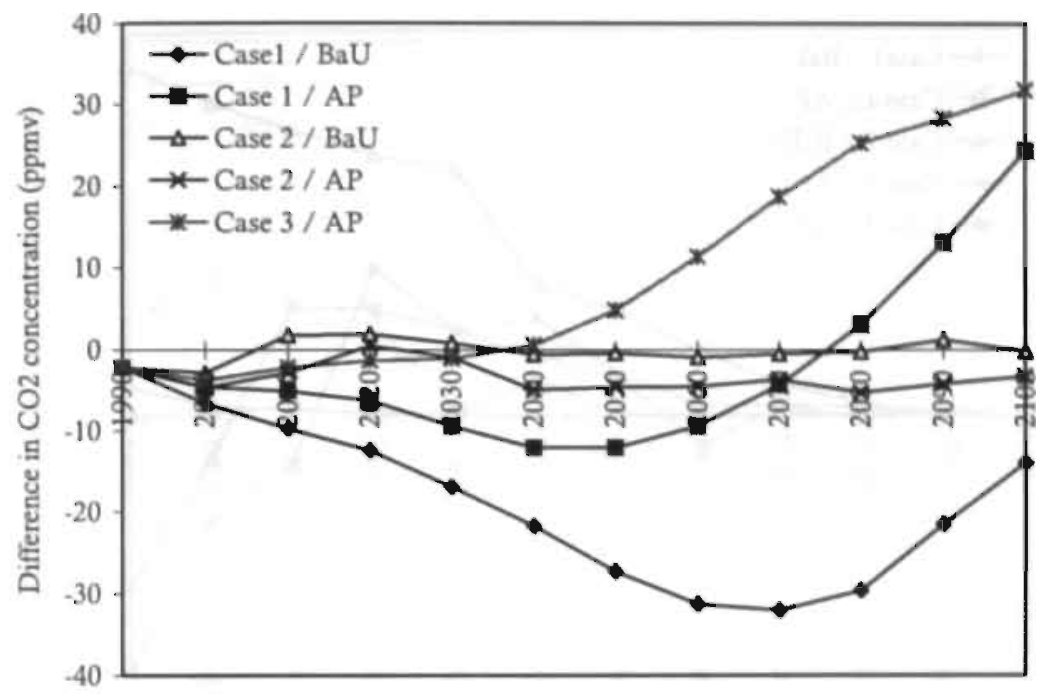

Figure 4.15: Difference in $\mathrm{CO}_{2}$ concentration between OMEGA and DICE.

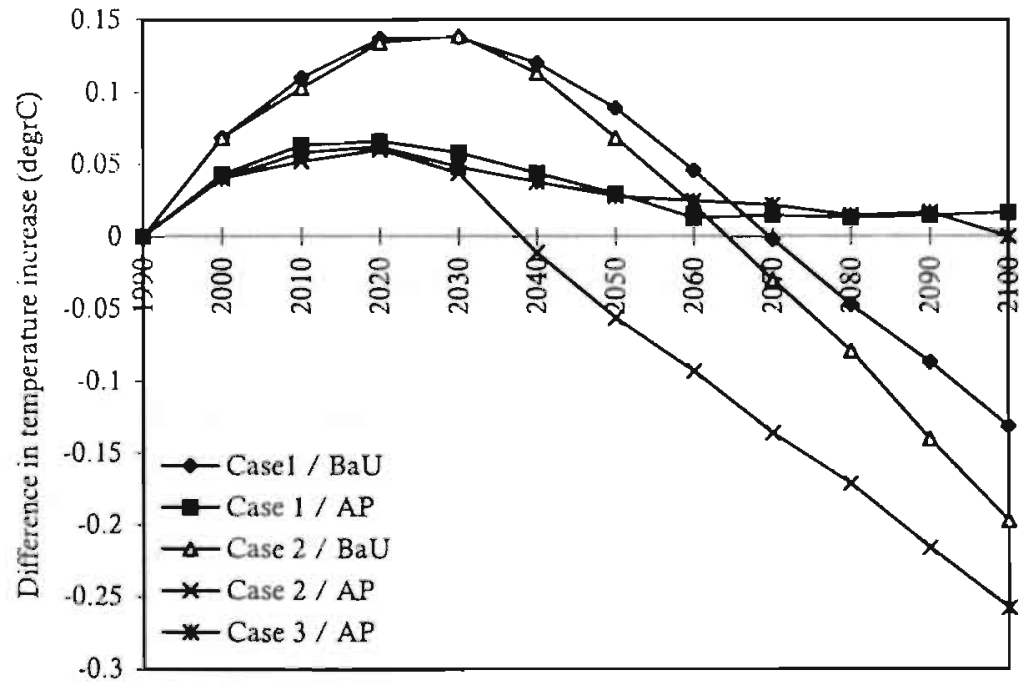

Figure 4.16: Difference in temperature increase betweer. OMEGA and DICE. 


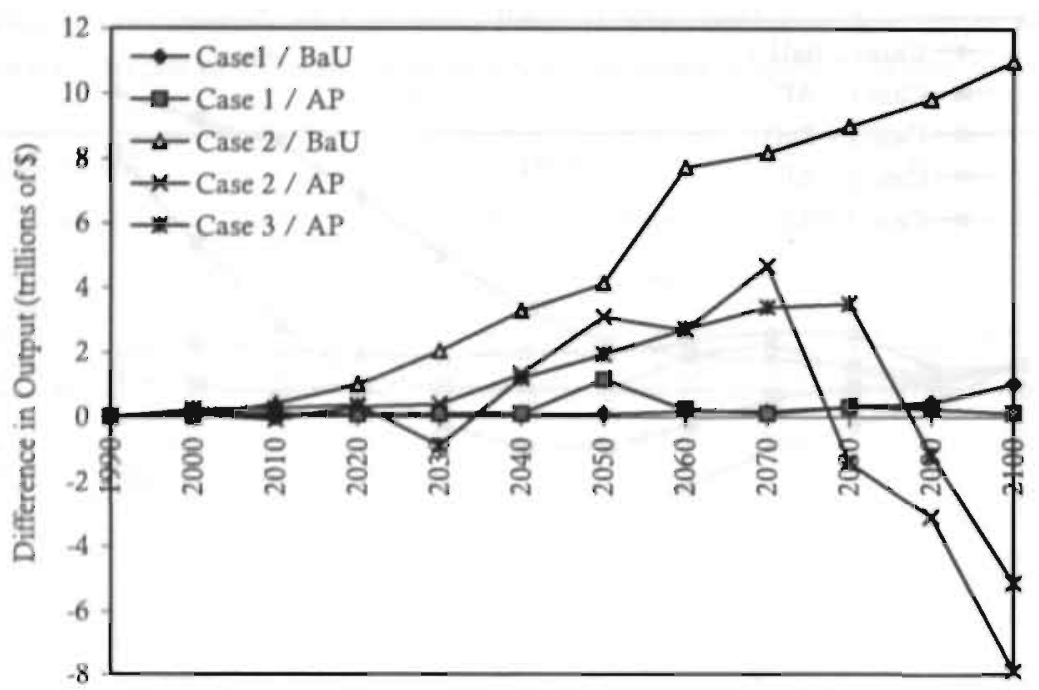

Figure 4.17: Difference in economic output between OMEGA and DICE.

The DICE model is, therefore, only able to estimate future concentrations by extrapolating historical behavior. If, however, in the next century, environmental policies were to be implemented which would result in a transformation of economic activities resulting in different patterns of fossil $\mathrm{CO}_{2}$ emissions, the DICE model would no longer be able to capture the consequences of such changes. OMEGA is designed to describe the climate processes insofar as they are understood by natural scientists, and is, therefore, able to capture a wider range of future scenarios. Important aspects which are missing in DICE are the fact that the ocean takes up less, the mare $\mathrm{CO}_{2}$ it has absorbed in the past, and the fact that the terrestrial biosphere takes up more $\mathrm{CO}_{2}$ if the $\mathrm{CO}_{2}$ concentration is high (fertilization effect). I have estimated $B$ and $\delta_{m}$ again based on results of OMEGA, where $\beta$ is kept constant on the original values if $\delta_{\mathrm{m}}$ is estimated, and the other way round. Figure 4.18 and 4.19 show the re-estimated parameter values $\beta$ and $\delta_{n}$ in time for the cost-benefit scenario and the $400 \mathrm{ppmv}$ scenario. The Figures illustrate that the parameters which are constant in DICE, but modelled in OMEGA, are not constant in time and differ between scenarios according to experiments with OMEGA. This is caused by the complex dynamics of the carbon cycles, exchanging carbon between oceans, atmosphere and terrestrial biosphere. 


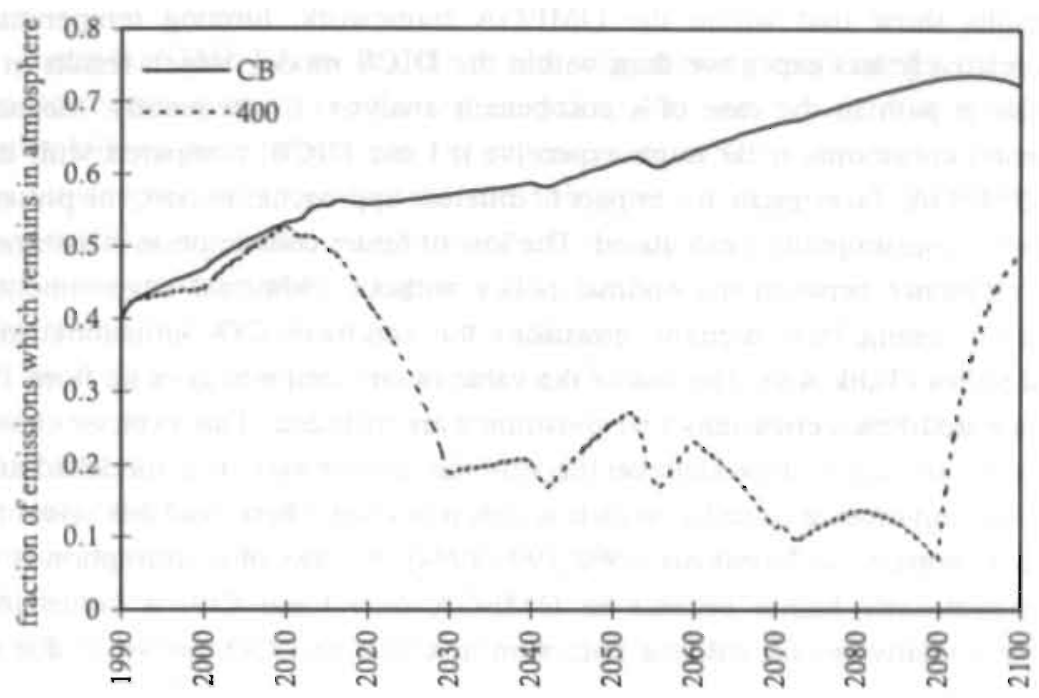

Figure 4.18: Estimated values of $\beta$ given a contrant $\delta$ and concentration estirnates from OMEGA for Cost-Benefit and 400 ppmv case.

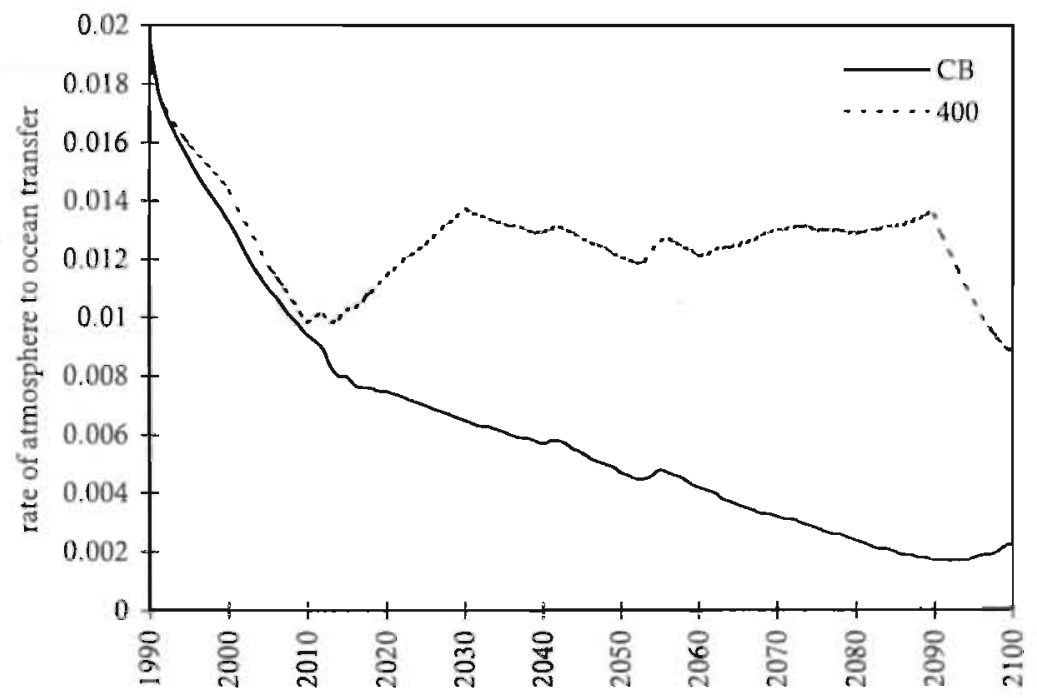

Figure 4.19: Estimated values of $\delta$ given a constant $\beta$ and concentration estimated from OMEGA for Cost-Benefit and 400 ppmv case. 
The results show that within the OMEGA framework, limiting temperature change is relatively less expensive than within the DICE model, which results in a lower emission path in the case of a cost-benefit analysis. Consequently, meeting environmental constraints is far more expensive if I use DICE, compared with the results of OMEGA. To estimate the impact of different approaches in cost, the present value of future consumption is calculated. The loss of future consumption is assumed to be the difference between the optimal policy without additional environmental constraints (assuming $\mathrm{BaU}$ scenario emissions for non-fossil $\mathrm{CO}_{2}$ emissions) and considered policy (Table 4.6). The loss of the value of consumption goes up from $1 \%$ to $6 \%$ in case additional environmental constraints are included. This exercise shows that the results are highly dependent on the way the climate system is modelled and underlines the importance of using models which rely on the best available scientific knowledge. Compared to Nordhaus $(1992 ; 1993 ; 1994)$, the loss of consumption is in all cases significantly higher because in OMEGA only fossil $\mathrm{CO}_{2}$ is controlled, neglecting the relatively cost-efficient reduction in $\mathrm{CFCs}$ and $\mathrm{CO}_{2}$ emission due to land use changes.

\begin{tabular}{|l|ccc|cc|}
\hline Case & \multicolumn{3}{|c|}{ DICE } & \multicolumn{2}{c|}{$\begin{array}{l}\text { OMEGA } \\
\text { BaU }\end{array}$} \\
\hline Cost benefit & AP & BaU & AP & Ba \\
& -2.6 & 0.0 & -2.8 & 0.0 \\
& $(0.2 \%)$ & $(0.0 \%)$ & $(-0.3 \%)$ & $(0.0 \%)$ \\
\hline Concentration target & 41.2 & 66.4 & 19.0 & 20.7 \\
& $(3.8 \%)$ & $(6.2 \%)$ & $(1.8 \%)$ & $(1.9 \%)$ \\
\hline Temperature target & 12.8 & & 1.6 & 43.6 \\
& $(1.2 \%)$ & & $(0.1 \%)$ & $(4.1 \%)$ \\
\hline
\end{tabular}

Table 4.6: Loss of discounted value of consumption compared to the reference case (cost-benefit assuming $\mathrm{AP}$ scenarios for non-fossil $\mathrm{CO}_{2}$ emissions) (in trillions of 1989 US \$). The loss of consumption as percentage of the discounted value of consumption of the reference case is given. between parentheses.

\section{Delayed Response}

Suppose a reduction policy according to the cost-benefit case is started. After a number of years, a new target is set, for example, because of new scientific insights on consequences of climate change. Suppose that such a new target is a $\mathrm{CO}_{2}$ concentration stabilization on a level of 400 ppmv. A number of experiments have been constructed in which the starting year of implementing additional mitigation is varied. The starting years as used in this analysis are 1990, 2000, 2010 and 2020. 
Using the OMEGA model, the results suggest that a delay until 2010 may lead to drastic reduction in order to avoid exceeding 400 ppmv (Figures 4.20 and 4.22). If policy follows the reference cost-benefit case until 2030, the concentration will exceed the target level for about two decades. However, a delay until 2000 will not have severe effects on future emission reductions. In case DICE is used, the required reductions are more drastic (Figure 4.21), and the $\mathrm{CO}_{2}$ concentration level exceeds the $400 \mathrm{ppmb}$ concentration targets, if the enhanced mitigation policy is not implemented before 2020 (Figure 4.23). The ignorance of changing carbon flows within the carbon cycle due to a human disturbance will lead to more drastic and costly reductions, according to DICE than according to results with OMEGA.

Note that the results are the consequence of differences between modelling the climate system. I did not vary, for example, economic and technical assumption which may also change the insights in suitable response policies. These results are, therefore, no advocate for a wait-and-see policy. They do illustrate a reason why a model like DICE leads to advice of modest emission reductions: it costs too much emission reductions to slow down the $\mathrm{CO}_{2}$ concentration increase. 


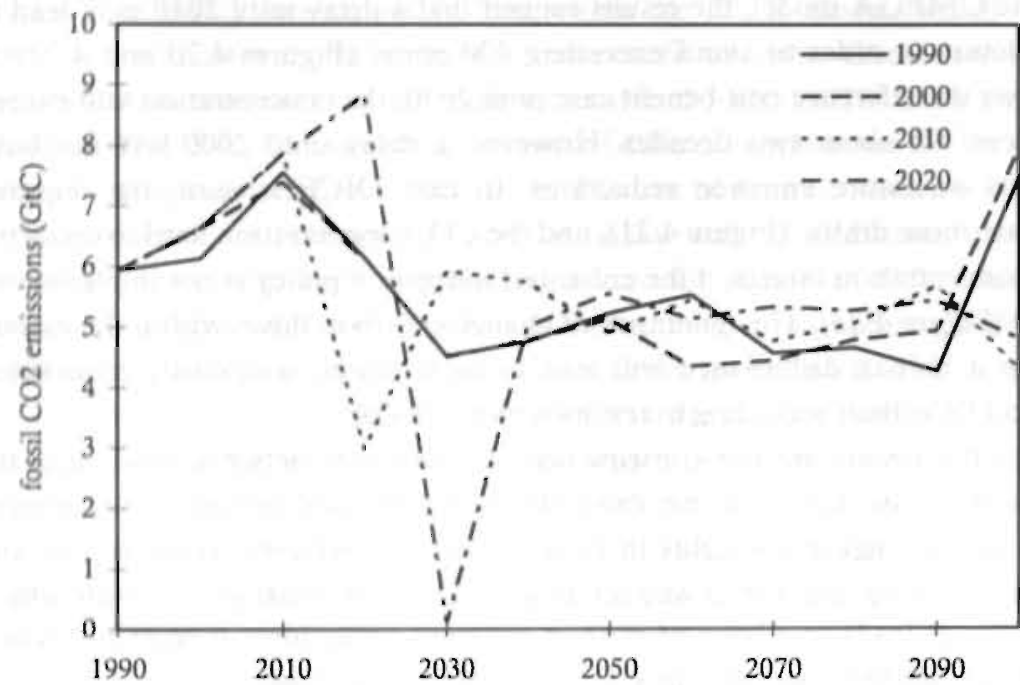

Figure 4.20: Fossil $\mathrm{CO}_{2}$ emissions for delayed response strategies starting in 1990, 2000, 2010 and 2020 using the OMEGA model meeting the 400 ppmv target.

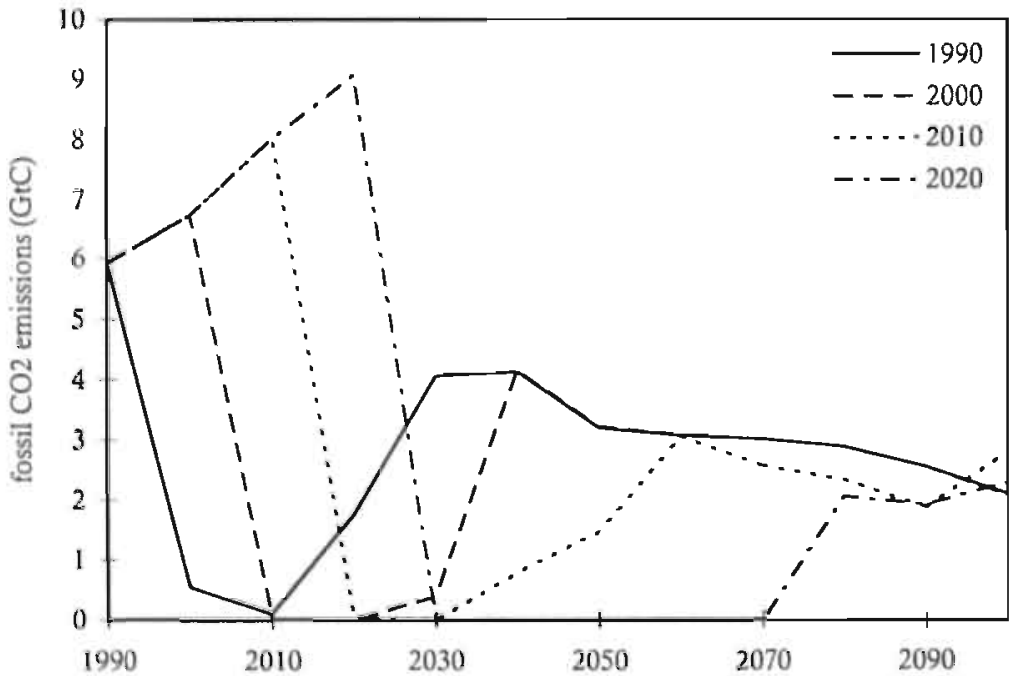

Figure 4.21: Fossil $\mathrm{CO}_{2}$ emissions for delayed response strategies starting in 1990, 2000, 2010 and 2020 using the DICE model meeting the 400 ppmv target. 


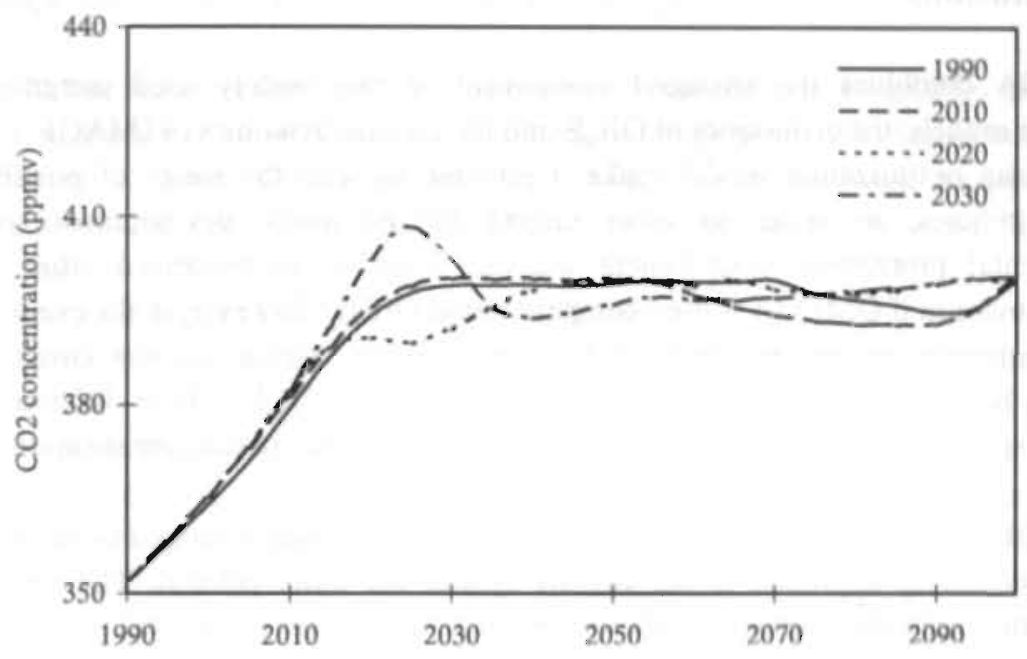

Figure 4.22: $\mathrm{CO}_{2}$ concentration for delayed response strategies for experiments with OMEGA .

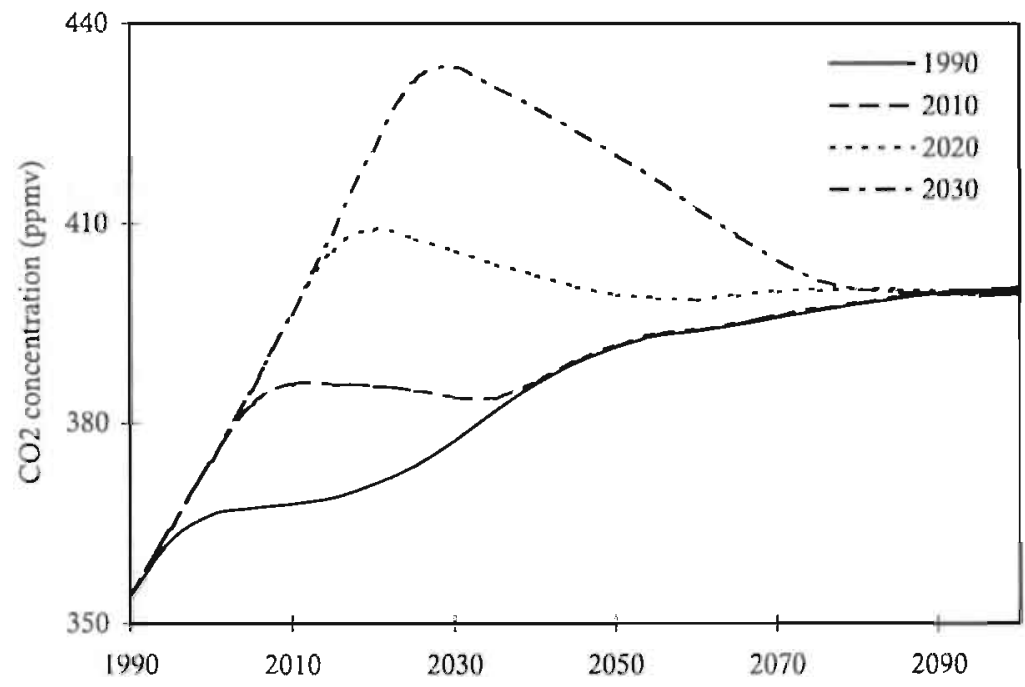

Figure 4.23: $\mathrm{CO}_{2}$ concentration for delayed response strategies for experiments with DICE. 


\subsection{Conclusions}

OMEGA combines the strongest component of two widely used integrated assessment models: the economics of DICE and the climate dynamics of IMAGE 1.0. The resulting optimization model make it possible to scan the range of possible response policies, in order to meet targets for economic development and environmental protection. Cost benefit analysis leads to an enhanced effort in reducing emission if OMEGA is used compared with DICE. However, in the event of severe constraints on concentration levels or temperature change, far less emission reductions have to be made, if I use OMEGA instead of DICE. These differences could be explained by the different descriptions of the dynamics of the climate system.

Rather than simply providing a sensitivity analysis, the insights gained by using a different modelling approach to the climate system are more general. The results underline the conceptual limitation of most optimization studies in the field of climate change by ignoring the dynamies of the climate system as reasonably understood by natural science.

Optimization approaches which use elegant, transparent descriptions of the climate system cannot deliver adequate images of the underlying complex systems' processes and dynamics and, thus, lead to results which may be misleading, given the state-of-the-art knowledge. While I recognize the potential richness of applying optimization techniques to the climate change problem, I argue that such an approach needs to use a description of the climate system as is momentarily understood in the light of current knowledge derived from the natural scientific community.

The critical notes made in this Chapter are meant to contribute to the development of more sophisticated tools for scanning climate change response strategies. In the following Chapters, such an attempt is made by applying optimization on the integrated assessment model for global change TARGETS. 


\section{Appendix 1: Reduced Version of OMEGA}

Before reducing the model, it is important to consider which variables are essential. It is necessary to know is how the global mean temperature will change in response to changes in fossil $\mathrm{CO}_{2}$ emission. This leads to the following variables in the mathematical system of IMAGE 1.0, which represents the reduced IMAGE 1.0 version:

- concentration of $\mathrm{CO}_{2}, \mathrm{x}_{13}(\mathrm{t})$ from IMAGE 1.0.

- change in temperature in the mixed surface layer of the ocean, $x_{141}$ from IMAGE 1.0 .

- change in the global mean surface temperature $x_{158}(t)$.

The rest of the model is discarded. The combined model is, therefore, reduced from 163 equations to a system of 9 equations, which can be solved by Runge-Kutta method 2 with a fixed time step of 0.5 .

$$
\begin{aligned}
& \operatorname{maximize} \sum_{t=1990}^{2100} l(t) \cdot \ln \left(y_{2}(t)\right) \cdot(1+\rho)^{(1990-1)} \\
& z_{1}(t), z_{2}(t)
\end{aligned}
$$

(1) $y_{1}(t)=y_{9}(t) \cdot a(t) \cdot\left[y_{3}(t)\right]^{r} \cdot l(t)^{2 \cdot \gamma}$

(2) $y_{2}(t)=\frac{y_{t}(t)-z_{l}(t)}{l(t)}$

(3) $\frac{d y_{3}(t)}{d t}=z_{1}(t)-\delta_{k} \cdot y_{3}(t)$

(4) $y_{4}(t)=\left[1-z_{2}(t)\right] \cdot \sigma(t) \cdot y_{t}(t)$ 
(5) $\frac{d x_{13}(t)}{d t}=0.47 \cdot y_{d}(t)+\varphi(t)+\tau(t)$

(6) $x_{I S 7}(t)=\frac{\lambda^{*} \cdot \Delta T_{2 x c 0_{1}}}{\ln (2)} \cdot \ln \left(\frac{x_{13}(t)}{x_{13}(1900)}\right)+x_{I S 8}(t)$

(7) $\frac{d x_{111}(t)}{d t}=8 / 3 \cdot x_{92}(t) \cdot\left(\lambda / c_{m}+8 / 3\right) \cdot x_{141}(t)+x_{156}(t) / c_{m}$

(8) $x_{156}(t)=\frac{f \cdot x_{157}(t)+k \cdot x_{191}(t)}{f \cdot \lambda+k}$

(9) $y_{9}(t)=\frac{1-b_{1} \cdot z_{2}(t)^{b_{2}}}{1+\theta_{1} \cdot\left[x_{156}(t)\right]^{\theta_{2}}}$

where

$$
\begin{aligned}
\varphi(t) & =0.47 \cdot\left(\frac{x_{1}(t)}{\alpha_{1}}+\frac{x_{12}(t)}{\alpha_{12}}+\sum_{i=35}^{22} x_{i}(t) \cdot \alpha_{i}\right)-\alpha \cdot x_{13}(t) \\
\tau(t) & =N(X)_{13}(t)
\end{aligned}
$$

and where:

$$
\frac{d \sigma(t)}{d t}=\exp \left(\delta_{\sigma} \cdot t\right) \cdot \sigma(t) \quad \frac{d l(t)}{d t}=\exp \left(\delta_{l} \cdot t\right) \cdot l(t) \quad \frac{d a(t)}{d t}=\exp \left(\delta_{a} \cdot t\right) \cdot a(t)
$$

\footnotetext{
$\mathrm{a}_{\mathrm{i}} \quad$ are coefficients

$\mathrm{c}_{\mathrm{m}} \quad$ in the bulk heat capacity of the ocean mixed layer $\left(10.97 \mathrm{~W} \mathrm{y} / \mathrm{m}^{2}{ }^{\circ} \mathrm{C}\right)$ and where: $x_{1}, x_{12}, x_{35}, x_{36}, \ldots, x_{61}, x_{62}, x_{92}, x_{158}$ and $N(X)_{13}$ are exogenous input variables
} 
Appendix 2: Performance results for all experiments non- $\mathrm{CO} 2 \mathrm{BaU}$

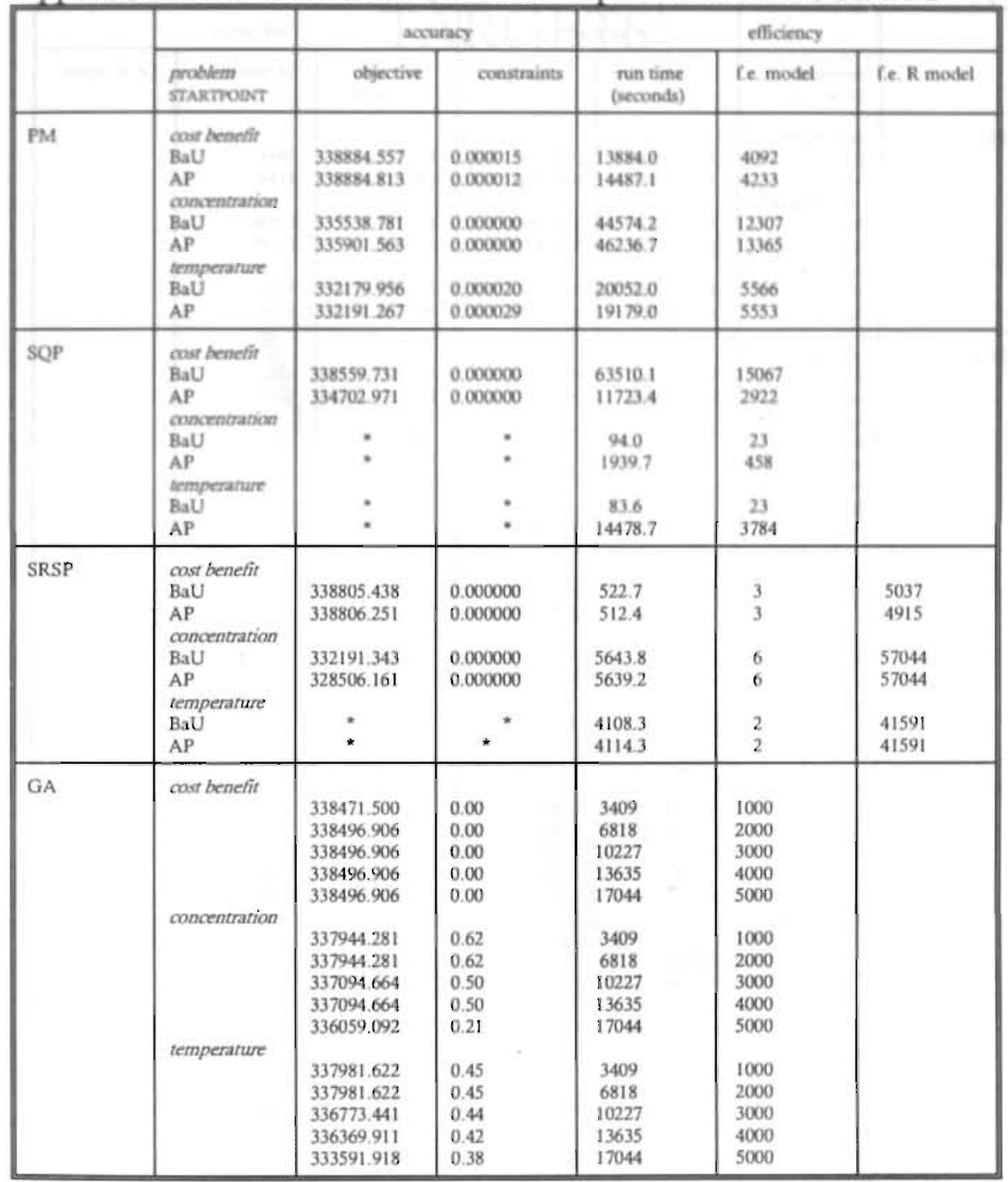

* no feasible solution found 
Appendix 3: Performance results for all experiments non- $\mathrm{CO} 2 \mathrm{AP}$

\begin{tabular}{|c|c|c|c|c|c|c|}
\hline & \multirow[b]{2}{*}{$\begin{array}{l}\text { problem } \\
\text { STARTrOnNT }\end{array}$} & \multicolumn{2}{|c|}{ accuracy } & \multicolumn{3}{|c|}{ efficiency } \\
\hline & & objective & constraints & $\begin{array}{l}\text { run time } \\
\text { (seconds) }\end{array}$ & fe. model & Ce. $\mathrm{R}$ mode \\
\hline PM & $\begin{array}{l}\text { cost benefit } \\
\mathrm{BaU} \\
\mathrm{AP} \\
\text { concentration } \\
\mathrm{BaU} \\
\mathrm{AP} \\
\text { temperature } \\
\mathrm{BaU} \\
\mathrm{AP}\end{array}$ & $\begin{array}{l}339293.318 \\
339292.969 \\
\\
336171.313 \\
336188.938 \\
\\
338689.000 \\
338699.563\end{array}$ & $\begin{array}{l}0.000096 \\
0.000000 \\
0.000000 \\
0.000000 \\
\\
0.000000 \\
0.000000\end{array}$ & $\begin{array}{l}13292.8 \\
14408.9 \\
\\
45775.3 \\
46378.0 \\
15378.1 \\
28037.2\end{array}$ & $\begin{array}{l}3894 \\
4149 \\
\\
12530 \\
13188 \\
\\
4266 \\
4518\end{array}$ & \\
\hline SQP & $\begin{array}{l}\text { eost benefit } \\
\text { BaU } \\
\text { AP } \\
\text { concentration } \\
\text { BaU } \\
\text { AP } \\
\text { temperature } \\
\text { BaU } \\
\text { AP }\end{array}$ & $\begin{array}{c}338792.842 \\
335083.383 \\
: \\
\dot{3} \\
\dot{334092.243}\end{array}$ & $\begin{array}{r}0.000000 \\
0.000000 \\
\vdots \\
\vdots\end{array}$ & $\begin{array}{c}9777.0 \\
1102.5 \\
\\
84.9 \\
1243.1 \\
\\
169.2 \\
182.4\end{array}$ & $\begin{array}{l}2242 \\
261 \\
\\
23 \\
2851 \\
\\
46 \\
26\end{array}$ & \\
\hline SRSP & $\begin{array}{l}\text { cosi benefit } \\
\mathrm{BaU} \\
\mathrm{AP} \\
\text { concentration } \\
\mathrm{BaU} \\
\mathrm{AP} \\
\text { temperarure } \\
\mathrm{BaU} \\
\mathrm{AP}\end{array}$ & $\begin{array}{l}339232.344 \\
33923 ! .000 \\
\\
328506.161 \\
328506.647 \\
\\
331371.439 \\
331370.433\end{array}$ & $\begin{array}{l}0.000000 \\
0.000000 \\
\\
0.000000 \\
0.000000 \\
0.000000 \\
0.000000\end{array}$ & $\begin{array}{l}539.2 \\
560.1 \\
10753.2 \\
10736.6 \\
\\
2910.8 \\
2909.3\end{array}$ & $\begin{array}{l}3 \\
3 \\
\\
4 \\
4 \\
11 \\
11\end{array}$ & $\begin{array}{l}5169 \\
5344 \\
\\
109199 \\
109198 \\
\\
28847 \\
28847\end{array}$ \\
\hline GA & $\begin{array}{l}\text { coss benefit } \\
\text { concentration }\end{array}$ & $\begin{array}{l}338973.188 \\
338973.188 \\
338973.188 \\
338973.188 \\
338973.188 \\
\\
338005.536 \\
337100.973 \\
337656.536 \\
337656.536 \\
337656.536 \\
338047.191 \\
338047.191 \\
338199.443 \\
338088.912 \\
337179.756\end{array}$ & $\begin{array}{l}0.00 \\
0.00 \\
0.00 \\
0.00 \\
0.00 \\
\\
0.63 \\
0.55 \\
0.5 ! \\
0.51 \\
0.51 \\
\\
0.30 \\
0.30 \\
0.21 \\
0.11 \\
0.07\end{array}$ & $\begin{array}{c}3408 \\
6818 \\
10227 \\
13635 \\
17044 \\
\\
3408 \\
6818 \\
10227 \\
13635 \\
17044 \\
\\
3408 \\
6815 \\
10227 \\
13635 \\
17044\end{array}$ & $\begin{array}{l}1000 \\
2000 \\
3000 \\
4000 \\
5000 \\
\\
1000 \\
2000 \\
3000 \\
4000 \\
5000 \\
\\
1000 \\
2000 \\
3000 \\
4000 \\
5000\end{array}$ & \\
\hline
\end{tabular}

* no feasible solution found 


\section{5 \\ TARGETS ${ }^{1}$}

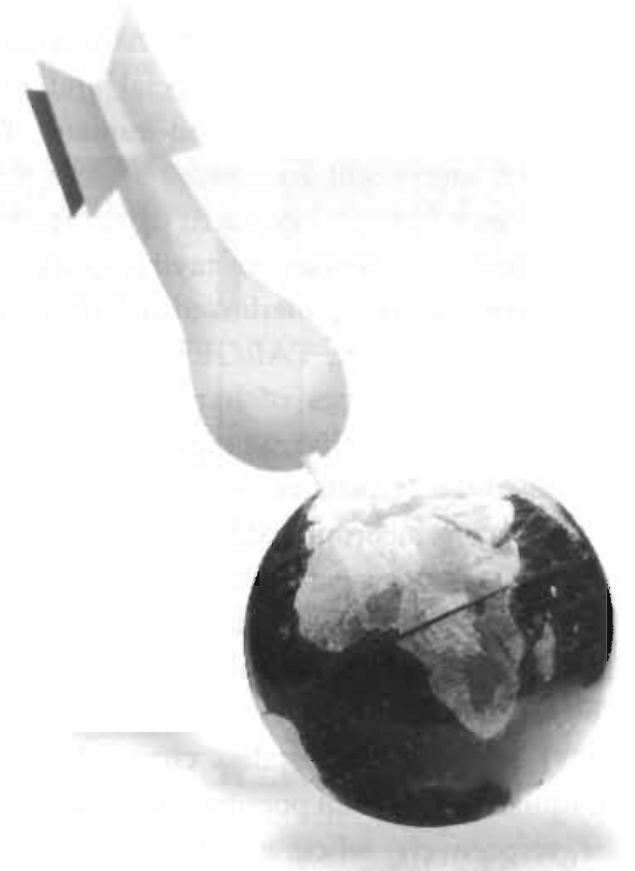

Various parts of this Chapter are based on Rotmans and De Vries (1996). 


\subsection{Introduction}

In this Chapter, the backgrounds and general structure of TARGETS (Tool to Assess Regional and Global Environmental and health Targets for Sustainability) are discussed, after which the various submodels are briefly described. In the next Chapter, the TARGETS model is used for optimization oriented experiments. An extensive description of the submodels and analysis with the whole model is presented in Rotmans and De Vries (1996).

TARGETS is intended to be used to perform an analysis and assessment on a global scale of the quantitative linkages among social and economic processes, biophysical processes, and effects on ecosystems and humans. The justification for developing such an integrated assessment framework is that it is among the first attempts to appraise global change from an integrated dynamic perspective. Based on the various subsystems of the whole system portraying global change, a series of highly-aggregated modules have been built, interlinked and ultimately integrated. This results in an overall integrated framework, TARGETS. The TARGETS framework enables the consequences of several types of human influences to be evaluated simultaneously. It is, hereby, envisaged that synergetic effects, which are currently beyond the horizon of predictive competence, may be brought into view. A twodimensional integration approach is incorporated in TARGETS in the following way. The TARGETS integrated framework basically consists of a population and health submodel, a energy/economy submodel, a biophysics submodel, a land/soil submodel, and a water submodel, which are all interlinked. All types of models comprise a linkage of pressure, state, impact, and response modules, in this way representing the (vertically) integrated cause-effect chain. All causative or pressure models describe the developments in human population and health, energy/economy, land, soil, and water use, respectively, where all developments are interlinked. In a similar way, all models describing the disturbed states of the underlying system, are coupled, just as all impact models are. By coupling the various causes, states, and impacts for the various subsystems underlying the submodels, the horizontal integration comes into play. This enables the representation of TARGETS as a twodimensional integration matrix, as shown conceptually in Figure 5.1. However, in order to keep the TARGETS framework as flexible as possible, it should comprise autonomously functioning submodels, i.e. each submodel can run independently of all other submodels. However, running a submodel of TARGETS as a stand-alone tool implies that the interlinkages with the other submodels are cut off, ignoring then a number of interactions and feedback mechanisms which may be crucial for the longterm global dynamics. On the other hand, having a stand-alone version of a submodel 
is useful, in particular for addressing submodel-specific issues and for the calibration and validation process.

First, the conceptual backgrounds of the TARGETS model will be discussed before the modelling framework will be discussed in more detail.

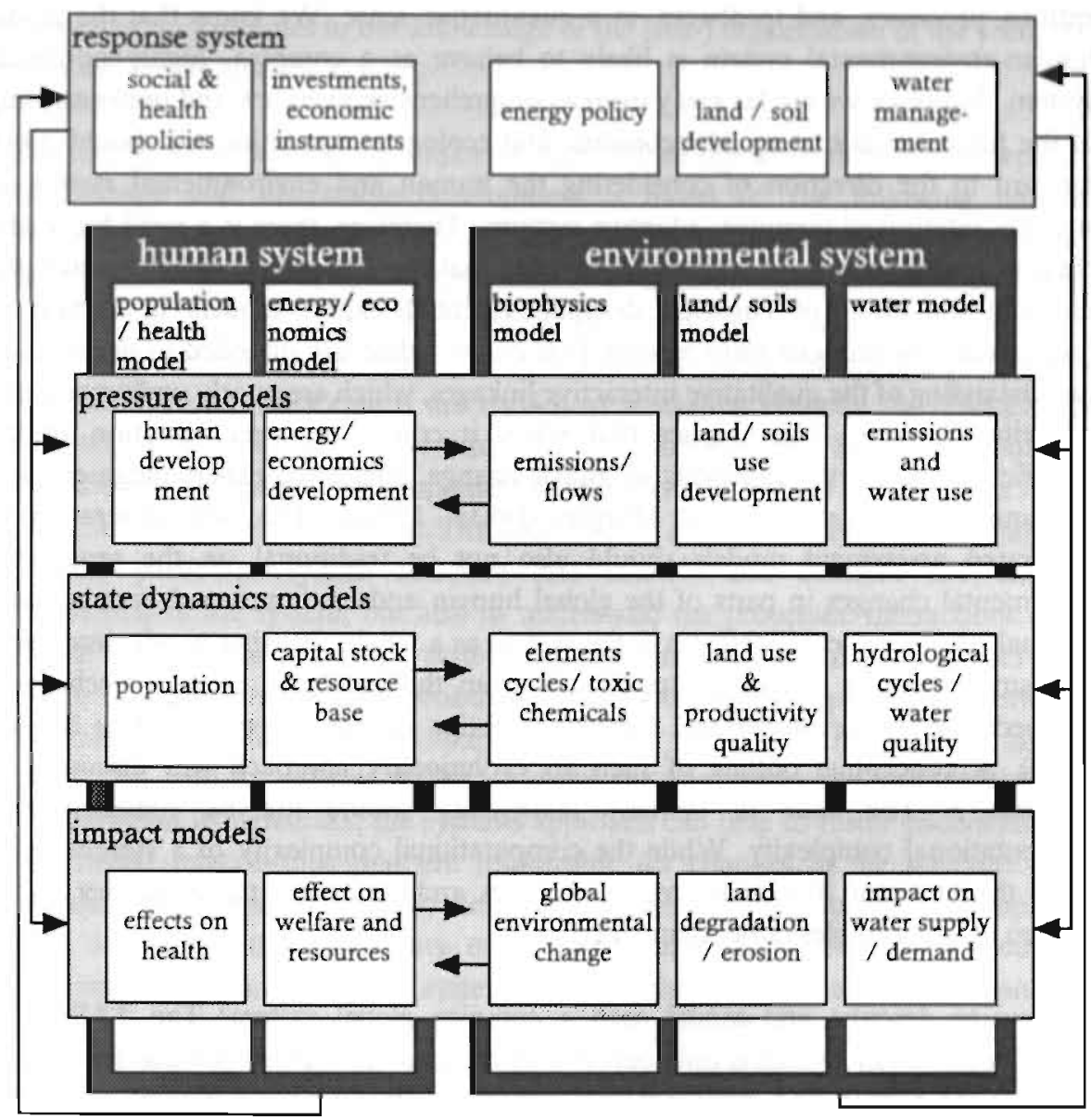

Figuur 5.1: Modular perspective of TARGETS, version 1.0 


\subsection{TARGETS Modelling Paradigm}

\subsubsection{The Integrated Systems Approach}

In light of the far from sufficient knowledge in natural and social sciences pertaining to global change, it is absolutely clear that it is impossible to model all entities, processes, and feedbacks, in a quantitative sense. We know that the global human-environmental system is likely to behave as a complex, highly nonlinear system. Although we are far away from a comprehensive overview and understanding of the fundamentals of social, economic and ecological dynamics, we should move forward to the direction of considering the human and environmental system as strongly interlinked complex, adaptive systems. Therefore, there is a need for a new class of global integrated assessment models that, on one hand, try to capture the essential dynamics of complex discipline-oriented expert models in meta-form (metamodels or reduced form model), but, on the other, are intended to promote an understanding of the qualitative interactive linkages, which are poorly understood in a quantitative sense. This implies that when it comes to implementation of the insufficiently understood aspects of global change, subjective expert judgment is a prerequisite (Dowlatabadi and Morgan, 1993a; 1993b). The new generation of integrated assessment models should also not be traditional, in the sense that incremental changes in parts of the global human and environmental system cause gradual and incremental changes in the system as a whole. In other words, integrated assessment models should gradually shift from the purely or pseudo mechanistic perspective to the evolutionary, complex adaptive systems' perspective (see Section 3.4.1). A conceptual outline of such an evolutionary approach was discussed in Chapter 3. The issue of system's complexity should not be confused with computational complexity. While the computational complexity of a system arises from the fact that it includes many variables and processes, the dynamics of the system may be simpler rather than complex.

How to describe and model such a complex global system? The TARGETS modelling strategy, which is only one way of proceeding, is the following: for specific parts of the human and environmental system, relatively simple models have been developed, with a transparent structure, but often complex dynamics. These relatively simple models (metamodels or reduced form models) are then linked to an overall framework, the TARGETS model, that can be analyzed. In this way, the TARGETS model is a composite framework of relatively simple systems - represented by metamodels-, which, as a whole, functions as a complex system. Although relatively 
simple in its individual components, the TARGETS model is quite complex as a whole, due to the many cause-effect relationships and feedbacks taken into account. Incremental changes in conditions of subsystems may then result in considerable change in the results of the overall system, which may not always be expected beforehand. Following this modelling approach, our knowledge of the global change phenomenon can be built up gradually, leading to improved understanding of the fundamental uncertainties in our knowledge of the (self-) organization of the complex human and environmental system.

The justification for adopting a systems approach to operationalize the concepts of global change and sustainable development is based on the following line of argument. Sustainable development is closely allied to the natural resilience and buffer capacity of the biosphere in relation to anthropogenic disturbances. The disturbance on a global scale is denoted as global change and can be represented by a set of interrelated cause-effect chains. To operationalize global change and sustainable development, cause-effect chains are chosen as a starting point in the TARGETS framework. The inextricably interconnected cause-effect chains form an organized whole, a complex system, the properties of which are more than just the sum of its constituent parts, the subsystems. The objective of systems analysis is not only to study the particular system structure and classify and describe the entities (components) of the system, but also to understand the processes, interactions, and feedback mechanisms within the system that generate changes in its dynamics and structure. It enables a synoptic approach that addresses the interdependencies between the cause-effect chains. Given. the complexity of the Earth system under consideration, and the relative ignorance about the basic processes and interactions that determined its dynamics, the systems approach can help to foster understanding of the causal relationships that are responsible for changes in the structure and dynamics of the system. Therefore, the systems approach seems to be an appropriate method to capture the complexity of the interrelationships between the various subsystems for the complex Earth system. A prerequisite for such a systems approach is inter- and multi-disciplinarity, based on the integration of knowledge gleaned from a variety of scientific disciplines. It then provides the opportunity to conceptualize the issues of global change and sustainable development in a holistic, integrated way. 


\subsubsection{Conceptual Framework}

Obviously, it is clear that building a single model capable of addressing all the nuances of the global change problem is not possible. The price for building any integrated model of the global change problem is that many issues by design have to be treated at a very high level of abstraction, abstractions which according to detractors of integrated assessment modelling, vitiate the value of any insights gained from their development and application. This concern is precisely why integrated assessment modelling efforts are increasingly focused on the systematic characterization of model weaknesses and strengths.

What can be done, however, is to present a relative simple, transparent and generic conceptual framework which can be used as a blue-print for a computer simulation model. The conceptual model can serve as a "thinking", organizing framework to analyze and structure the discussion concerning global change and to demonstrate the coherence and interrelationships between the various issues constituting global change. Application of the conceptual framework allows for the analysis of the dynamic linkages and trade-offs between social, economic, and environmental changes, based upon which projections for early warning purposes can be made. The framework should be capable of highlighting the most important relations between the societal (human) subsystem and the ecological (environmental) subsystem.

Any attempt, however, to represent the numerous interlinkages between society and the environment as a complex system is doomed to be incomplete and imperfect. Yet, even a simplified, but integrated conceptual model is thought to be more useful as a guide to global change and sustainable development than highly detailed models of subsystems that cover only some parts of the phenomenon.

The conceptual human-environmental framework is chosen to be as generic as possible, i.e. not tied to a specific type of problem, situation, or region. This means that, while building an overall system for analyzing global change, the subsystems, theories, and assumptions used in those should be irrespective of aggregational, regional, or temporal differences. In other words, the subsystems, theories, and assumptions must be applicable at different levels of spatial aggregation and for different regions in different periods. Evidently, the claim on genericity cannot be fulfilled entirely, because a highly aggregated approach lacks specific regional/local dynamics. For example, for a commodity like oil with an increasingly global free trade context, the depletion and learning dynamics of exploitation of the global resource base can be dealt with adequately at the global level. However, to deal adequately with the import- and export flows of oil and their impacts on economic performance, require desaggregation to the level of economically, politically, and 
institutionally relevant actors and dynamics. This justifies the approach that, when desaggregating, region- and local-specific dynamics and data should be used and suited to the global dynamics.

\section{P-S-I-R Mechanism}

Adopting a systems-based approach as a guiding principle, a simple and transparent conceptual framework is proposed which consists of two strongly interconnected subsystems: the human subsystem and the environmental subsystem, each of which has, in turn, been split up into subsystems which are all interlinked. The whole framework is considered as a system of reservoirs and processes connecting the reservoirs, representing interrelated sets of cause-effect chains. Many of these processes are cyclic nonlinear or otherwise qualitative in nature. Both are influenced, directly and indirectly, by human intervention. Exogenous and endogenous developments perturbing the system, as well as interventions due to human activities, can lead to irreversible changes in the system. In order to create order in the whole suite of subsystems, the Pressure-State-Impact-Response mechanism (P-S-I-R) is used. The approach chosen here is very similar to the Pressure-State-Response framework developed by the OECD (1993). In fact, the "state" part of the OECD approach is split into two parts to be able to accommodate information about function changes (impacts) from changes in the state of a system. Although it is realized that the framework reprisents only one characterization of the global change issue, it is based upon a plausible division of the cause-effect chains of global change into subsystems:

Pressure System

represents the social, economic, and ecological driving forces underlying the pressure on the human and biospheric system.

State System

represents the physical, chemical, and biological changes in the state of the biosphere, as well as the changes in human population and resource/capitals.

Impact System

represents the social, economic, and ecological impacts as a result of human and/or natural disturbance.

\section{Response System}

represents human interventions as response to ecological and societal impacts.

As illustrated in Figure 5.2, the P-S-I-R mechanism forms a dynamic cycle. A.t various aggregation levels, the P-S-I-R mechanism can be applied to the global. 
system. Figure 5.3 shows the application at the highest aggregation level. At a lower aggregation level, the subsystems can recursively be broken down into more detailed subsystems, using the same P-S-I-R mechanism, if possible, etc.. This kind of conceptualization is useful for approaching an understanding of the dynamics of the complex global system. The human system includes the economy/energy and the human health subsystem, whereas the environmental system comprises the subsystems of water and element cycles, as well as land use changes.

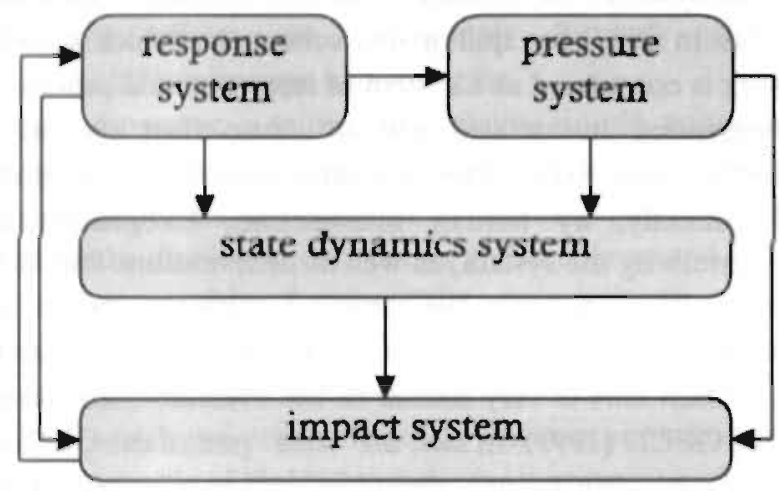

Figure 5.2: Systems diagram of the Pressure-State-Impact-Response chain.

Realizing that the P-S-I-R mechanism is only one way of ordering systems and their interlinkages, it is a plausible division of the cause-effect chains of global change into subsystems. The P-S-I-R mechanism shows that changes unleashed in one subsystem result in a cascade of events which may eventually find their way back to impact the starting point. For instance, while demographic and consumption pressures lead to changes in land use and energy consumption, such activities also lead to environmental and economic impacts, which may play significant roles in the future evolution of demographic and consumption patterns.

The P-S-I-R notion, however, is a relative one and dependent on the temporal, spatial, and aggregation scale chosen. In practice, this means that the P-S-I-R components are interchangeable and dynamically interwoven in such a way that there is no beginning and no end, constituting and inextricable continuum of interconnections. A major challenge is to make evident that impacts as result within the human system can form the pressure mechanism for the environmental system and vice-versa. 


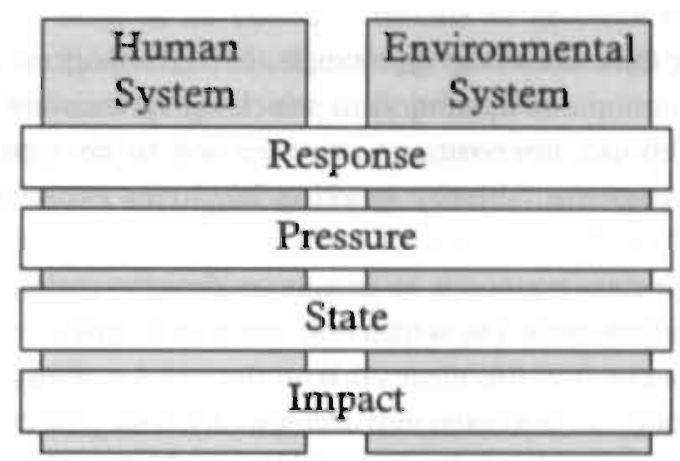

Figure 5.3: Conceptual framework for global change.

\section{Subsystems}

The human system is only a meager reflection of society's structure with its complex social, institutional, political, and cultural interrelations and dimensions. Current knowledge of the functioning of society and the role of humans in it is still in an immature phase, preventing an adequate conceptual representation, let alone an implementation that could be validated sufficiently. In the conceptualization, there is a focus hitherto on various facets of demography (social, economic, and epidemiological aspects of the size, structure and growth of human population) and its relations with the energy/economic sector (production, consumption, and long-term exploitation of our natural resources). This prototype facilitates future extension towards a more comprehensive consideration of human behavior, addressing issues as: what are the major determinants of human bebavior, can we develop and quantify appropriate social indicators, such as quality of life? (e.g. Jager et al. 1996a+b).

The environmental subsystem, sometimes confusingly and incorrectly denoted as the natural system, covers two subsystems. One subsystem, entitled composite pollution, delineates the variety of flows of natural and man-made substances, from basic elements to toxic pollutants, between the major reservoirs: the atmosphere, the ocean and the terrestrial biosphere (including soils). The ecosystem-related processes are included in the ecosystem health subsystena, which dynamically describes the fate of highly aggregated forms of both aquatic and terrestrial ecosystems. It outlines the quality of managed and unmanaged ecosystems, as a result of, in the first instance, its autonomous dynamics, and on top of this, human disturbances, such as transfers of land and water through agricultural-, livestock-, infrastructural-, and industrial- 
activities, and through socio-economic activities as urbanization, migration, and lifestyle changes.

One of the major difficulties is to disentangle the anthropogenic changes from the natural changes (autonomous dynamics) to which ecosystems are subjected. In the conceptualization, human interventions are supposed to be superpositioned on a "steady-state" of ecosystems, thereby ignoring long-term evolutionary changes of ecosystems.

The response subsystem represents an imaginary decision-making world, including a variety of human interventions by which both the human and environmental system can be influenced. In practice, this means that autonomous actions and activities, such as farming and hunting, are not taken into account. Without pretending to be realistic, as a first step, the institutionalized decision-making world is conceptualized by simply supposing one world governmental actor, which allocates capital goods. Following simple allocation decision rules, the total amount of capital goods is distributed towards different sectors: financial measures (taxes) for the energy/economy sector, and investments in infrastructure, technology, research and development, education, and family planning. The allocation mechanism controls that the annual stream of capital goods forms a closed system.

\subsubsection{Aggregation Level}

An important aspect of the TARGETS 1.0 model version is the top-down approach. TARGETS has been developed as a generic framework, so that the same basic framework might be applied at different spatial scale levels and with different degrees of spatial differentiation. To start with, the TARGETS generic framework has been operationalized for the world as a whole. The rationale for applying the TARGETS model at the global level is that major relationships and feedback mechanisms between the human and environmental system can be taken into account. Starting at a lower scale level would exclude a number of global mechanisms and processes. At a local scale, these global phenomena can at best be taken into account as exogenous scenarios. Besides, many "regional" mechanisms also have global components, due to long-distance transport mechanisms.

In the global version [version 1.0] of TARGETS, both the distributions on a scale below the temporal resolution in the model and the hetereogenities below the spatial resolution level of the model are dealt with by introducing classes and spatial distribution functions. The TARGETS model utilizes aggregated data and processes which are provided by theme-specific integrated models such as the IMAGE (climate assessment) and the RAINS (acidification assessment) models. Theme-specific 
integrated models, in turn, make use of data and processes generated by aspectcompartment models, such as air pollution models or groundwater models on the local or national scale. TARGETS is a highly aggregated and highly integrated model which is only possible when using the simplicity principle.

Representing global dynamics in a highly aggregated way has the advantage that an overall picture can be drawn of global change, including most relevant processes. Apparently, the high aggregation level has also serious drawbacks. In particular the local- and regional-specific dynamics, as well as the inter-regional processes, cannot be taken into account. Therefore, in the next step of the modelling strategy, the global version will be disaggregated and adjusted to regional circumstances, using regional data sets. In practice, this means that a few countries will be selected which may be representative for the intricate, dynamic interplay between the social, economic and environmental changes in the longer term. Presumably, a few countries are chosen that characterize the problematic nature in the developing countries, such as India and China, and some countries which portray the transition pathways of the developed countries, such as the USA and the Netherlands. In practice, this means that such case studies pursue the question of what sustainable development on the regional level means. It is by no means intended to fully disaggregate the TARGETS model for all world regions and countries.

With respect to temporal aggregation and disaggregation, the overall time horizon for the TARGETS 1.0 model spans two centuries. The simulation starts at the beginning of this century, the year 1900 , symbolizing the end of the pre-industrial era and ends at the end of the next century, the year 2100 , involving three different future generations from today.

The general time step within the TARGETS 1.0 framework is one year, albeit this varies per submodel. Some physically related processes require a time step of one month, or of a season, whereas others can be aggregated on a yearly basis. TARGETS is implemented and developed in the modelling environment and simulation language M (De Bruin et al., 1996), which is ultimately converted into C. It runs as well on a workstation (UNIX) as a PC (MS-Windows), and a user-friendly interface guides the user through the concepts and the model structure.

\subsubsection{Calibration and Validation}

The validation process of a model like TARGETS is a trade-off between accuracy and utility. Validation within the TARGETS context means that each submodel should be scientifically valid, in the sense that the model structure, relations, 
parameters, and dynamic behavior over the period 1900-1990 (or if necessary a briefer period) reflect the prevailing theoretical insights and the key facts pertaining to that part of reality which the model is supposed to represent. It is of major importance that the various submodels are calibrated and validated at the regional or country level. Therefore, experts in the field of small-scale, detailed models in a specific region or country are asked to analyze the model results and to validate regionalized model results for a specific world region against regional datasubsets. In view of the generic structure of the submodels, the experts should indicate the extent to which the generic structure of the module(s) should be adapted to regional conditions and dynamics.

A second way of validating the submodels is by means of the intercomparison with expert model which they are supposed to represent at the meta-level. An example of validating a simple carbon cycle model (based on Goudriaan and Ketner, 1984) against observational data and more complex two- and three-dimensional carbon cycle models is presented in Rotmans and Den Elzen (1993). Having validated the submodel, the overall validation of the modelling framework should be performed, whereby special attention should be devoted to the validation of the coherence and internal consistency of the model.

\subsection{Horizontal Integrative Description of TARGETS}

Horizontally, the TARGETS model can be subdivided into combinations of various pressure submodels; combined state dynamics submodels; combined impacts submodels; and various response submodels. Below, a generic description is given of the potential interlinkages between the pressure model; between the state dynamics models; between the impacts models; and between the response models.

\subsubsection{Pressure Models}

Global pressure models are intended to chart the driving forces behind the increasing worldwide pressure on the environment. They shed some light on the interdependencies, in terms of their nature and scale, which exist among factors such as growth in population, developments in the stock of capital goods, use of resources, growth in production, and technological development.

In TARGETS, all causative or pressure models describe the developments in human population, energy/economy, land use, and water use, respectively, where all developments are interlinked. This is demonstrated in the descriptions of the separate modules in Section 5.4, which show common pressure systems, in which the pressure 
system within a specific module is always composed of pressure elements arising from a number of other modules.

\subsubsection{State Dynamics Models}

The state dynamics models describe the biochemical status of the environmental system and the social and economic status of the human system, which are, of course, strongly interrelated. The biogeochemical status of the Earth is characterized by an interconnected complex of transport mechanisms and transformation processes, many of which are of a cyclic nature. Biogeochemical cycles describe the transformation and movement of chemical substances in the global environment. An integrated model is being developed that delineates the physical, chemical, and biological state of the biosphere. An essential part of the model is the integration of the element cycles $(\mathrm{C}, \mathrm{N}, \mathrm{S}$ and $\mathrm{P})$ and the interactions between the cycles in the biosphere. Other cycles implemented are the global hydrological cycles and global cycles of some representative heavy metals, pesticides, and organic micropollutants. All these biogeochemical cycles are related to important global and regional issues: climate change, soil degradation, acidification, stratospheric ozone depletion, and dispersion of toxic and persistent micropollutants. This will be done by coupling the various compartments: the atmosphere, terrestrial biosphere, lithosphere (soils), and the hydrosphere (oceans, coastal seas and rivers); and subsequently integrating the physical, chemical, and biological interactions between the diverse compartments.

This global disturbance of the biospheric system interferes with the changing state of the population and resources reservoirs of the human system. The size of the demographic reservoirs subsystem is determined by the fertility rates, and disease and age-specific mortality levels. The mineral and fossil resources reservoirs are determined by human decisions and behavioral rules, such as resource exploitation through investments by private companies or by government planning.

\subsubsection{Impact Models}

The impact models can be divided into two types of strongly interrelated models. Disturbance of the physical, chemical, and biological cycling of basic elements and other chemicals may lead to climate change, acidification, stratospheric ozone depletion, eutrophication, and air pollution. Together with land use change, biomass burning, erosion, fertilizer use and water flow change, it may affect the ecological health and food and feed supply. 
The estimation of effects of determinants such as socio-economic developments and changing global environmental conditions on human health is important. Global environmental change may directly effect the presence of micro-organisms causing diarrhea and malaria; entomological, bacteriological, and viral contamination and the presence of vector-borne diseases; suppression of the body's immune system that might lead to increases in non-melanoma and melanoma skin cancer incidences and cataract. Indirectly, global environmental change affects human health via the world food supply, which may lead to premature death, infectious diseases or certain degenerative diseases. Socio-economic development affects prevention (immunization, water supply and sanitation and vector control) and treatment (therapies) of diseases, and, therefore, meaningfully changes the palette of determinants.

\subsubsection{Response Models}

The response model contains those variables by which the user can influence human activities or/and the environment. The response variables are clustered into financial and legislative measures. An essential part of the response model is the allocation model, which distributes the total amount of capital towards the different sectors and controls that the annual stream of capital goods forms a closed system. While the socio-economic component of TARGETS is underdeveloped, the response model is highly premature.

\subsection{Vertical Integrative Description of TARGETS}

Vertically, the TARGETS modelling structure can be subdivided into various autonomously functioning submodels, called modules. Each module represents the cause-effect relationship for a particular theme of global change. The modules describing the human subsystem are the population and health module and the energy/economics module, whereas the environmental subsystem is described by the global cycles, land/soil, and water modules.

\subsubsection{The Global Population and Health Model}

The aim of the population and health model is to simulate the changes in morbidity and mortality levels under varying social, economic, and environmental conditions (Niessen et al., 1996). The overall health transition, consisting of the demographic, epidemiological, and fertility transition, is simulated. The generic structure and build- 
up of the model enables implementation at various levels, from the global to the country level. Based on a number of socio-economic and environmental determinants, the fertility and population levels as well as ten different diseases, among which are respiratory, cardiovascular, cancer, and vector-borne diseases, are calculated. The horizontal element comes into play by considering a limited number of relevant combinations of risk exposures and combinations of diseases. Following the P-S-I-R mechanism, Figure 5.4 gives the structure of the population and health model:

(i) A pressure submodel represents those factors determining the fertility level, the health risks and causes of death. These factors consist of socio-economic and environmental factors. The socio-economic pressures are GNP and female literacy level, while the environmental pressures are food and water availability, global climate change and changes in UVB-radiation;

(ii) A state submodel which consists of three submodels: the fertility module, the population module, and the disease module. The main outcome of the fertility model is births by sex as a function of marriage, contraceptives, abortion and postpartum infecundability, all of which are driven by the human development index; the population module calculates the number of births and deaths for each of the five age groups; the disease module calculates the diseased state of the population;

(iii) An impact submodel which represents the quantitative and qualitative aspects of the population. The quantitative aspect mainly consists of the size of the total population. The quality of the population is measured by the life expectancy and the disability adjusted life years (DALY);

(iv) A response submodel which includes responses concerning fertility behavior, population policy, and investments in health care. Response options for fertility behavior are: mass communication, education, family planning, and abortion policy. The level of bealth services is endogenously determined by the value of total DALIES lost modified by micro and macro conditions. The health services are allocated via an allocation function to primary and secondary prevention ancl curation. 


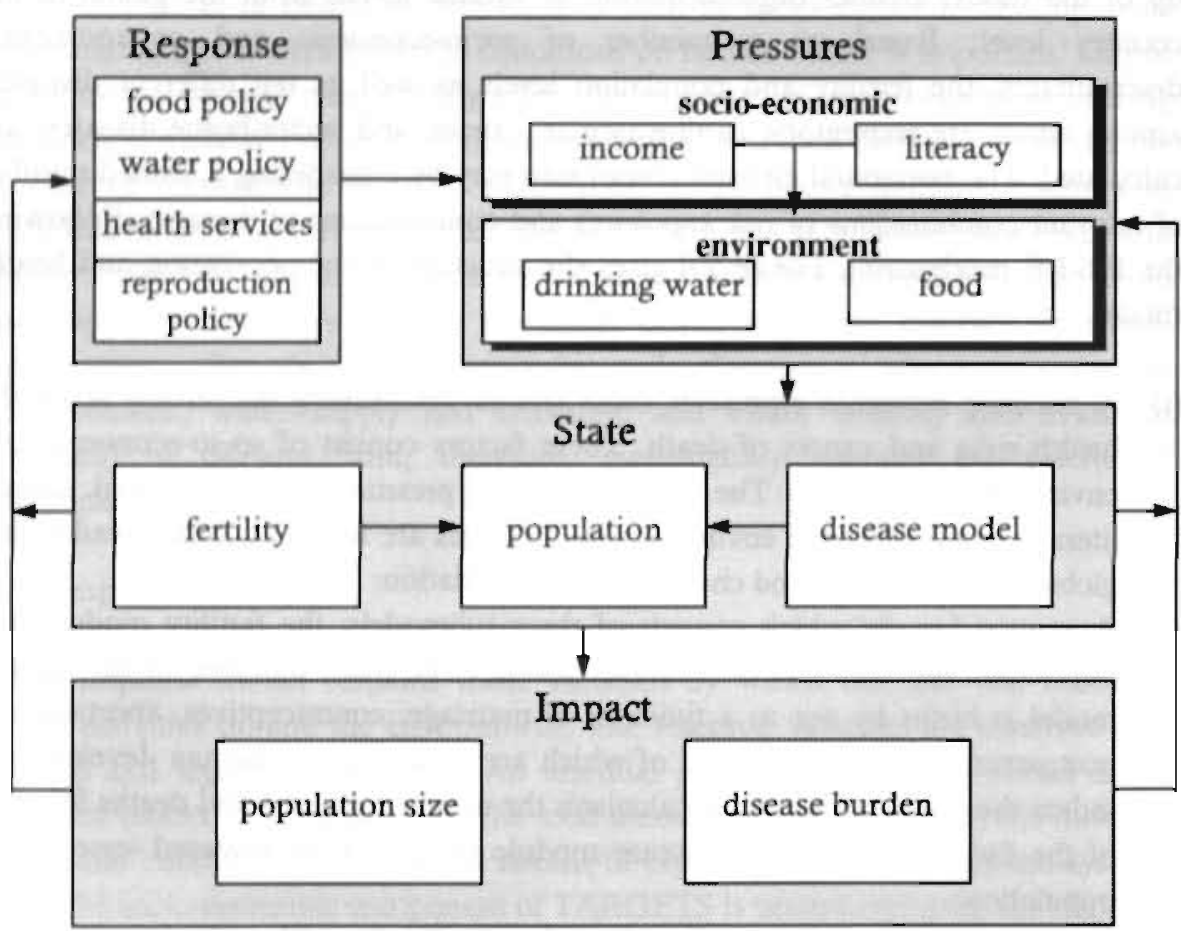

Figure 5.4: The integrated population and health module.

\subsubsection{Energy/economics Model}

The Energy model has been developed for three principal reasons: i) to analyze the long-term dynamics of energy conservation and the transition to non-fossil fuels; ii) to simulate the energy system within an integrated long-term modelling framework; iii) to formulate long-term sustainable energy policies from an integrated perspective (de Vries and van den Wijngaart, 1995). The Energy model consists of five submodels: Energy Demand, Electric Power Generation, and the Supply of Solid (SF), Liquid. (LF) and Gaseous Fuels (GF). The very simple economy submodel consists of an industry, services, and consumers part. Industrial output is generated at a fixed capital-output ratio; labor is not taken into account; industrial output is allocated to the other modules of TARGETS 1.0.

An overview of the Energy/economics model according to the P-S-I-R scheme is given in Figure 5.5: 
(i) A pressure submodel calculates the demand of a number of commercial fuels (solid, liquid and gaseous) for five separate economic sectors: residential, commercial/services, industrial, transport and other. The demand for electricity is calculated for the economy as a whole. For each of these sectors, first the expected activities are given exogenously by scenarios. To calculate sectoral energy demand, these activities are multiplied by the sectoral energy intensity influenced by: i) structural change; ii) autonomous energy efficiency improvements; and iii) price-induced efficiency improvement. The last two factors represent technological development. The sectoral energy demand is translated in a demand for the different kinds of fuels of the Energy model (gaseous, liquid, and solid fuels) based on a price-based allocation: a type of fuel that becomes relatively expensive will be used less.

(ii) A state submodel consists of the Primary Energy Production Modules (the LF/GF/SF Models) and the Electric Power Generation Module. Substitution between various fuels is price-driven. Important features in the Energy Production modules are the learning rate and depletion multiplier, which influence the production costs. To a lesser extent, the assumed reserves are important since they finally limit the amount of fuel that can be used. For the altemative fuel (biofuel), the production costs are determined by learning by doing, depletion (of available land), and capital-labor substitution.

The Electric Power Generation Model represents the generation of electricity by way of thermal, non-thermal and hydropower generating capital stocks. The thermal generation of electricity uses solid, liquid or gaseous fuel depending on their relative prices. Non-thermal electricity generation is viewed as a competing alternative source, representing nuclear, solar energy or others (not hydropower) which penetrate the market. The costs of non-thermal electricity production depend on learning by doing.

(iii) An impact submodel generates yearly emissions of a number of gases, most of which are related to global climate change. For the following compounds, the emissions patterns are simulated: carbon dioxide $\left(\mathrm{CO}_{2}\right)$; carbon monoxide (CO); methane $\left(\mathrm{CH}_{4}\right)$; nitrous oxide $\left(\mathrm{N}_{2} \mathrm{O}\right)$; nitric oxide and nitrogen dioxide $\left(\mathrm{NO}_{\mathrm{x}}\right)$; and sulphur dioxide $\left(\mathrm{SO}_{2}\right)$. Furthermore, the impact madel generates requirements for the amount of land used for the alternative fuels (biofuel), which is a basic input for the TERRA model of TARGETS.

(iv) A response submodel which includes responses concerning allocation of industrial output and the allocation of fuels to meet the energy demand. For 
institutional reasons, TARGETS do not contain a macro-economic model, but just a simple transparent allocation model for sectoral investments based on World 3 (Meadows et al., 1974). The industrial output is allocated among consumption and investments in industry (reinvestment), services (including health-services), agriculture, and energy. Within AQUA, investments are related with energy (hydro-power), agriculture (irrigation), and health-services.

Within the energy modules, R\&D programs are used to stimulate alternative fuels and carbon tax policy to reduce energy demand and stimulate non- $\mathrm{CO}_{2}$-emitting fuels.

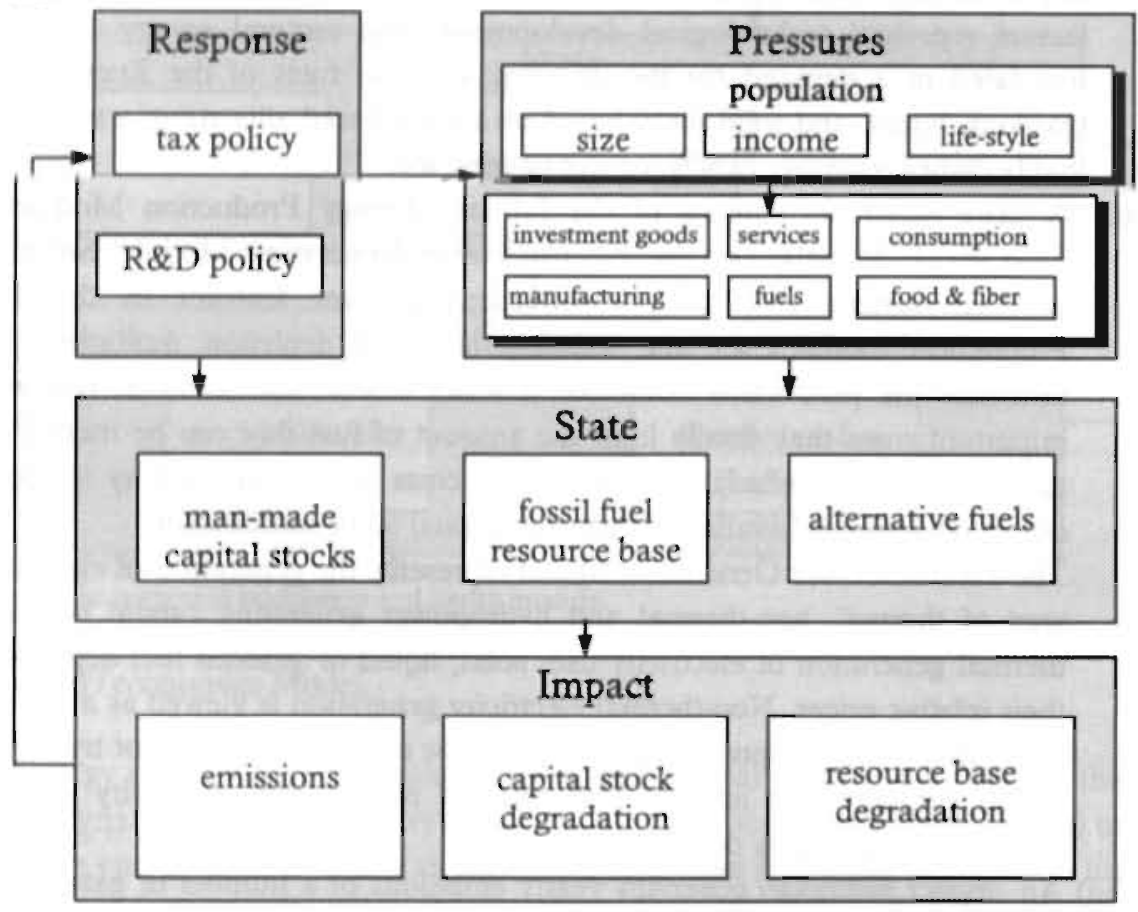

Figure 5.5: The energy/economics module.

\subsubsection{The Global CYCLES Model}

The CYCLES model describes highly aggregated the whole cause-effect chain of the global biogeochemical cycles, by coupling the causes (social, demographic, and economic pressure), dynamics (physical, chemical, and biological changes in state) of the flows of elements between and within the various compartments, and the impact 
of the perturbed cycles on the global environment. The model allows for quantification of those interlinkages and feedbacks between the global cycles, which are reasonably well known. Of the six basic elements determining life on earth, (C, N, $\mathrm{P}$, and $\mathrm{S}$ ) have been simulated, $\mathrm{H}_{2} \mathrm{O}$ (the hydrological cycle) is described in the AQUA model, while $\mathrm{O}_{2}$ has not yet been taken into account. Next to the basic elements, the global cycles of some representative toxic substances (DDT, PCBs, lead, phthalates) and other chemicals (CFCs and alternatives, carbon tetrachloride, methylchloroform, and tropospheric ozone and its precursors) are also included.

Following the P-S-I-R systems approach, the CYCLES model is subdivides as follows (see Figure 5.6):

(i) A pressure submodel which describes the driving forces underlying the human perturbations of the element cycles and other chemicals: emissions and flows of compounds of C, N, P, and S from the energy and industrial sector, land use changes, biomass burning, erosion, fertilizer use, harvesting, and water flow changes;

(ii) A state submodel which describes the physical, chemical, and biological cycling of the basic elements and other chemicals within and between the atmosphere, terrestrial biosphere, lithosphere (soils), and hydrosphere (fresh surface waters, including lakes and rivers, and oceans);

(iii) An impact submodel which describes the impacts of the perturbed cycles and chemical substances on the global environment. The model comprises the following submodels:

- a climate assessment module, which simulates the radiative forcing and globalmean temperature changes due to changes in concentrations of greenhouse gases and sulphate aerosols;

- an ozone assessment module, which uses as input atmospheric chlorine and bromine concentrations and calculates stratospheric ozone depletion, increased UV-B radiation, and skin cancer incidences;

- A soil acidification assessment module, which, using depositions of soil-acidifying compounds as input, calculates the soil acidity, taking into account acid load and the load's buffering capacities.

(iv) A response submodel is not contained in the CYCLES module. The relevant responses options are included in the other modules of TARGETS.

In spite of the global, highly aggregated spatial resolution level the CYCLES model operates on, a number of spatial disaggregations are used. The atmosphere is 
subdivided into troposphere and stratosphere; the oceans into two mixed-surface layers and 5 deeper layers; while the terrestrial biosphere has been disaggregated into specific classes for soil (soil productivity), climate (length of growing period), and land use (six ecosystem types or land cover types).

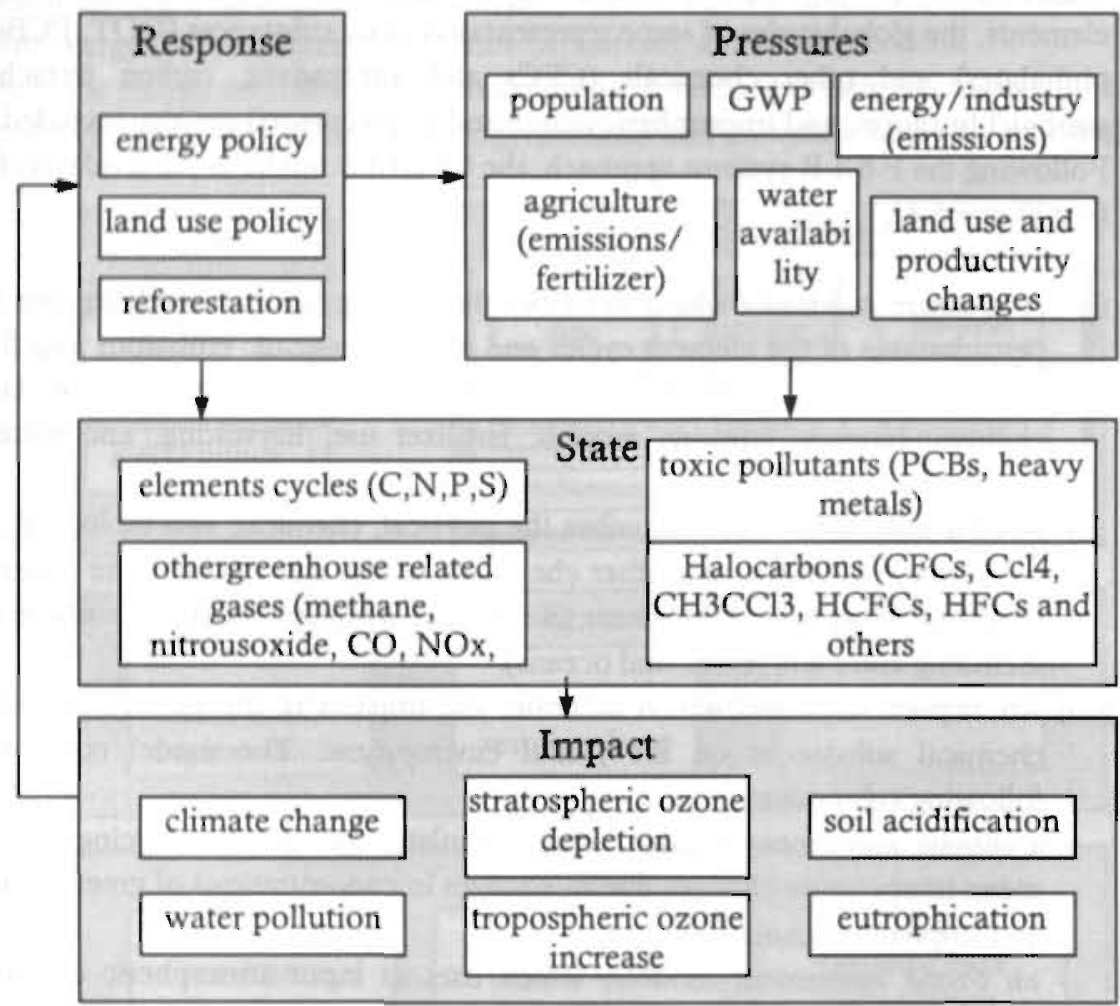

Figure 5.6: The CYCLES module.

\subsubsection{The Global TERRA Model}

The integrated land model TERRA attempts to shed some light on the complex dynamics of the interplay between population growth, economic growth, land management, and food supply, on one hand, and global environmental change, on the other hand. The model may provide a better understanding of the human pressure on the global land and food system, and the impacts of changing food supply conditions on human health and economics. Figure 5.7 shows the model as structured according to the P-S-I-R schematization: 
(i) A pressure submodel describes the driving forces underlying the continuously changing pressure on the land and food system. It is characterized by the food and non-food demand consisting of the demand for vegetable and animal products, the tropical wood demand (fuel wood excluded) and the demand for urban area. All demands are a function of economic growth as indicated by the Gross National Product (GNP) and the population size. Other (environmental) pressures considered are: water availability for irrigation, climate change, and land degradation;

(ii) A state submodel describing the changes in the physical state of the land in terms of land use and quality changes on food production as a function of the environmental and socio-economic pressures, and different land policies. Three main parts or modules can be distinguished in the state system: (i) land use/land cover dynamics; (ii) erosion and climate change; and (iii) food and feed supply;

(iii) An impact submodel describing the impacts in terms of food supply per capita where the actual impact is on the level of DALY lost as calculated by the population and health model; the loss of natural ecosystems (i.e. forests and natural grasslands), and land degradation ultimately resulting in the loss of arable land;

(iv) A response submodel giving the user the option to specify different (agricultural) policies which are: land clearing, expanding the area of irrigated arable land, intensification on irrigated land (i.e. usage of fertilizers and other inputs), land or soil conservation and reforestation. The total agricultural investments are the result of these policies, plus the intensification on rainfed arable land, which is not a result of a policy, but calculated by the state system.

In the TERRA model, a number of spatial heterogenities are introduced by disaggregation into specific classes for soil, climate, and land use. Eight land cover types have been distinguished: natural forest, grassland (pasture land included), rainfed arable land, irrigated arable land, urban area, degraded area, reforested area, and other lands (wetlands, deserts and ice caps). Next, the land cover types have been disaggregated into 18 parts, based on the following classes:

(i) Two economic or temperature zone classes: the developing and industrialized countries or tropical and temperate zone;

(ii) Three length growing period (LPG) classes: short (less than 90 growing days); medium ( 90 to 240 days); and long (more than 240 days);

(iii) Three inherent soil productivity (Q) classes: low, medium, and high. 


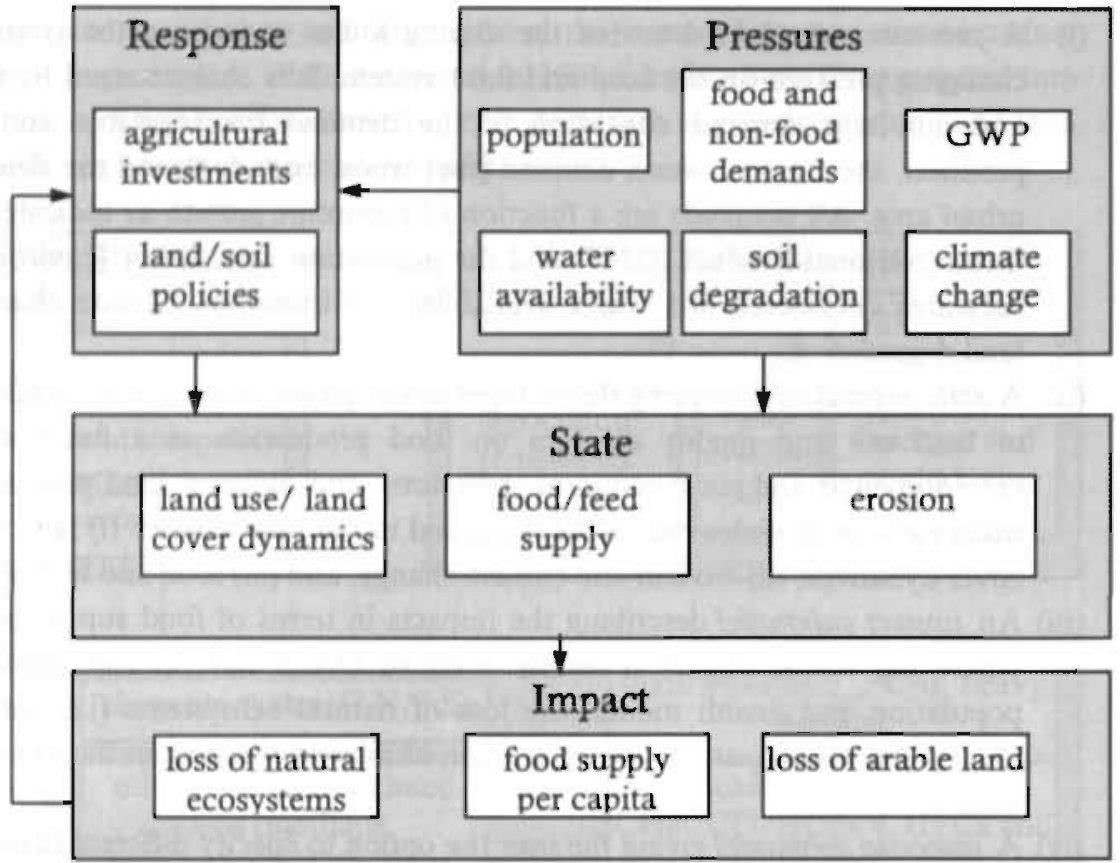

Figure 5.7: The TERRA module.

\subsubsection{The Global Water Model AQUA}

AQUA takes account of the functions of the water system that are considered the most relevant ones in the context of global change. Human-related functions considered are domestic, agricultural and industrial water supply, hydroelectric power generation, and protection against flooding. Ecological functions taken into account are natural water supply to terrestrial ecosystems and protection of aquatic ecosystems. Water system. functions that are not taken into account in the present version are fishery, aquaculture, transportation, and recreation.

The main model structure of the AQUA model is presented in Figure 5.8. The four main submodels distinguished are:

(i) A pressure submodel describing both socio-economic and environmental pressures on the water system. In the pressure submodel, total water demand is calculated as a function of, among other things, population size, gross national 
products, industrial production, demand for irrigated cropland, and water supply efficiencies. Further, it is simulated which fractions of the water used return to ground or surface water and which fraction gets lost through evaporation. Part of the waste water is treated before discharge to the surface water.

(ii) A water system submodel simulating hydrology and fresh water quality. In the water system submodel, the hydrological cycle is modelled by distinguishing ten water reservoirs (fresh surface and ground water, atmospheric water, oceans, etc.) and water flows between these reservoirs. Among the outcomes of the model are time series for net precipitation, river runoff, ground water level decline, fossil ground water depletion, and sea level rise. The water quality is described in terms of nutrients, heavy metals, pesticides, and organic micropollutants. Four water quality classes are distinguished to characterize the fresh water storage in functional terms.

(iii) An impact submodel describing impacts of water system changes on water supply, flooding risks, and the functioning of ecosystems; The impact submodel described the performance of the various functions of the water system. The actual water supplies to households, agriculture, and industry are calculated, as are the generation of hydroelectric power and the impacts of sea level rise on world's coasts. Besides, the impact submodel simulates the water supply to terrestrial ecosystems and the protection of aquatic ecosystems.

(iv) A response submodel representing the human response to negative impacts and providing the possibility to add extra water policy measures. The response model simulates expenditures demanded and actually made (e.g. in infrastructure, technology, and education), financial measures (e.g. water pricing) and legislative and managerial measures, partly as autonomous developments and partly as policy influenced developments. 


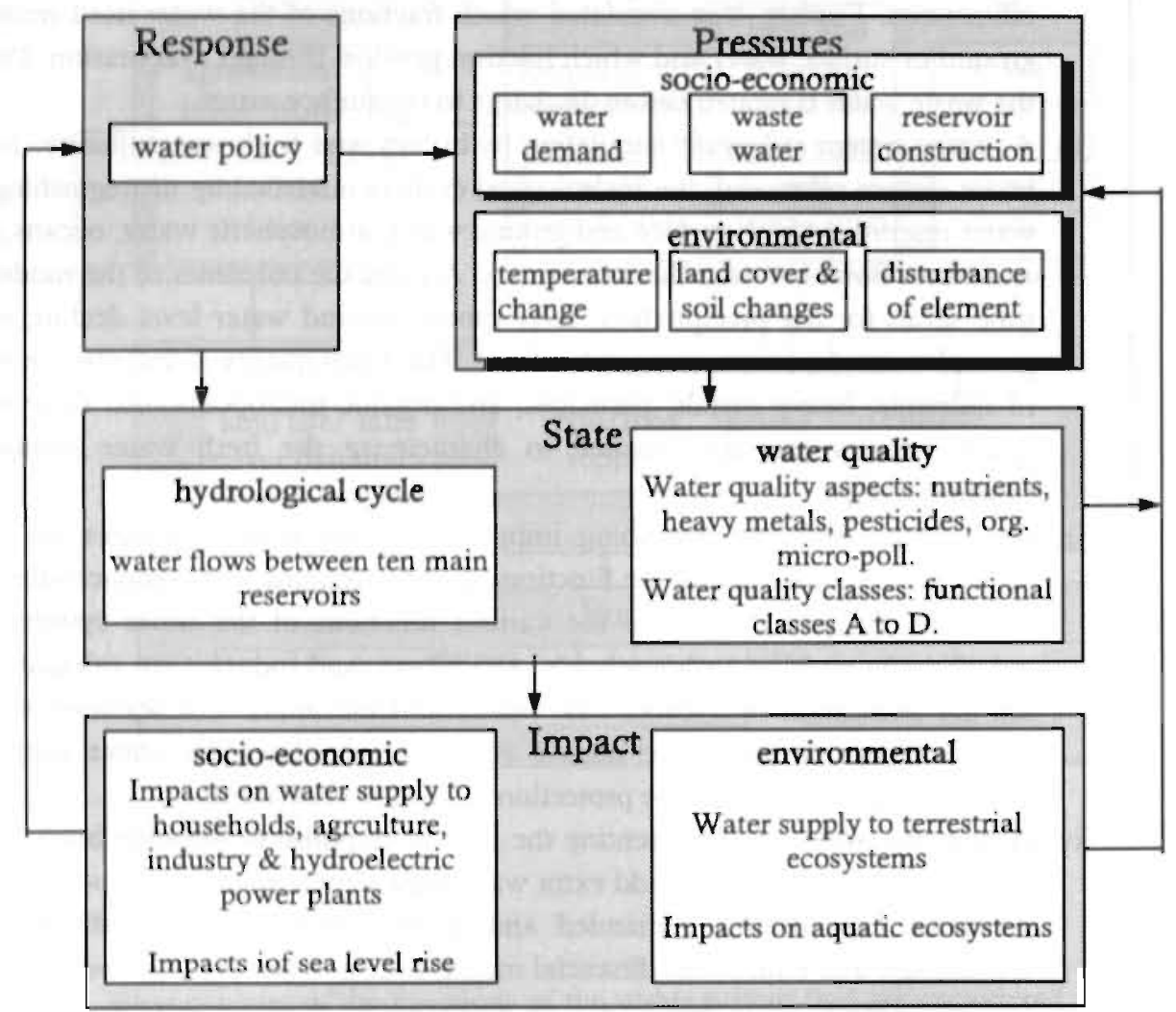

Figure 5.8: The AQUA module.

\subsection{Interlinkages Between Submodels of TARGETS}

During the simulation period, data flows between the different submodels each time step. This is essentially different from a lot of other computational frameworks in which complete time series from one are used in a subsequent model. Applying TARGETS, therefore, implies that interactions and feedback loops between different phenomena are taken into account. In Figure 5.9, an overview of the flow exchanges, in terms of input and output, between the different submodels, is depicted. 


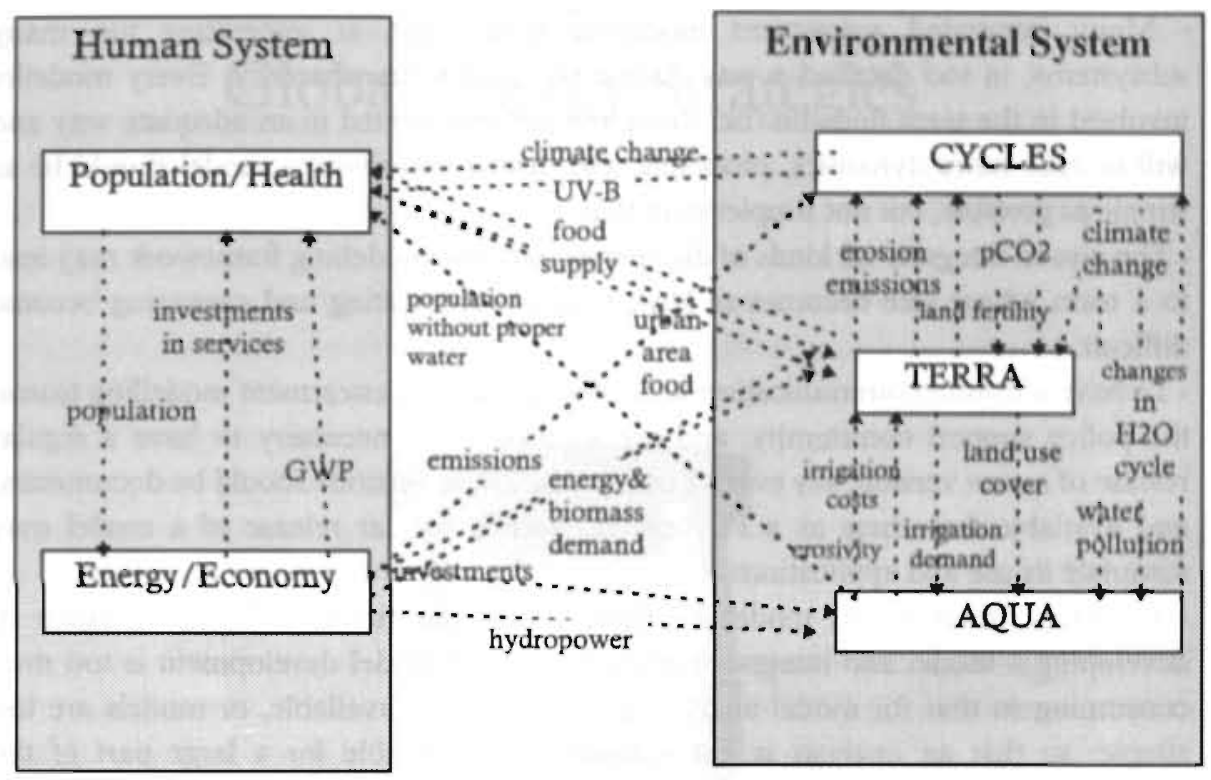

Figure 5.9: Overview of main interlinkages within TARGETS.

\subsection{Retrospective}

During the two years I have served as a model integrator of 'TARGETS (spring 1993 - spring 1995), trying to integrate the various modules into one interactive model framework, I have experienced what an incredibly difficult task it is, as others did in the period thereafter. In this Section, I go back to the integrated modelling process and make some remarks based on the experience of working within the TARGETS modelling team and the contacts with other integrated modellers outside the Netherlands. These remarks are meant to stimulate and improve the challenging, but complex process of integrated assessment modelling.

As was discussed in introductory Chapters, integrated assessment modelling is a rather young discipline, whose methodology is in its embryonic phase. I notice that:

- The important problem in interdisciplinary research is the fact that every modeller, trained in his/her own discipline, speaks his/her own "language" causing barriers between the disciplines which cannot be broken easily. Therefore, an integrated. assessment modeller needs, in addition to being skilled in his/her original discipline and interest in others, to be patient and punctual in communication. 
- Many integrated assessment modelling teams aim at integrating too many subsystems, in too detailed a way, losing the aspired transparency. Every modeller involved in the team finds his/her discipline not represented in an adequate way and will include more dynamics, processes, and details. However, a model should be as simple as possible, but not simpler than that.

- The aim to integrate all kinds of disciplines into one modelling framework may lead to a team whose size becomes so large that communicating and managing become difficult.

- To have a fruitful communication with other integrated assessment modelling teams, the policy support community, and the sponsors, it is necessary to have a regular release of a new version, say every 1 or 2 years. These versions should be documented and available for others as a PC-version. Such a regular release of a model may stimulate its use and applications.

- Currently, there is no modelling team which has found a fruitful balance in developing a model and integrated assessment. The model development is too time consuming so that for model analysis too little time is available, or models are too simple, so that an analysis is not reliable and acceptable for a large part of the disciplines involved.

- Currently, there is no operational model which balances economics, social, and ecological dynamics in an appropriate way. TARGETS, for example, has an adequate description of parts of the environmental system, the population and health system, and the energy system, but does not yet adequately represent the social and economic dynamics. Various models rooted in an economic background (e.g. DICE, MERGE, CETA, PAGE) do represent the environmental system in a less adequate way.

- Scientific reliability competes with political and organizational credibility. Institutional interests explain why some models focus on environmental and health issues, while others focus on economic aspects.

- To be useful for application at the international level, a regionalized version is desired. However, this should be carefully balanced with the many uncertainties, avoiding the misleading image of a truth-machine.

- Current integrated assessment models mainly deal with macro-dynamics on a global scale. While the many dynamics on the local scale influence the global dynamics and visa-versa, and because of the different type of dynamics, there is a need for the development of integrated assessment models with local dynamics on a local scale.

- The development of an integrated model is too much the goal of research projects, although it should be serving only as a tool to support integrated assessment. 


\section{6 \\ Global Energy Strategies ${ }^{1}$}

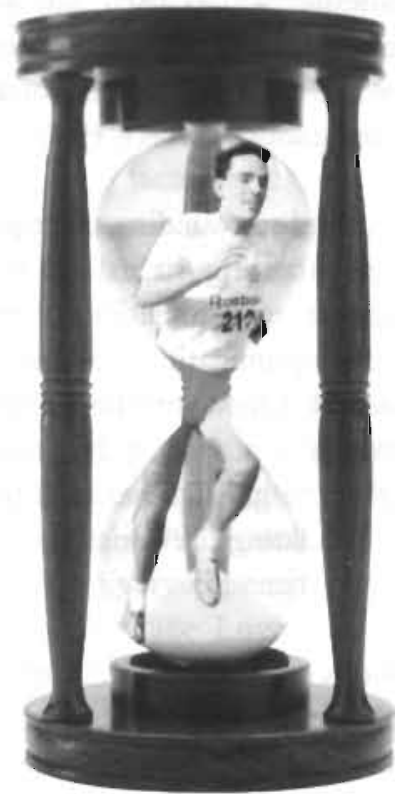

1 This Chapter is based on De Vries and Janssen (1996) and Janssen and De Vries (1996). 


\subsection{Introduction}

The Framework Convention on Climate Change of the United Nations (1992) has as its stated goal to achieve stabilization of greenhouse gas concentrations in the atmosphere at a level that would prevent dangerous anthropogenic interference of the climate system. Concrete goals are not defined, but a widely used exercise is to examine the implications of establishing various ceilings for atmospheric $\mathrm{CO}_{2}$ concentration (e.g. Edmonds and Wise, 1995; Wigley et al., 1996). A stabilization target of 550 ppmv, a doubling of the pre-industrial level, is a widely used benchmark among climate researchers. Another widely used, but weakly underpinned, climate target is an absolute temperature limit of $2^{\circ} \mathrm{C}$ above pre-industrial level. This temperature limit can be viewed as an upper limit beyond which risks of considerable damage are expected to increase rapidly (AGGG, 1990).

Despite the many uncertainties surrounding the topic of climate change, there is a need to derive more insight in balancing the risks of a. possible climate change and the efforts of reducing greenhouse emissions. From this perspective, this Chapter describes a number of illustrative optimization-oriented experiments performed with the TARGETS model (Chapter 5). I focus on the energy-cycles part of TARGETS, that is, the energy module which is a system dynamics framework of simulating demand and supply of energy at the global level, and the cycles module which is a global, comprehensive model simulating the consequences of disturbing the element cycles.

Different sets of assumptions on technological developments are used to explore (global) energy policies which would meet climate change targets. The impact of uncertainties in economic and technological development on possibilities to meet the targets is investigated. Furthermore, the consequences of differences in the timingpattern of mitigating policies are investigated to illustrate the risks of doing too little or doing too much. This leads to the construction of several hedging strategies which explicitly take account of the possibility of different technology developments in the long run and balance short term actions, given those long term uncertainties. Finally, the discussion is broadened by including the acidification problem which is related to climate change by a common cause, $\mathrm{SO}_{2}$ emissions. But first, I briefly consider some methodological questions. 


\subsection{Bottom-up versus Top-down Modelling}

Top-down and bottom-up modelling techniques have been used to answer the question of how much it would cost to reduce greenhouse gas emissions. Bottom-up modelling requires detailed specification of energy-related and other technologies. In this approach, the present and future probable mix of technologies in each economic sector is described in terms of their costs, inputs and outputs, including emissions. The aggregation level may range from broad economic sectors down to individual plants. The advantage of this approach is that it allows specification of particular technical innovations. On the other hand, it requires huge numbers of technological coefficients and other data, which cannot easily be checked for consistency.

The macro-economic top-down approach with embedded input-output coefficients projects future emissions on the basis of neo-classical growth models with production functions, goods and factor markets and utility functions. Usually, optimization of discounted consumption is used to balance the costs and benefits of future activities. The aggregation level may range from simple models of the aggregate economy, through aggregate economic models with a more detailed energy sector, to full dynamic general equilibrium models.

The two types of models were conceived and designed from different disciplines, for different purposes and they lead to quit different conclusions (Wilson and Swisher, 1993). Top-down analysts like Manne and Richels (1992), Nordhaus (1994), Peck and Teisberg (1992), and Burniaux et al. (1991) conclude that even moderate steps toward mitigating global warming will be very expensive for society. They support a waitand-see policy. Bottom-up analysts like Lovins and Lovins (1991) and Williams (1990) conclude that much can be done to mitigate global warming at little or no cost to society and support a take-action-now policy stance. Wilson and Swicher (1993) conclude that the two ways of seeing and describing the world are conceptually incompatible, and the choices between wait-and-see and take-action-now will, therefore, be made on political, rather than scientific grounds.

As the macro-economic effects of energy policy are not included in the energy model of TARGETS, it has more components of a bottom-up than a top-down. approach. It is, therefore, expected that results will suggest that action-now will be. cost-effective. There are various arguments as to why early action is not necessarily costly or why future cost may not be lower (Grubb et al., 1995; Grubb, 1996). First, there are a wide range of options and technologies for limiting emissions, at varying cost levels and with different prospects for cost reductions. Even when "no-regrets" options, which can be implemented at no net costs, are exhausted, there are a wide range of options, including many cheap ones (Lovins and Lovins, 1991; Grubb, 
1996). Secondly, in macro-economic models, technology development occurs usually independent of market conditions. However, this is not a widely accepted hypothesis among economists who work on technology issues. Without early investments, future technology might not be available at lower costs. For example, Arrow (1962) noted that much knowledge is acquired through learning-by-doing and technology development is often induced by market circumstances. Thirdly, technological development tends to be strongly biased towards existing modes, which is called the lock-in effect (Arthur, 1994; Nakicenovic and Grubler, 1991). This causes industries with a large market share to spend large R\&D funds along established technological lines to protect their existing positions, thus creating barriers to more advanced and cheaper options. In view of such learning-by-doing dynamics, emission constraints may actually accelerate the development of cost-effective altematives. Fourthly, the inertia of capital stocks in energy-producing sectors make rapid changes costly. Power generation facilities, petroleum refineries, etc., have lifetimes of 30-40 years, suggesting that transition to alternatives have to start early. Infrastructural projects may have implications which are even more far-reaching.

\subsection{Methodology}

The problem addressed in this Chapter is to find out which strategies for $\mathrm{CO}_{2}$ reduction can meet certain targets with the least costs for the economy. As an indicator of costs, the performance of the energy system is measured in energy expenditures, which is defined as the sum of prices for fuels times the production of each fuel, plus the cost of efficiency improvements. Moreover, the energy expenditures are used as a percentage of GWP to compare the levels of expenditures from year to year. Thus, the objective of the adopted optimization problem is to minimize the energy costs relative to gross world output, aggregated over the period 1995 until 2100. Given this objective, additional constraints on environmental quality can be investigated, like $\mathrm{CO}_{2}$ concentration and global mean temperature increase constraints.

The decision variables which will be optimized consist of a carbon tax policy and R\&D programs in non-thermal electric and biomass fuels. The carbon tax increases prices of fossil fuels and will stimulate energy conservation and penetration of alternative fuels, while the R\&D programs stimulate the learning process, so that the production costs decline and the alternative options become more competitive in the energy market. 


\section{Problem Formulation:}

Minimize energy expenditures as percentage of GWP aggregated for the period 1995-2100.

Decision variables: - carbon tax (S/RC),

- non-thermal electric demonstration program (MWe/yr),

- bioliquid and biogas demonstration programs (EJ/yr).

Constraints:

- $\mathrm{CO}_{2}$ Concentration or Temperature Increase.

Although rule-based investment decisions characterize the system dynamics approach of the model as used, an optimization algorithm is employed to find a carbon tax policy and R\&D programs which steer the system to the preferred performance. A genetic algorithm is used to search for (sub) optimal solutions to the previously defined problem. By simulating a competition between scenarios to derive cost-effective ways to meet the policy targets, the algorithm generates new scenarios, leading to a family of scenarios of which the performance increases over time (Goldberg, 1989). Such an algorithm does not lead to better results in sound mathematical search spaces, but outperforms most of its cousins in noisy search spaces (Goldberg, 1989). While the TARGETS model shows complex nonlinear behavior, such a robust algorithm is desired. Another important aspect is the fact that the genetic algorithm treats the model as a black box, so that it can be used in the original appearance, which is attractive in evolving model versions in integrated assessment modelling. Finally, it is not the primary purpose to find the optimal solution, but to find, with help of an advanced simulation model, a suitable strategy which is as good as can be found in a limited amount of time. The optimization algorithm is, therefore, not more than a tool to search for specific scenarios.

\subsection{Four Global Energy Scenarios}

In recent years, a number of global long-term energy scenarios have been published, primarily to support the development of policies with respect to climate change. In general, these scenarios can be characterized as either "business-as-usual" (or "no-intervention", "reference" or "Conventional Wisdom") scenarios or "policy" scenarios. The scenarios usually capture a wide range of outcomes with respect to the main reported variables: energy supply and associated greenhouse gas emissions. The differences between high and low emission scenarios are either caused by divergent assumptions about economic and population growth or by assumed changes in energy efficiency and fuel mix or both. Further differences emanate from the assumptions about new technologies, fuel prices and policy measures. 
In De Vries and Janssen (1996), four scenarios are described which have been constructed with the energy model of TARGETS. The aim was to investigate the sets of assumptions underlying published scenarios of the IPCC (1992), Kassler (1995), and $\amalg A S A / W E C ~(1995)$, and to see whether the outcomes as published by the authors of the aforementioned scenarios can be reproduced. The four scenarios, for which the same population and economic growth projections are assumed (Figure $6.1)$, are briefly described here.
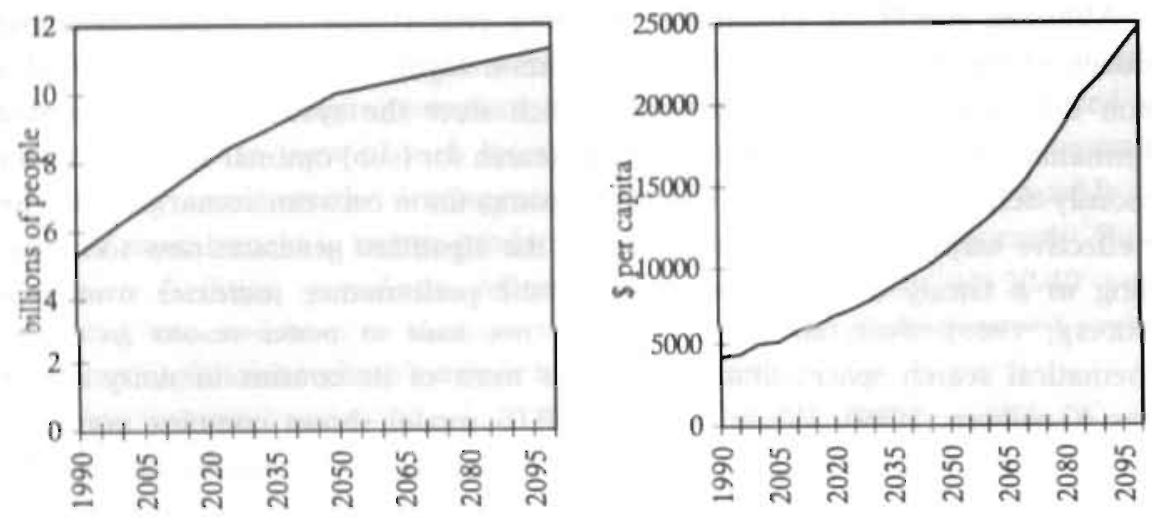

Figure 6.1: Assumptions about the population and the gross world product (GWP) per capita for the period $1990 \cdot 2100$.

The IPCC-IS92a scenario is one of the most widely discussed scenarios in the context of energy and climate, and is constructed by Pepper et al. (1992) for the IPCC Working Group 3. In many studies, it serves as a reference scenario against which other options and developments are judged. Its key features are, from a global longterm perspective (1990-2100), that: (i) GWP grows with an average of $2.3 \%$ a year; (ii) use of secondary fuels and electricity triples; (iii) oil and gas are largely depleted within the next century; (iv) coal is the major substitute; (v) electricity is increasingly generated by non-thermal technologies (NTE), up to $60 \%$ by 2100 ; (vi) biofuels penetrate the market for liquid (BLF) and gaseous (BGF) fuels slowly up to $10 \%$ by 2100. Using the characteristics of the IS92a scenario, assumptions within the energy model are constructed in such a way that it simulates the main aspects of IS92a, which I call Conventional Wisdom scenario (CW) (Figure 6.2). The continued use of fossil fuels in the next decades initially leads to a relative decrease in energy expenditures (Figure 6.6), due to efficiency improvements in the use and production of energy. Given the assumption of the IS92a scenario that oil and gas largely deplete during the next century, the CW scenario shows an increase of energy expenditures as 
a fraction of GWP from 2010 onwards. A difficult transition period follows, in which even more expensive oil and gas are replaced by still costly alternatives (biofuels, NTE). By 2040, this transition is largely over. Energy efficiency improvements lead to a decreasing trend of relative expenditures in the period thereafter. The fossil $\mathrm{CO}_{2}$ emissions in this scenario increase up to about $19 \mathrm{GtC}$ in 2100 (Figure 6.6); the penetration of biofuels leads to a temporarily decrease of global $\mathrm{CO}_{2}$ emission in the middle of the next century. According to simulations with the CYCLES module, the global energy scenario leads to a $\mathrm{CO}_{2}$ concentration increasing to about $750 \mathrm{ppmv}$ in 2100 and a global mean temperature increase of about $2.5^{\circ} \mathrm{C}$ degrees compared to 1900.

The first alternative scenario is based on more optimistic assumptions on technological progress in the supply side of the energy system (Figure 6.3). In this Supply Oriented Technology Change scenario (SOTC), economic growth is the same as in IS92a. Final energy demand closely follows the IS92a scenario, but the supply side differs significantly. New technology will be developed so that non-carbon energy options will become much cheaper and markets will ensure their subsequent introduction. Coal is assumed to be much more expensive, among other things, because subsidies are removed, and coal use is, therefore, much smaller. Because alternatives are introduced in this scenario at lower costs than the $\mathrm{CW}$ scenario, energy expenditures as fraction of GWP will decrease earlier during the transition to alternatives (Figure 6.6). Fossil $\mathrm{CO}_{2}$ emissions steadily decline, leading to a $\mathrm{CO}_{2}$ concentration of $620 \mathrm{ppmv}$ and a temperature increase of $2.3^{\circ} \mathrm{C}$ in 2100 . Due to the reduction of the fossil fuel burning, the cooling effect of sulphate aerosols is lower in the SOTC scenario compared with the CW scenario, leading to a higher increase of the global mean temperature, in the short term.

Using other assumptions on the demand side of the energy system leads to the third scenario: Demand Oriented Technology Change (DOTC) (Figure 6.4). Its key message is that waves of innovative energy efficiency technologies, in combination with shifts in economic activity patterns, make it possible to sustain a 2.3\%/yr GDPgrowth at much lower energy use and, hence, a lower carbon emission path. Estimated final energy demand is much lower than IS92a, but the supply side assumptions are the same as used for the IS92a scenario. Due to a lower energy demand, the pressure on fossil fuel resources is lower, leading to a lower increase of relative energy expenditures (Figure 6.6). In the DOTC scenario, $\mathrm{CO}_{2}$ emission trajectory is until 2080 lower than in the SOTC scenario. After 2080 , the lack of cheap 
alternatives causes an increase of $\mathrm{CO}_{2}$ emissions, leading to a $\mathrm{CO}_{2}$ concentration of 590 ppmv and a temperature increase of $2.1^{\circ} \mathrm{C}$ in 2100 .

Finally, by combining assumptions of SOTC and DOTC scenarios, the fourth scenario is constructed, which I call Energy System Technological Change (ESTC) (Figure 6.5). Energy production stabilizes at $50 \%$ above the present level. Together with the successful introduction of alternatives, fossil fuel use decreases during the next century. Because alternatives can be introduced at low cost and because of large short-term efficiency improvements, relative energy expenditures in this scenario are the lowest of all four scenarios (Figure 6.6). The $\mathrm{CO}_{2}$ concentration stabilizes at 500 ppmv, resulting in a global mean temperature increase of $1.9^{\circ} \mathrm{C}$ in 2100 .

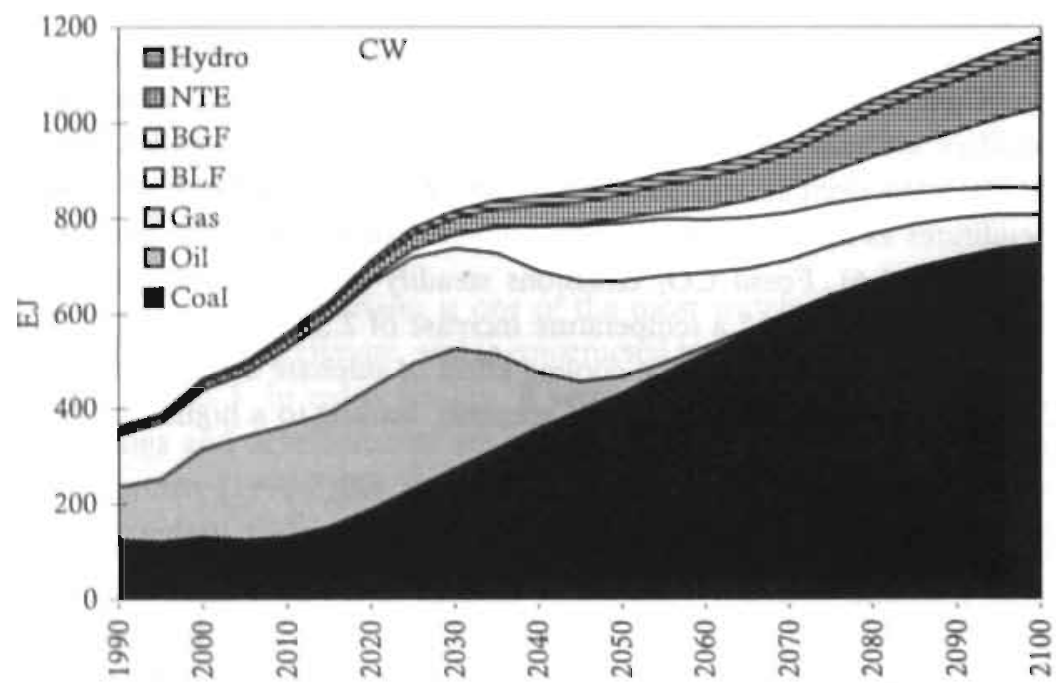

Figure 6.2: Fuel mix of the energy production for the CW scenario. 


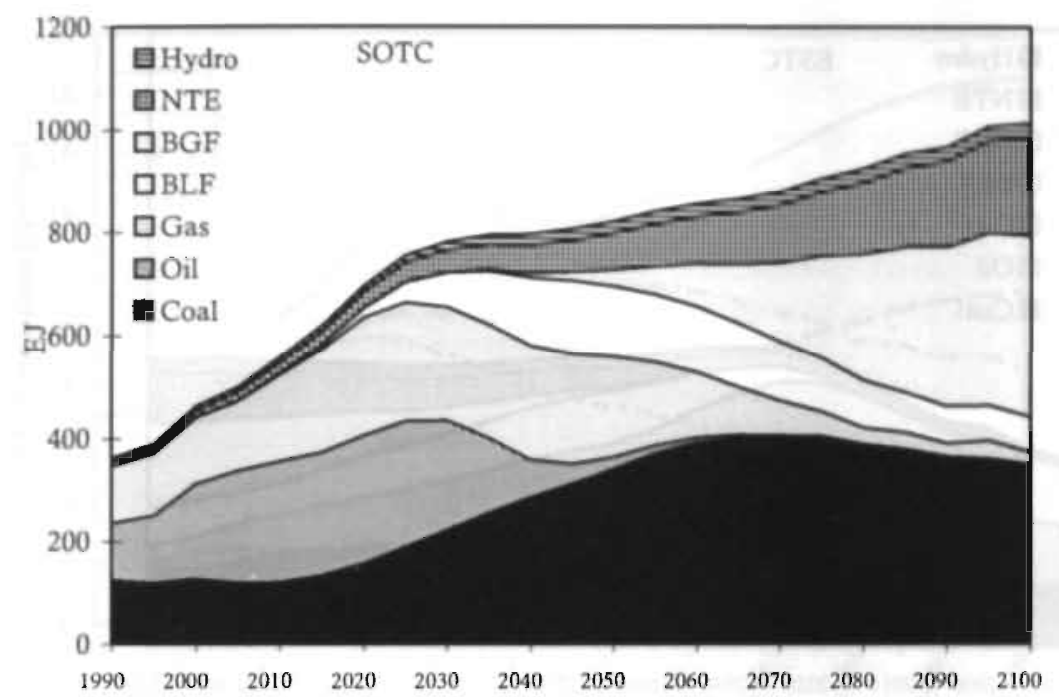

Figure 6.3: Fuel mix of the energy production for the SOTC

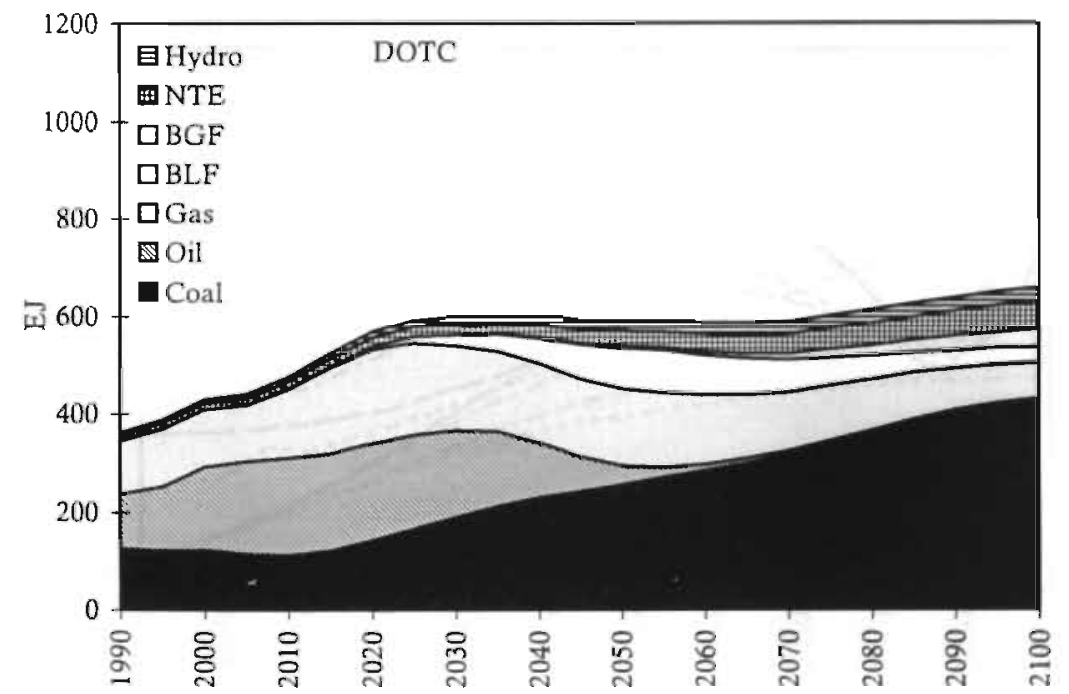

Figure 6.4: Fuel mix of the energy production for the DOTC 


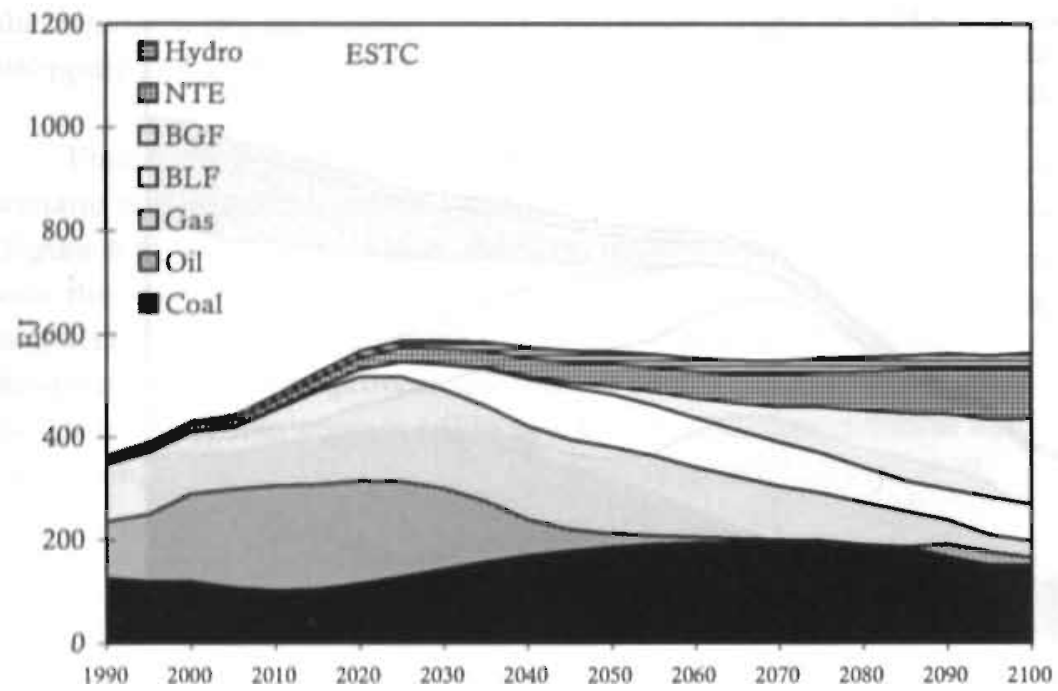

Figure 6.5: Fuel mix of the energy production for the ESTC

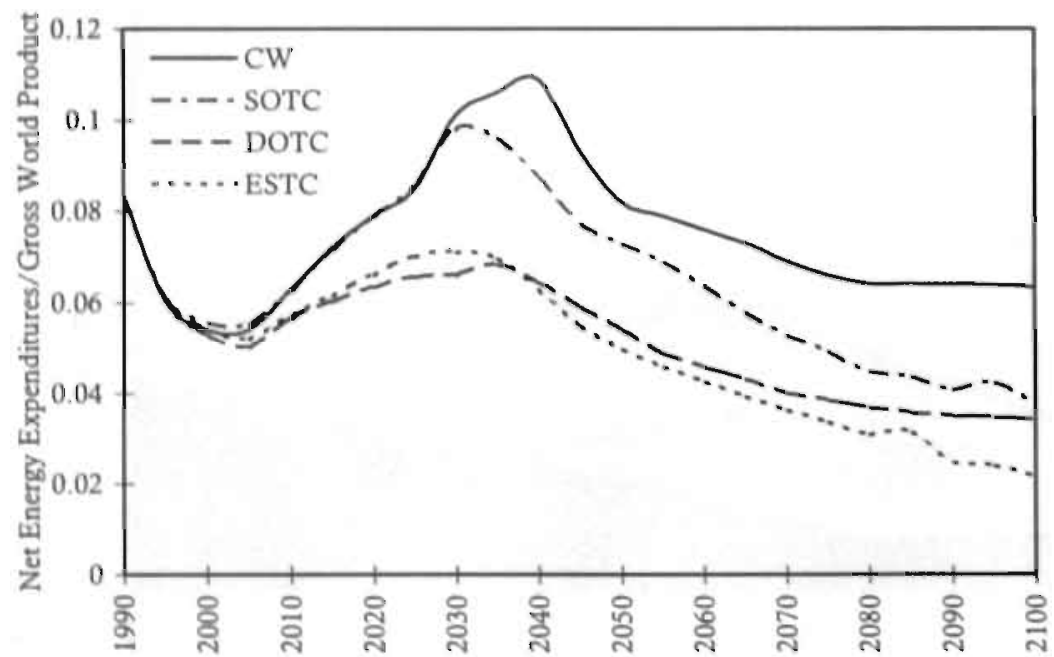

Figure 6.6a: The net expenditures per GWP projections for the four constructed scenarios. 


\section{GLOBAL ENERGY STRATEGIES.}

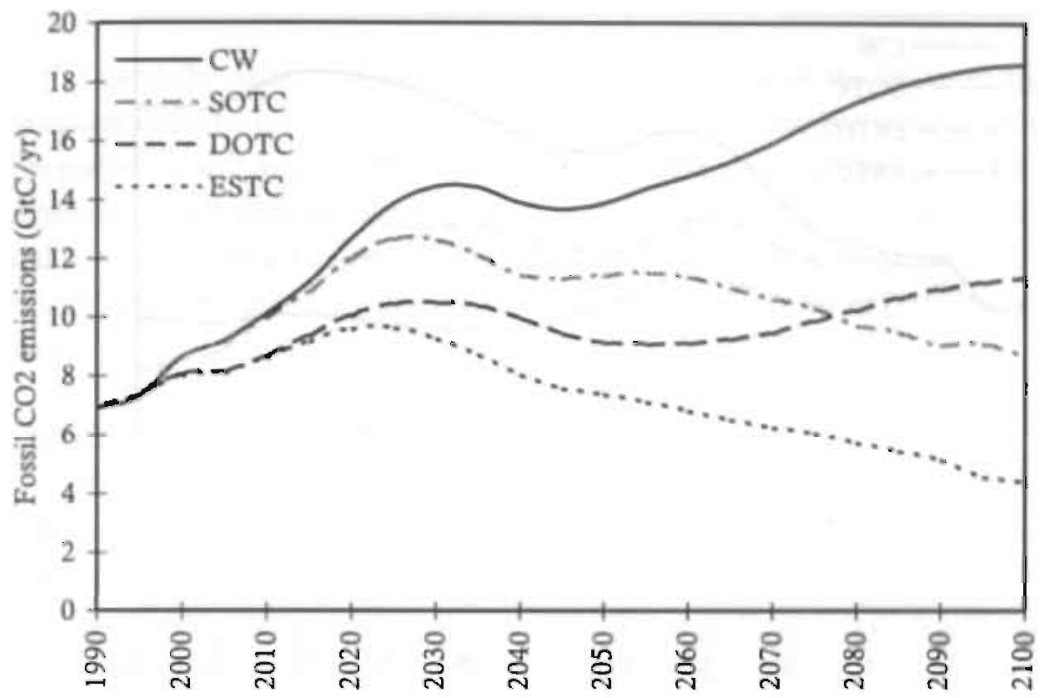

Figure 6.6b: The fossil $\mathrm{CO}_{2}$ emissions projections for the four constructed scenarios.

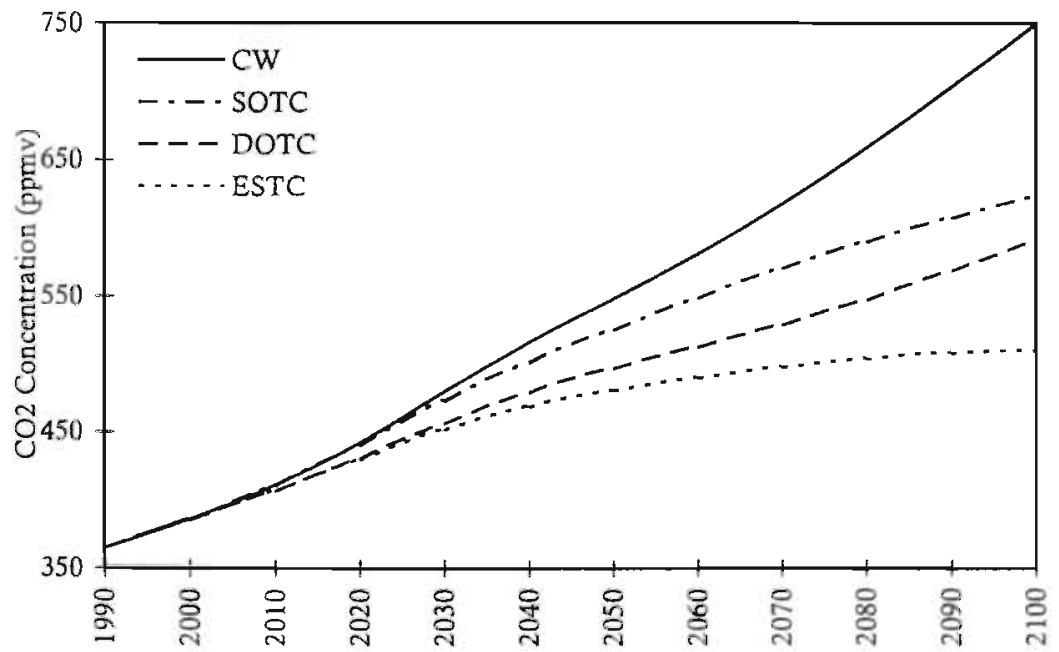

Figure 6.6c: The $\mathrm{CO}_{2}$ concentration projections for the four constructed scenarios. 
MEETING TARGETS

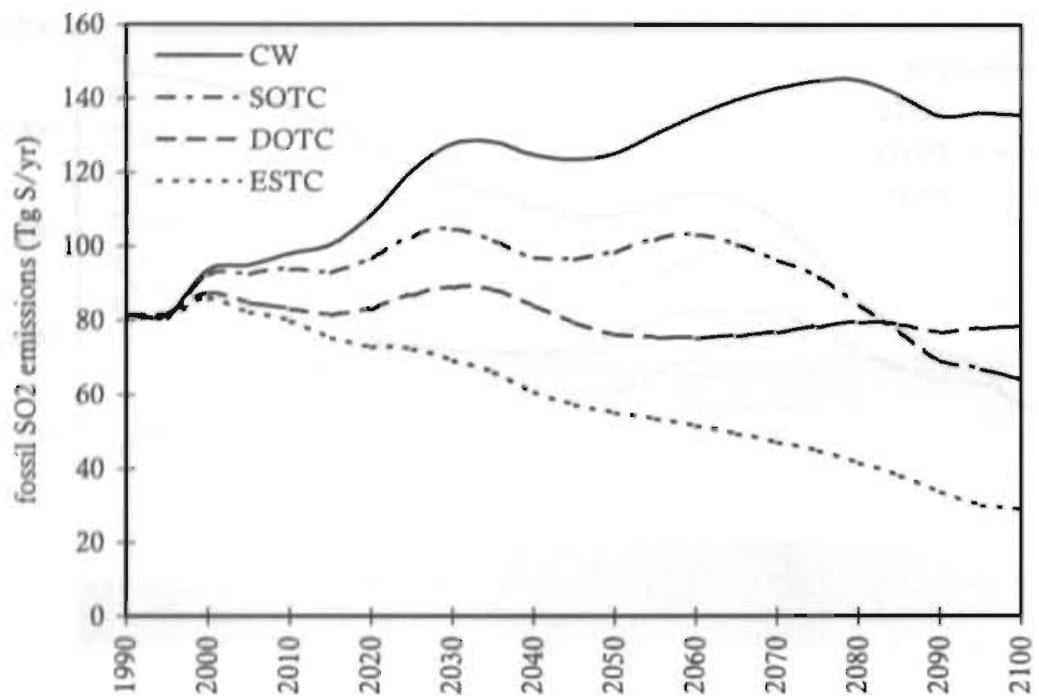

Figure 6.6d: The fossil $\mathrm{SO}_{2}$ emissions projections for the four constructed scenarios.

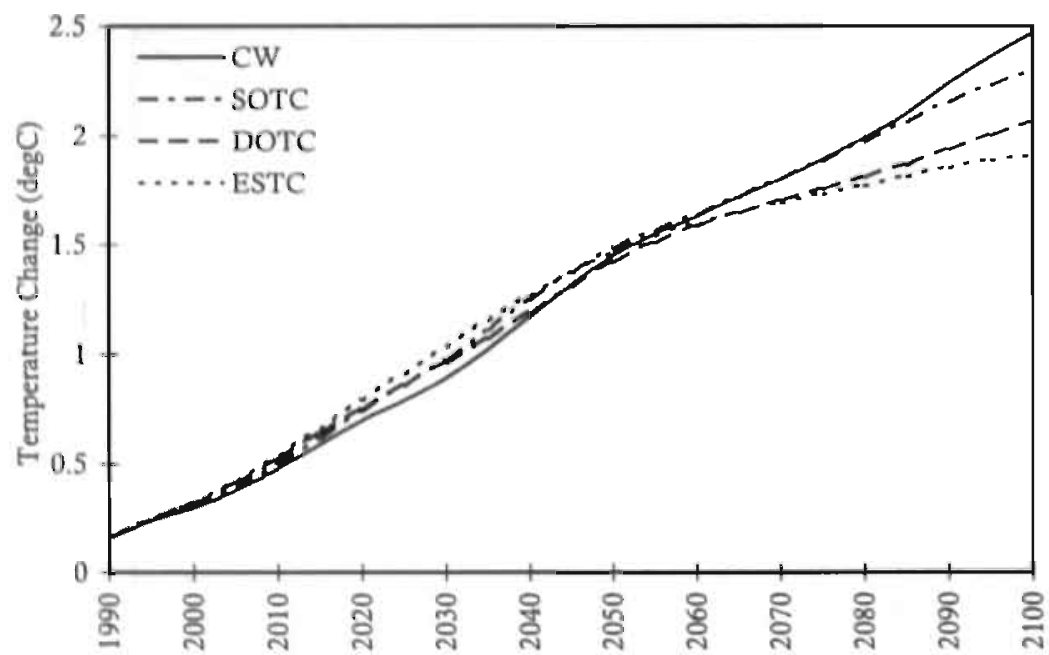

Figure 6.6e: The global mean temperature projections for the four constructed scenarios. 


\subsection{Climate Change Constraints}

I used the assumptions for the $\mathrm{CW}$ scenario to scan the decision space for $\mathrm{CO}_{2}$ emission reduction strategies which meet a range of possible climate change targets. Thereafter, I will analyze the consequences of the uncertainties in technological and economic developments. An "optimal" path is defined as a scenario of R\&D programs and carbon tax which meets the climate change constraints at the lowest possible costs for the next century, using energy expenditures as a fraction of GWP as a measure for costs.

\section{$\mathrm{CO}_{2}$ Concentration Target}

A range of $\mathrm{CO}_{2}$ concentration upper levels (not necessary stabilization) is considered from $700 \mathrm{ppmv}$ to $500 \mathrm{ppmv}$ for the next century. A lower $\mathrm{CO}_{2}$ concentration than 500 ppmv by 2100 was not found in the model exercises. Figure 6.7 a shows the emission paths of the strategies, which do not look like the traditional macro-economic optimal emission paths. The fluctuations of emissions are caused by using a different type of model, where various investment decisions are based on rulebased behavior of agents with a short time horizon. This may result in strong fluctuations in the "optimal" decision variables. An optimal result, therefore, represents the utopian case that the "government" has a perfect knowledge of the system where all private agents (e.g. oil companies) make short time decisions. The optimal policy steers the decisions of the private agents in such a way that the long term objectives of the "government" will be met.

In all cases, an early reduction of emission was found to be efficient. An explanation for this is the fact that an early taxation of fossil fuels to stimulate alternative fuels also reduces the energy demand and stimulates price-induced energy efficiency, which has a long-term effect in a lower energy demand. This conclusion conflicts with the conclusions of Wigley et al. (1996); I use a kind of bottom-up approach excluding the macro-economic feedbacks, while Wigley et al. (1996) base. their conclusion on macro-economic insights, neglecting the insights from bottom-up studies. Note that fossil fuels are not phased-out in any scenario because of the assumption of large inexpensive coal resources, and the assumed slow price induced penetration possibilities of alternative fuels.

The resulting $\mathrm{CO}_{2}$ concentration and global mean temperature projections are depicted in Figures $6.7 \mathrm{~b}+\mathrm{c}$. The reduced warming due to reductions of $\mathrm{CO}_{2}$ emissions is compensated, in global mean terms, by an increased warming due to reductions of $\mathrm{SO}_{2}$ emissions. Therefore, a reduction policy leads to relatively warmer temperatures 
in the coming 50 years, followed by a reduced temperature increase. The transition to a fossil-low energy supply leads to a reduction of 0.7 degree Celsius in 2100 .

The cost-effectiveness of the target strategies is illustrated by plotting the average net energy expenditures as fraction of GWP as a function of the $\mathrm{CO}_{2}$ concentration in 2100 (Figure 6.8). The results suggest that the first steps in reducing the $\mathrm{CO}_{2}$ concentration can be made with relatively few extra investments in agreement with most bottom-up analyses. Meeting the $550 \mathrm{ppmv}$ concentration targets requires in the CW-scenario an increase of $50 \%$ in energy expenditures. In the latter experiment, an immediate carbon tax policy of the fossil energy prices triples in the coming years.

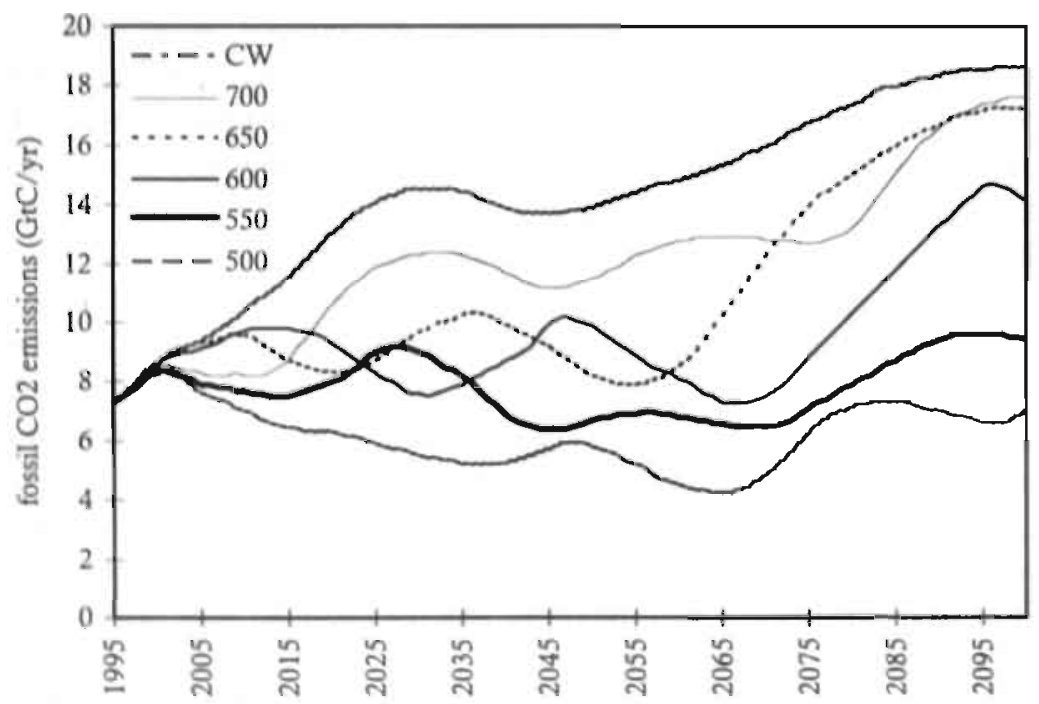

Figure 6.7a: Emission paths of fossil $\mathrm{CO}_{2}$ for strategies meeting the $\mathrm{CO}_{2}$ concentration targets. 


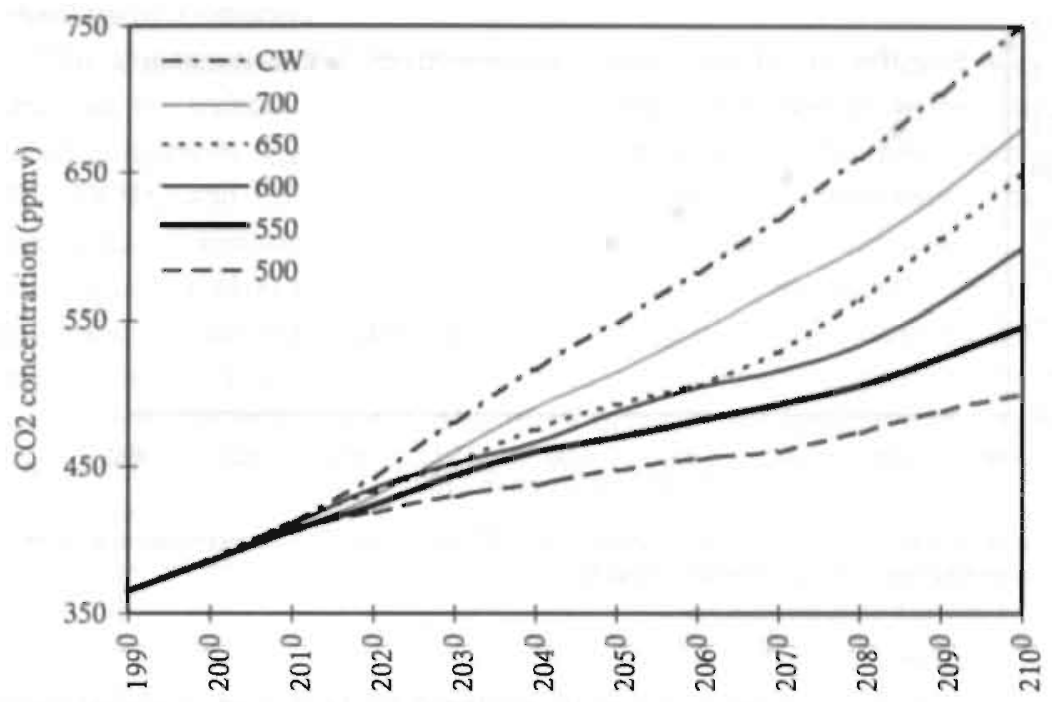

Figure $6.7 \mathrm{~b}$ : $\mathrm{CO}_{2}$ concentration projections for strategies meeting the $\mathrm{CO}_{2}$ concentration targets.

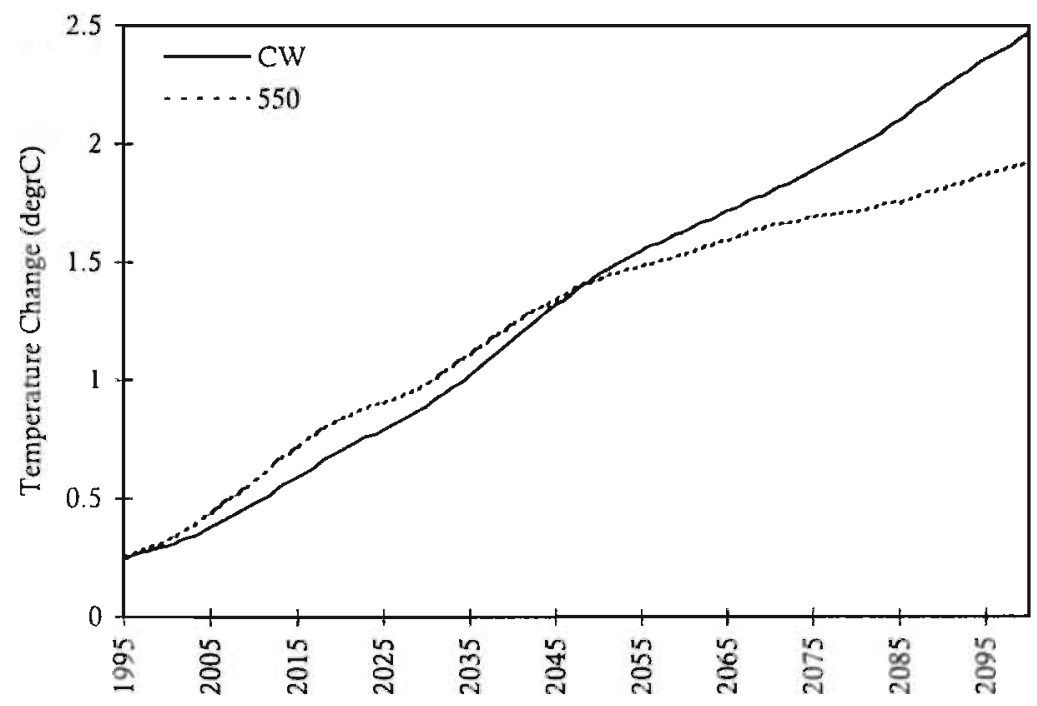

Figure $6.7 \mathrm{c}$ : Global mean temperature change projections for the strategy meeting the 550 ppmy concentration target. 


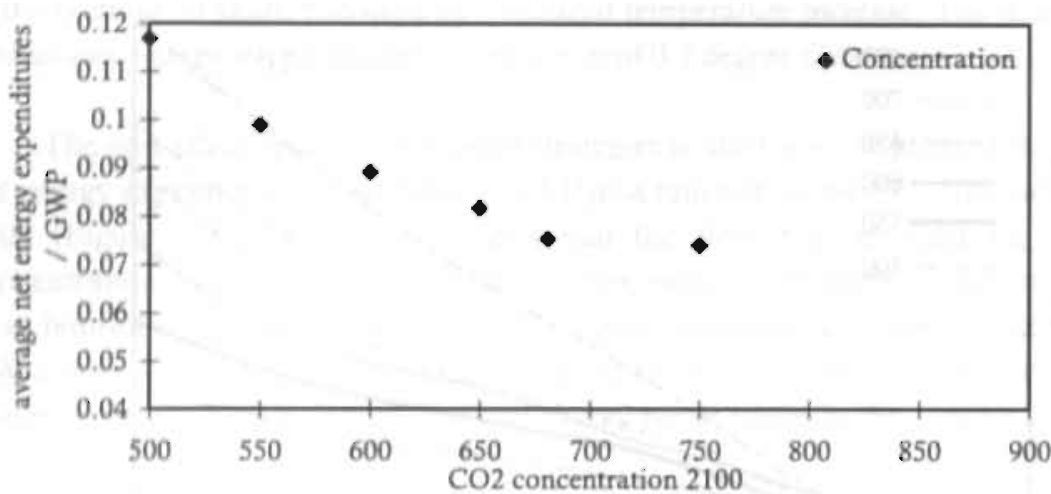

Figure 6.8: Average net energy expenditures divided by GWP versus the $\mathrm{CO}_{2}$ concentration in 2100 for the strategies meeting the $\mathrm{CO}_{2}$ concentration targets.

\section{Temperature Target}

I have also explored emission reduction strategies to meet a set of global mean temperature constraints. Due to the reductions of both cooling and warming effects if fossil fuel use decreases, relative costs rise rather strongly for relatively small reductions of the global mean temperature increase (Figure 6.9). Due to the dependence on $\mathrm{SO}_{2}$ reduction policies, which are taken to be exogenous, this result is highly uncertain (see also Section 6.8).

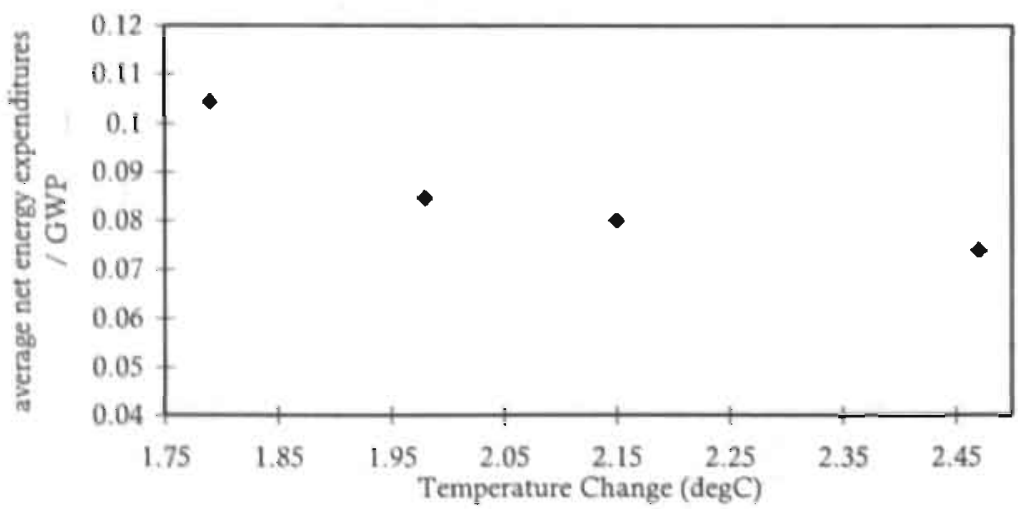

Figure 6.9: Average net energy expenditures divided by GWP versus the global mean temperature in 2100 for the strategies meeting the global mean temperature targets. 


\section{Technological Transition}

The abilities to meet environmental constraints in an efficient way greatly depends on the technological development of the energy system, as discussed in the previous paragraphs. Figure 6.10 illustrates this by including the three other scenarios SOTC, DOTC and ESTC within the cost-effectiveness representation. The kind of technological transitions assumed for these scenarios could reduce the $\mathrm{CO}_{2}$ concentrations in 2100 at much lower relative costs. Still, meeting the $550 \mathrm{ppmv} \mathrm{CO}_{2}$ concentration constraint requires additional policy measures except for ESTC. The probability of a technological transition is an important element for energy policy in the short-term, an issue discussed in more detail in the coming paragraphs. First, another highly uncertain and important energy policy issue is discussed: long term economic growth.

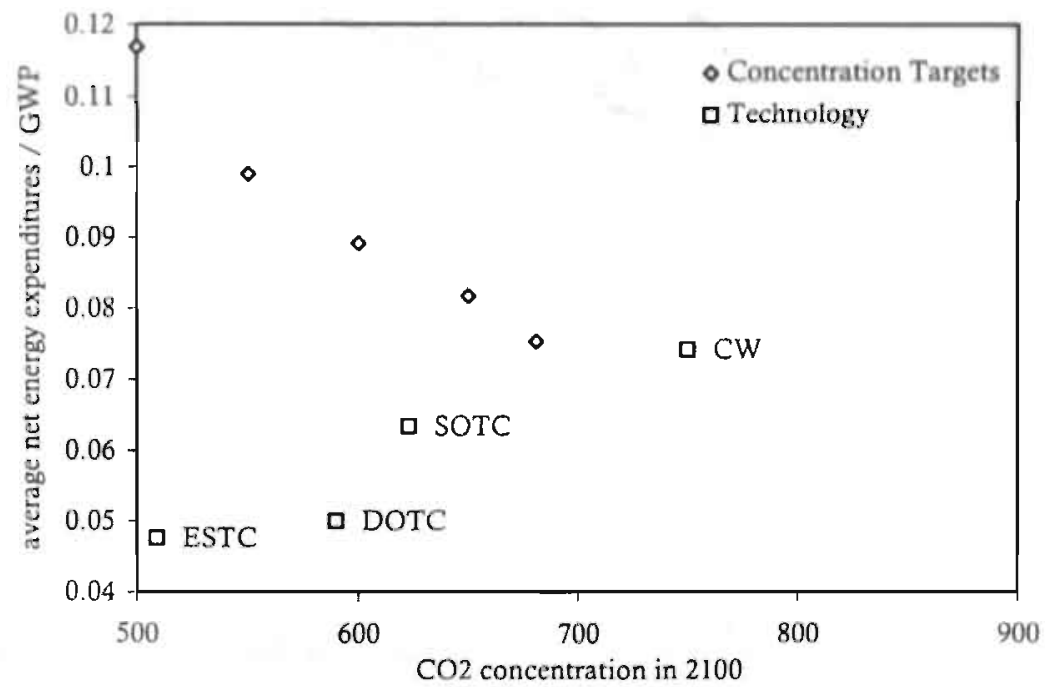

Figure 6.10: As Figure 6.9 plus three scenarios which include a technological shift.

\section{Economic Growth}

In the four scenarios, an average $2.3 \% / \mathrm{yr}$ GWP growth between 1990 and 2100 is assumed. An annual growth rate of $3.0 \% / \mathrm{yr}$ would lead to an average GWP per capita of about 50.000 US $90 \$$ in 2100 , about twice as high as in the CW scenario. The resulting increase in the use of fossil fuels leads to $\mathrm{CO}_{2}$ emissions of $35 \mathrm{GrC}$ in 2100 . Because of high $\mathrm{SO}_{2}$ emissions, the projected global mean temperature increase is only slightly higher. The impact on energy expenditures is shown in Figure 6.11. Higher energy demand leads to higher use of fossil fuels and higher energy prices, 
which is only partly offset by more energy conservation and greater penetration of renewable energy sources. Hence, higher economic growth reduces the impact of technology improvement on the $\mathrm{CO}_{2}$ emission reduction goals.

Because the interactions between energy policy, economic developments, and technological innovation are not modelled, it is speculative to discuss the consequences for abatement in greater detail.

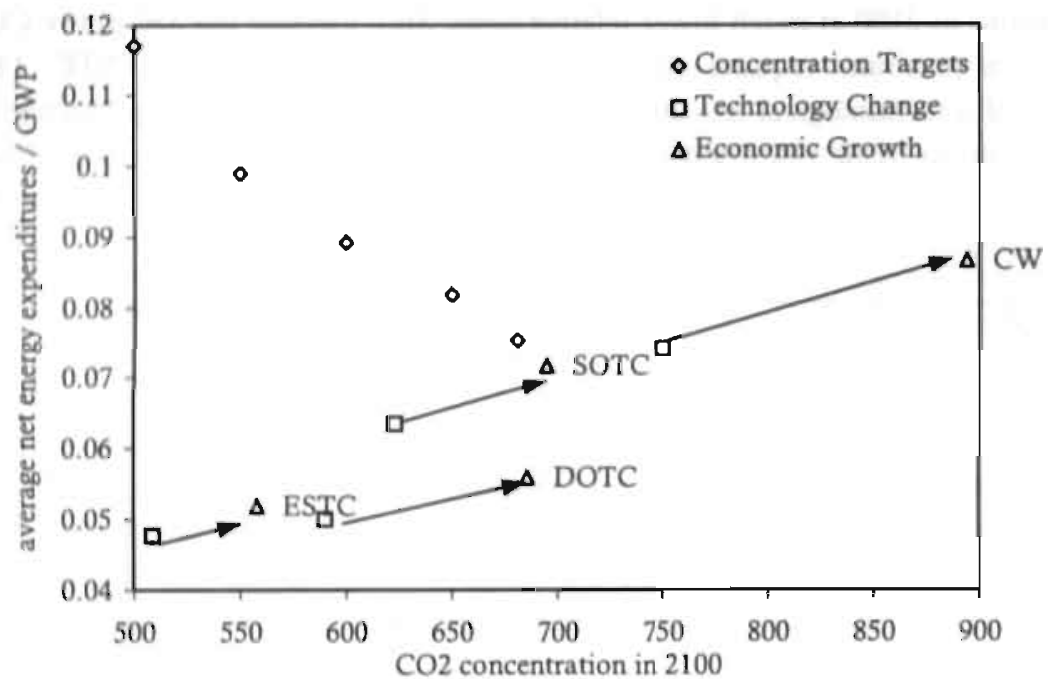

Figure 6.11: As Figure 6.10 plus four scenarios for which a higher economic growth rate is assumed (=annual average of $3.0 \%$ ).

\subsection{Delayed Response}

The large uncertainties in economic and technological developments and the large sensitivity of the "optimal" response in order to meet climate change targets determine the effectiveness and costs of policy options in the short term. What are the risks of implementing abatement policies at a later date? As an illustration, a delayed response strategy is explored in which no active energy policy is assumed to be implemented until 2010. The CW scenario is used to look for an optimal strategy thereafter to meet the $550 \mathrm{ppmv}$ constraint. That is to say that governments initially assume a low impact of emissions on the climate system and/or a rapid technological transition to a non-fossil energy system. After 15 years (1995-2010), a change in insights and facts leads to a new energy policy. In the TARGETS model, such a delayed response leads to higher costs (Figure 6.12 (wait and see); Figure 6.14) 
because of a rapid and perhaps drastic emission abatement policy just after 2010, in order to meet the $\mathrm{CO}_{2}$ concentration targets. The consequences in terms of global mean temperature change are an accelerated increase from 2010 onwards (Figure 6.13). If the rate of temperature change is viewed rightly as one of the indicators of climate change related risks (Alcamo and Kreileman, 1996), the delayed response strategy of "wait-and-see" is probably a risky one (Figure 6.15).

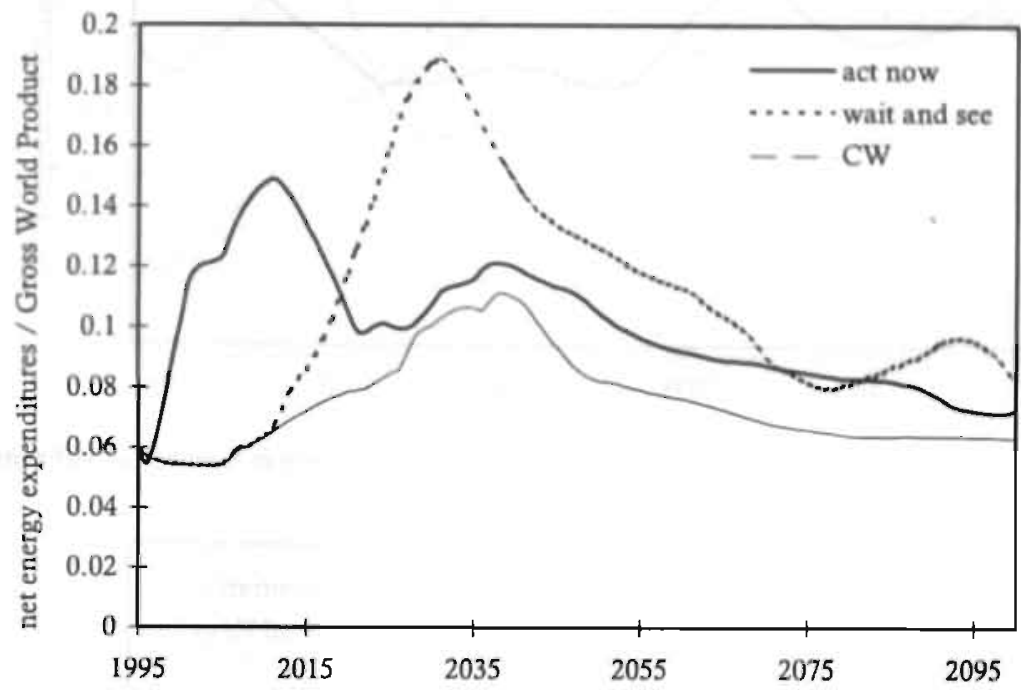

Figure 6.12: The net energy expenditures per GWP for an optimal path and a delayed response in meeting the 550 ppmv target. 


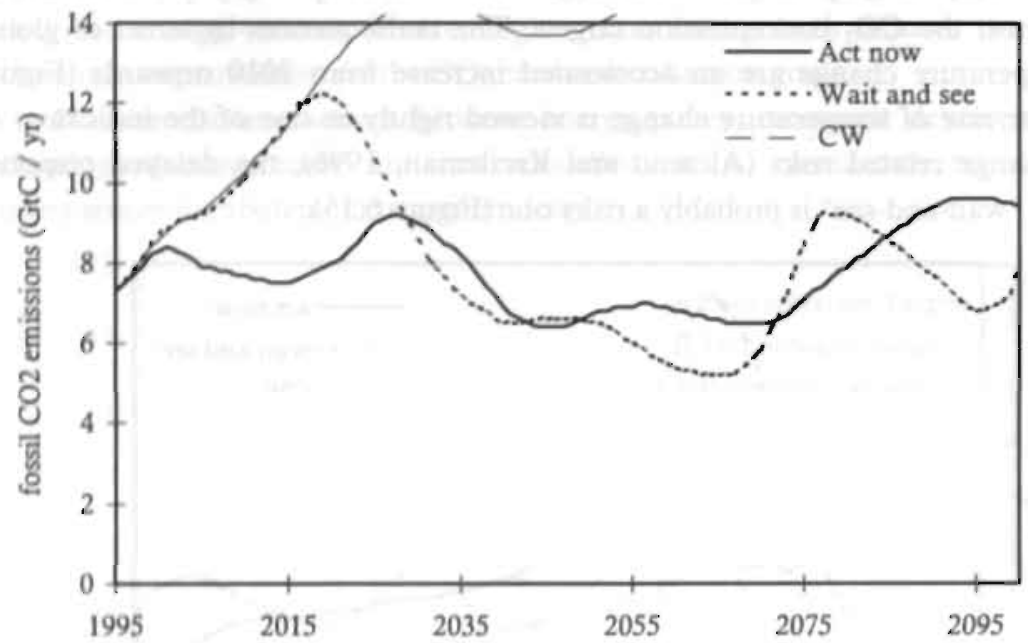

Figure 6.13: Fossil $\mathrm{CO}_{2}$ emissions for an optimal path and a delayed response in meeting the 550 ppmv targets.

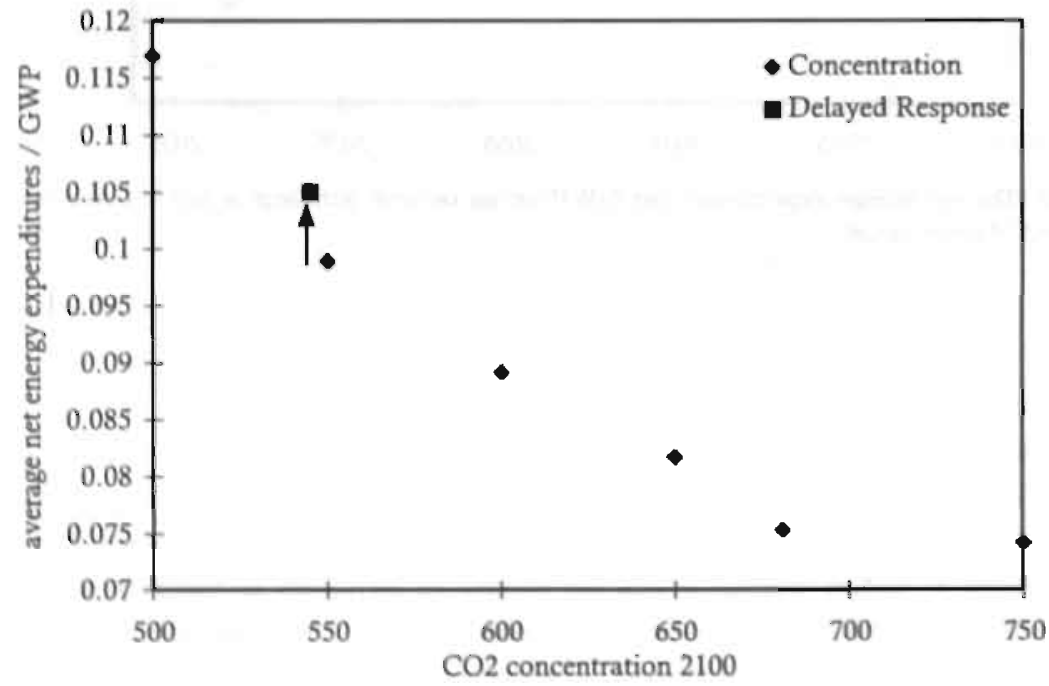

Figure 6.14: The delayed response leads to a higher level of the average net energy expenditures per GWP. 


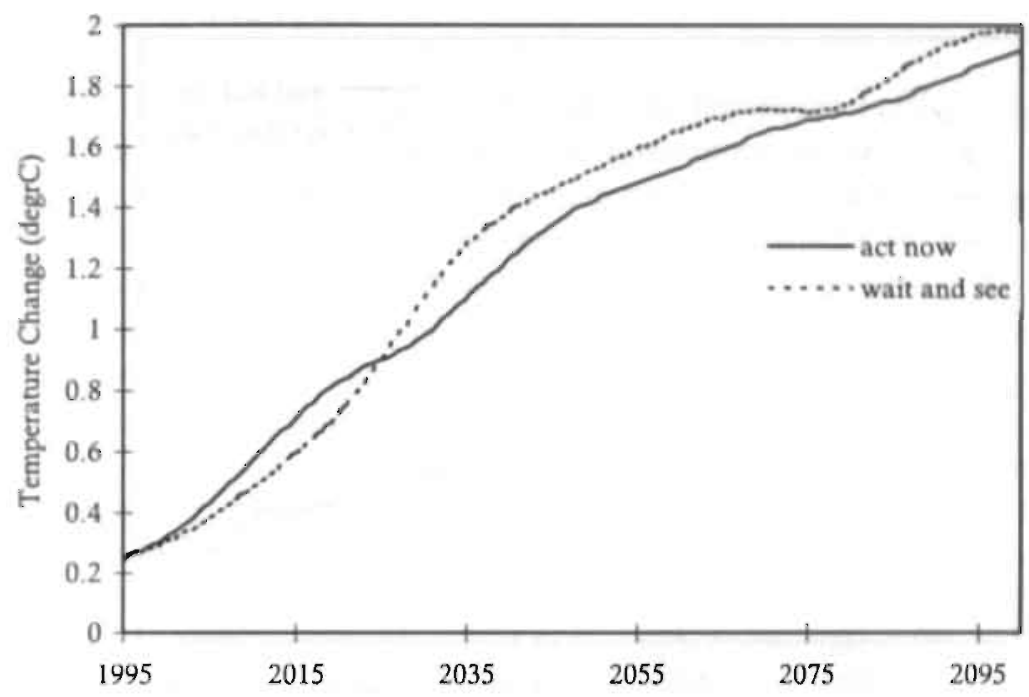

Figure 6.15: The delayed response leads to a fast increase in the global mean temperature when the policy is implemented.

Next, the reverse situation is examined: implementing strong energy policies which turn out to be unnecessary. Using ESTC scenario assumptions, what are the consequences of an active climate change policy, which is then terminated in 2010 ?

In the first decades, the relative net energy expenditures increase sharply if the carbon tax and R\&D policies are implemented; they fall when they are terminated (Figure 6.16 (act now ESTC)). However, the price-induced efficiency improvements in the first decades induce large energy efficiency improvements, which leads to lower relative expenditures after 2020 than in the ESTC scenario without policy measures. As a result, the aggregated net energy expenditures over the period 1995-2100 are lower in the case of early intervention than without. It is not surprising that the $\mathrm{CO}_{2}$ emissions are lower in case of a short period of active energy policy (Figure 6.17). However, given reference assumptions on the climate system, a no-policy scenario was already sufficient to meet frequently used climate targets (see Figures 6.6). Early emission reduction measures are found to be efficient because early taxation of fossil. fuels not only stimulates alternatives, but also stimulates price-induced energy conservation investments. 


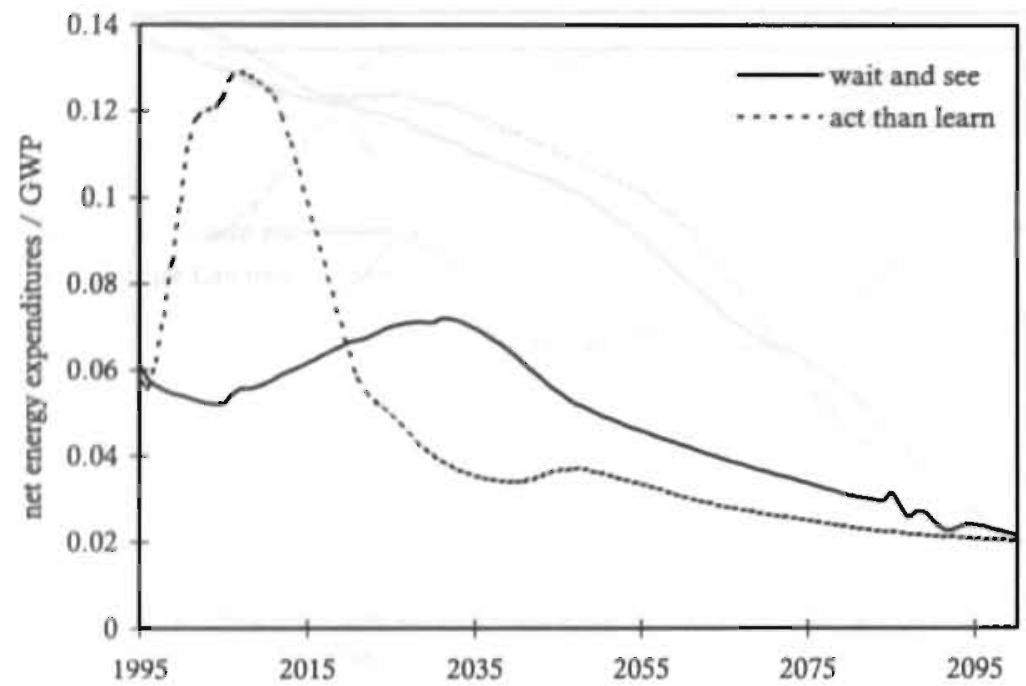

Figure 6.16: The net energy expenditures per GWP for an optimal, path and a delayed, response when a technological transition occur.

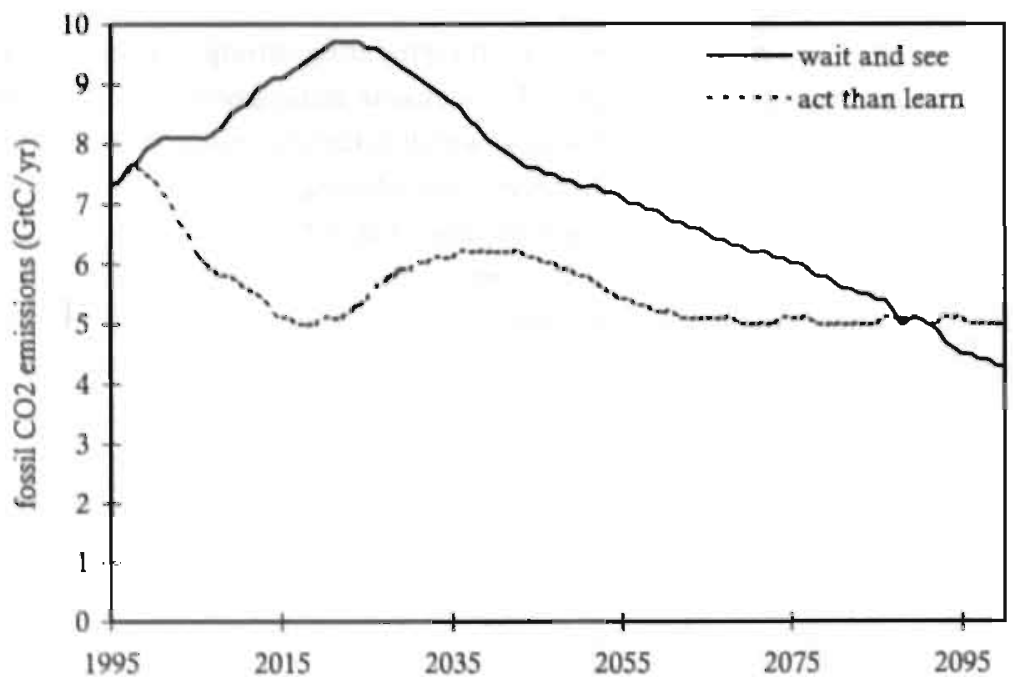

Figure 6.17: Fossil $\mathrm{CO}_{2}$ emissions for an optimal path and a delayed response when a technological transition occurs. 


\subsection{Hedging Strategies}

The above exercises illustrate the important consequences of first (not) act and then learn. Whether or not technological innovations are accelerated, it makes a difference in determining cost-effective strategies. In this Section, I will focus on the short term policy (until 2010), assuming perfect knowledge of the technological developments in the period thereafter. So, whether a technological progress is accelerated or not, short-term policy is required, given expectations on the likelihood of accelerated technological progress.

Manne and Richels (1992) introduced the concept of hedging as a method to deal with uncertainty in decision-making. Instead of assuming perfect knowledge of the system over the coming century, a period of learning is proposed. In the used optimization framework, this means that the objective function $(1-\alpha) \mathrm{f}_{\mathrm{CW}}+\alpha f_{\mathrm{ESTC}}$ is optimized where $\alpha$ is the subjective probability, whether technological developments evolve along ESTC assumption or not (assuming a Conventional Wisdom Scenario). In fact, the decision variables for the two model versions are optimized, given the restriction that the decision variables have the same values until 2010, and may differ thereafter.

The illustrative experiment assumes a $50 \%$ chance on accelerated technological development as in the ESTC scenario and $50 \%$ chance on technological development according to the Conventional Wisdom scenario. Carbon tax policy is the dominating factor in reducing emissions. The global level of the model makes me careful in making explicit statement about carbon tax levels. Nevertheless, in Figure 6.18 the carbon tax levels of the hedging exercise are depicted to illustrate the concept and the different kind of results compared with top-down models. The carbon tax junps to high levels at the start of the period. An illustrative nice increasing carbon tax scenario in line with a top-down approach is depicted in the same figure. The difference between the approaches is caused by the fact that the system dynamics approach as adopted in the TARGETS model assumes a current state of the energy system which is not necessary optimal in terms of costs. An additional policy may, therefore, lead to a more efficient energy system and lower costs. In the optimization models adopted by the top-down approach, the current state of the energy system is optimal in terns of cost; and any additional policy (like a carbon tax) will lead to extra costs. Therefore, a smoothly increasing tax level path is found to be optimal in those models. 


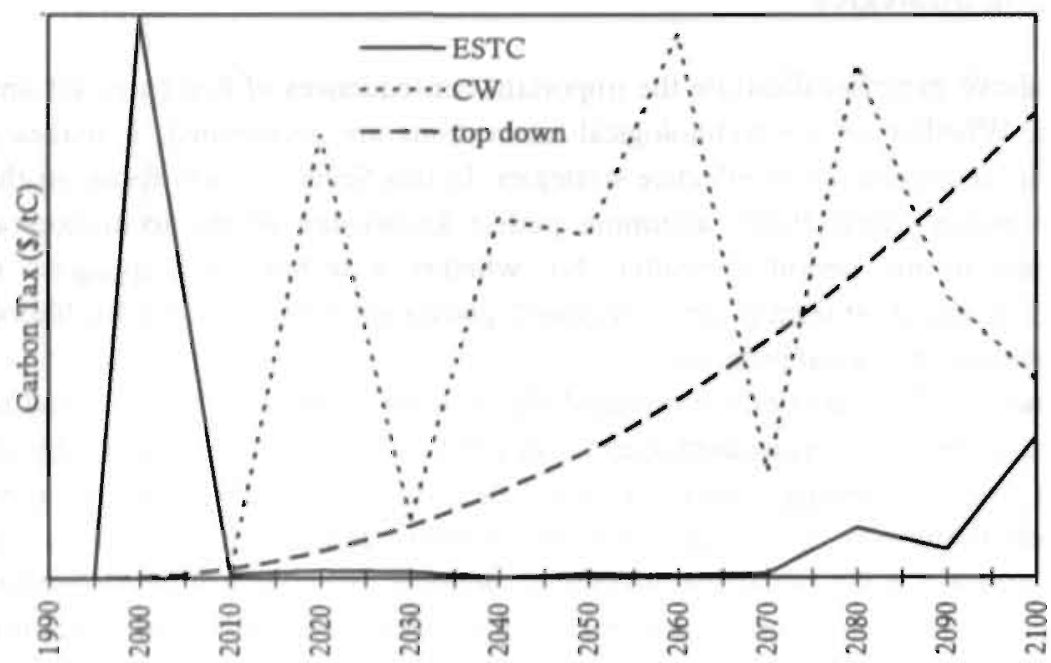

Figure 6.18: Carbon tax levels for the hedging strategy when after 2010 an optimal level is derived according to the technological developments, and a hypothetical carbon tax path for a traditional top. down approach.

In Figure 6.19, the relative changes, compared with the reference, in the energy mix are shown for both the $\mathrm{CW}$ and the ESTC scenario, given a concentration target of 550 ppmv. While the short-term policy for both the CW and the ESTC scenario are the same in the hedging exercise, relative changes do not significantly differ. Due to the fact that carbon intensive energy carriers become more expensive (due to a carbon $\operatorname{tax}$ ), there will be a reduction of coal, oil and gas. However, because of the inertia of the system, oil will first be substituted for coal, before oil will be replaced by bioliquids. Therefore, the oil use will first increase by $20 \mathrm{EJ}$, compared to the $\mathrm{CW}$ and ESTC scenario (current use about $130 \mathrm{EJ}$ ), while the reduction of coal is about $30 \mathrm{EJ}$ compared to the CW and ESTC scenario (current use about 100 EJ). Once the extra production of bioliquids reach levels of $30 \mathrm{EJ}$, the use of oil is decreased with about 70 EJ compared to the CW and ESTC scenario. The overall energy use declines relative to the CW and ESTC scenario, due to price induced efficiency improvements. 


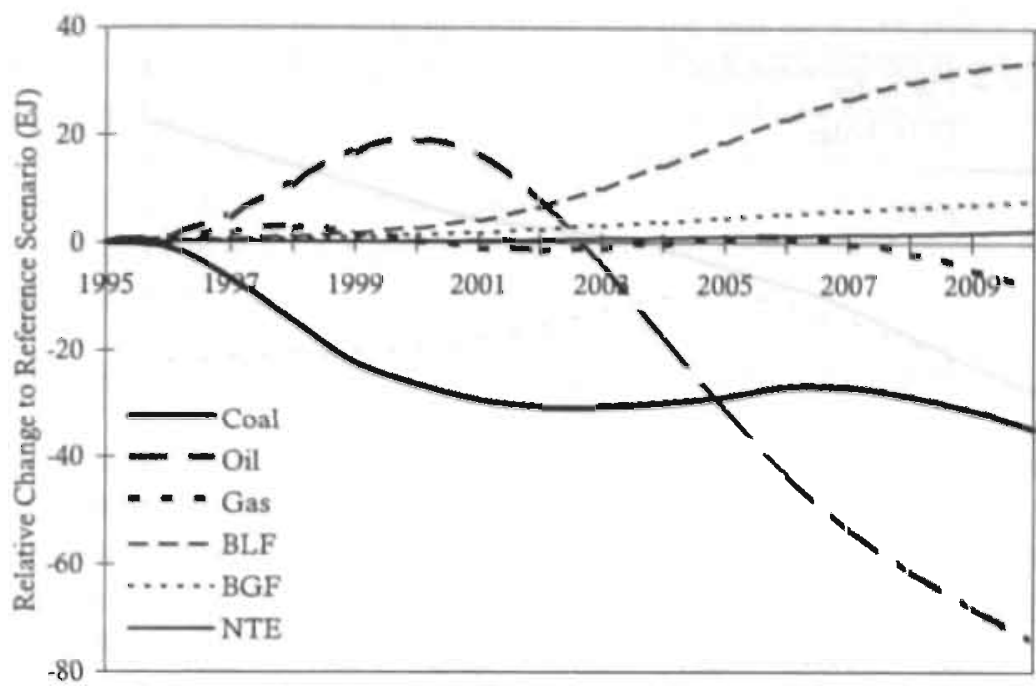

Figure 6.19: Relative change of the fuel mix compared to the reference scenario in case of hedging uncertainties in technological development.

The emission profiles of the hedging strategy are depicted in Figure 6.20, where the impacts on both CW and ESTC are illustrated. The relative reduction to the "nopolicy" scenario is about the same in both cases of technological development. However, due to a fast technological development, emissions can be reduced compared to the 1995 levels, while in the case of CW assumptions, it is less likely. The relative energy expenditures (Figure 6.21) are only a little bit lower, in case of a fast technological development due to a lower energy use.

Not shown in the Figures are the pathways after 2010, where an active policy will be required to meet the $550 \mathrm{ppmv}$ concentration target, if the technological development follows the CW assumptions, while no active policy is necessary, if technological development is fast (ESTC).

The influence of uncertainty on the technological development-paths of the scenarios is analyzed by optimizing hedging strategies for a number of different uncertainty estimates on technological transition $(\alpha)$. The results show that the uncertainty in technological development does not bave a large influence on the active policy. Only when it is certain that a technological transition does not occur $(\alpha=0)$, the energy policy is on an intensified level in 2010, leading to relative high costs. Given the exercises discussed in Section 6.6, these findings are not surprising. 


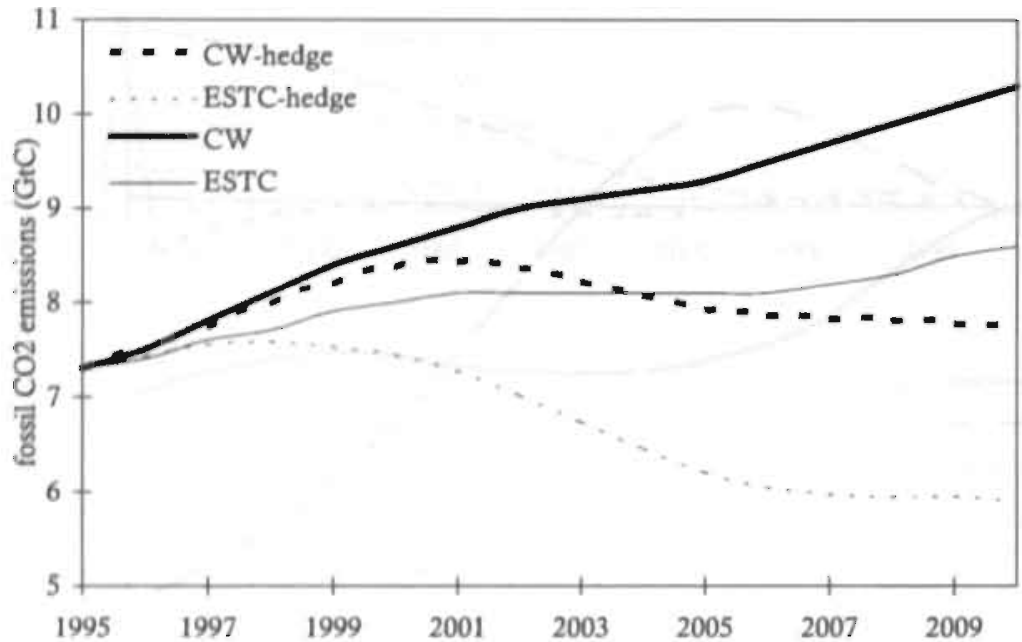

Figure 6.20: The impact of the hedging strategy on fossil $\mathrm{CO}_{2}$ emissions for the two considered scenarios.

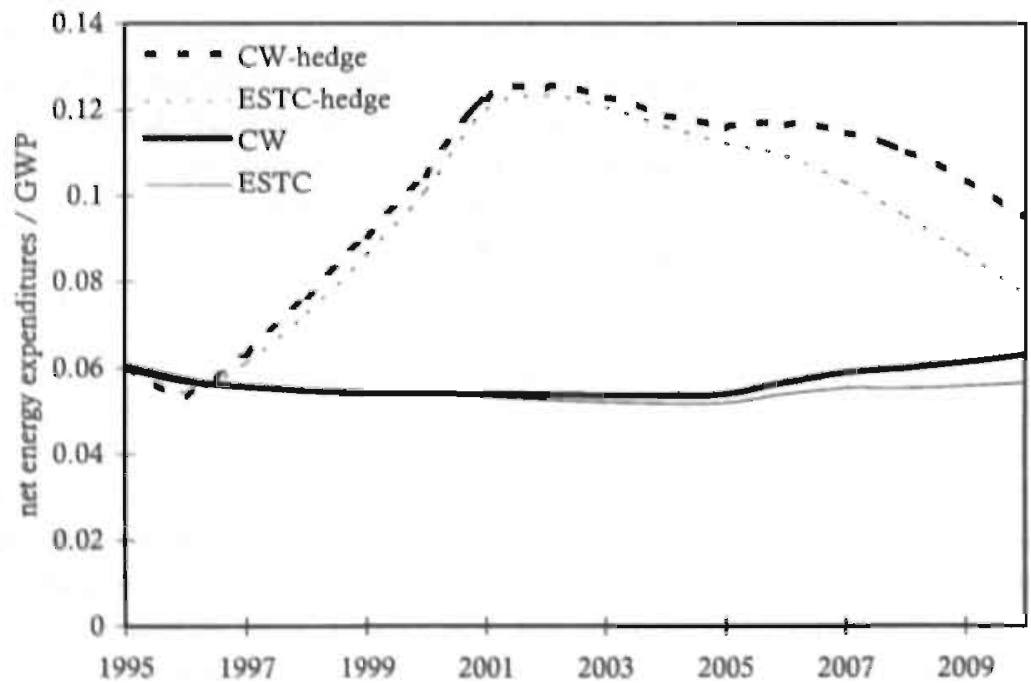

Figure 6.21: The impact of the hedging strategy on net relative energy expenditures for the two considered scenarios. 
To summarize, the hedging exercise tells me that an active policy in the short term has benefits for the long term, irrespective of technological developments. This is caused by the fact that efficiency improvements realized now have their benefits in the decades thereafter, given an unchanged economic development. This last remark, however, denotes an important omission of this study, while it does not incorporate impacts on economic development of technological development, energy policies and climate change.

\subsection{Integrated Policy for Climate Change and Acidification}

The recent quantification that sulphate aerosols have, in global mean terms, a cooling effect has complicated the climate change debate. Because $\mathrm{SO}_{2}$ emissions are also an important contributor to acid deposition at the regional level, it is expected that $\mathrm{SO}_{2}$ specific emission reduction measures will be implemented. This reduction may enhance the expected temperature increase, so that an integrated analysis is required for both $\mathrm{CO}_{2}$ and $\mathrm{SO}_{2}$ emission reduction strategies. Ideally, the local impacts of climate change and acidification should be taken into account. Here ${ }_{\text {I }}$ only illustrate likely trade-offs between the two problems. Recent studies of Alcamo et al. (1995) and Posch et al. (1996) linked the integrated assessment model for climate change IMAGE 2 (Alcamo et al., 1994) and the integrated assessment model for acid rain RAINS (Alcamo et al., 1990; Foell et al., 1995) to assess the combined impact of sulphate policy on ecosystems in Europe and Asia. In their analysis, they conclude that in Europe the impact of sulphate reduction policies benefits ecosystems more than it harms them, while for Asia there is no clear best policy.

The analysis with TARGETS focuses on the fossil fuel transition and the impact. of technological change. Given different futures of the energy system, trade-offs between acid rain and climate change can be identified. The $\mathrm{SO}_{2}$ emissions of the IS92a scenario (IPCC, 1992) are simulated by adjusting the emissions per Joule of fossil fuel (Figure 6.22; reference). Two alternative $\mathrm{SO}_{2}$-specific policies are formulated in relation to acidification policy: an acceleration and a delay of $\mathrm{SO}_{2}$ reduction per Joule of fossil fuels. If no technological transition occurs and no active, policy is implemented, both the problems of acidification and climate change intensify (Figure 6.23). All three scenarios lead to higher risks for the world to suffer from the impacts of acidification and climate change, if one consider the risks to be proportional to global projections. 


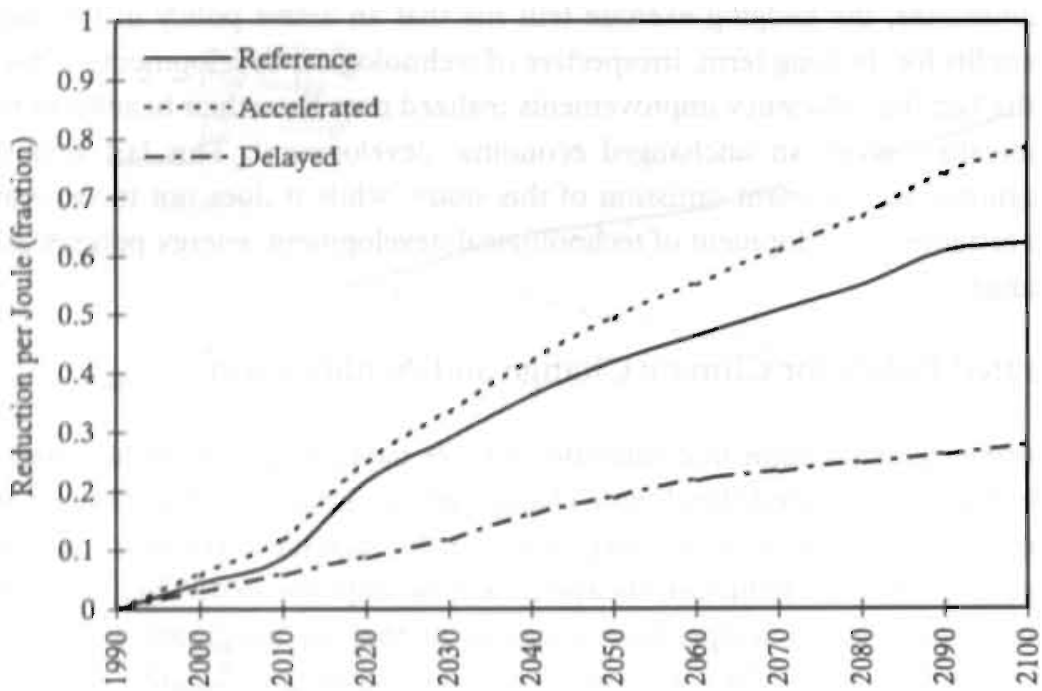

Figure 6.22: Reduction fractions of $\mathrm{SO}_{2}$ emissions per Joule of fossil fuel relative to 1990. The reference case meets the IS92a emission scenario, while accelerated and delayed policies represent altemative $\mathrm{SO}_{2}$ policies.

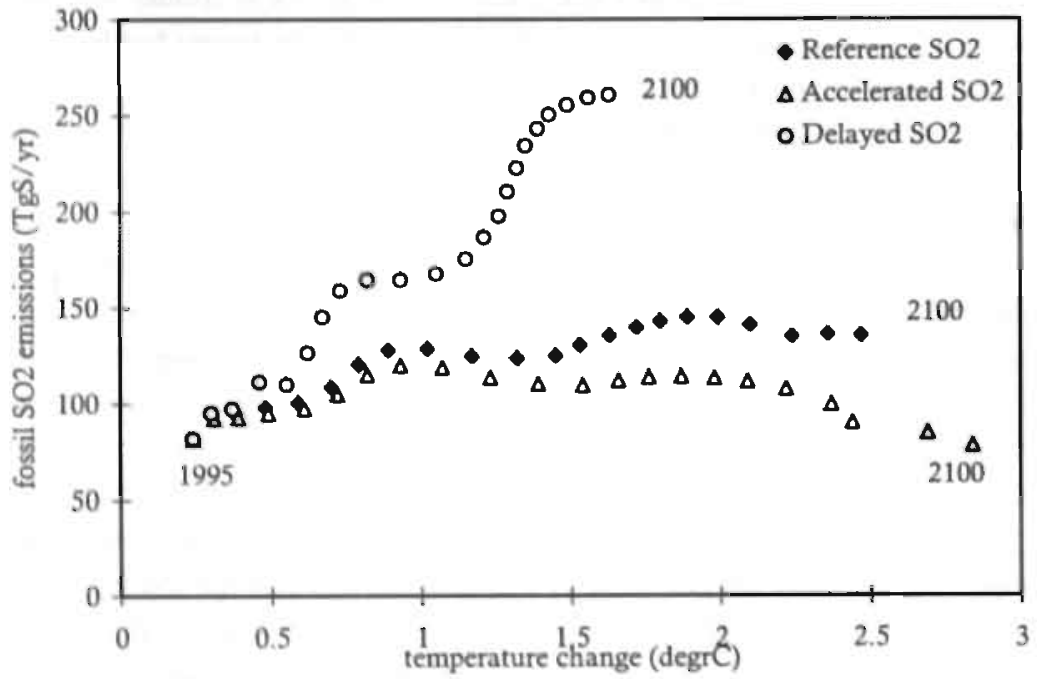

Figure 6.23: The indicators of global. $\mathrm{SO}_{2}$ emissions and global mean temperature change are used to illustrate trade-offs between, acidification and climate change policies in relation to energy use. Given the $\mathrm{CW}$ scenario, a delay of $\mathrm{SO}_{2}$ policy leads to a lower global mean temperature increase. 


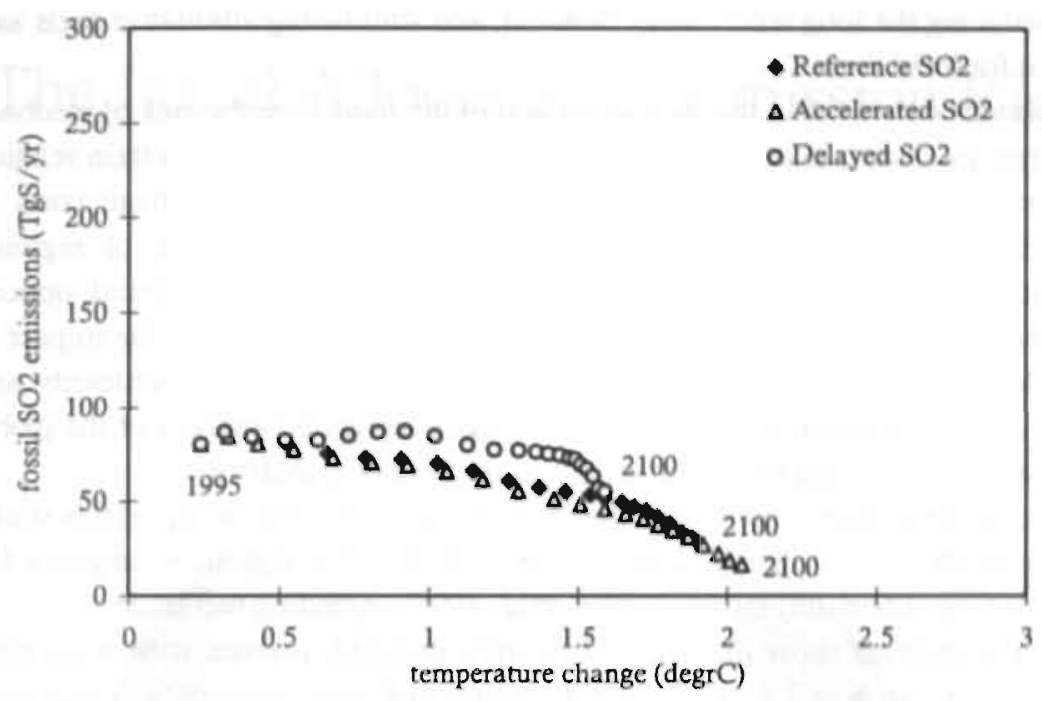

Figure 6.24: As Figure 6.23 where a technical transition according to the ESTC scenario is assumed.

In the previous paragraphs, the use of fossil fuels may decline in the coming century, due to environmental policies, accelerated technological innovations or delayed economic growth.

In Figure 6.24, the technological transition scenario (ESTC) gives an illustration of the integrated impact of reduced fossil fuel use. The $\mathrm{SO}_{2}$ emissions decline for the three $\mathrm{SO}_{2}$ policies, due to an overall reduction in fossil fuel use. However, the projected global mean temperature change is not significantly lower (Figure 6.24). Thus, climate change impacts remain close to the higher risk area, while the impacts of acidification drop to a lower risk area.

\subsection{Conclusions}

The simulation experiments with the Energy-Cycles part of the TARGETS model as presented in this Chapter explore ways to meet climate changes targets and the role of technological change in the energy system. Given conventional wisdom assumptions on technological developments within the energy system, early action is cost-effective to meet long term climate change policy targets. Even with an accelerated technological change in the energy system, early action is found to be cast effective, in the long run, while an optimal functioning energy system was not assumed for the present situation. Such early action may accelerate the energy savings 
options, reducing the long term energy demand, and stimulating alternative fuels as a competitor for fossil fuels.

Important omissions in the current version of the model are the lack of feedback from the energy system to economic growth projections, the highly uncertain relation between economic growth and technological development, the damage costs of climate change impacts and their effect on the economy, absence of regional disaggregation, exclusion of a number of potentially important technical options (among them electric cars, and coal liquefaction and gasification), and the impact of required land for biomass on food production. The performed experiments are, therefore, a first step towards a more comprehensive integrated analysis of the global energy system and its (long term) consequences on the global system.

Some of these shortcomings will diminish, because of a follow- up of this study; the energy model will be disaggregated in the 13 IMAGE 2 regions to improve the IMAGE 2 energy (demand) model (Bollen et al., 1996), including fuel trade.

The experiments show that an optimization oriented analysis with a complex simulation model such as TARGETS can be performed. Such an approach may help to fill the gap between the complex simulation models and the simple optimization models which are both found in the integrated assessment modelling community. I hope that such an approach may help to find insights in the complex problem of global change and yield possible long-term policy strategies. 


\section{7}

\section{The Initial Allocation of Emission Rights ${ }^{1}$}

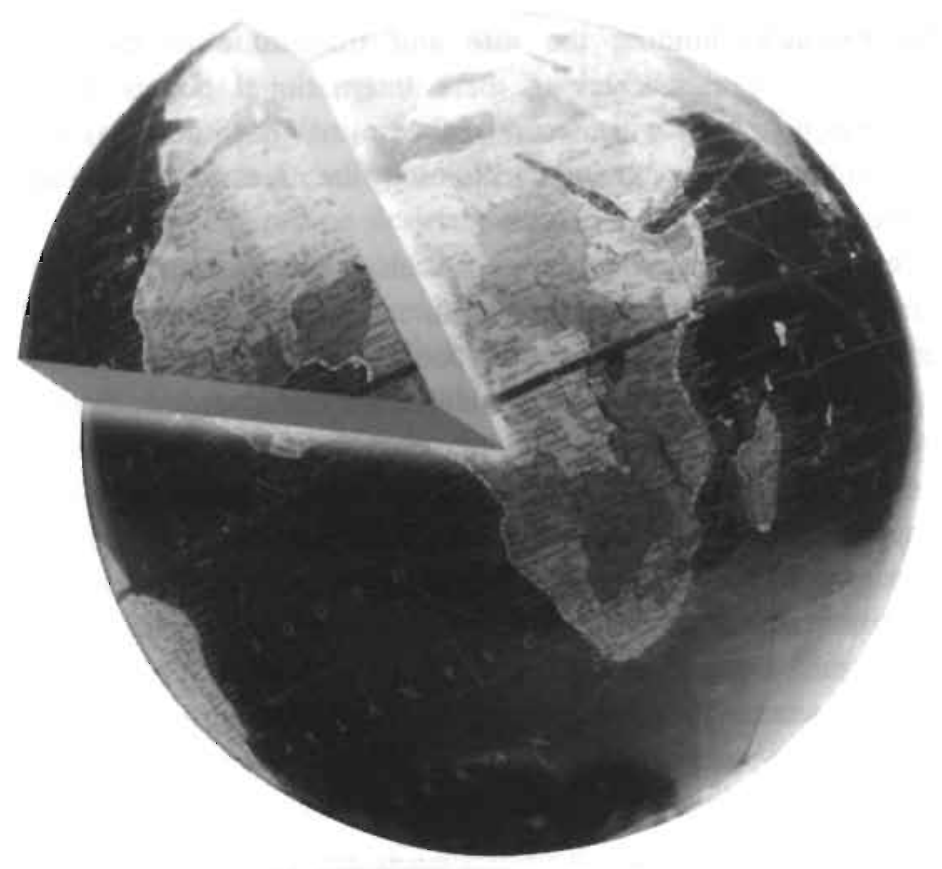

This Chapter is based on Janssen (1992), Den Elzen et al. (1992), Janssen et al. (1992) and Janssen. and Rotmans (1995). 


\subsection{Introduction}

The possibility that increasing atmospheric concentrations of radiatively active gases may lead to significant changes to global climate confronts society with a problem of unusual complexity. International response to reduce the expected risks of climate change is now considered appropriate by the majority of developed and developing countries. How strong a response is implemented depends on the evaluation of the risks of ecological and economic damage and costs of reducing emissions. As a tool for developing policies which limit the risks and effects of climate change, the Advisory Group of Greenhouse Gases (1990) identified several targets for climate change response strategies to protect the structure and functions of vulnerable ecosystems: for example, limiting the rate and magnitude of the change of temperature or of sea level. Achieving these international targets requires the implementation of policies that will involve reduction of greenhouse gas emissions.

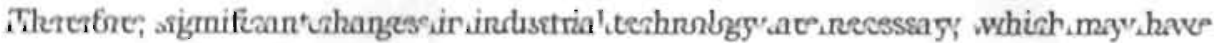
profound economic impacts on modern societies.

This brings us to another important issue of such an international climate policy: how will the responsibility for future reductions in greenhouse gases be allocated among countries? Regardless of ethical and political considerations, allocation of responsibility will have to take into account differences in economic development, technical know-how and demographic development, which largely characterize the different parts of the world both in the present state, as well as changes in history. In the early development stages, agriculture dominated the economy and emissions have been largely due to deforestation. In the next stages of industrialization and rapid growth of per capita income levels, emissions from the combustion of fossil fuels rapidly surpassed these biotic emissions.

This Chapter focuses on the greenhouse gas $\mathrm{CO}_{2}$ which is the most important anthropogenic greenhouse gas which contributes to an enhanced greenhouse effect. Furthermore, I mainly focus on emissions caused by fossil fuel combustion because of its relatively small uncertainties in measurements, its large share of total $\mathrm{CO}_{2}$ emissions (about 70\%) (IPCC, 1991), and because several other greenhouse gases are related to the combustion of fossil fuels $\left(\mathrm{CH}_{4}, \mathrm{CO}, \mathrm{NO}_{x}\right)$. In this way, I reduce the question of the responsibility of reducing greenhouse gas emissions to the question of the responsibility of reducing fossil $\mathrm{CO}_{2}$ emissions. In the analysis of the historical regional contributions of $\mathrm{CO}_{2}$ emissions, however, I will also indicate the contribution of $\mathrm{CO}_{2}$ emissions due to land use changes.

An important factor supporting the $\mathrm{CO}_{2}$ debate is the uniform mixing property of $\mathrm{CO}_{2}$ in the atmosphere. A ton of carbon emitted in Europe, for example, influences 
the climate in the same manner as the same ton of carbon emitted in Africa, for example. Recent findings on the local radiative forcing effects of sulphate aerosols (see Chapter 2), however, disturb the uniform mixing argument of trace gases. In the utopian case of sharing the responsibility of greenhouse gases, local climate change impacts, acidification impacts of $\mathrm{SO}_{2}$ emissions and carbon storage of the regional ecosystems should be taken into account. However, in developing a practical and simple tool to support decision-making and given the large uncertainties of local impacts, I hold on to the $\mathrm{CO}_{2}$ allocation issue.

A tool designed to stimulate cooperation and to facilitate the implementation of cost efficient measures is the concept of tradeable emission permits. Given an initial allocation of emission rights, actors will buy an amount of emission rights, if the costs of buying rights is less than investments to reduce the same amount of emissions in their own country.

One of the main problems in developing an effective international market for emission rights is the initial allocation of greenhouse gas emissions with respect to a constraint free anticipated or desired future. In theory, the optimal allocation of emission reductions depends on the marginal cost levels of the regions which cooperate and will not be influenced by an initial allocation of emission rights. However, the initial allocation will determine the flows of emission rights and, thus, also the assets between regions. Acceptance of an initial emission rights allocation will, therefore, largely depend on the resulting flows of emission permits.

Especially striking is the large "North-South" disparity in energy-related carbon emissions. Current per capita carbon emissions differ by nearly a factor of 9 . The burden of the developing countries is two-fold. The developing countries need to increase their per capita energy consumption in order to improve the quality of life and are also more vulnerable to adverse consequences of climate change. While industrialized countries are in a better position to achieve emissions reductions, they are also in a better situation to respond and adapt to climate change.

I begin this Chapter by estimating the historical regional carbon dioxide emissions due to fossil fuel combustion and land use changes and the regional contributions to the past rise in atmospheric $\mathrm{CO}_{2}$-concentration.

An egalitarian way to quantify the historical inequalities between world regions is introduced through the concept of "emission debt", based on an equal emission quotum per capita per year, irrespective of both the country a person lives in and the generation to which that person belongs. The emission debt is the amount of $\mathrm{CO}_{2}$ which some world regions have emitted over and above what they were allowed to, using an equal emission quotum per capita. The budgets left over can be considered as an egalitarian allocation of regional emission rights. 
Finally, an extension of the emission right allocation model is presented which involves the weighting of various criteria (e.g. size of population, gross national product [GNP], and energy consumption). A perspective-based uncertainty analysis is introduced to evaluate the influence of perspectives on the allocation of emission rights. On one hand, differences in perspectives lead to differences in preferred future developments, while on the other hand, the method for weighting indicators depends, among other things, on those perspectives.

\subsection{Historical Regional $\mathrm{CO}_{2}$-Emissions}

\subsubsection{Introduction}

In this Section, I will examine the regional $\mathrm{CO}_{2}$-emissions over the time period 1800 - 1990 from fossil fuel combustion (plus minor industrial sources like cement production) and changes in land use, which have been the primary cause of the observed increase in atmospheric $\mathrm{CO}_{2}$. I distinguish the following eleven regions:

- OECD: European Community (EC), Rest of Western Europe (RW.Eur.), OECD East (OECD E.), North America (N.Am.)

- Former centrally planned countries: Eastern Europe (E.Eur.), CIS (former Soviet Union)

- Centrally planned Asia (CPA)

- Other developing regions: Latin America (Lat.Am.), Africa, Middle East (M.East), and South/Southeast Asia (SSEA).

I will first discuss the $\mathrm{CO}_{2}$-emissions caused by fossil fuei combustion, followed by those caused by changes in land use.

\subsubsection{Fossil Fuel Combustion}

The global annual emissions of $\mathrm{CO}_{2}$ from fossil fuel burning and cement manufacturing (although the latter contributes less than $2 \%$ ) have shown an exponential increase since 1800 (about $3 \%$ yearly). There have been major interruptions during the two World Wars, the economic crisis in the 1930's and the oil crisis in the 1970's (see Figure 7.1). The cumulative release of $\mathrm{CO}_{2}$ from fossil fuel use from 1850 to 1987 is estimated at $201 \mathrm{GtC}$, which is within the uncertainty range of $200 \mathrm{GtC} \pm 10 \%$ (Marland et al., 1989). In 1989, the global emission was about 6.0 GtC (Marland et al., 1989). There is a main difference, however, between the contribution of the industrialized countries and the developing countries: about $85 \%$ 166 
of the fossil $\mathrm{CO}_{2}$-emissions in the past has been emitted by industrialized countries, where annual releases reach up to $5 \mathrm{tC}$ per capita (Rotty and Marland, 1986). In most developing countries, $\mathrm{CO}_{2}$-emissions are between 0.3 and $0.6 \mathrm{tC}$ per capita per year, although the relative rate of increase in the developing countries has been much larger during the last few decades, (about $5 \%$ per year in developing regions in contrast to $1 \%$ per year in the industrialized regions during the last decade). The historical $\mathrm{CO}_{2}$. emissions by fossil fuel combustion before 1800 were expected to be small, while the fossil fuel consumption in 1800 was already very low (Mitchell, 1981) and, thus, has a negligible contribution to the observed present atmospheric $\mathrm{CO}_{2}$ increase.

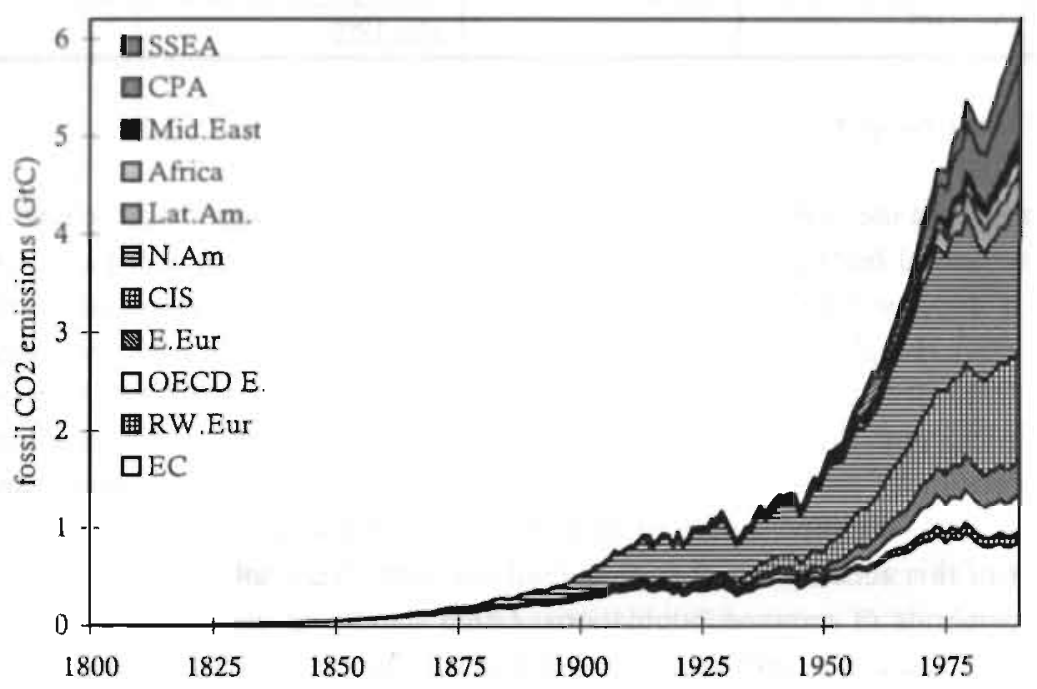

Figure 7.1: Regional $\mathrm{CO}_{2}$ emissions due to fossil fuel combustion and cement production over the period 1800 till 1990.

In Table 7.1, the sources are summarized which are used to estimate the fossil $\mathrm{CO}_{2}$ fluxes for the period 1800 - 1990 (see also Janssen, 1992). The differences between. total $\mathrm{CO}_{2}$-emissions calculated from the different sources and global emission data from Watts (1982), Marland and Rotty (1984), Rotty (1987), and Marland et al. (1989) are within the uncertainty ranges of about $5 \%$. 
Table 7.1: Sources used in estimating fossil $\mathrm{CO}_{2}$ fluxes

\begin{tabular}{|l|l|l|l|}
\hline Source & Time period & Data & $\begin{array}{l}\text { Type of data } \\
\text { and period used }\end{array}$ \\
\hline $\begin{array}{l}\text { Mitchell (1981" } \\
1982,1983)\end{array}$ & $1800-1975$ & $\begin{array}{l}\text { Production and } \\
\text { in- and export }\end{array}$ & $\begin{array}{l}\text { Only almost complete source of } \\
\text { national data for Europe, Russia, } \\
\text { North / Latin America } \\
\text { before 1925 }\end{array}$ \\
\hline $\begin{array}{l}\text { Darmstadter } \\
(1971)\end{array}$ & $1925-1965$ & Consumption & $\begin{array}{l}\text { National data for the whole world } \\
\text { 1925-1950 }\end{array}$ \\
\hline $\begin{array}{l}\text { Marland et al. } \\
(1989)\end{array}$ & $1950-1989$ & CO $_{2}$-emissions & $\begin{array}{l}\text { National data for the whole world } \\
\text { after 1950 }\end{array}$ \\
\hline
\end{tabular}

\subsubsection{Land Use Changes}

Changes in land use over the past two centuries have caused a significant release of $\mathrm{CO}_{2}$ from terrestrial biota and soils to the atmosphere. About one-third of past $\mathrm{CO}_{2}$ emissions is supposed to have come from land use changes (Siegenthaler and Oeschger, 1987; Houghton and Skole, 1990). During the 19th century, the emissions induced by land use changes were even larger than those from fossil fuels. Europe, North America, and the CIS have caused the largest contributions to emissions through expansion of croplands. In this century, land use changes in temperate and boreal zones have declined, while in tropical regions, they have accelerated. The major cause of this accelerating change in land use is the tremendous pressure from increasing demands of growing populations. Other damaging effects of large-scale changes in land use are the extinction of species, increased erosion, threats to indigenous people, and the destruction of a wide variety of possible important assets.

Estimates of $\mathrm{CO}_{2}$-emissions from land use changes depend on the rates of these changes, the amount of carbon in soil and biomass, rates of oxidation of wood products (through burning or decay) and rates of decay of organic matter in soil. In this study, the net release of regional $\mathrm{CO}_{2}$-emissions due to land use changes has been estimated for the period between 1800 and 1990 (see also Janssen, 1992). The changes in carbon storage are mainly caused by forest clearing, which converts forest to permanent agriculture and pasture. The contributions of selective logging and shifting cultivation are much smaller (Detwiler and Hall, 1988). Rates of land use changes for the period $1800-1980$ are derived from Houghton et al. (1983). The conversion rates are extrapolated for the period between 1980 and 1990. For other than tropical regions, the extrapolated rates are assumed to be the same as in 1980. For the tropical regions, the extrapolated. rates of conversion of ecosystems are based on FAO data 
(FAO, 1988; 1991). As Houghton et al. (1983) I have used a simple bookkeeping model, to calculate the yearly net changes of carbon in ecosystems (Figure 7.2), by using estimates of carbon in soil and vegetation before and after changes in land use (Houghton et al., 1983; 1987).

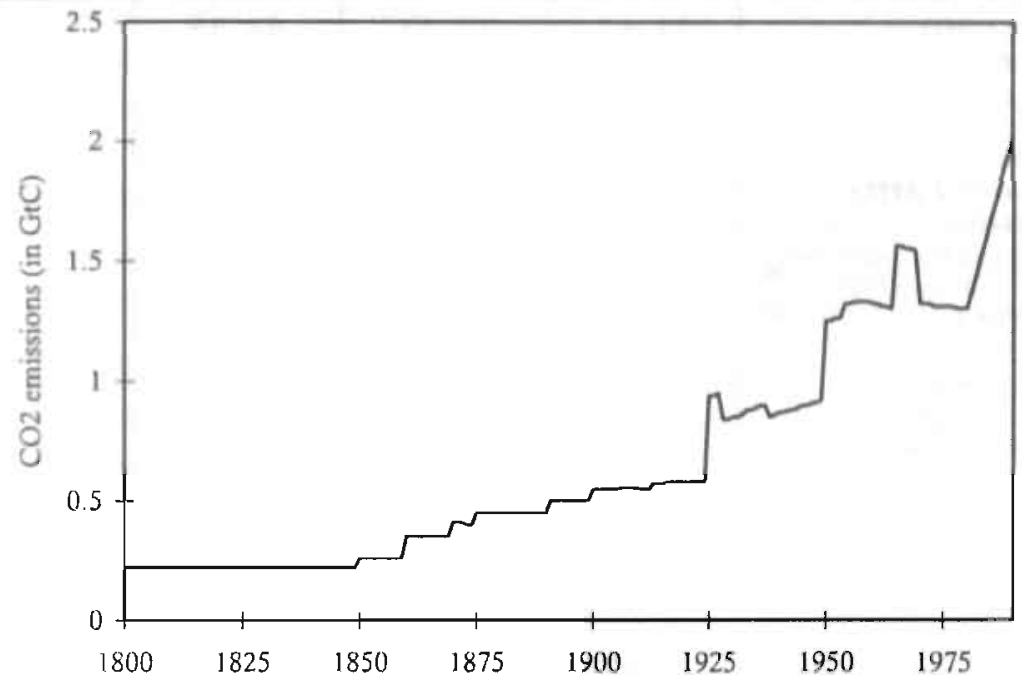

Figure 7.2: Global $\mathrm{CO}_{2}$ emissions due to land use changes.

Houghton (1991) gives four factors which cause uncertainties in estimating $\mathrm{CO}_{2}$ fluxes from land use changes. First, the rates of deforestation differ, depending on the study, even if they have used satellite imagery. This is largely because of differences in purpose and definitions. Secondly, large differences in estimates of carbon stocks cause uncertainties. Estimates vary by almost $100 \%$, which may be caused by possible errors in emission factors and differences in surveys (Houghton, 1991). Thirdly, differences are caused by the fate of deforested land or land use. Is deforestation permanent or temporary? Finally, uncertainties are caused by exchanges of biotic $\mathrm{CO}_{2}$ which are not associated with deforestation.

Comparisons of different studies show the large uncertainties in estimating $\mathrm{CO}_{2}$ emissions by land use changes (Table 7.2). The fluxes derived in this study for 1980 are within the uncertainty ranges of other studies, which is also the case in 1990 with fluxes in tropical regions. IPCC (1990) gives an $1850-1986$ estimate of $117 \pm 35 \mathrm{GtC}$, while the estimate over this period amounts to $107 \mathrm{GtC}$. 
Table 7.2 Ranges of $\mathrm{CO}_{7}$-emissions (in $\mathrm{GtC}$ ) from land use changes according to different studies.

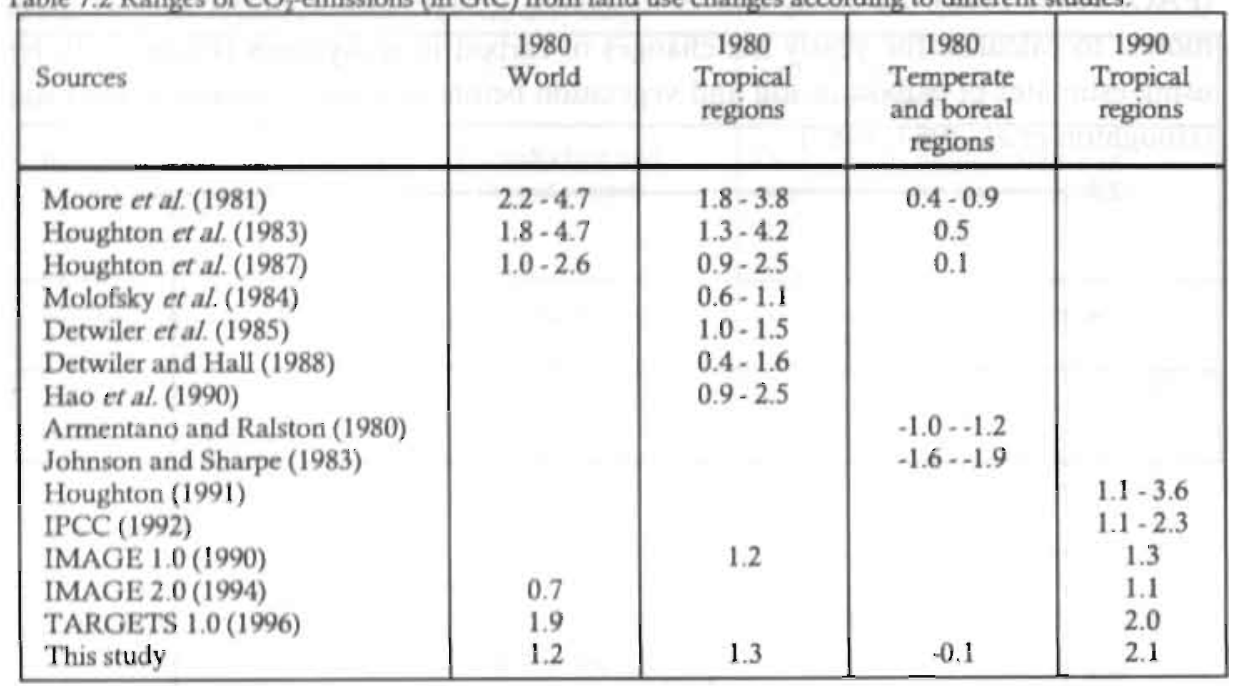

\subsection{Emission Debts}

\subsubsection{Historical Contribution to $\mathrm{CO}_{2}$ Concentration Rise}

Until now, industrialized countries have contributed much more to $\mathrm{CO}_{2}$-emissions than the developing world. Using the IMAGE 1.0 model, I have estimated the regional contribution both from fossil fuel use and land use changes, to the increase in the $\mathrm{CO}_{2}$-concentration since 1800 . This has been done by calculating the difference in $\mathrm{CO}_{2}$-concentration increase in 1990 with and without the emissions of the region under concern. For these calculations, IMAGE 1.0 had to be adapted in order to start: in 1800. For the ocean model, the initial values of the amount of carbon in the surface layers were taken from Goudriaan and Ketner (1984). The deforestation and terrestrial modules are set off in this version for practical reasons. This implies that the $\mathrm{CO}_{2}$-uptake by the teriestrial biosphere through negative feedbacks is not simulated, resulting in an unbalanced carbon budget over the period in the past, and a simulated $\mathrm{CO}_{2}$-concentration of $371 \mathrm{ppmv}$ in 1990 (while the observed value is $354 \mathrm{ppmv}$ ). Also the fact that the $\mathrm{CO}_{2}$-fluxes from land use changes in this study are higher than those of IMAGE 1.0 causes a higher concentration level, but this does not affect the relative contributions of the regions.

Figure 7.3 shows the relative contributions of the world regions to the rise in atmospheric $\mathrm{CO}_{2}$-concentration. The contribution from Western. Europe and North 
America amounts to about $40 \%$; for Eastern Europe (including the CIS) about $20 \%$, and $\mathrm{OECD}$ East about $5 \%$, the combustion of fossil fuels being the major cause. On the other hand, the relative contributions from Africa, Latin America and South/Southeast Asia (exceeding 30\%) are for about $75 \%$ due to land use changes.

When the relative part of emissions in the past is used to estimate the regional contribution (e.g. Krause et al., 1989) to climate change, regions with relatively large emissions in the last century will be overestimated, in contrast to those of which emissions have accelerated in the last few decades. This overestimation is caused by the atmospheric lifetime of $\mathrm{CO}_{2}$, which is about 50 to 200 years (IPCC, 1990).

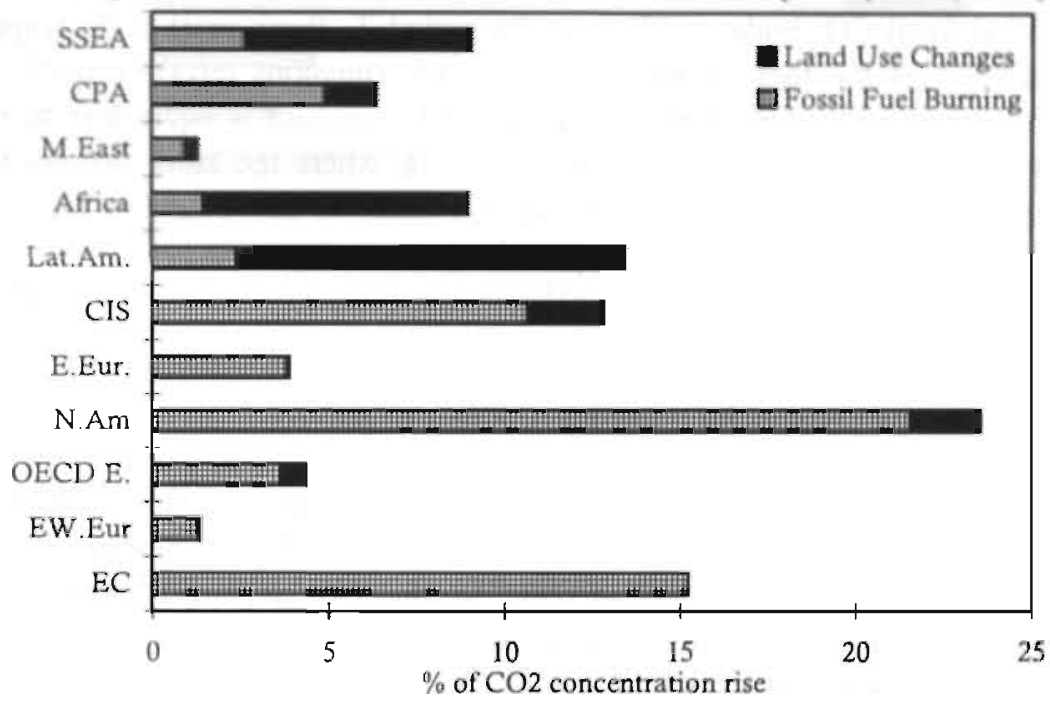

Figure 7.3: Relative regional contributions to the $\mathrm{CO}_{2}$ concentration rise caused by fossil fuel combustion and land use changes.

\subsubsection{The Concept}

The natural carbon cycle involves exchanges of carbon dioxide between the atmosphere, the oceans, and the terrestrial biosphere which amount to hundreds of billions of tons of carbon per year. The extra anthropogenic emissions associated with. land-use changes and fossil fuel combustion appear to be relatively small in comparison with such enormous quantities, but are, nevertheless, believed to account for an imbalance in the carbon cycle, which has caused an increase in the atmospheric $\mathrm{CO}_{2}$ concentration during the past century. Because oceans and terrestrial ecosystems. 
act as sinks for large amount of $\mathrm{CO}_{2}$, only about $40 \%$ of the anthropogenic emissions remain in the atmosphere.

If international policy targets based on maximum $\mathrm{CO}_{2}$ concentrations are to be met, anthropogenic emission levels will have to remain below a target level during a specified period. Such policy targets, thus, imply that only a limited amount of anthropogenic $\mathrm{CO}_{2}$ emission may be permitted during a fixed period, an amount referred to as "the global $\mathrm{CO}_{2}$ emission budget". This budget takes account of historical, as well as projected future contributions.

The global fossil $\mathrm{CO}_{2}$ budget (GB), over the period $T_{\mathrm{B}}$ (base year) to $\mathrm{T}_{\mathrm{E}}$ (expiry year) is defined as the total of regional fossil $\mathrm{CO}_{2}$ emissions $(e(t, r))$ emitted, or considered to be permissible during that period. This amount is equivalent to the cumulative historical $\mathrm{CO}_{2}$ emissions (from $T_{n}$ to $T_{R}$. where the latter denotes the reference year) and future, scenario-dependent $\mathrm{CO}_{2}$ emissions (from $\mathrm{T}_{\mathrm{R}}+1$ to $\mathrm{T}_{\mathrm{E}}$ ):

$$
G B=\sum_{s}\left[\sum_{t=T_{*}}^{T_{A}} e_{\text {hix }}(t, r)+\sum_{t=T_{n+1}}^{T_{A}} e_{\text {secen }}(t, r)\right]
$$

where $\mathrm{r}=$ region and $\mathrm{t}=$ time; his $=$ historical, scen $=$ scenario.

If the world community is to accept constraints on $\mathrm{CO}_{2}$-emissions, how can future $\mathrm{CO}_{2}$ - emission rights be allocated to the world regions? In the last Section, it was shown that the $\mathrm{CO}_{2}$-concentration increase in the past is largely caused by industrialized regions, while Figure 7.4 shows that historical $\mathrm{CO}_{2}$-emissions per capita are unevenly divided among the world regions. In this Section, I will try to quantify the fact that some regions have emitted more $\mathrm{CO}_{2}$ in the past than they were allowed to by equity rules. This so-called emission debt is defined here in terms of population levels. 


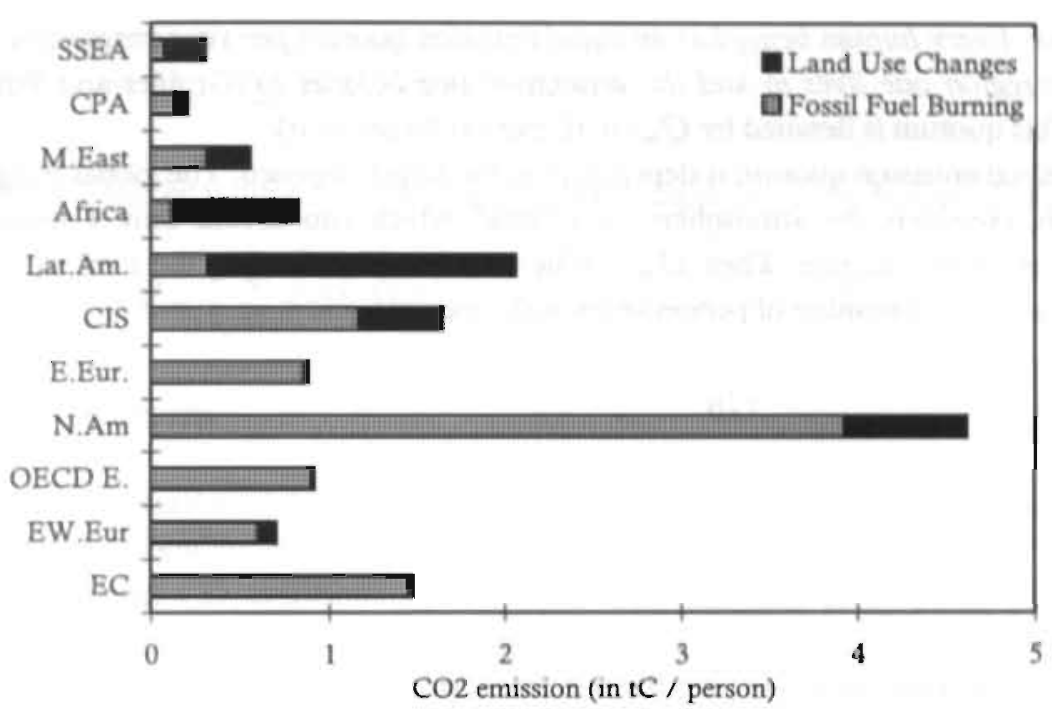

Figure 7.4: Average emission per capita for the period between 1800 and 1990 . Regional differences exist in sources and levels of emissions.

Although the results in this Section are based on population levels, several other indicators for allocating future emissions to regions can be considered. Actually, the process of finding acceptable indicators as a base for allocation of payments or permits is now gaining momentum, as part of the attempts to set up a global climate agreement. Indicators for such an allocation could be based on present and past emissions, area, population levels (equal allocation per capita carbon budget), state of the economy (equal allocation per unit GNP budget), energy intensity or a combination of these factors (Krause et al., 1989; Grubb, 1989; Grübler and Fujii, 1991; Grubb and Sebenius, 1992).

Because the per capita quotum approach ${ }^{z}$ is conceptually attractive and computationally simple, it is used here for quantifying the egalitarian thesis that some regions have emitted more in the past than they were allowed to. The per capita approach can be translated into the following equity ideal for the allocation of the

2 The population data between 1800 and 1920 are based on Durand (1967) and the population data covering $1920-1990$ have been obtained from the United Nations World Population Prospects (UN, 1966; UN, 1990a). The future population figures are based on IPCC (1992). Global population figures are estimated to increase from 5.3 billion in 1990 to 11.3 billion in 2100 , mainly due to the growth in the developing countries. 
emissions: Every human being has an equal emission quotum per year irrespective of both the region one lives in and the generation one belongs to (Grübler and Fujii, 1991). This quotum is denoted by $Q_{\text {aup }}$ (in tC per capita per year).

The equal emission quotum is dependent on the target imposed. The global budget approach, considers the atmosphere as a "sink" which can absorb only a limited amount of carbon dioxide. Then $Q_{\text {rap }}$ can be calculated by dividing the total carbon budget by the total number of person years in this period:

$$
Q_{\text {cap }}=\frac{G B}{\sum_{r}\left(\sum_{t=T_{n}}^{T_{1}} \operatorname{pop}_{\text {hi }}(t, r)+\sum_{t=T_{n+1}+1}^{T_{E}} \operatorname{pop}_{\operatorname{sen}}(t, r)\right)}
$$

where pophis $(t, r)$ and pop $p_{s c e n}(t, r)$ are the historical and future regional population size, respectively, in year $t$ for region $r$.

I will now define the regional emission debt as the difference between the amount of $\mathrm{CO}_{2}$ allowed to be emitted by the regional population [pop(t,r) in $10^{9}$ persons], based on the equal quotum per capita $\left[Q_{\text {rap }}\right.$ in tC per (cap x year)] and the actually cumulated regional emissions in the past $[e m(t, r)$ in $\mathrm{GtC}$ per year]. The emission debt in $T_{R}[E D(r)$ in $\mathrm{GtC}]$ is described in mathematical terms:

$$
E D(r)=\sum_{r=T_{s}}^{T_{A}} e m(t, r) \cdot Q_{\text {cap }} \cdot \sum_{t=T_{\theta}}^{T_{A}} p o p(t, r)
$$

Note that 1 have assumed that the emission data is gained by measurements of regional emissions and not by regional end-use of production. Ignoring the transactions between regions may bias the results. However, for lack of sufficiently reliable data, especially those related with land use changes, I am forced to use the regional emissions.

How should the remaining global carbon budget be distributed over the different regions, taking into account past and present emissions of $\mathrm{CO}_{2}$ ? Here I focus on a simple allocation rule for the remaining carbon budget based on population size. The future $\mathrm{CO}_{2}$-emission rights [ER(r)] consist of the $\mathrm{CO}_{2}$-emissions allowed to be emitted in the period $T_{R}+1$ to $T_{\mathrm{E}}$, based on the equal emission quotum minus the emission debt built up in the past: 


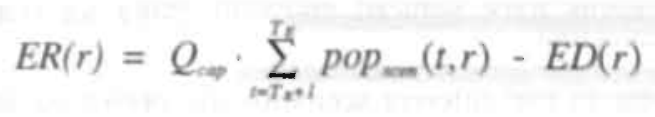

This can also be written as the regional carbon budget minus the historical emissions:

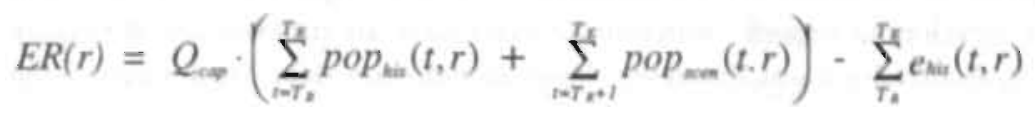

\subsubsection{The Results}

Three scenarios of the IPCC (1992) (see also Appendix) are used to define a range of global carbon budgets. Remaining budgets for the scenarios are listed in Table 7.3 for both total $\mathrm{CO}_{2}$-emissions and for fossil $\mathrm{CO}_{2}$-emissions only. These budgets are between $710 \mathrm{GtC}$ and $2226 \mathrm{GtC}$. Krause et al. (1989) derived a global remaining budget of about $300 \mathrm{GtC}$ for fossil $\mathrm{CO}_{2}$-emissions based on a $400 \mathrm{ppmv} \mathrm{CO}_{2}$ concentration target, including fossil fuel $\mathrm{CO}_{2}$-emissions between 1985 and 2100 .

The budgets are significantly higher than various studies propose as sustainable futures (e.g. Krause et al., 1989; Den Elzen et al., 1992), although IS92C leads to a stabilization of projected temperature change of $2^{\circ} \mathrm{C}$ above 1990 (Den Elzen, 1993).

\begin{tabular}{|c|c|c|c|}
\hline Scenario & $\begin{array}{l}\text { Remaining } \\
\text { budget (GtC) } \\
\text { Tot." Ind. }\end{array}$ & $\begin{array}{l}Q_{\text {asp }} \\
\text { [tC per (cap x year)] } \\
\text { Tot. }\end{array}$ & $\begin{array}{l}Q_{\text {owp }} \\
\text { (tC per (cap x year)] } \\
\text { Ind. }\end{array}$ \\
\hline IS $92 \mathrm{~A}$ & $1543 \quad 1458$ & 1.36 & 1.21 \\
\hline IS $92 \mathrm{C}$ & 710 & 0.97 & 0.80 \\
\hline IS $92 \mathrm{E}$ & $2226 \quad 2141$ & 1.86 & 1.70 \\
\hline
\end{tabular}

Table 7.3: Carbon budgets for the used IPCC 92 scenarios and $Q_{\operatorname{oug}}$ values [in $\mathrm{tC}$ per(cap $\mathrm{x}$ year)].

"Tot. : $\mathrm{CO}_{2}$-emissions from fossil fuel use and land use changes.

${ }^{b}$ Ind. : $\mathrm{CO}_{2}$-emissions from fossil fuel use.

Based on this per capita quotum, the global carbon budget for the period 1800 to 2100 under the IS92C scenario of about $1130 \mathrm{GtC}$ corresponds with a permitted emission per capita of $0.97 \mathrm{tC}$. Under the high emission IS92 E scenario, Q amounts to $1.86 \mathrm{tC}$.

There is no global emission debt from past to future generations using the per capita quotum of the IPCC scenarios. In Den Elzen et al. (1992), smaller carbon budgets are used, based on more severe climate targets, so that a global emission debt 
would exist so that we and our ancestors have reduced emission quota for our descendants.

The resulting regional emission debts in the different scenarios are presented in Figure 7.5. The industrialized regions are, in most cases, highly indebted, in contrast to. Asian regions. Excluding land use changes leads to a large decline of emission debts in most developing regions, which in all cases have an emission credit (Figure 7.6). The industrialized regions, however, have an emission debt for almost all the budgets.

After having estimated the regional emission debts, the emission rights left for the different world regions can now be calculated. Figure 7.7 summarizes the emission rights per capita per year based on different carbon budgets. Almost all industrialized regions and Latin America have emission rights per capita, which are all lower than present emission levels. North America has emitted more $\mathrm{CO}_{2}$ in the past 191 years than was allowed for the whole period of 301 years and ends up with negative emission permitted for all scenarios. Most of the developing regions, however, have emission permitted per capita which are higher than the emission rights of industrialized regions, but in sorne cases lower than their present emission levels. This means that in a world striving towards sustainable development and equity, emission levels per capita are not allowed to increase, not even in developing regions.

When considering only fossil $\mathrm{CO}_{2}$-emissions, the emissions permitted per capita in developing regions are somewhat higher than for land use changes, but they will never reach the present emission levels of the industrialized regions. If developing regions are empowered and supported to continue their development towards higher standards of living, large technological improvements will have to be realized. 


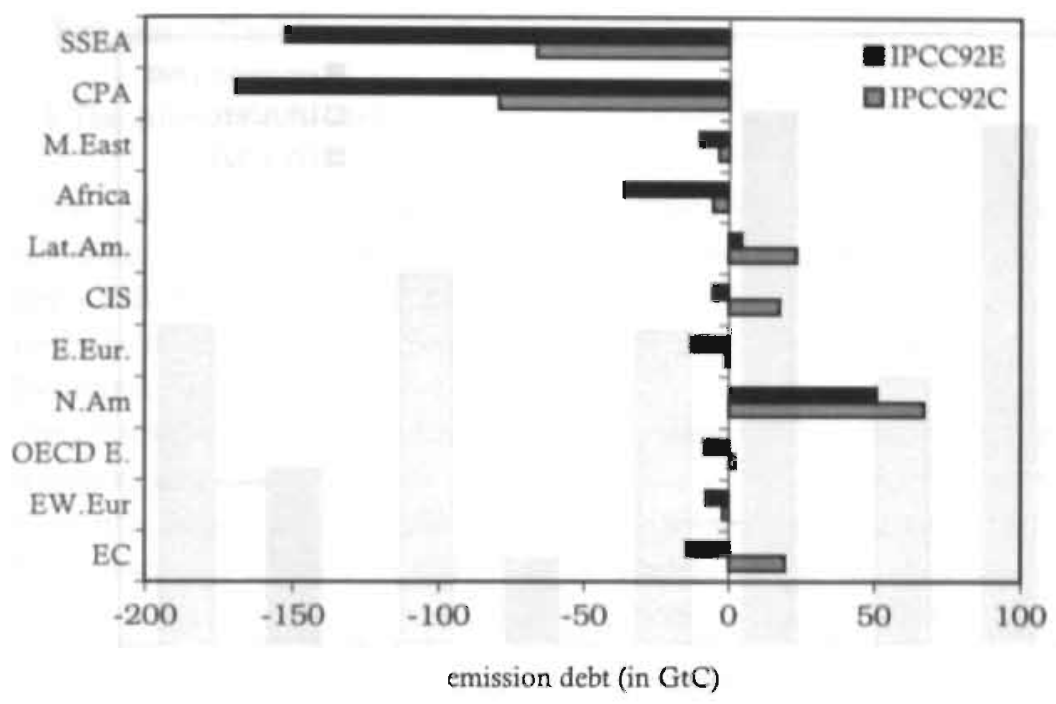

Figure 7.5: Regional emission debts for fossil fuel combustion and land use changes between 1800 and 1990, using different carbon budgets.

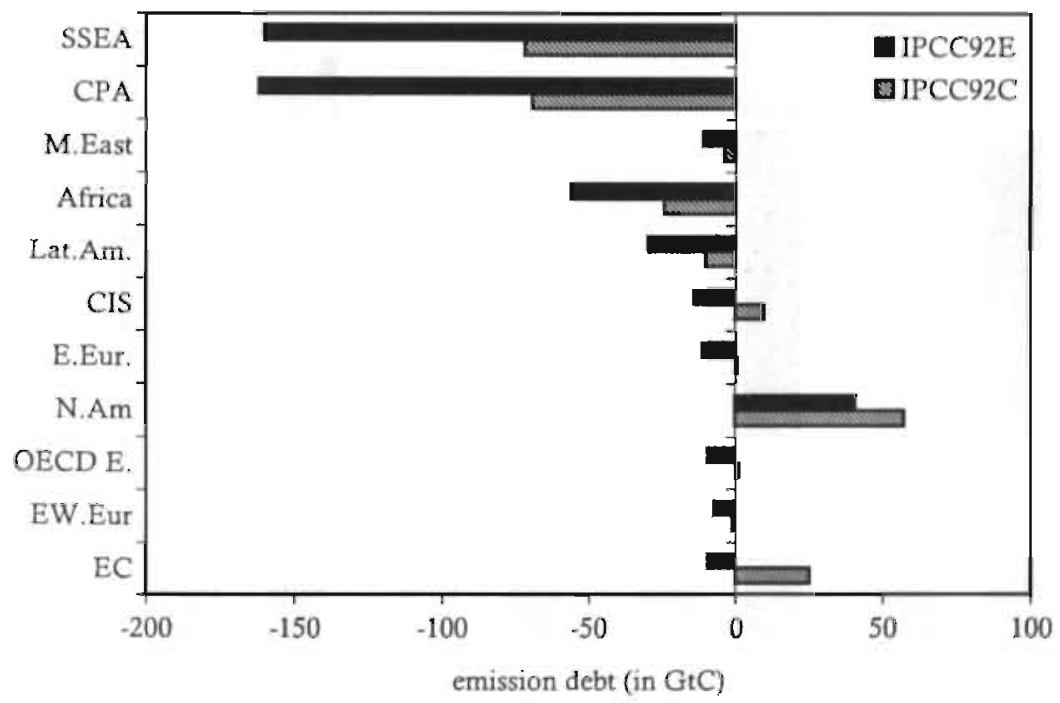

Figure 7.6: As Figure 7.5 without land use changes. 


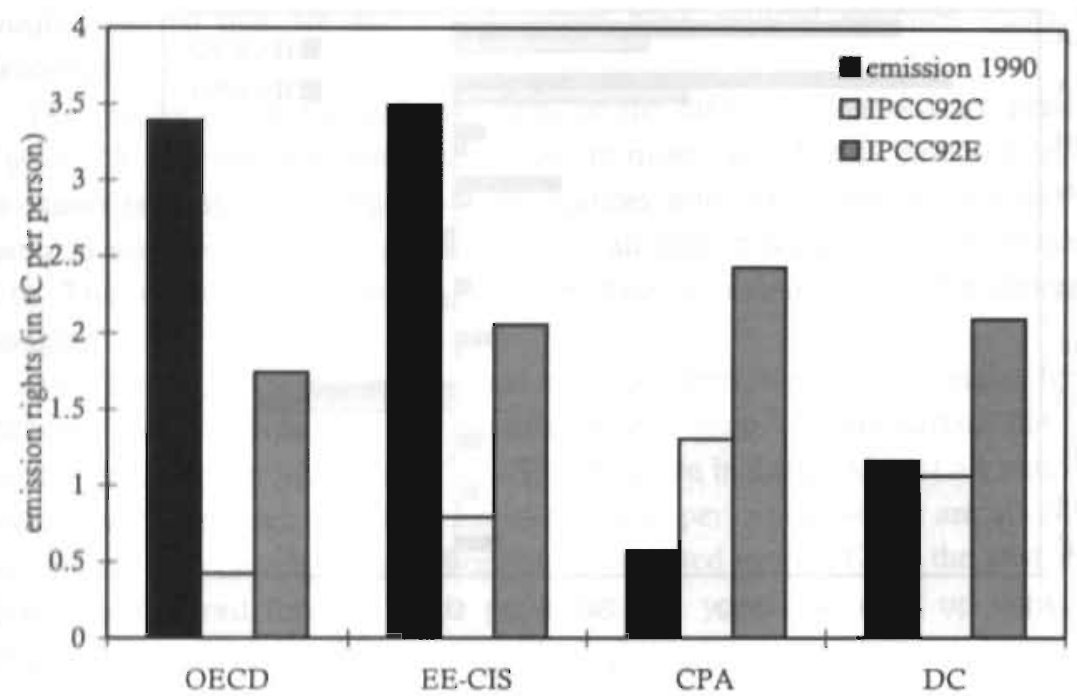

Figure 7.7: Regional emission rights per capita for fossil fuel combustion and land use changes for the period $1991-2100$, using different carbon budgets.

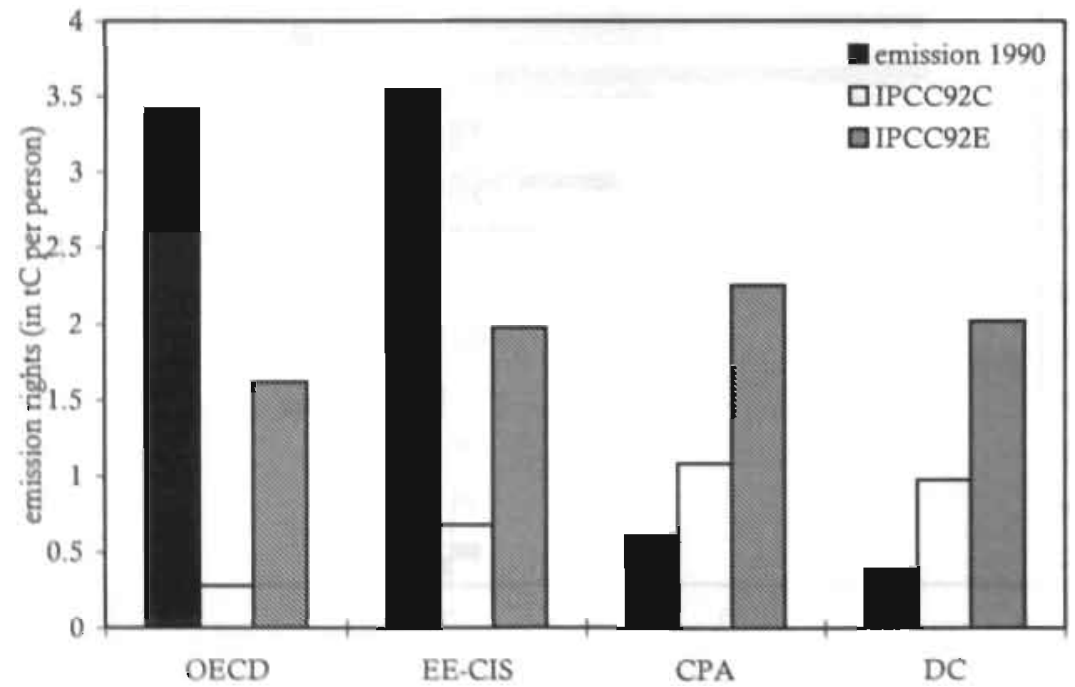

Figure 7.8: As Figure 7.7 without land use changes. 


\subsection{Emission Rights}

\subsubsection{The Allocation Model}

The distributions in the last Section are based on a rather egalitarian criteria: population size. A more sophisticated allocation model including various allocation criteria will be presented in this Section. Furthermore, I will introduce a perspectivebased analysis to quantify subjective judgments in order to scan the range of possible allocations.

Since fossil $\mathrm{CO}_{2}$ (i.e. $\mathrm{CO}_{2}$ from fossil fuel combustion) is the main contributor to anthropogenic $\mathrm{CO}_{2}$ emissions (about 70-90\%) I will consider only fossil $\mathrm{CO}_{2}$ emissions. Target scenarios adopted as policy by the world community imply that only a restricted amount of $\mathrm{CO}_{2}$ may be emitted in the future. I define regional emission rights (ERs) for the future as the fractions per region (FRs) of the global $\mathrm{CO}_{2}$ budget, i.e. permitted emissions in region $\mathrm{r}$ during the entire period under consideration minus the historical $\mathrm{CO}_{2}$ emissions:

$$
E R(r)=F R(r) \cdot G B-\sum_{t=T_{\sharp}}^{T_{R}} e_{h i s}(t, r)
$$

The regional fraction (FR) depends on a combination of various criteria. For simplicity's sake, I will first present the case in which fossil $\mathrm{CO}_{2}$ emission rights are allocated according to the single criterion of population size. Thereafter, the approach is extended to enable a weighted cluster of criteria to be incorporated in the model.

If every person living in the period between $T_{B}$ and $T_{E}$ is credited with equal annual fossil $\mathrm{CO}_{2}$ emissions, the annual regional fraction can be expressed as follows:

$$
\begin{aligned}
& f r(t, r)_{p o p}=\frac{p o p_{h i s}(t, r)}{\sum_{r}\left[\sum_{r=T_{t}}^{T_{k}} \operatorname{pop}_{h i s}(t, r)+\sum_{r=T_{k}+1}^{T_{k}} \operatorname{pop}_{s c e n}(t, r)\right]} \quad \text { for } t \leq T_{k} \\
& f r(t, r)_{p o p}=\frac{p o p_{\text {scen }}(t, r)}{\sum_{r}\left[\sum_{t=T_{R}}^{T_{R}} p o p_{h i s}(t, r)+\sum_{t=T_{R}+1}^{T_{\varepsilon}} p o p_{\text {seen }}(t, r)\right]} \quad \text { for } t>T_{R}
\end{aligned}
$$

where:

pop $_{\text {his }}=$ population during the historical period;

pop $_{\text {sen }}=$ scenario-dependent population projections for a future period. 
The total regional fraction $\mathrm{FR}_{\text {pop }}(\mathrm{r})$ is the sum of the annual regional fractions, $\mathrm{fr}_{\mathrm{pop}}(\mathrm{t}, \mathrm{r})$ :

$$
F R_{p o p}(r)=\sum_{t=T_{s}}^{T_{s}} f r_{p o p}(t, r)
$$

To incorporate time preference, the population size in the past and future will be discounted by introducing the discount parameter, $\delta$, where it goes without saying that discounting past responsibility differs from discounting future projections. However, for simplicity's sake, I assume that both past and future developments can be discounted by the same discount parameter $\delta$.

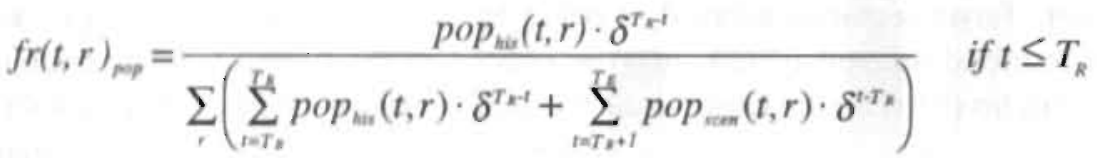

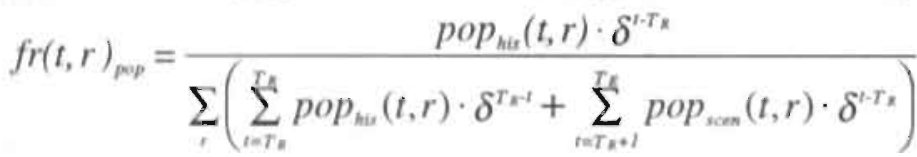

In order to incorporate a cluster of criteria rather than considering the "egalitarian" criterion of population size alone, the regional fraction will be extended to a weighted value of allocation indicators. Among such indicators are criteria such as: present and past emissions, population levels, state of the economy, energy use, and land area, or a combination of these (Krause et al., 1989; Grubb, 1989; Grübler and Fujii, 1991; Grubb and Sebenius, 1992; Rose, 1992). In this Section, the regions have been allocated a restricted fossil $\mathrm{CO}_{2}$ emission budget based on a weighted combination of the indicators: population size (per capita equity), GNP (economic affluence) and primary energy use (efficiency), because these are the main underlying factors associated with an increase in fossil $\mathrm{CO}_{2}$ emissions.

$$
F R(r)=\alpha_{1} \cdot F R_{\text {pep }}(r)+\alpha_{2} \cdot F R_{\text {gmp }}(r)+\alpha_{3} \cdot F R_{\text {enerog }}(r)
$$

where:

$\alpha_{1}=$ fractional weight of population

$\alpha_{2}=$ fractional weight of GNP

$\alpha_{3}=$ fractional weight of energy use

and where:

$\alpha_{1}+\alpha_{2}+\alpha_{3}=1$. 
Thus, the allocation of a $\mathrm{CO}_{2}$ emission budget for the period $T_{R}$ to $T_{R}$ to an important degree depends, in modelling terms, on the parameters; $\alpha_{1}, \alpha_{2}, \alpha_{3}, \delta$ and $T_{b}$. The preferred values of the parameters can vary according to the perspective(s) upon which subjective judgments are based.

\subsubsection{Emission Rights Allocation Based on Uniform Parameter Distributions}

In this Section, an approach is introduced which is designed to generate statistical distributions for regional fossil $\mathrm{CO}_{2}$ emission rights. For each parameter of the allocation model, an uncertainty interval can be found. The parameters $\alpha_{1}$ are assumed to vary between 0 and 1 . Because the sum of the set of $\alpha_{1}$ equals one, they form a simplex of possible $\alpha_{4}$ values. The interval for the discount parameter $\delta$ is assumed to be $[0.9,1.0]$. The base year $\left(\mathrm{T}_{\mathrm{B}}\right)$ varies between 1800 , which is taken as the beginning of the industrial revolution, and $\mathrm{T}_{\mathrm{k}}$.

It is to be expected that, having adopted different perspectives, actors will express dissimilar preferences for parameter values. In order to estimate the influence of these differing preferences, $I$ assume that they can be represented by a distribution plotted in terms of an uncertainty interval. Such distributions represent actors' preferences for parameter values, and are used because I assume that policy-maker's preferences are imprecise and distributed around a preferred value. This procedure enables the fuzziness of the preferences to be taken into account.

For the purposes of this Section, I assume that preferred values for $\delta$ and $T_{B}$ are uniformly distributed on the uncertainty interval. The $\alpha_{i}$ values are uniformly distributed on the simplex. If I may assume that all actor preferences are uniformly distributed, allocations which I subsequently derive can be used to estimate the range of preferred allocations.

The reference scenario (IS92A) published by the IPCC (1992) has been used to derive distributions of regional emission rights ${ }^{3}$. The allocated global emission budget

\footnotetext{
The scenarios for fossil $\mathrm{CO}_{2}$ emissions, population, GNP and primary energy use are based on the IPCC (1992) scenarios. In the IPCC. scenarios the following 4 world regions are: distinguished: OECD, Eastern Europe and the Community of Independent States (EE-CIS), Centrally Planned Asia (CPA) and the Developing Countries (DC). The main assumptions in. the IPCC scenarios are given in the appendix. The historical estimates for GNP levels are based on growth rates as given in Mitchell (1981, 1982, 1983), IMF (1992), UNCTAD (1985) and 1987 values of regional GNP levels as given in WRI $(1990 / 1991)$ and UN (1990b). Historical data for primary energy use are derived from Darmstadter (1971), Mitchell (1981, 1982. and 1983) and OECD (1989, 1991a, 1991b, 1991c, 1992).
} 
addresses emissions in the period $1991\left(T_{R}=1990\right)$ to $T_{E}$. An extensive range $e^{4}$ of regional emission rights was obtained by running the allocation model using different parameter values for each run. The parameter values are derived from a uniform distribution. The cumulative "probability" of regional emission rights for the period 1991 - 2000 is depicted in Figure 7.9. The emission rights accruing to the industrialized regions (OECD and Eastern Europe and the Community of Independent States [EE-CIS]) are, in about $90 \%$ of the cases, between - 30 and $30 \mathrm{GtC}$ and between -5 and $10 \mathrm{GtC}$, respectively. For Centrally Planned Asia (CPA), the $90 \%$ confidence interval lies between 5 and $20 \mathrm{GtC}$ and, in the case of the developing countries (DC), between 25 and $70 \mathrm{GtC}$. This implies that if perspectives are uniformly distributed, and scenario IS92A is adopted, the emission rights for the period 1991-2000 lie within the above bounds.

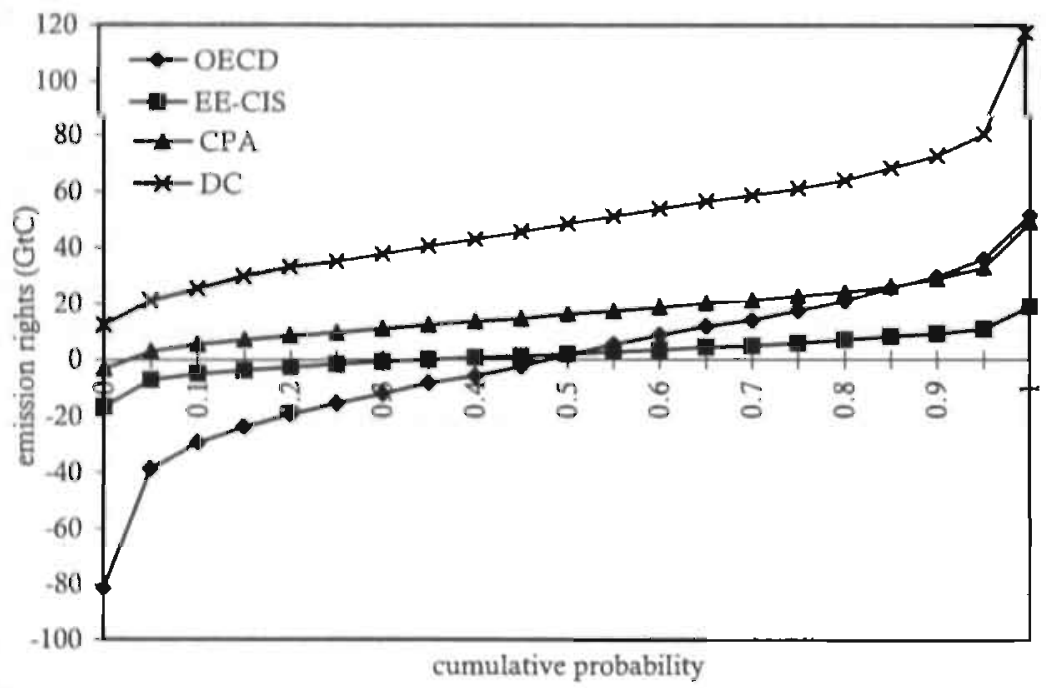

Figure 7.9: Cumulative probability of regional $\mathrm{CO}_{2}$ emission rights using a uniform distribution for the parameter values (1991-2100).

4 Since the distribution function of the regional emission rights, which is based on the distributions of the parameter values, is not known, I could not formally determine the minimum number of simulation runs. Experiments show that the mean and standard deviation converge after several hundred runs. Therefore the number of runs used in this study was set at 1000 
Figure 7.10 depicts the relative regional shares of the mean values of the global fossil $\mathrm{CO}_{2}$ emission budget for several values of the time horizon $\mathrm{T}_{\mathrm{E}}$. A remarkable result is that the relative distribution is stable after 2050, an effect mainly caused by the impact of historical emissions reducing the emission rights of industrialized regions, where short-term horizons are concerned.

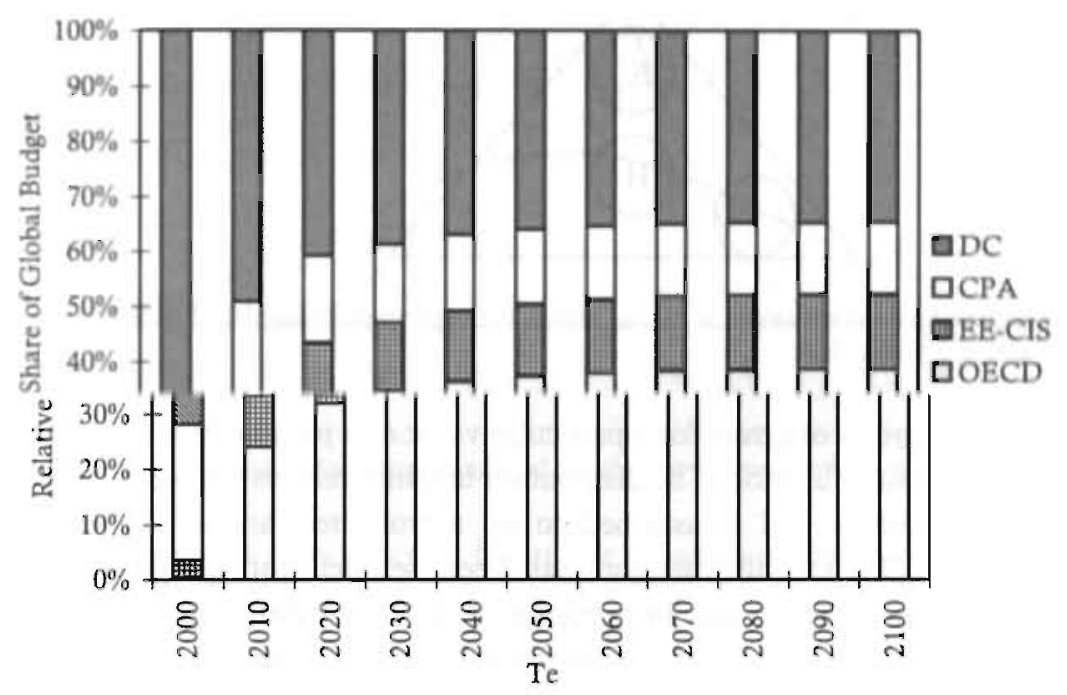

Figure 7.10: Relative regional share of the global $\mathrm{CO}_{2}$ emission budget for different values of $\mathrm{T}_{1}$

\subsubsection{Emission Rights and Perspectives}

\section{Perspective-based Parameter Distributions}

The aim in this Section is to quantify the influence of perspectives on the allocation of fossil $\mathrm{CO}_{2}$ emission rights; therefore, I need a distribution function for the preferences of parameter values.

As an introduction to the perspective-based parameter distribution functions, I will discuss the choices for the values of $\alpha_{1}, \alpha_{2}$ and $\alpha_{3}$. In Section 7.4.2, preferences of a group were assumed. to be uniformly distributed across the entire simplex. In this Section, however, I will assume that actors with different perspectives prefer certain parameter values to at certain degree of preference. For simplicity's sake, I assume that egalitarians base their allocation on population size (equity per capita), individualists on GNP (economic affluence), and hierarchists on a mixture of criteria (compromise). 
For the egalitarians (E), the preferred area of parameter values of $\alpha_{4}$, therefore, tends towards a high value of $\alpha_{1}$, vis-à-vis other perspectives (Figure 7.11).

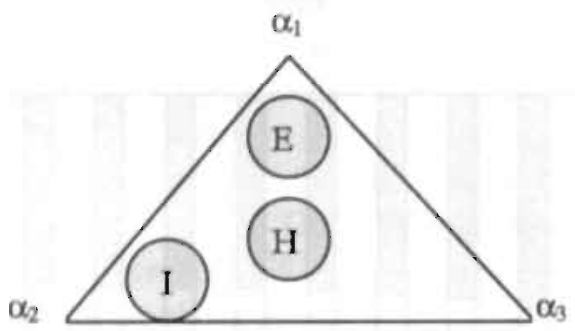

Figure 7. il: Preferred parameter values of $\alpha_{1}, \alpha_{2}$ and $\alpha_{3}$ in the perspective simplex. E=Egalitarian, I = Individualist and $\mathrm{H}=$ Hierarchist.

Preferences of a group of actors for a particular value of a parameter are described in terms of a distribution function. The distribution function reflects the preference for certain parameter values and is assumed to be a truncated normal distribution function (Equation 7.10). This function has been selected for its approximate symmetry and the fact that it can be represented by two parameters. Of course, selecting this particular distribution function is somewhat arbitrary and several other functions might have been used. The parameter value for which the density function reached a peak value $\left(\sigma_{1}\right)$ is called the preferred value. The variance can be influenced by the parameter $\sigma_{2}$, which can be interpreted as expressing the degree of preference. Here, a higher value of $\sigma_{2}$ reduces the variance of the preference and, therefore, represents a more pronounced preference (see Figure 7.12). 


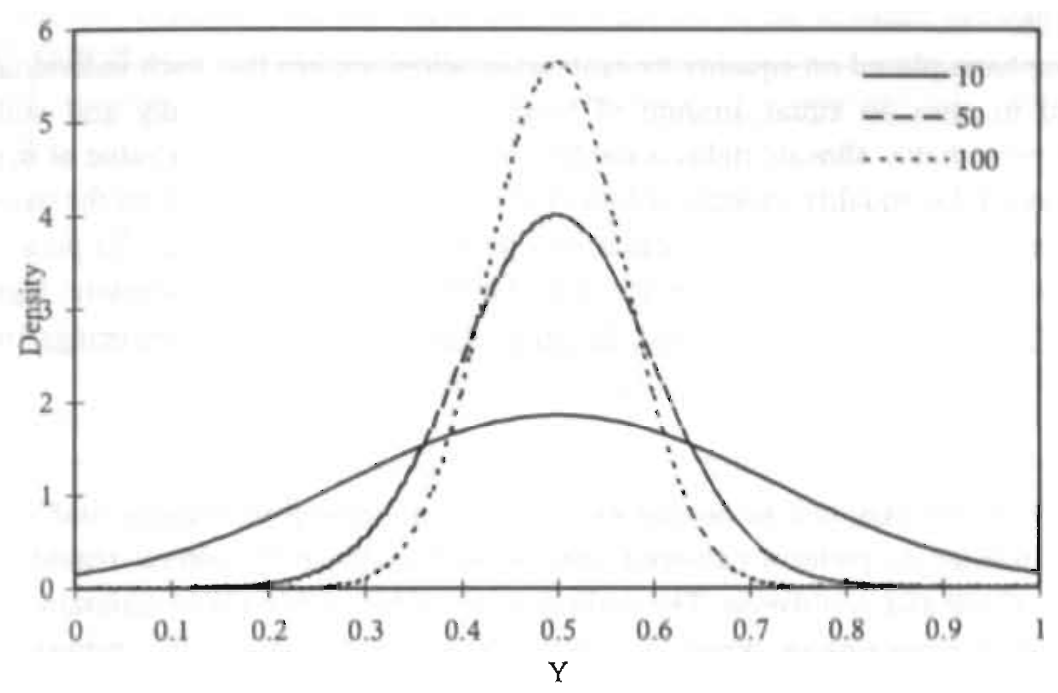

Figure 7.12: The density of a truncated normal distribution function with $\sigma_{1}=0.5$, for there values of $\sigma_{2}$.

The general density function $f(x)$ of the parameters in the uncertainty range $[a, b]$ can now be written as follows:

$$
\begin{aligned}
& f(x)=\frac{1}{c} \cdot e^{-\sigma_{z} \cdot\left(\frac{x-\sigma_{1}}{b-a}\right)^{2}} \quad \text { for } a \leq x \leq b \\
& \text { where: } \\
& c=\sqrt{\frac{\pi}{4 \sigma_{2}} \cdot\left(1-e^{-\sigma_{2} \cdot\left(\frac{\left.a \cdot \sigma_{t}\right)^{2}}{b-a}\right)}\right.}+\sqrt{\frac{\pi}{4 \sigma_{2}} \cdot\left(1-e^{-\sigma_{2} \cdot \frac{b \cdot \sigma_{t} t^{2}}{b-a}}\right)}
\end{aligned}
$$

Since the values for $\sigma_{1}$ and $\sigma_{2}$ are not known for the various perspectives, the results presented here, based on parameter distributions, are informed by my "heuristic" attribution of values to perspectives. The results should be interpreted as representing an illustration of the model. The chosen approach, thus, results in somewhat stereotypical perspectives which, nevertheless, demonstrate the implications of differences in perspectives. Moreover, the method can be regarded as an uncertainty analysis in which values of parameters are varied according to the characteristics of perspectives. I will now briefly discuss the preferred values of the distribution function for each perspective as given in Table 7.4. Most of this material is based on Schwartz and Thompson (1990) (See Section 3.3). 


\section{Egalitarians:}

The emphasis placed on equality by egalitarian actors implies that each individual is allowed to emit an equal amount of fossil $\mathrm{CO}_{2}$ emissions annually and will, therefore, presumably allocate rights according to population sizes (high value of $\alpha_{1}$ ). This preference for equality strongly reduces the preference for allocation on the basis of GNP and energy consumption. Egalitarians prefer a long-term horizon for policymaking, as well as being keen to take account of historical inequality. Therefore, high values of $T_{R}-T_{B}$ and $T_{E}-T_{R}$ are preferred. Because all past and future human beings are considered equal, discounting is not regarded as appropriate.

\section{Hierarchists:}

Hierarchists are assumed to be concerned with maintaining institutions and are, therefore, deemed to prefer a balanced mixture of the allocation criteria regarding energy use, GNP and population. The main concern of hierarchists is to maintain the currently functioning system, which influences their attitude towards the distinction between the shorter and ionger term. Climate change discussions are assumed to focus on a long-term period of 40 years for both past and future horizons. Finally, I assume a $2 \%$ discount rate $(\delta)$ for the past and future; this being a moderate pure rate of "social time preference".

Individualists:

Individualists may prefer an allocation based on skills, as measured here by economic output (GNP), and will, therefore, prefer a higher value of $\alpha_{2}$. Furthermore, $\alpha_{1}$ and $\alpha_{3}$ are assumed to be equally weighted, because they are both important inputs for the economic system. The time horizon adopted by individualists tends to be rather short, because they assume that future generations will take care of themselves. Furthermore, they tend to prefer a higher discount rate than the hierarchists, because of their shorter time horizon.

The indicative estimated values of $\sigma_{1}$ for each of the various perspectives are given below. Because the value of $\sigma_{2}$ is unknown, it is assumed to be 50 for all parameters, reflecting a medium degree of preference (see also Figure 7.12). 
Table 7.4: Estimated parameter values $\left(\sigma_{1}\right)$, best guesses, of the allocation function for various perspectives.

\begin{tabular}{|l|c|c|c|}
\hline parameters & individualist & hierarchist & egalitarian \\
\hline$\alpha_{1}$ (population) & 0.1 & 0.333 & 0.8 \\
$\alpha_{2}$ (GNP) & 0.8 & 0.333 & 0.1 \\
$\alpha_{3}$ (energy use) & 0.1 & 0.333 & 0.1 \\
$\delta$ (discount rate) & 0.95 & 0.98 & 1.0 \\
$\mathrm{~T}_{\mathrm{R}}-\mathrm{T}_{\mathrm{k}}$ (past time horizon) & 0 & 40 & 100 \\
$\mathrm{~T}_{\mathrm{k}} \mathrm{T}_{\mathrm{k}}$ (future time horizon) & 10 & 40 & 100 \\
\hline
\end{tabular}

\section{Perspectives-based Scenarios}

Scenarios for future development, which determine the size of the overall $\mathrm{CO}_{2}$ emission budget, will depend, among other things, on the degree of dominance of perspectives. In view of their attitude towards nature conservation, egalitarians can be expected to prefer lower emission levels than individualists. I assume that the IPCC scenarios (IPCC, 1992; see also the Appendix) can be used in a first attempt in representing different perspective scenarios ${ }^{5}$ (utopias). Following on Rayner (1991), I distinguish three scenarios characterized by prevention (egalitarian), control (hierarchist), and adaptation (individualist).

\section{Egalitarians:}

From the viewpoint of egalitarians as defined here, it is morally wrong to distort natural processes. Therefore, a preventive response strategy for a human-induced climate change is preferred. In order to relieve anthropogenic pressure on the environment, growth of economic output should be zero, or very modest at least. Furthermore, the rate of population growth will have to decrease. These conditions will result in a low growth of energy use, and especially of fossil fuel consumption. Among the IPCC scenarios, IS $92 \mathrm{c}$ best meets the above requirernents, since it resuits in a temperature increase of $2^{\circ} \mathrm{C}$ in 2100 (relative to 1900). This is just the minimum target for a low-risk future (AGGG, 1990).

\section{Hierarchists:}

Hierarchists seek to balance economic growth and environmental quality. Such "sustainable" development can be achieved by means of careful stewardship aimed at keeping economic growth within the limits of what nature will allow. A modest

\footnotetext{
s In fact the IPCC scenarios are variations of the hierarchistic perspective. The egalitarian and individualistic scenario chosen seems to include some arguments of egalitarians and individualists.
} 
degree of economic growth is generally preferred in view of the need to feed a growing population. Developments in energy use are determined by technical innovations and the recognition of the exhaustible nature of non-renewable (energy) resources. The IPCC's IS92a scenario, to some extent, shares these assumptions and is, therefore, chosen as the hierarchist scenario.

\section{Individualists:}

Individualists argue that it is morally wrong to restrict economic growth. Nature is assumed to be robust and even in the event of a human-induced climate change, new opportunities might well be created which could stimulate economic behavior. Because (energy) resources are regarded as inexhaustible and nature is expected to be robust, no restrictions on population growth and energy use are set. The IPCC's IS92e scenario is, therefore, the one which will here represent the individualist.

\section{Distribution of Emission Rights}

Given the perspective-based parameter distribution functions and using perspective-dependent future scenarios as defined above, an utopian distribution of emission rights can be derived for future periods. I now propose to discuss the results by reference to allocations for the period 1991 to 2000 . For each perspective, a large sample of 1000 regional emission rights allocations is derived for the period $T_{R}$ (=1990) up to $T_{E}$ (fixed and perspective dependent; $E=2090, H=2030, I=2000$ ). Thereafter, the resulting relative allocations are used to scale the emission rights distribution for the egalitarians and the hierarchists for the period 1991 to 2000 to compare the results of all three perspectives.

The cumulative probability of these allocations is depicted in Figures $7.13 \mathbf{a}, \mathbf{b}+c$. The variances of the regional rights are much smaller than those of a uniform distribution, since the preferences for specific parameter values result in specific allocations. The results set out in Table 7.5 and. Figures $7.13 a, b+c$ show that the distributions of emission rights vary remarkably among the perspectives. From the egalitarian point of view, industrialized regions have emitted such large amounts of fossil $\mathrm{CO}_{2}$ in the last decades that, according to equity principles, they should derive fewer emission rights than the projected level (IS92c) allows them. Consequent trading in emission rights may result in transfers of capital, technology and know-how from "North" to "South" to compensate the developing countries for the "inequitable treatment" in the past.

The allocation of emission rights associated with the hierarchist results in a flow of assets from industrial to developing regions. The magnitude of the flow is smaller than that associated with egalitarian preferences, because the economic system may 188 
become unstable if drastic measures are implemented. Moreover, hierarchists prefer to allocate emission rights by reference to a balanced valuation of equity and economic power. Since hierarchists take historical emissions into account, a surplus of emission rights allocated to developing countries is created.

Although individualists regard measures to reduce $\mathrm{CO}_{2}$ emissions as redundant, I propose to discuss the allocation as preferred by individualists, if a system of tradeable emission rights were, nevertheless, to be introduced. According to the individualist, the high levels of GNP in OECD countries imply that they should be entitled to greater emission rights than emission scenario IS92e allows them. Fossil $\mathrm{CO}_{2}$. intensive economies suffer more than others, if rights are allocated according to an individualist perspective and the result is a lower level of emission rights in EE-CIS and CPA. Although developing countries and EE-CIS are holding about the same share in GNP in the 1990's, emission rights accruing to the "developing" countries are much greater. This is due to assuming (a small degree of) the historical responsibility. The result will be a significant transfer of emission rights in EE-CIS to developing countries (see also Figure 7.10).

Thus, according to the general allocation model, the trading of emission rights seems, in line with the views of egalitarian and the hierarchist, to be preferred. This will result in a flow of assets from "North" to "South". Which combination of parameter values of the allocation model may lead to a politically acceptable allocation depends, among other things, on the willingness to pay from the North and the common opinion about the seriousness of reducing emissions.

Table 7.5: Means of distribution of emission rights (1991 - 2000). Emissions envisaged by the perspective based scenarios are in parentheses. (In GtC for the 10 year period)

\begin{tabular}{|l|c|c|c|c|}
\hline & OECD & EE-CIS & CPA & DC \\
\hline Egalitarian & & & & \\
Emission rights (mean) & 7.4 & 4.0 & 13.1 & 37.9 \\
Emission scenario IS92c & $(28.3)$ & $(15.6)$ & $(7.4)$ & $(11.2)$ \\
\hline Hierarchist & & & & \\
Emission rights (mean) & 26.0 & 8.0 & 9.0 & 25.1 \\
Emission scenario IS92a & $(31 . \mathrm{I})$ & $(16.7)$ & $(8.0)$ & $(12.4)$ \\
\hline Individualist & & & & 1.8 \\
Emission rights (mean) & 43.0 & 6.0 & $(8.5)$ & 20.2 \\
Emission scenario IS92e & $(32.2)$ & $(17.3)$ & $(13.0)$ \\
\hline
\end{tabular}



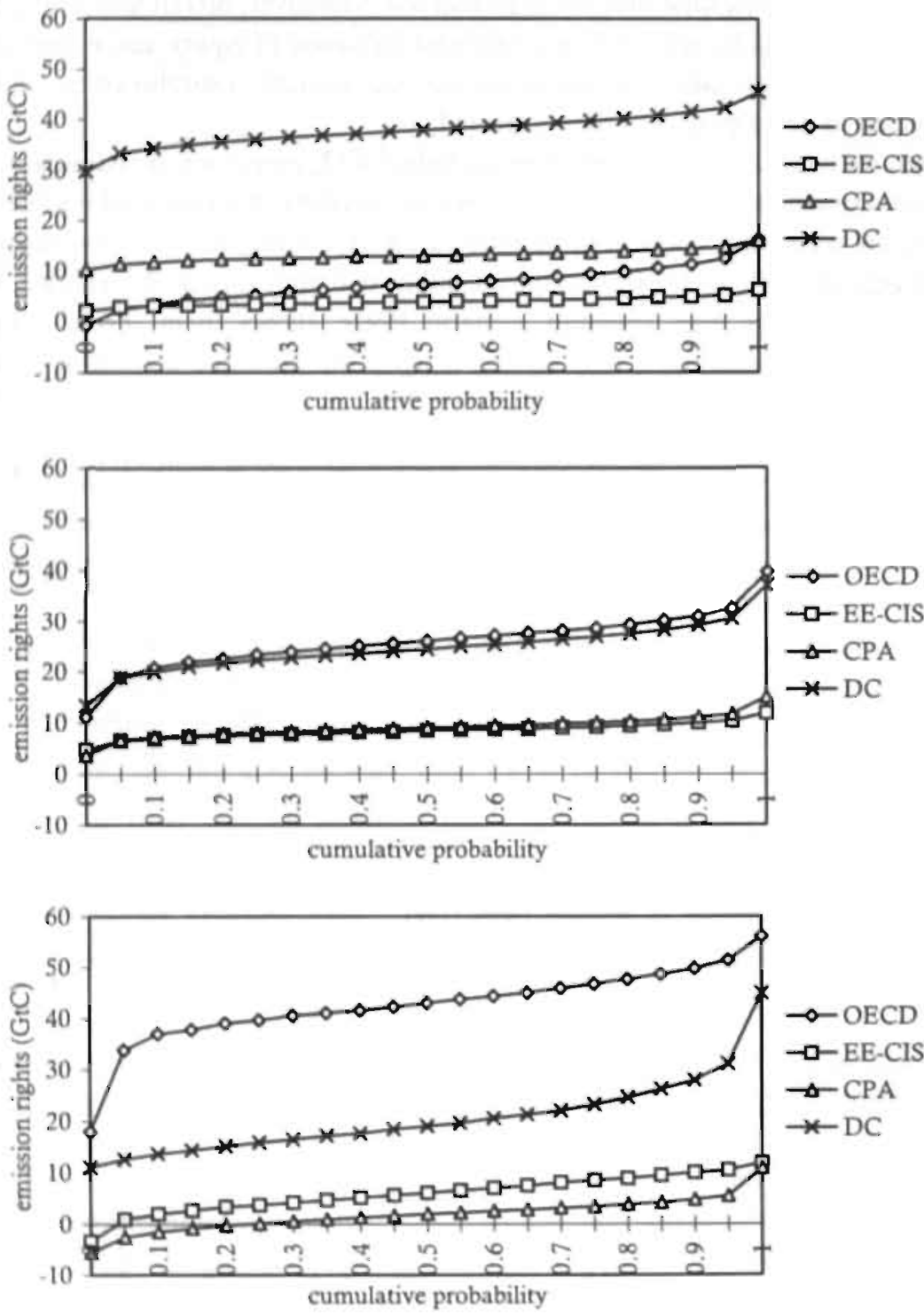

Figure 7.13a,b,c: Cumulative probability of regional fossil $\mathrm{CO}_{2}$ emission rights from an egalitarian, hiearchistic and individualistic perspective. 


\subsubsection{Scenarios of Perspective Change}

Agents' perspectives may change as they are faced with a persistent pattern of surprises. In Chapter 8, a broad discussion of perspective changes is made and an evolutionary model to simulate such dynamics is introduced. In this Section, simple scenario based exercises are performed to analyze the influence of changes in perspectives by two scenarios in which the dominant attitude towards the climate change problem changes. Since the policy makers considered in these scenarios represent the world community, global scenarios of future developments can reasonably be expected to change in the course of time. The scenarios envisage transitions from egalitarian to individualistic management style and vice versa.

"Transition from individualistic to egalitarian". The individualist scenario is traced for the period 1991 - 2020, because there is no shared belief in the existence of a real risk. In the years following, the perspective is to become hierarchical in character, since the first effects of climate change become apparent around that date. In about 2050, the new dominant perspective is egalitarian in response to scientific evidence of largescale anthropogenic influence on the global climate.

"Transition from egalitarian to individualistic". The egalitarian scenario is traced for the period 1991 - 2020 because decision-makers aim at minimizing the risks of human-induced climate change. After 2020, the new perspective is hierarchist because no convincing scientific evidence for a human-induced climate change can be found. Around 2050, the new perspective changes to that of the individualist because the hypothesis of a human-induced climate change is rejected.

Where $T_{R}$ is equal to $1990,2000, \ldots, 2100$, emission rights are allocated according: to the dominant perspective at that particular moment in time. The future scenarios, for population size, GNP, energy use, and emissions are derived by reference to the growth rates associated with the perspective scenarios which are dominant. Mean values of the emission right distributions are derived using the corresponding $\sigma_{1}$ values themselves. The relative regional shares are used to allocate the expected emissions for the years $\mathrm{T}_{\mathrm{R}}$ (Figures 7.14a,b). 

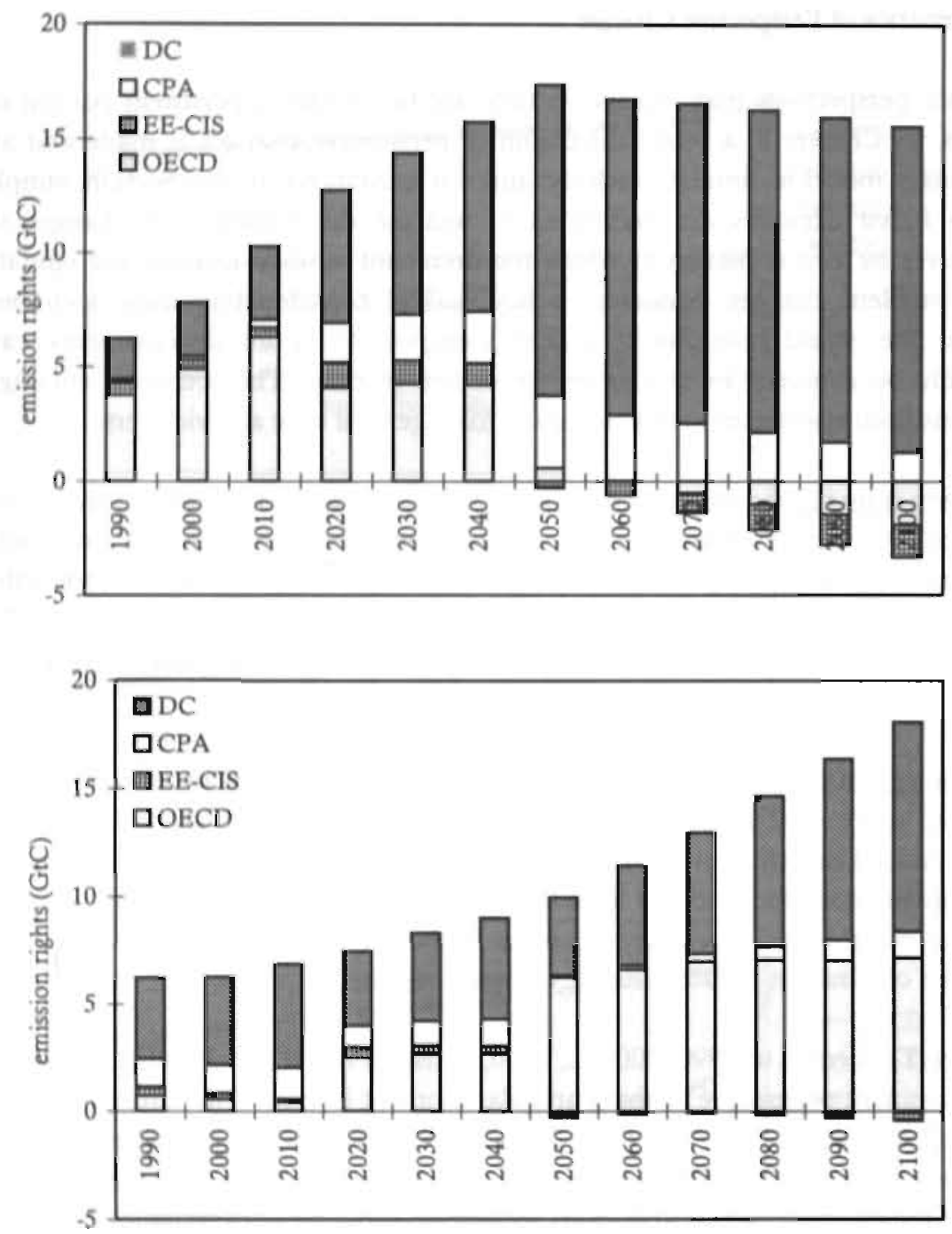

Figure 7.14a,b: (A) Regional emission rights of a "transition from individualist to egalitarian" scenario. (B) Regional emission rights of a "transition from an egalitarian to an individualistic"scenario". 
According to the "transition to egalitarian" scenario, global emissions initially rise rapidly, as is consistent with the individualist scenario. According to the individualist, there is no need to allocate emission rights at all during this period. With the transposition to a hierarchical perspective, the OECD countries forfeit emission rights in favor of (formerly) centrally-planned regions in the years following 2020. In view of high emission levels over several decades, the influence of human activities on the climate system becomes apparent and results in policy changes after 2050, according to the dominant egalitarian view. Because the population continues to grow at a rapid rate up to 2050 , the emission rights of developing regions jump sharply as the dominant perspective changes. However, by that stage, the cumulative contemporary and historical contributions in the industrialized regions have reached a level at which emission rights have expired. This scenario, thus, suggests that playing down the possibility of an anthropogenic climate change becoming a problem creates a hostage to fortune. In the long run, the industrialized countries, and especially the $\mathrm{OECD}$ members, will pay dearly in the loss of fossil $\mathrm{CO}_{2}$ emission rights, if they are forced to admit that a problem exists.

According to the "transition to individualistic" scenario, the increase of global emissions in the coming decades is limited because of preventive policies, according to the egalitarian perspective. The emission rights of developing regions are higher than in the emission scenario, while those allocated to industrialized regions are lower. The egalitarian perspective anticipates the exchange of surplus emission rights through international transfers between industrialized regions and developing regions. However, as soon as the dominant perspective gives way to hierarchist thinking, global emissions start to increase once again. The emission rights of developing regions are curtailed and those of industrialized regions increase. As soon as the hypothesis of the possibility of a human-induced change is rejected, development will be unrestricted and there will be no reason for an allocation mechanism.

Limiting emissions in the absence of human-induced climate change may have negative consequences for global economic development in the short term. However; in the long term the same levels might be reached as in the "transition to egalitarian" scenario. Moreover, (technological) transactions from "North" to "South" may cause economic growth to be enhanced, especially in developing regions.

The scenarios tracing changing perspectives demonstrate that it is difficult for a region to determine its strategy. The failure to take measures in the present, may, if climate change does occur, in the long run be advantageous to developing countries, in the light of the initial allocation of emission rights. On the other hand, taking measures in case of a rejection of the hypothesis of a human-induced climate change may be advantageous to developing regions, in the short term. The position of OECD 
countries is precisely the opposite, while neither scenario envisages more than modest emission rights being allocated to EE-CIS countries. This state of affairs underlines the delicate international economic position of the EE-CIS region.

\subsection{Conclusions}

The industrialized countries have so far caused the major part of $\mathrm{CO}_{2}$ emissions. Analyzing past carbon emission from fossil fuel use, cement manufacturing, and land. use change indicates that present wealth in the industrialized countries has been at the cost of large emissions in the past. The relative contribution of Western Europe and North America to the $\mathrm{CO}_{2}$-concentration rise over the period 1800 to 1990 is about $40 \%$, almost completely due to fossil fuel combustion and cement manufacturing. The contribution from Africa, Latin America and South/South-East Asia, exceeding 30\%, is for about $75 \%$ due to deforestation.

From an egalitarian perspective, the industrialized world should take prime responsibility in responding and supporting the developing countries in contributing their share to mitigating climatic change without thwarting their development. As a consequence, the international policy discussion understandably adds the economic North-South issues to the search for technical options. A reduction of the risk of adverse climate change according to the "precautionary principle" approach implies that furure global emissions of greenhouse gases are constrained.

Under the premises of a future scenario (for example IS92C), the world would be constrained by the global carbon budget, defined as the cumulated $\mathrm{CO}_{2}$ emission from fossil fuel combustion, cement manufacturing, and land use change over the period. 1800 to 2100 , of about $1130 \mathrm{GtC}$. About $30 \%$ of this budget has already been used up in the past. If all world citizens living in the period 1800-2100 were allocated an equal emission quotum, this would result in a per capita emission of $0.97 \mathrm{tC} /$ cap yearly. The industrialized regions would have exceeded their allocated budgets for 1800-1990 by about $87 \mathrm{GtC}(\mathrm{OECD})$ and $16 \mathrm{GtC}$ (CIS). The developing countries, however, have an emission credit of about $130 \mathrm{GtC}$.

A more sophisticated allocation model is presented which takes into account historical and future developments. Criteria used in allocation are population size (equity per capita), GNP (economic affluence), energy use (efficiency), the length of historical responsibility, and time preference.

There are various perspectives pertaining to the allocation of a constrained global fossil $\mathrm{CO}_{2}$ emission budget. In the first place, there might be differences in the scenarios concerning fossil $\mathrm{CO}_{2}$ emissions, energy use, population, technology and economic growth. Second, the preferred value distribution of parameters of the model 
might be skewed by perspectives. Drawing on Schwartz and Thompson (1990), the perspectives are labelled "individualist", "hierarchist" and "egalitarian", and are represented by distributions of preferred values of parameters in the allocation model and by various preferred future scenarios.

The method can be interpreted as an example of a quantification of subjective judgment which is intended to take account of perspectives affecting the allocation of emission rights. The resulting three perspective-based allocations of emission rights span a much wider range of possible allocations than other studies so far. It demonstrates the importance of the influence of subjectivity on the ranges of the initial allocation of emission rights. Because of the political dimension of the subject and the dynamic shifting of the mixture of perspectives in time, the results show that there is no optimal allocation of emission rights.

A logical follow-up of this study is to use a regionalized energy model to analyze the effectivity of various initial emission right allocations and their trade schemes on the ability to meet global policy targets on climate change. Next to official IPCC scenarios, target-scenarios, as will be determined similar to the approach in Chapter 6, may then be used to calculate regional emission debts and rights. I hope that scanning the impact of the various distributions may help the discussion on sharing responsibility to meet emission targets.

A regionalized version of the energy model as used in Chapter 6 is under way, including a trade model for energy carriers (Targets-IMage Energy model Regional - TIMER). 
Appendix: Summary of Assumptions in the IPCC 1992 Alternative Scenarios (IPCC, 1992).

\begin{tabular}{|c|c|c|c|c|c|c|}
\hline Scenario & Population & Econcmic & Growth & Energy Supply & fther & CFa \\
\hline IS92: & $\begin{array}{l}\text { World Bank } 1991 \\
\text { II 3B by. } 2100\end{array}$ & $\begin{array}{l}1990-2025 \\
1990-2100\end{array}$ & $\begin{array}{l}2.9 \% \\
2.3 \%\end{array}$ & $\begin{array}{l}12,000 \text { EJ Conventional Oil } \\
13,000 \text { EJ Natural Gas } \\
\text { Solar costs fall to } 50.075 / \mathrm{kWh} \\
191 \text { El of biofuels avaliable at } \\
570 / \text { barrel }\end{array}$ & $\begin{array}{l}\text { Lowlly enacted and } \\
\text { inemationally apreed cuntrobs on } \\
\text { Shx, NOx and NMVOC } \\
\text { enignions }\end{array}$ & $\begin{array}{l}\text { Partial compliance with } \\
\text { Montreal Protocol. } \\
\text { Tectnolopical transfer results in } \\
\text { gradual ptase out of CFC also } \\
\text { in non-signatory countries by } \\
2075 \text {. }\end{array}$ \\
\hline $1592 \mathrm{~b}$ & $\begin{array}{l}\text { World Banik } 1991 \\
113 \mathrm{Bby} 2100\end{array}$ & Same as "a" & & Same as " $\mathrm{A}$ " & $\begin{array}{l}\text { Sime as " } 2 \text { " phus commitmens by } \\
\text { many } \mathrm{OECDD} \text { countries to sabilize' } \\
\text { ereduce } \mathrm{CO}_{2} \text { emissions }\end{array}$ & $\begin{array}{l}\text { Giotual compliance with } \\
\text { scheduled phase out of Montreal } \\
\text { Protocol }\end{array}$ \\
\hline $\mathrm{ISP}=\mathrm{C}$ & $\begin{array}{l}\text { UN Medium } \\
\text { Low Case } \\
6.4 \mathrm{~B} \text { by } 2100\end{array}$ & $\begin{array}{l}1990-2025 \\
1990-2100\end{array}$ & $\begin{array}{l}2.0 \% \\
1.2 \%\end{array}$ & $\begin{array}{l}8,000 \text { EJ Conventional OA } \\
7,300 \text { EJ Natural Gas } \\
\text { Nuclear costs decline by } 0.4 \% \text { annually }\end{array}$ & Sme as " a" & Same as " $\mathrm{a}$ " \\
\hline $1592 \mathrm{~d}$ & $\begin{array}{l}\text { UN Medium } \\
\text { Low Cine } \\
64 \text { B by } 2100\end{array}$ & $\begin{array}{l}1990-2025 \\
1990-2100\end{array}$ & $\begin{array}{l}2.7 \% \\
2.05\end{array}$ & $\begin{array}{l}\text { Oil and gas as "C. } \\
\text { Solar costs fall in } 50.065 / \mathrm{kWh} \\
272 \text { EJ of biofuels available at } \\
550 / \text { barrel }\end{array}$ & $\begin{array}{l}\text { Enission controls extended } \\
\text { Forddwide for } \mathrm{CO}, \mathrm{NOx} \text {, } \\
\text { MMrVOC and } \mathrm{SOx} \text {. Halt } \\
\text { dforestation: Capture and we of } \\
\text { elissions from coal mining and } \\
\text { ges production and use. }\end{array}$ & $\begin{array}{l}\text { CFC production phase out by } \\
1997 \text { for industrialized coumtries. } \\
\text { Phaxe out for HCFCs }\end{array}$ \\
\hline Ispe & $\begin{array}{l}\text { World Bank } 1991 \\
113 \text { B by. } 2100\end{array}$ & $\begin{array}{l}1990-2025: \\
1990-2100:\end{array}$ & $\begin{array}{l}3.5 \% \\
3.0 \%\end{array}$ & $\begin{array}{l}18,400 \text { EJ Conventional Oil } \\
\text { Gas same as " }{ }^{*} \\
\text { Phase out nuclear by } 2075\end{array}$ & $\begin{array}{l}\text { Enission controls ( } 30 \% \text { pollution. } \\
\text { Encharge on fossil energy) }\end{array}$ & Same as " $d$ " \\
\hline ts92f & $\begin{array}{l}\text { UN Medium } \\
\text { High Case } \\
17.6 \mathrm{~B} \text { by } 2100\end{array}$ & Same as "a" & & $\begin{array}{l}\text { Oil and gas same as "e" } \\
\text { Solar costs fall to } 50.08 .3 / \mathrm{kWh} \\
\text { Nudear costs increase to } 50.09 / \mathrm{kWh}\end{array}$ & Sime as ${ }^{\circ}{ }^{*}$ & Same as " $\mathrm{a}$ " \\
\hline
\end{tabular}




\section{8}

\section{The Battle of Perspectives ${ }^{1}$}

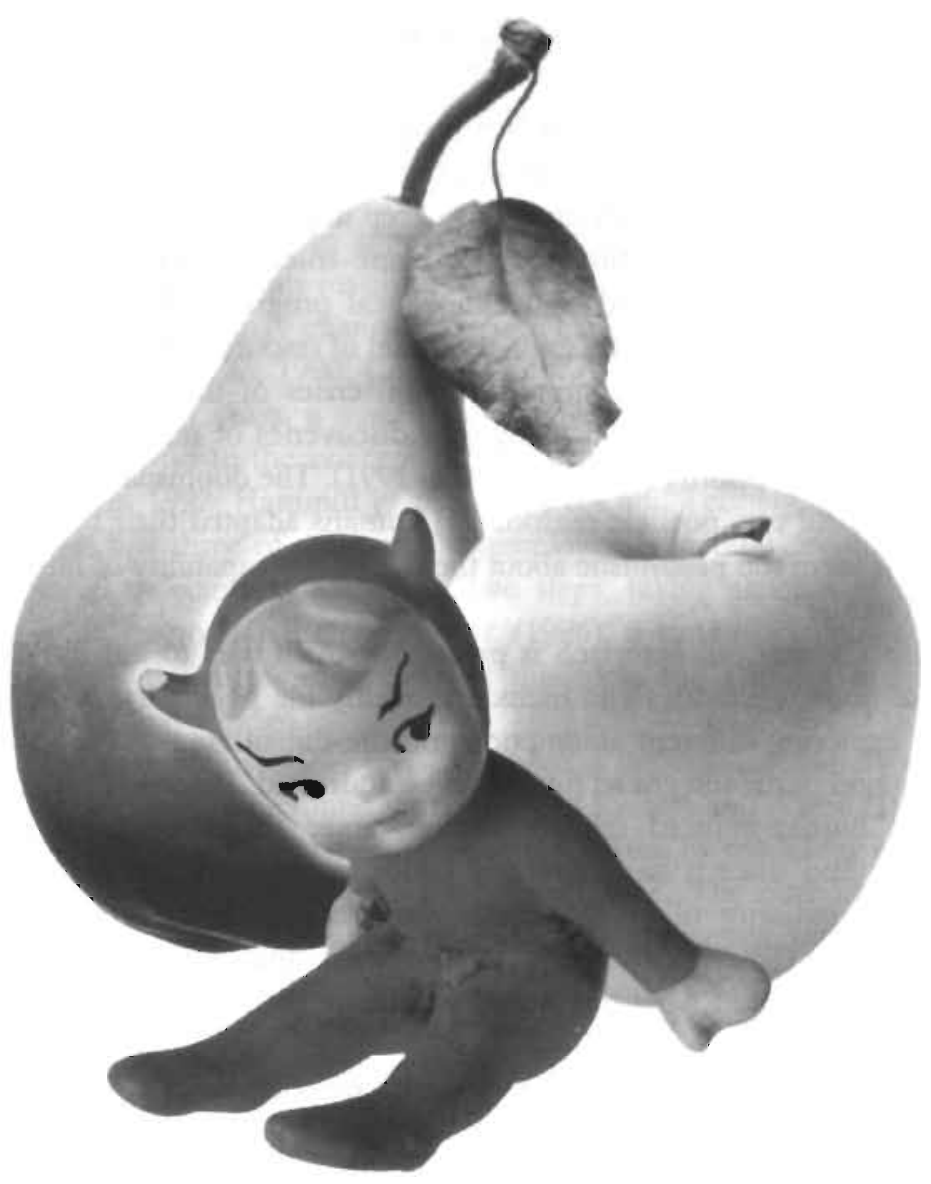

'This Chapter is based on Janssen (1996c). 


\subsection{Introduction}

Scanning the future raises the problem of assessing inherently unpredictable and, therefore, highly uncertain phenomena. Policies are often made in the face of ignorance about the actual dynamics of the system. This ignorance may stem from the fact that the requisite knowledge lies in the future (De Greene, 1993). Needless to say, policies are value-oriented, values change over time, and knowledge is never perfect at any given time.

Two examples of outdated predictions are the following. About two centuries ago, Malthus (1798) regarded food production as a land-limited resource that could not possibly be increased quickly enough to keep in pace with a growing population. Malthus's pessimistic expectation did not come true, due to a sharp increase in agricultural productivity caused by technological progress. Meadows et al. (1972) presented the waming that the ongoing depletion of resources would result in a world wide collapse of the world economy. The oil crises of the 1970's resulted in an increase of energy efficiency and many new discoveries of reserves placed resource depletion at a lower priority (Meadows et al., 1991). The doomsday scenarios did not become reality for two possible reasons: (1) humans adapted their behavior, and (2) the scenarios seem too pessimistic about the innovative capability of humans and the capacity of nature.

In this Chapter, an approach is presented which tries to include the adaptive behavior of human activities and include explicitly the possibility of different world views. By exploring different assumptions on the functioning of the global system, different images of the integrated future can be addressed. Proposed is to focus on the problem of human-induced climate change, since this problem is not without controversies and since it has important policy implications for the coming years. Although the majority of climate researchers support the theory of the natural greenhouse effect (IPCC, 1990), it is unclear where and how fast such temperature increases will occur and what the impact will be on the biosphere, including human society.

Those engaged in climate change research are continually confronted with new surprises. For example, in recent years new scientific findings (IPCC, 1992; Schimel et al., 1994), have shown that: (a) negative radiative forcing due to ozone depletion could counteract positive radiative forcing associated with chlorofluorocarbons (CFCs); (b) there is a possible cooling effect due to aerosols resulting from sulphurous emissions; (c) the rates of increase in the atmospheric concentration of most greenhouse gases have slowed down; (d) recent measurements of the $\mathrm{CO}_{2}$ in the atmosphere show a 
levelling off of the concentration, while paradoxically the emission rate has not stabilized at all.

The familiar approaches which employ integrated assessment models are scenario analysis and optimization. Scenario analyses may be seen as a means of scanning possible future developments, although the scenarios tend to lose their meaning, if agents do not learn from, and react to, surprises. Optimization, on the other hand, is based on the assumption that rationally-acting agents have perfect knowledge about the system in question and are able to determine the optimal strategy for the next century. Although this approach may yield valuable insights into efficient strategies, it can never arrive at optimal solutions to problems which arise, and probably will continue to arise, as researchers are confronted with new scientific insights,

Thus, there is a need for an approach which is able to address the adaptive behavior of the numerous agents involved in climate change policy. The recognition that the behavior of agents may change over time is not new in modelling the global system (e.g. Thompson et al., 1990; Costanza et al., 1993; Holling, 1994; Holling et al., 1995). During recent years, a number of studies related to climate change have appeared which investigate the concept of adaptive or sequential decision-nnaking (Manne and Richels, 1992; Hammitt et al., 1992; Peck and Teisberg, 1993b; Lempert et al, 1996). The first three of these studies employ a sequential-decision model in which the optimal trajectory is derived in two steps, taking account of a learning phase in the initial period. In Lempert et al. (1996), a simple adaptive strategy is examined, whereby different assumptions of the costs and damage functions are juxtaposed with optimal policies.

Among the attempts to model social behavior is the artificial society approach. An interesting example is to be found in the work of Epstein and Axtell (1995), since they designed a program which generates artificial societies by modelling simple rules for hundreds of individual agents who evolve over time. The purpose of such an artificial society is to enable the investigation of social processes within a so-called "CompuTerrarium".

However, these models do not take into account the diversity of world views among the agents which may influence those agents. Thompson et al. (1990) argue that agents are forced to cast around for alternatives in the event that they are confronted with a persistent pattern of surprises, and a number of studies have tried to model such changing perspectives.

Thompson and Taylor (1986) devised a computer simulation of a so-called "surprise gamen in which a so-called payoff matrix is used to value the "nice" and 
"nasty" consequences of various surprises. The game was implemented for an imaginary industrial enterprise with a considerable number of competitors, and simulates the number of egalitarians, hierarchists, individualists, and fatalists in time.

In a set of exploratory experiments, Janssen and Rotmans (1995) generated various scenarios in which climate policy is changed as a result of shifts in the dominant perspective among the agents. The dominant perspective may change, if expectations about the functioning of the system fail to correspond with reality. The study assumed that scenarios would involve change and did not simulate the underlying dynamics forcing agents to change their perspectives, which is the aim of this Chapter.

Learning and adaptation is assumed to be simulated by changes in agents' perspectives, and where these changes are caused by the imperfect description of agents' expectations of the system and its observations. I recognize that this is a highly simplistic representation of reality, but am convinced that it, nevertheless, enables me to start implementing the concept of agents with adaptive behavior and diverse perspectives of the world in integrated assessment modelling.

\subsection{The Concept}

The aim of the "Battle of Perspectives" is to simulate changing world-views of the agents which are assumed to change the behavior of those agents. The agents are assumed to represent abstract images of decision-makers on an international level and, thus, could ideally be thought of as institutional actors. The dominant perspective among the agents evolves over time and is fed by the agents' observations of the system. In a "competitive" environment, in which adherents to a variety of perspectives all claim to provide explanations, agents try to find the best possible explanation of the observations: hence the "Battle of Perspectives" (Figure 8.1).

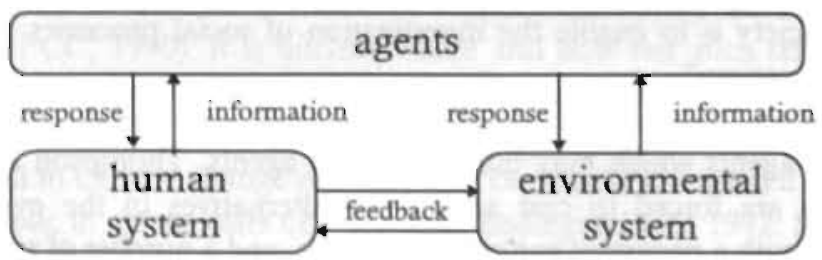

Figure 8.1: The Battle of Perspectives: The integration of (behavior of) agents with the human and environmental system. 
The agents are simulated as a complex adaptive system (see Chapter 3). In this Chapter, the agents differ in their world views and, therefore, their preferred management style. Their success in explaining the information derived from the system determines the fitness of the agents' world view. The better the agent's world view explains observed behavior, the higher the chance that the agent will not change its world view. On aggregate, there is a trend to change to the world view which explains the observations in the most likely way. Note that the agents represent decision makers and not arbitrary individuals.

As a framework to classify possible world views, three "active" perspectives are distinguished based on the Cultural Theory (see Chapter 3). Although such a classification consists of stereotypes, it might nevertheless prove to be useful in analyzing the dynamics of behaviour associated with changes in the perspectives adopted by agents. The active perspectives are used as extremes of a continuum which is used to describe all possible points of world views. The agents' perspectives are depicted in this triangle and swarm over the continuum fed by the information from the system (Figure 8.2).

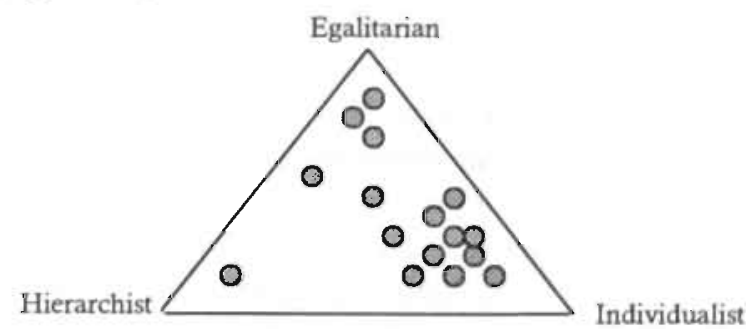

Figure 8.2: Agents's perspectives located within the perspective triangle

A widely used approach in scanning the future is the use of utopias and dystopias. A utopia describes the preferred developments according to a certain development, while a dystopia describes what happens if the preferred "policy" does not fit with the functioning of the global system. The doomsday scenarios of Malthus and Meadows et al. (1972) can be seen as dystopias, where the trends in human activities were assumed not to fit with nature's capacity. Note that the scenario which avoids a collapse (Meadows et al., 1972) can be seen as a utopia. A dystopia scenario for climate change is the "Business-as-Usual" scenario of the IPCC (1992), where the emissions will cause an excess of environmental quality targets, if no additional policy is implemented. 


\section{MEETING TARGETS}

Trisoglio et al. (1994) characterize the perspectives by the following two dimensions: (1) how is the world seen; and (2) the preferred management style. The combination of each perspective's world view with its respective favored strategy is used to assess the utopias (Trisoglio et al., 1994). Agents' utopias are used to describe the management style which corresponds with the agents' view of the world. The dystopias describe what would happen to the world if the agents' favored strategy were to be applied, while reality seems to follow another world view. The utopia/dystopia approach as adopted by Trisoglio et al. (1994) and Van Asselt and Rotmans (1996) is essentially static. Their approach relies upon an agents' current conception of future, which, in turn, determines the present policy which agents might adopt in the form of a set of rules which guide decision-making. In this Chapter, an attempt is made to simulate the agents' perspectives in time, so the manner in which the agents learn is modelled, so that they change their opinions and adapt in the face of surprises, and respond to new evidence which will surface in a changing system.

Translated to the Battle of Perspectives the four phase cycles of Holling (Section 3.4) explains that surprises may lead to a loss of control which provide the proximate trigger for change. When agents are confronted with the fact that current interpretations of observations fail, they may benefit to search for more satisfactory explanations. A new perspective become dominant leading to a new stable situation. The related changes in agents' behavior may lay in the structure of the existing social dynamics. However, reshaping of norms and values may cause an increasing pressure to flip the behavior dynamics to a new kind of system. In the presented prototype I only consider one adaptive cycle in the climate policy. This means that only one transition may occur in the dominating position within the perspective triangle, and that the corners of the triangle do not change in time.

\subsection{The Model}

For the purpose of this study, a simple dynamic system is employed which describes the basic dynamics of the economy and the climate system. The model is based on existing economy-climate models, such as those found in: Nordhaus (1992; 1993; 1994), Manne et al., (1994), Hammitt et al. (1992) and Lempert et al. (1996). I recognize that this model yields a highly simplified representation of the problem, but: its dynamic framework serves well to illustrate the "Battle of Perspectives". 
Economic output $\mathrm{Y}(\mathrm{t})$ is given by a standard constant-returns-to-scale CobbDouglas production function in the levels of technology $a(t)$, capital $K(t)$ and labor, which is assumed to be proportional to population, $\mathrm{P}(\mathrm{t})$, for which a future projection published in Bulatao et al. (1990) is used, while $\gamma$ represents the elasticity of output with respect to capital which is taken to be 0.25 . The impact of emission reductions and global climate change on output is represented by the scale factor $\mathrm{S}(\mathrm{t})$.

$$
Y(t)=S(t) \cdot a(t) \cdot K(t)^{\gamma} \cdot P(t)^{t \gamma}
$$

Technological changes are regarded as being exogenous, although such development

$$
\frac{d a}{d t}=\exp \left(\delta_{a}^{a n t} \cdot t\right) \cdot a, \quad \frac{d \delta_{a}^{\text {ant }}}{d t}=-\delta_{a} \cdot \delta_{t}^{\text {nat }}
$$

is assumed to proceed at a declining rate

Total consumption $\mathrm{C}(\mathrm{t})$ is equal to economic output minus gross investments, where $\mathrm{I}(\mathrm{t})$ is the fraction of the economic output which is reinvested in capital stock.

$$
\begin{aligned}
& C(t)=(I-I(t)) \cdot Y(t) \\
& \frac{d K}{d t}=I \cdot Y-\delta_{k} \cdot K
\end{aligned}
$$

The capital balance equation for the capital stock $K(t)$ is defined in terms of investments minus depreciation:

where $\delta_{k}$ is the rate of depreciation of the capital stock, i.e. $10 \%$ per annum, reflecting an average lifetime of capital of ten years on a declining balance method.

The level of fossil $\mathrm{CO}_{2}$ emissions is the result of: the transition towards alternative fuels, $\mathrm{M}(\mathrm{t})$, the energy conservation transition, $\sigma(\mathrm{t})$, economic output, $\mathrm{Y}(\mathrm{t})$ and a coefficient $\alpha(=0.32 \mathrm{GtC} /$ bil $\$)$ :

$$
E(t)=\alpha \cdot M(t) \cdot \sigma(t) \cdot Y(t)
$$

where $\mathrm{M}(\mathrm{t})$ is one minus the percentage of emission reductions and is defined as a logistic function, where $\mathrm{R}$ is the number of years which would be required to reduce the share of fossil fuels within the energy mix by $50 \%$, and $\varepsilon$, in conformance with Lempert et al. (1996), is taken as being 0.01, reflecting an autonomous trend in decarbonization. 
Thus:

$$
\begin{gathered}
M(t)=\frac{1}{I-\varepsilon} \cdot \frac{1}{I+\exp \left(\rho_{M}(t) \_\left(\frac{R(t)}{R(t-I)}(t-1995)-R(t)\right)\right)} \\
\text { where } \rho_{M}(t)=\frac{-I}{R(t)} \cdot \ln \left(\frac{\varepsilon}{I-\varepsilon}\right)
\end{gathered}
$$

The energy intensity decline is described by a logistic fisnction, where $\delta$ is the contribution of available low-cost conservation measures and where the number of years required to double energy efficiency is assumed to be 50 years:"

$$
\sigma(t)=(l-\delta)+\frac{l}{l-\varepsilon} \cdot \frac{\delta}{l+\exp \left(p_{\sigma} \cdot(t-1995-50)\right)}
$$

$$
\text { where } p_{\sigma}=\frac{-l}{50} \cdot \ln \left(\frac{\varepsilon}{1-\varepsilon}\right)
$$

The atmospheric $\mathrm{CO}_{2}$ concentration $\left(\mathrm{pCO}_{2}\right)$ is modelled using the reduced-form carbon cycle model developed by Maier-Reimer and Hasselmann (1987). Carbon emissions (E) are divided into 5 classes (fractions $c_{1-5}$ ), four of which have different atmospheric lifetimes $a_{14}$. Thus:

$$
p C O_{2}(t)=p C O_{2}\left(t_{0}\right)+\int_{t_{0}}^{t} 0.47 \cdot E(\tau)\left(c_{l}+\sum_{i=2}^{s} c_{i} \cdot \exp \left[\frac{\tau-t}{a l_{i-l}}\right]\right) d \tau
$$

where $c_{1.5}=0.13,0.20,0.32,0.25$, and 0.1 , and $\mathrm{al}_{14}=363,74,17$, and $2 \mathrm{yrs}$.

Equation (8.8) was fitted by least squares to the computed response of a full-scale ocean carbon cycle model and yields a good approximation for small changes, although it underestimates the amplitude and time-scale of the response for higher concentration levels (Maier-Reimer and Hasselmann, 1987). 
The radiative forcing of $\mathrm{CO}_{2}$ is modelled in conformance with the IPCC (1990) in the following way:

$$
\Delta Q_{\mathrm{Co}_{2}}(t)=\frac{\Delta Q_{2 \times \mathrm{COO}_{2}}}{\ln (2.0)} \cdot \ln \left(\frac{p C O_{2}(t)}{p C O_{2}\left(t_{0}\right)}\right)
$$

where $\Delta \mathrm{Q}_{2 \mathrm{rCO}}$ is the radiative forcing associated with a doubled $\mathrm{CO}_{2}$ concentration $\left(4.3 \mathrm{~W} / \mathrm{m}^{2}\right)$

Aggregated radiative forcing is assumed to have the following impact on the change in the global mean surface temperature $\left(\Delta T^{\top}\right)$ :

$$
\Delta T^{p}(t)=\frac{\Delta T_{2 x \mathrm{COI}_{2}}}{\Delta Q_{2 \mathrm{xCO2}}} \cdot \Delta Q_{\mathrm{CO2}}(t)
$$

where $\Delta \mathrm{T}_{2 \times \mathrm{CO}_{2}}$ is the global mean surface temperature change in the event of a doubled $\mathrm{CO}_{2}$ concentration (best guess $2.5^{\circ} \mathrm{C}$, see Section 8.5.2).

Since oceans take a long time to warm up, the actual temperature increase $(\Delta T)$ will lag behind the potential increase, which is modelled as follows:

$$
\frac{d \Delta T}{d t}=\beta \cdot\left(\Delta T^{p}-\Delta T\right)
$$

where $B$ is assumed to be 0.05 .

The scaling factor $S(t)$ is the ratio of one minus the percentage of abatement costs to one plus the percentage of damage costs. The (market) damage costs are quantified as a relation between global temperature $\Delta T(t)$ increase and income loss, where $\theta_{1}$ represents the scale of damage: and $\theta_{2}$ the non-linearity in the damage function. The costs of reducing emissions of greenhouse gases are related to (1-M), the fractional. reduction of greenhouse emissions, while $b_{1}$ and $b_{2}$ represent the scale and non-linearity of the cost function. Thus:

$$
S(t)=\frac{l-b_{l} \cdot(l-M(t))^{b_{2}}}{l+\theta_{l} \cdot \Delta T(t)^{\theta_{2}}}
$$




\subsection{Modelling Learning and Adaptive Behavior}

\subsubsection{Introduction}

With respect to all the model's limitations, the agents are assumed to be abstract images of decision-makers on an international level, responsible for climate change policy and able to determine the level of investments and emission reductions. Each agent adheres to a world-view which is located within the perspective triangle (Figure 8.2). By considering a set of similar agents who adopt various perspectives with respect to the climate change problem, the model might be able to simulate a learning process for the agents and their adaptation of their behavior in terms of policy measures, Of course, I would not claim that empirical agents actually learn and adapt in a way which closely resembles the "Battle of Perspectives". I do, however, conjecture that there might be a sort of "weak isomorphism" between the "Battle of Perspectives" and the ways in which actual agents adapt to their changing environment.

Although the perspectives of the agents may change over time if they are confronted with surprises, the underlying system is assumed to follow the assumptions of one of the three extreme perspectives. The world-views of the adherents to the three active perspectives are first discussed, and then the management styles as adopted by the agents concerned are reviewed. The Section is concluded with a discussion on the modelling of the changes in perspectives.

\subsubsection{World View}

Taking the model described in Section 8.3 as representing the global system, the values of several parameters within the model are assumed to vary according to the three perspectives. In conformity with the construction of the uncertainty space in Lempert et al. (1996), and sensitivity analysis in Nordhaus (1994), the following issues are distinguished as being subject to uncertainty:

- climate sensitivity,

- technological improvements,

- mitigation costs,

- damage costs due to a climate change. 
Choices are mainly based on the work of Schwartz and Thompson (1990), Rayner (1991) and Van Asselt and Rotmans (1996). It should be stressed that only parameter values are changed, although differences in perspectives principally also affect the mathematical system. For practical reasons, this is hitherto excluded.

\section{Individualists}

According to the individualists' view of a benign natural system, climate change will be mitigated by known and hitherto unknown dampening feedbacks. Speculative negative feedbacks are, therefore, taken into account, whereas uncertain positive feedbacks are neglected or considered to have negligible impacts on the climate system. Consistent with the lowest estimate of $\Delta \mathrm{T}_{2 \mathrm{xcos}}$ in the literature, a value of $0.5^{\circ} \mathrm{C}$ for $\Delta \mathrm{T}_{2 \mathrm{x}} \mathrm{cos}$ is adopted (Lindzen, 1990 ).

Individualists, believing in a stable system, assume that no economic damage will be suffered as a result of climate change. In the event that a climate change should occur, technical solutions will balance out any negative effects.

Technologies which have been created in the past extended life expectancies and enhanced affluence, bringing with them, in addition, incremental pollution, technological risk and climate change. On the other hand, technological development also generates numerous solutions for dealing with the unwanted by-products. In choosing the parameter values, I assume that the individualists are desirous of an appropriate technological development which is as cheap and advancing as possible, and, therefore, a high rate of technological development is assumed. However, on the other hand, individualists assume that the amount of low-cost conservation of energy is low, while the market will not stimulate this kind of development. Their self-interest and short-term reasoning imply a low rate of technological progress in finding expensive ways to decarbonize economic production.

\section{Hierarchists}

Hierarchists are assumed to interpret uncertainties in a similar manner to prominent scientific experts and institutions (such as the Intergovernmental Panel on Climate Change (IPCC)). The central estimate of the IPCC, $2.5^{\circ} \mathrm{C}$, for $\Delta \mathrm{T}_{2 \times \mathrm{CO} 2}$ (IPCC, $1990 ; 1992$ ) is adopted, which is consistent with their trust in institutions; hierarchists, thus, are assumed to follow the best-guess estimates of the scientific community. Following Nordhaus' (1994) central estimate, a quadratic relation between temperature increase and damage cost results in a $1.3 \%$ loss of economic growth if temperature increases by $3^{\circ} \mathrm{C}$. 
Expectations of technological progress which stimulates economic development are based on technological improvement rates in the past. In balancing the risks, hierarchists stimulate research for alternative solutions. The hierarchist is assumed to stimulate decarbonization of economic production and, therefore, a moderate value of $\mathrm{d}$ is assumed. Furthermore, a best-guess estimate of the mitigation cost is assumed as used by Nordhaus (1994) who based his estimates on a survey of energy models (Nordhaus, 1991b).

\section{Egalitarians}

The egalitarian myth of nature suggests that minor changes disproportionately influence the behavior of the system. Consistent with egalitarians' view of climate change as a catastrophic threat, they consider all uncertain processes and feedbacks as having amplifying effects on the human-induced disturbance of the global climate. Speculations about amplified feedbacks or catastrophic impacts, which are strongly disputed within the scientific community, are also taken into account, whereas potential negative feedbacks are ignored. Therefore, a value of $5.5^{\circ} \mathrm{C}$ for $\Delta \mathrm{T}_{2 \times \mathrm{CO} 2}$ is adopted, this being one of the highest estimates to be found in the literature (Dickinson, 1986).

The egalitarian myth of an ephemeral natural system results in high cost estimates of the impacts on the human system. The egalitarians are assumed to believe in a highly nonlinear damage curve, resulting in a $32 \%$ loss of economic output, if temperature increased by $3^{\circ} \mathrm{C}$ in the course of the next century, these values being based on the high range estimates offered by Nordhaus (1994).

The increasing level of technological development is not stimulated by egalitarians, insofar as it leads to greater pressure on the environmental system. Therefore, a low rate of technological development $\left(\delta_{\mathrm{s}}\right)$ is assumed. Technology enabling decarbonization of the economy is stimulated by the egalitarians and they are optimistic about the rate of improvement. Although cost considerations are not important to egalitarians, a low mitigation cost per unit of reduction is assumed, which reflects their optimism about the efficacy of clean technology.

The assumptions associated with the various perspectives which affect a number of crucial parameters within the model can be found in Table 8.1. 
Table 8.1: Parameter values in which the perspectives differ in their world view.

\begin{tabular}{|l|c|c|c|}
\hline & Individualist & Hierarchist & Egalitarian \\
\hline Climate sensitivity & low & best-guess & high \\
$\Delta \mathrm{T}_{2 \mathrm{icoz}}$ & 0.5 & 2.5 & 5.5 \\
Damage costs & low & moderate & high \\
$\theta_{1}$ & 0 & 0.0014 & 0.004 \\
$\theta_{2}$ & 0 & 2 & 4 \\
Technological development & high & moderate & low \\
$\delta_{2}$ & 0.004 & 0.012 & 0.002 \\
Mitigation costs & & & \\
$\mathrm{b}_{1}$ & high & moderate & low \\
$\mathrm{b}_{2}$ & 0.25 & 0.11 & 0.05 \\
$\delta$ & 3.5 & 2.9 & 2.3 \\
\hline
\end{tabular}

\subsubsection{Management Style}

The so-called management styles are modelled as a set of simple decision rules. During the simulation period, the preferred management style of each perspective is determined every year. The individual agents, however, adhere to management styles which lie within the perspective triangle (Figure 8.2). The average perspective adhered to by the agents determines the implemented policy ( $\mathrm{I}$ and R), which is the (weighted) average of the preferred policies of the three active perspectives. Thus: $I=\frac{1}{N} \sum_{i=1}^{N} a_{i}^{E} \cdot I^{E}+\frac{1}{N} \sum_{i=1}^{N} a_{i}^{I} \cdot I^{i}+\frac{1}{N} \sum_{i=1}^{N} a_{i}^{H} \cdot I^{H}$

$$
R=\frac{1}{N} \sum_{i=1}^{N} a_{i}^{E} \cdot R^{E}+\frac{1}{N} \sum_{i=1}^{N} a_{i}^{I} \cdot R^{I}+\frac{1}{N} \sum_{i=1}^{N} a_{i}^{H} \cdot R^{H}
$$

The agents are assumed to lack perfect knowledge of the system both in the present and in the future. Decisions are made on the basis of the agents' expectations of the future, their beliefs, wants and needs, and their observations of the system. The: assumptions which are made in framing decision rules are mainly based on Schwartz and Thompson (1990), Thompson et al. (1990) and Rayner (1991). 
MeEting TARgets

\section{Individualists}

Consistent with their characteristics, individualists prefer an adaptive management style. While they assume that a new equilibrium in the natural system will provide new opportunities for smart individualists, no active climate policy is advocated. In the event of the occurrence of negative effects on economic development, technological innovation will cancel out these negative impacts of climate change. In line with their ambition for economic growth, at least a minimum level of economic growth is desired. A simple adaptive strategy is assumed. so that in the event of realized economic growth falling below a certain minimum level, investments in economic development will be increased. Thus:

$$
\begin{aligned}
& \text { if } d Y(t)<\min [d Y] \text { then } I^{\prime}(t)=\min \left(1.0, \min [d Y] \cdot \frac{I(t-1)}{d Y(t-1)}\right) \\
& \text { else } I(t)=I(t-1)
\end{aligned}
$$

where

$$
d Y(t)=\frac{Y(t)-Y(t-I)}{Y(t-l)}
$$

where min[dY] is the minimum economic growth preferred by the individualist. The value of the minimum growth rate is determined by experiments on the utopian world for the individualist (see Section 8.5.2). Assuming that, in such a world, a collapse in consumption per capita is not desired, my experiments arrive at a minimum growth rate of $2 \%$.

With regard to emission reduction, the individualist will similarly advocate an adaptive strategy. Although individualists do not believe in economic risks due to climate change, if damage costs exceed a certain threshold value, of, for example, $1 \%$ of economic output, fossil fuel transition is assumed to be accelerated, while assuming a minimum half-life time of 20 years. This approach is somewhat similar to that adopted by Lempert et al. (1996). Thus:

$$
R^{l}(t)=20+\left(R^{\prime}(t-1)-20\right) \cdot 0.99
$$

If no significant economic damage is detected, the half-life time of a fossil transition is assumed to be the longer period of 1000 years. 


\section{Hierarchists}

Consistent with the general characteristics of hierarchists, their preferred management style is one of control. It envisages a balance between the levels of anthropogenic pressures on the climate system and the level of structural changes in the economic system, which are tolerated to meet environmental targets. Such a costbenefit approach is adopted in the simple rules for the hierarchistic management style.

To meet the needs of the society, a stable economic growth is desirable D[dY]. The preferred level of investments is, therefore, the one which leads to an expected growth of $\mathrm{D}[\mathrm{dY}]$. While abrupt changes in the investment levels need to be avoided, the preferred level of investments is a function of the level of investments of the previous year, to some extent corrected to meet the desire for a stable growth path of the economy, thus:

$$
I^{\prime \prime}(t)=0.9 \cdot I(t-I)+0.1 \cdot \frac{D[d Y]}{d Y(t-l)} \cdot I(t-I)
$$

Experiments with the utopia case for the hierarchist (see Section 8.5.2) yield a figure for desired growth of $1.5 \%$ a year. A higher growth rate is not desirable, since consumption per capita collapses in the event of higher desirable growth rates, which are avoided in a utopian world.

Hierarchists prefer to avoid acting under extreme uncertainty. Therefore; measures to reduce emissions are embarked upon when temperature change is far. from dangerous levels. I assume an upper level of a temperature increase of $2{ }^{\circ} \mathrm{C}$ relative to 1900, a figure based on the UNEP's Advisory Group on Greenhouse Gases (AGGG, 1990). Given the temperature increase of about $0.5^{\circ} \mathrm{C}$ over the past century, the maximum temperature increase for the next century would be $1.5^{\circ} \mathrm{C}$. If measured temperature is below the threshold value of $0.5^{\circ} \mathrm{C}, \mathrm{R}$ is assumed to be equal to 100 years. Emission reduction measures are slowly implemented. when the measured. temperature increase $\mathrm{M}[\Delta \mathrm{T}]$ exceeds the $0.5^{\circ} \mathrm{C}$ increment. The implementation of measures is reinforced once the $1.0{ }^{\circ} \mathrm{C}$ level and the $1.5^{\circ} \mathrm{C}$ level are violated. Furthermore, $\mathrm{R}$ is assumed to cannot fall below a period of 20 years.

The levels of changes as listed below have been determined by experiments in the utopian case whereby the maximum increase is $1.5^{\circ} \mathrm{C}$ (Section 8.5.2). 
MEETING TARGETS

$$
\begin{gathered}
\text { if } M[\Delta T(t)]<0.5 \text { then } R^{H}(t)=100 \\
\text { if }(M[\Delta T(t)]>0.5) \wedge(M[\Delta T(t)]<1.0) \\
\text { then } R^{\prime \prime}(t)=20+\left(R^{H}(t-1)-20\right) \cdot 0.995 \\
\text { if }(M[\Delta T(t)]>1.0) \wedge(M[\Delta T(t)]<1.5) \\
\text { then } R^{H}(t)=20+\left(R^{H}(t-1)-20\right) \cdot 0.99 \\
\text { if } M[\Delta T(t)]>1.5
\end{gathered}
$$$$
\text { then } R^{\prime \prime}(t)=20+\left(R^{\prime \prime}(t-1)-20\right) \cdot 0.98
$$

\section{Egalitarians}

Egalitarians prefer a preventive management style. In order to guard the society against a full-blown catastrophe in the long term, drastic structural social, cultural and institutional changes are necessary, notwithstanding any short-term disadvantages and costs which may result.

Insofar as climate policy is concerned, a rapid transition towards a fossil free society is the ultimate goal of the egalitarian, which implies the choice of a low halflife time of 20 years $\left(R^{\mathrm{E}}=20\right)$. The level of investments is determined by the assumption that no more investments are made than those required to compensate for the depreciation of existing capital goods $(\mathrm{dK} / \mathrm{dt}=0$ in equation 8.4$)$ :

$$
I^{E}(t)=\frac{\delta_{k} \cdot K(t-1)}{Y(t-1)}
$$

Economic output $(\mathrm{Y})$ will only grow as a result of an increase in the labor force and will occur under a low level of technological development.

\subsubsection{Evaluating Perspectives}

According to Thompson ef al. (1990), people are assumed to abandon their perspectives in the event of surprise, if observations differ from expectations. Perspectives are assumed to shift towards, world-views which can better explain the observed behavior of the system. Therefore, this learning and adaptive behavior of agents is simulated as a "Battle of Perspectives" using a genetic algorithm. Defining a 212 
fitness function for measuring the fitness of an agents' perspective is almost an impossible task. The fitness function is assumed to be required to measure the likelihood of the perspectives being adhered to. This likelihood may be expressed in terms of the difference between the measured values in the real world and the expected value associated with the view of the agent. In the simple world as constructed in this Chapter, the agents' perspective only deals with temperature change. The fitness function is, therefore, a function of expected temperature change due to measured $\mathrm{CO}_{2}$ concentration and the measured value of temperature change for a historical period. A simple model is used to quantify the expectation of perspective $i$ as given below:

$$
E^{\prime}(\Delta T)(t)=\frac{\Delta T_{2 \mathrm{acos}}}{\ln (2)} \cdot \ln \left(\frac{\mathrm{M}[\mathrm{pCO}](t)}{p C O 2_{\text {in }}}\right)
$$

where $\Delta \mathrm{T}_{2 \mathrm{x} \times \mathrm{2} 2}$ is the climate sensitivity according to the perspective of the agent and $\mathrm{M}\left[\mathrm{pCO}_{2}\right]$ is the measured concentration of $\mathrm{CO}_{2}$. Although I recognize the shortcomings of this approach, in order to render an initial implementation as clear as possible, I assume that one of the "extreme" agents has a perfect picture of the system.

Usually, the measured temperature change tends to be equal to the observed global mean temperature change. If the difference between the measured and the expected value is less than a tolerance level, the fitness of the agents' perspective is assumed to be maximal; i.e. the agents concerned have no reason to alter their perspective. This tolerance level is included to take account of the ignorance of agents. Although expectations may differ from measurements, ignorance prevents agents from changing their perspectives. Therefore, ignorance of surprises may delay the learning and adaptive behavior of the agents.

In mathematical terms, this enables me to write the following equations; in the event of the difference between expected and measured levels being greater than a tolerance level, the agents may be surprised and be moved to alter their perspective. The resulting fitness function is shown in Figure 8.3.

In the event of the measured value $\mathrm{M}(\Delta \mathrm{T})$ being smaller than the expected value minus the tolerance level $\left(\mathrm{E}_{l}(\Delta \mathrm{T})\right.$-tol), the fitness function for agent $\mathrm{i}$ is defined as:

$$
f_{i}=\exp \left(-\left(E_{i}(\Delta T)-M(\Delta T)-t o l\right)^{2}\right)
$$


while in the event of $M(\Delta T)$ being greater than $E_{i}(\Delta T)+$ tol, the fitness function is defined as:

$$
f_{i}=\exp \left(-\left(M(\Delta T)-E_{i}(\Delta T)-t o l\right)^{2}\right)
$$

The genetic algorithm and the dynamic system are integrated in the following manner. Instead of scenario analysis whereby a model as described in Section 8.3 simulates the effects of assumptions made for the control rates $I$ and $R$, or an optimization model with which the optimal values of the control rates $I$ and $R$ are determined, for the purposes of my "Battle: of Perspectives", the control rates are a function of the state variables. The values $I$ and $R$ are the (weighted) averages of $I_{i}$ and $R_{i}$ the individually preferred values for the control variables. These preferences may change, in time, due to changes in the system. For example, if the dominant perspective of the agents concerned is individualistic at the start, it may change as a reaction to a persistent series of surprises into a more hierarchistic or egalitarian perspective, if the agents are confronted with serious impacts of climate change.

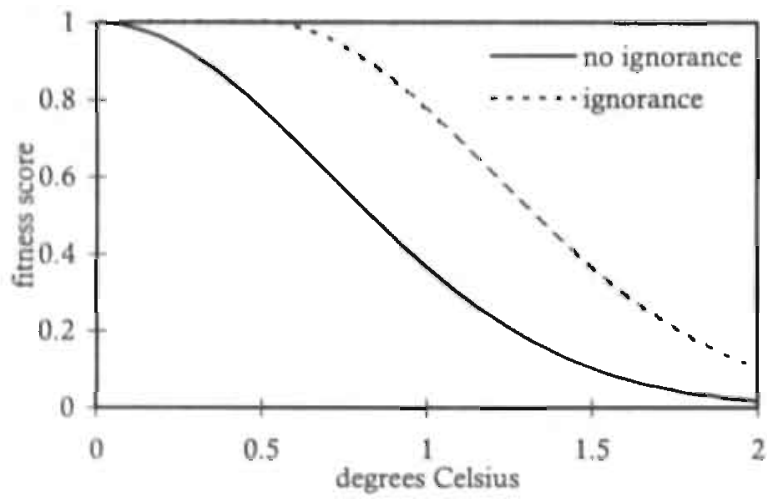

Figure 8.3:Fitness function of agents'perspective related to the mismatch in expectations and observations. For ignorance tol $=0.5$ is used and for no-ignorance tol $=0$ is used. 


\subsection{Exploratory Experiments}

\subsubsection{Introduction}

In this Section, the utopias and dystopias for the three perspectives are analyzed first. The utopias and dystopias are a static view of a single global commoner, but can be used to scan the space of possible futures. In the second part, a set of agents exists with perspectives located at various places within the triangle spanned up by the three active perspectives. Due to learning via observations of the system, the favored climate policy may change over time among the agents. This may lead to a set of possible images of the future. Finally, an attempt is made to analyze the influence of surprises on the change in perspectives.

\subsubsection{Utopias and Dystopias}

The concept of utopias is used to determine a number of variables in implementing the management styles (Section 8.4.3). Utopias and dystopias can be simulated by running the model for a case in which a global commoner is in charge, i.e. an agent who adopts a perspective which is located at a corner of the triangle of perspectives (Figure 8.4). Furthermore, the global commoner does not learn from the information one derives from the system. In the case of a utopia, the world fits very well with ones expectations, but in the case of a dystopia, there is a mismatch of expectations and measurements. In order to investigate the potential consequences of such a mismatch, I am obliged to assume that the global commoner does not learn and adapt.

\section{Utopias}

The utopias are used to fill in a number of parameters of the management styles as discussed in Section 8.4.3. The derived results, therefore, correspond to subjective ideas of perspective utopias:

In the egalitarian utopia (Figure 8.4), emissions of fossil $\mathrm{CO}_{2}$ are phased out within a few decades, leading to a modest temperature increase of $0.5^{\circ} \mathrm{C}$. Economic growth is approximately $1 \%$ a year.

In the hierarchistic utopia, the economy grows at a stable rate of $1.5 \%$ a year (Figure 8.4). Due to timely implementation of emission reductions, the temperature. increase stabilizes at around $1.5^{\circ} \mathrm{C}$ above present values which is assumed to be the upper boundary of acceptable temperature change. 
In the individualistic utopia, economic growth is greater than $2 \%$ a year, leading to an increase of fossil $\mathrm{CO}_{2}$ emissions to $40 \mathrm{GtC}$ in 2100 (Figure 8.4). However, due to the stability of the natural system, a human-induced climate change is rather modest, leading to a $0.5^{\circ} \mathrm{C}$ increase in 100 years. This temperature change has no significant impact on economic activities, so that the use of fossil fuels need not be restricted.

A more interesting situation is a difference in management style and world view:

\section{Dystopias}

In the event of the system functioning according to the egalitarian perspective (Figure 8.4), a hierarchic management style leads to a higher economic growth. Although fossil $\mathrm{CO}_{2}$ emissions are reduced, a temperature increase up to $2.5^{\circ} \mathrm{C}$ can not be avoided. An individualistic management style may lead to an absolute collapse in economic development, due to excessively high economic growth aspirations, together with severe impacts of climate change. The emission reduction measures are implemented at too late a juncture to avoid a temperature increase in excess of $4{ }^{\circ} \mathrm{C}$.

In a system which functions according to the view of the hierarchist (Figure 8.4), an egalitarian management style implies low economic growth. The reduction of $\mathrm{CO}_{2}$ emissions leads to a stabilization of the temperature increase. In the event of the individualistic management style being implemented, the economic output increases fast and fossil $\mathrm{CO}_{2}$ emissions increase to about $30 \mathrm{GtC}$. However, temperature increases to a level of $2.5^{\circ} \mathrm{C}$.

In a stable system functioning according to the world-view of the individualist (Figure 8.4), a phase-out of fossil fuels would be a waste of money, since this would suppress economic growth while, despite the absence of control, temperature change would not harm economic development. The management styles of the egalitarians and hierarchists are associated with low economic growth.

If the global commoner adopted an egalitarian management style, the temperature increase would be lower than expected in the event of the world functioning according to the hierarchic or individualistic world-view, and, thanks to accelerated technological progress, economic growth would be higher than expected.

A hierarchic management style, however, leads to exceeding the $1.5^{\circ} \mathrm{C}$ maximum increase in temperature, if the world functions according to the egalitarian world view. If the world functions according to the individualistic view, higher economic growth and a lower temperature increase will occur.

An individualistic management style may lead to lower economic growth, if the world functions according to the egalitarian or hierarchic world-view. In the egalitarian, world, the economy would be seriously damaged by climate change. 

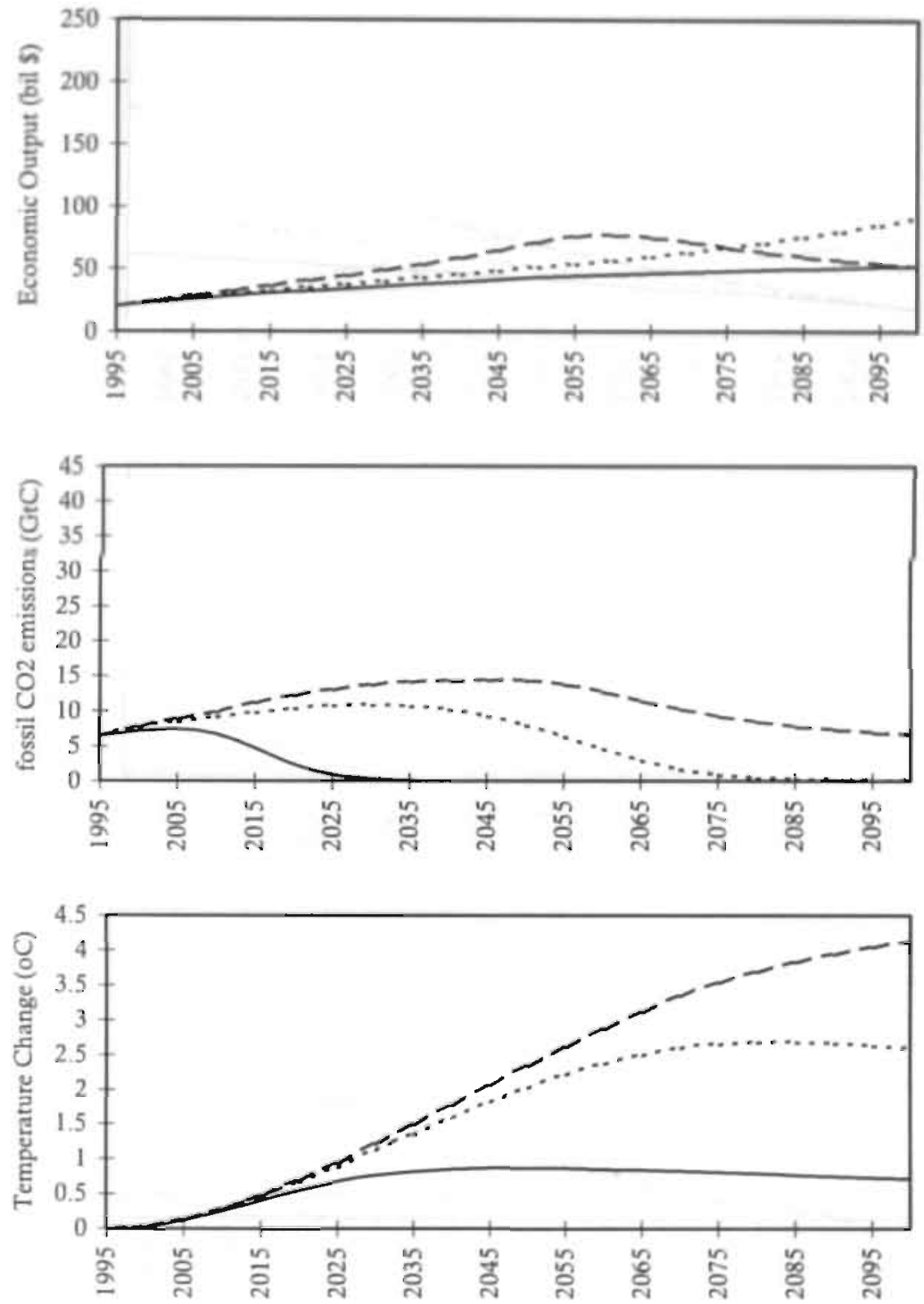

Figure 8.4a: Projections if the world functions according to the world view of the egalitarian and an egalitarian $(\zeta$ ), hierarchistic (..) or individualistic (-) management style is implemented, and the agents do not learn and adapt. 

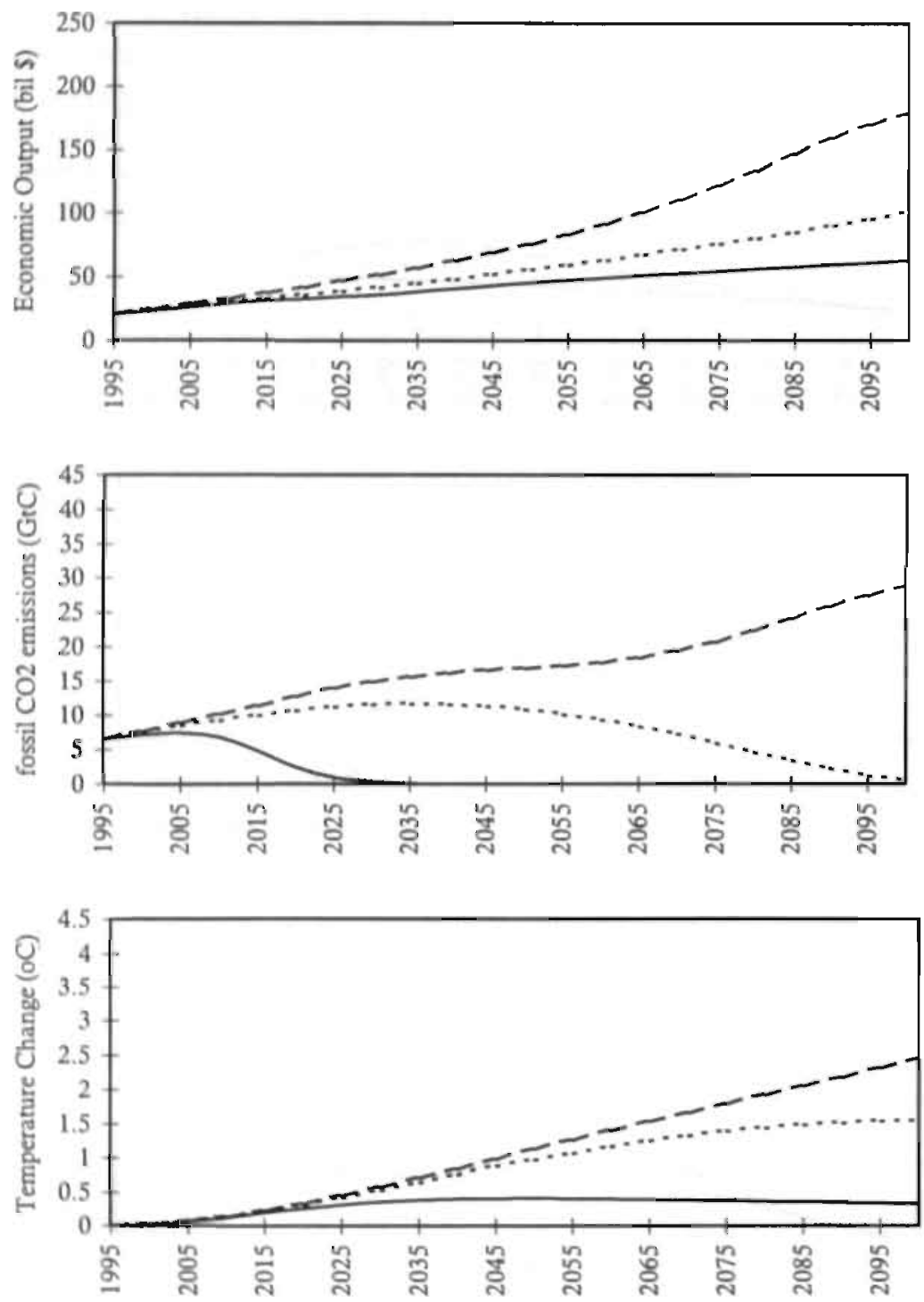

Figure 8.4b:Projections if the world functions according to the world view of the hierarchist and an egalitarian $(\circlearrowleft$ ), hierarchistic (...) or individualistic (-) management style is implemented, and the agents do not leam and adapt. 

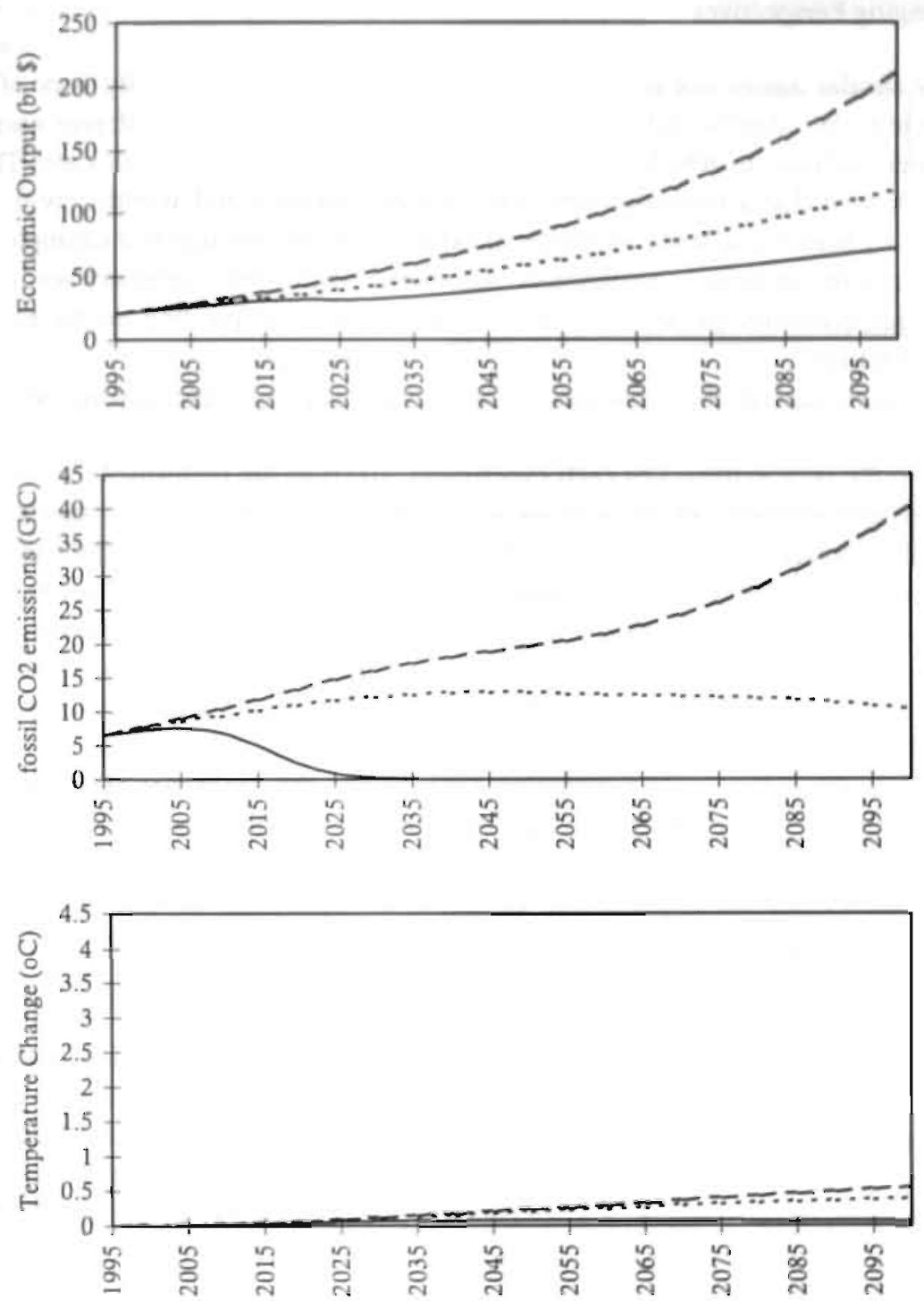

Figure 8.4c: Projections if the world functions according to the world view of the individualist and an

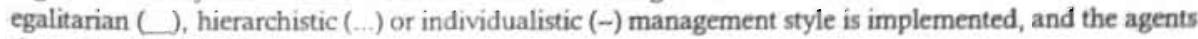
do not learn and adapt. 


\subsubsection{Changing Perspectives}

Fifty similar agents are included in the "Battle of Perspectives". By way of a sensitivity test, the adaptive behavior is analyzed in the case of three different initial mixes of perspectives, in which a different perspective dominates in each case. The dystopias showed that a mismatch between management style and world-view does not necessarily lead to collapses. However, if expectations are not met (for example, a high temperature increase), preferences associated with other perspectives (for example, high economic growth) will secure a more dominant position, in the event of agents adapting.

In order to analyze the consequences of the various perspectives among agents, the experiments are performed for three sets of assumptions of the global system according to the perspectives. For each experiment, 100 runs are performed for which the average and extremes of the various indicators are determined. For analytical purposes, the average values are mainly depicted. The starting situation is comparable with the frame of utopias and dystopias, as discussed in the previous section: a dominating management style $\mathrm{X}$ in a world which functions according to perspective $\mathrm{Y}$. However, the agents' perspectives may now change in time, and none of the perspectives fully determine the climate change policy (Figures 8.5 and 6). A. tolerance level of $0.5^{\circ} \mathrm{C}$ is used as a measure for ignorance.

In the event of the world functioning according to the egalitarian world view, economic output grows faster in case of initially dominating hierarchic or individualistic management styles, but levels off due to a high temperature increase. Emissions reductions are implemented earlier, if an egalitarian management style dominates at the start, resulting in a temperature stabilizing below $2^{\circ} \mathrm{C}$; otherwise an increase of more than $2^{\circ} \mathrm{C}$ is expected. In a world which functions according to the hierarchist, the temperature target of $1.5^{\circ} \mathrm{C}$ will just not be met, irrespective of the initial management style. Emissions stabilize at doubled the present level. If an egalitarian management style dominates at the start, the levelling off of emissions would only be temporary, in a world which functions according to the individualistic world view. The revival of the individualistic management style leads to an increase in emissions which, in turn, results in a temperature just below $0.5^{\circ} \mathrm{C}$ by the year 2100 . Starting with a hierarchic or individualistic management style, both lead to a high level of economic development.

In the exploratory experiments, agents seem to be able to adapt to changing insights in the functioning of the global system. This is not surprising, because the agents are modelled accordingly. In Figure 8.6, the transition of perspectives is visualized. It shows that the perspectives of agents converge to the "real" functioning 220 
of the system, although this might occur in very different paths with different levels of success.
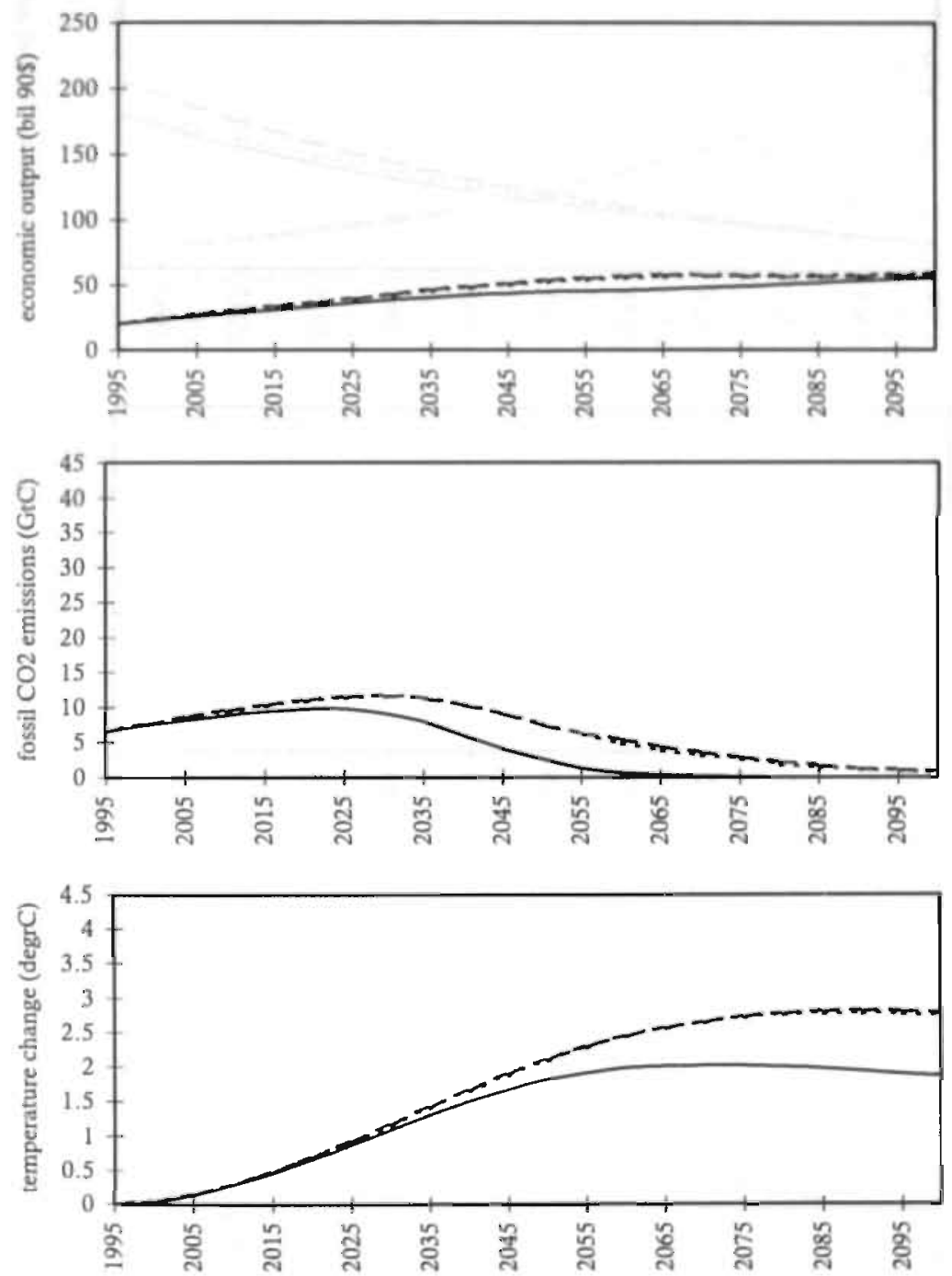

Figure 8.5a: Average projections if the world functions according to the world view of the egalitarian and an egalitarian $($, hierarchistic (...) or individualistic (-) management style is implemented, and the agents do learn and adapt. 

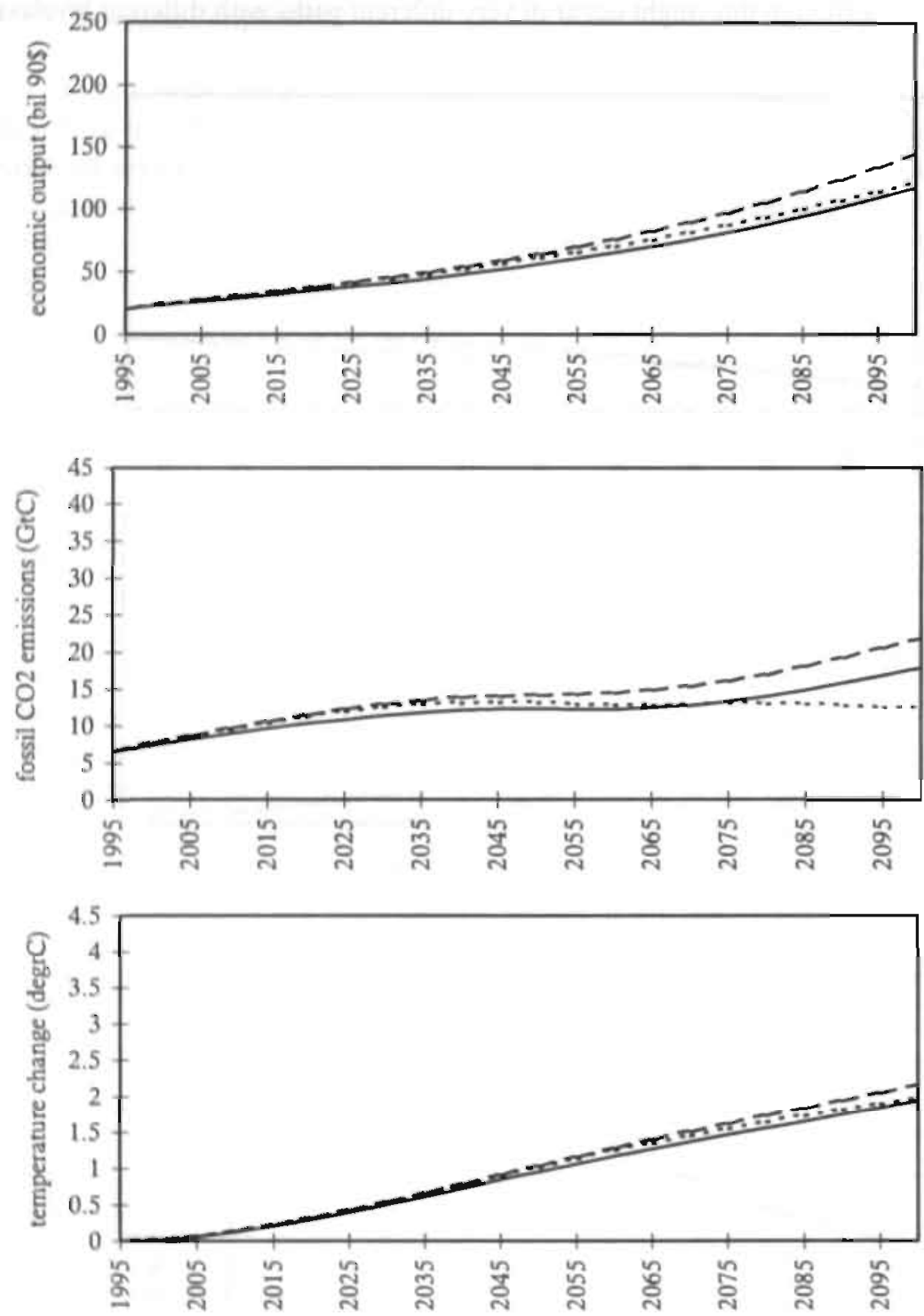

Figure 8.5b: Average projections if the world functions according to the world view of the hierarchist and an egalitarian $(\zeta$, hierarchistic (...) or individualistic (-) management style is implemented, and the agents do leam and adapt. 

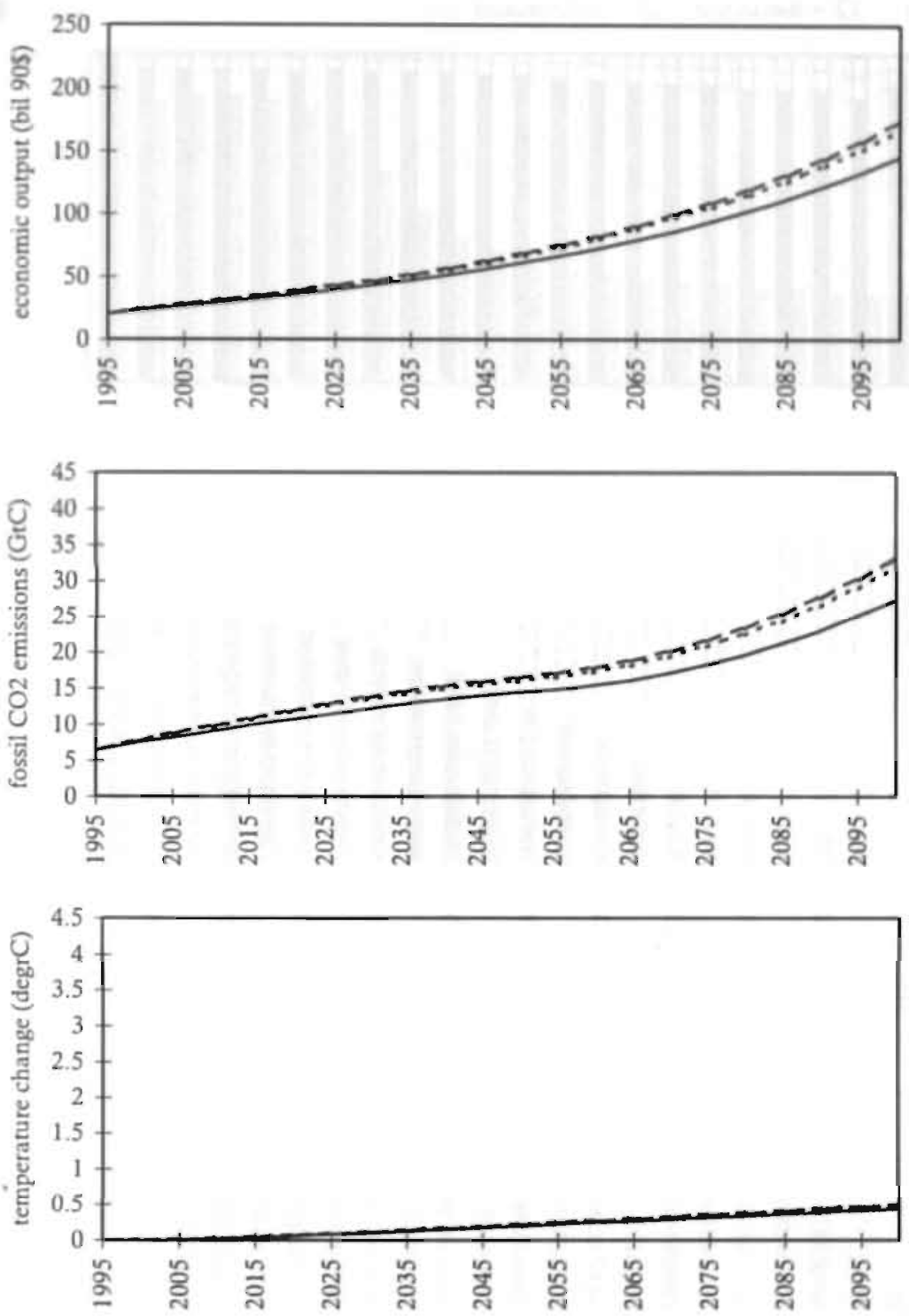

Figure $8.5 \mathrm{c}$ : Average projections if the world functions according to the world view of the individualist and an egalitarian ( ), hierarchistic (...) or individualistic (-) management style is implemented, and the agents do learn and adapt. 
MEETING TARGETS
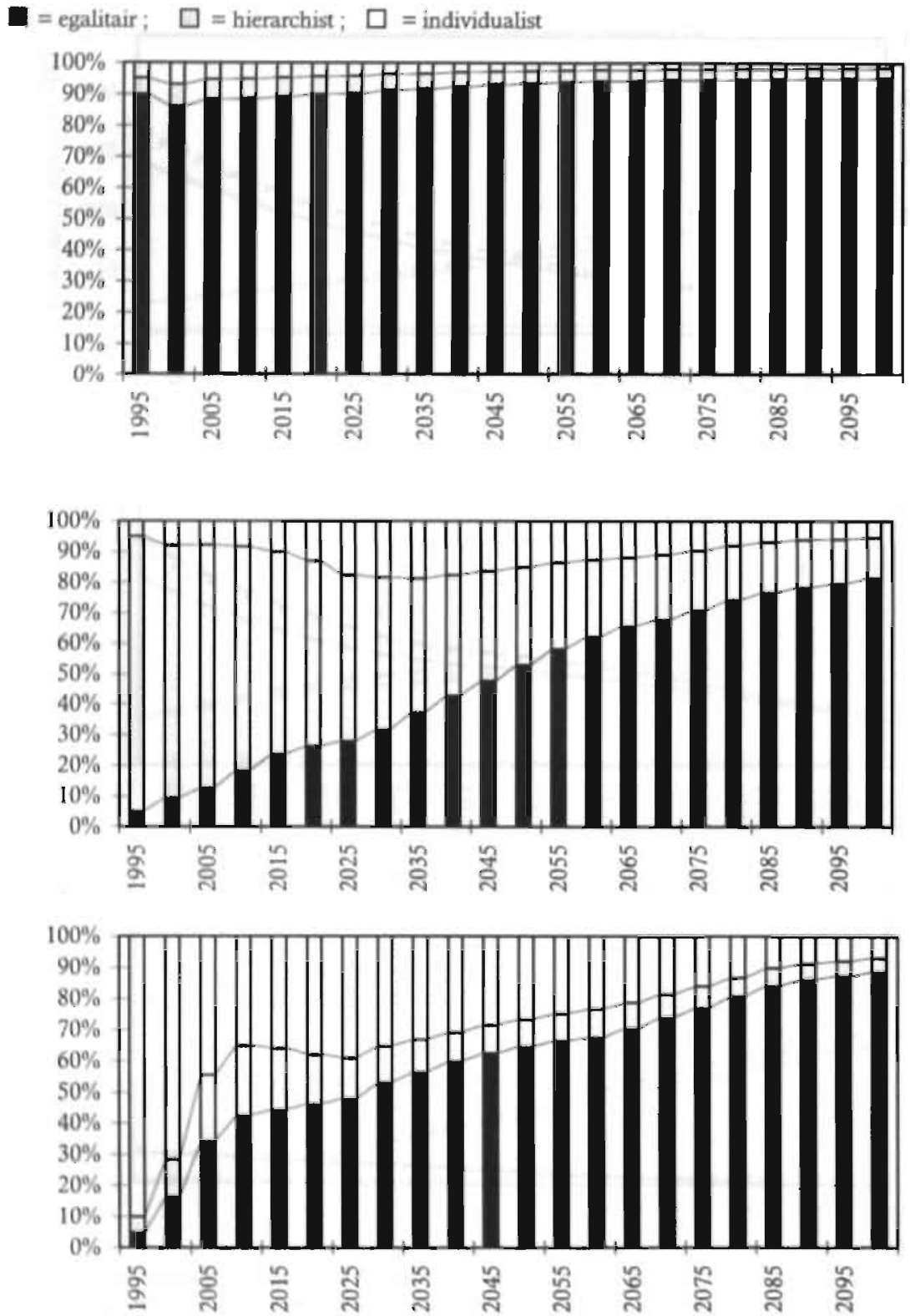

Figure 8.6a: Perspective changes if the world functions according to the world view of the egalitarian and the initial management style is egalitanian (upper), hierarchistic (middle) or individualistic (lower). 

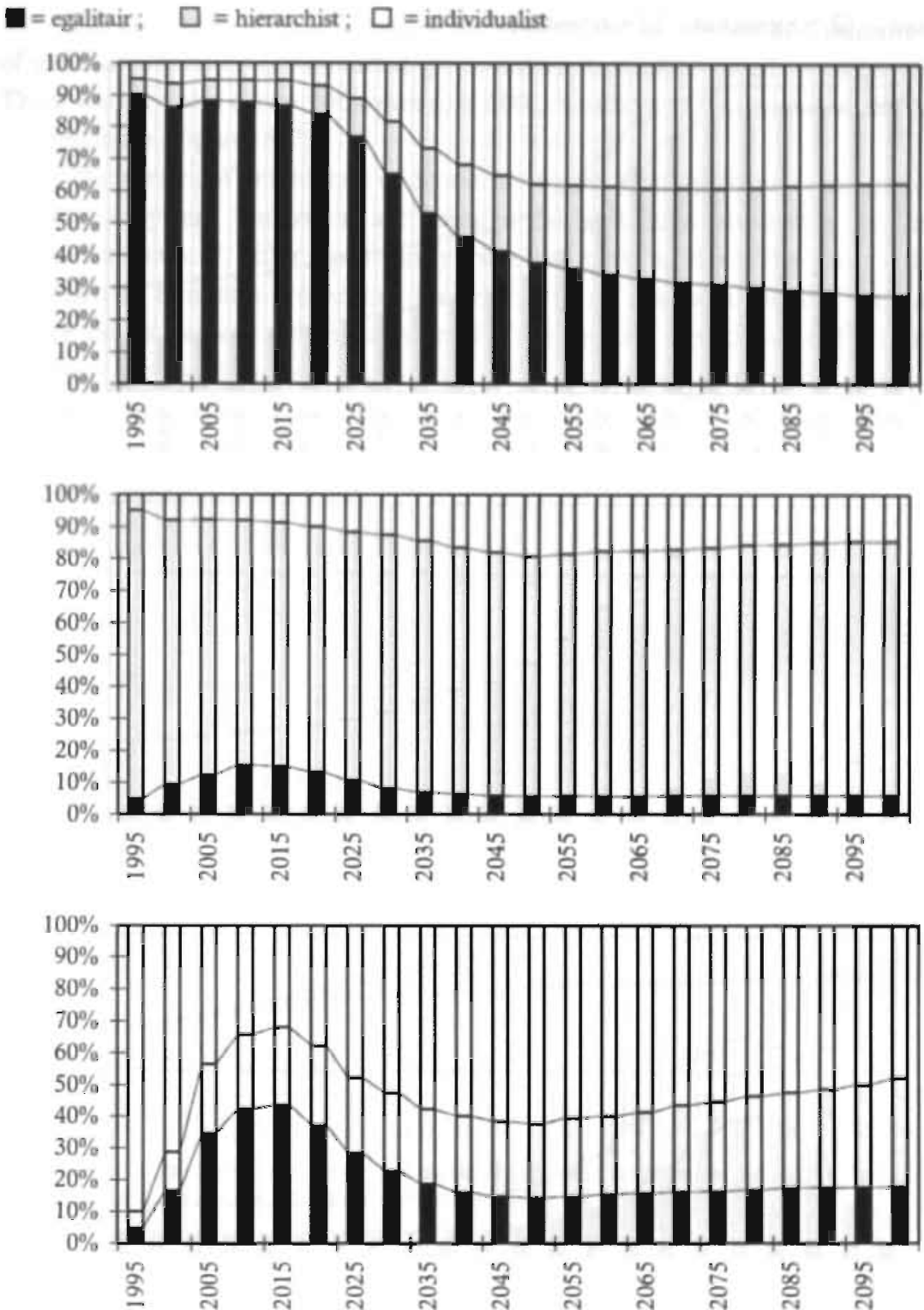

Figure 8.6b: Perspective changes if the world functions according to the world view of the hierarchist and the initial management style is egalitarian (upper), hierarchistic (middle) or individualistic (lower). 
$\mathbf{\square}$ = egalitair $\quad \square$ = hierarchist $; \quad \square=$ individualist
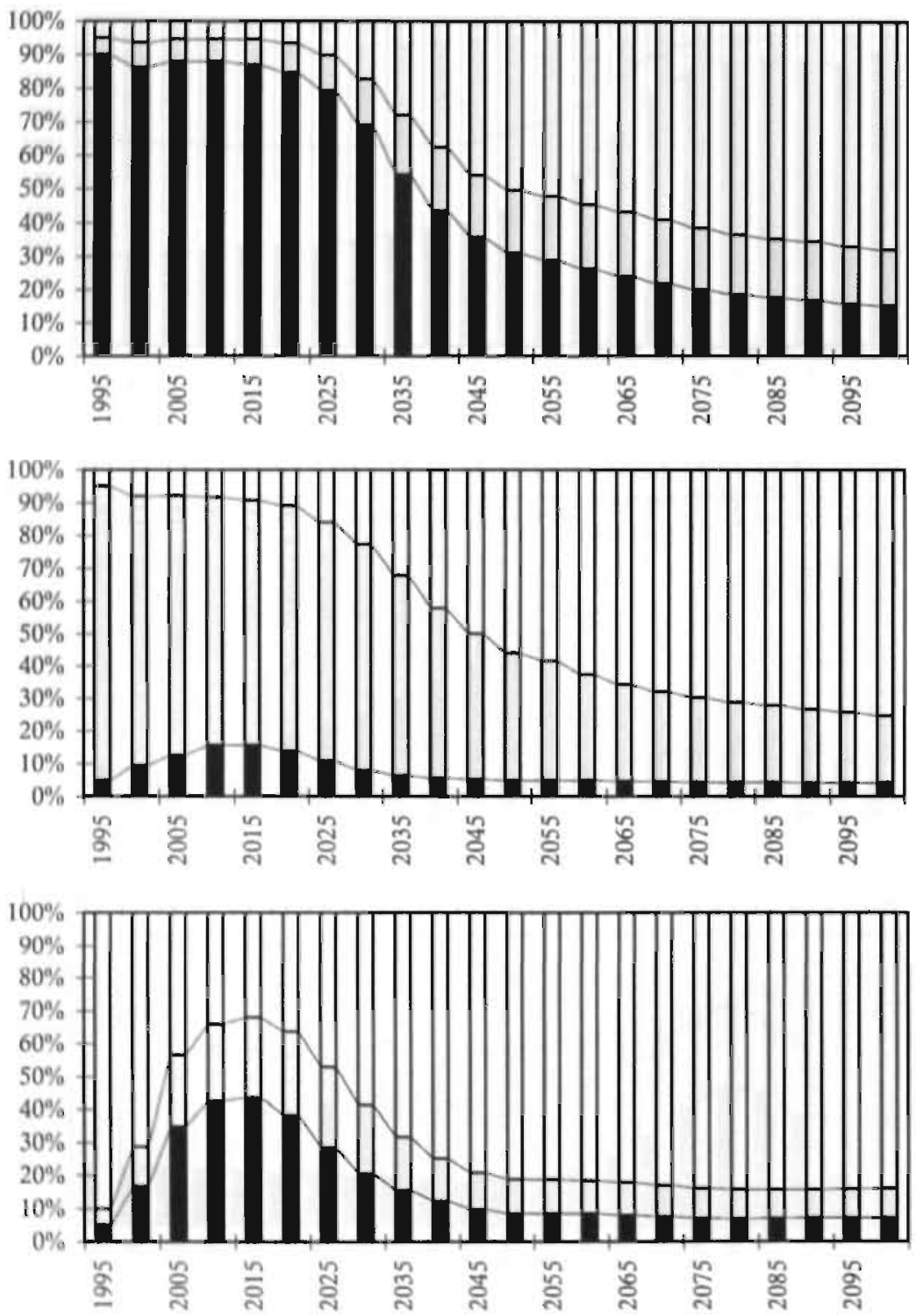

Figure 8.6c: Perspective changes if the world functions according to the world view of the individualist and the initial management style is egalitanian (upper), hierarchistic (middle) or individualistic (lower). 
For each type of functioning of the system, the maximum and minimum values of various indicators are recorded given different possible initial management styles. Thus, each system is run 300 times $(3 \times 100)$, resulting in the spread of the indicators as depicted in Figures 8.7 .

The ranges of projections of economic output overlap largely during the coming decades. A system functioning according to the egalitarian world-view will, at the end of the next century, differ significantly from the other "worlds". The same difference is identified in emission projections for which, in an egalitarian world, emissions will reduce below present values at the end. Temperature projections differ significantly between the world-views. Egalitarian and hierarchic projections overlap when emissions are reduced successfully in the egalitarian world. The individualistic temperature projections will remain significantly below other projections, in spite of their high emission projections.

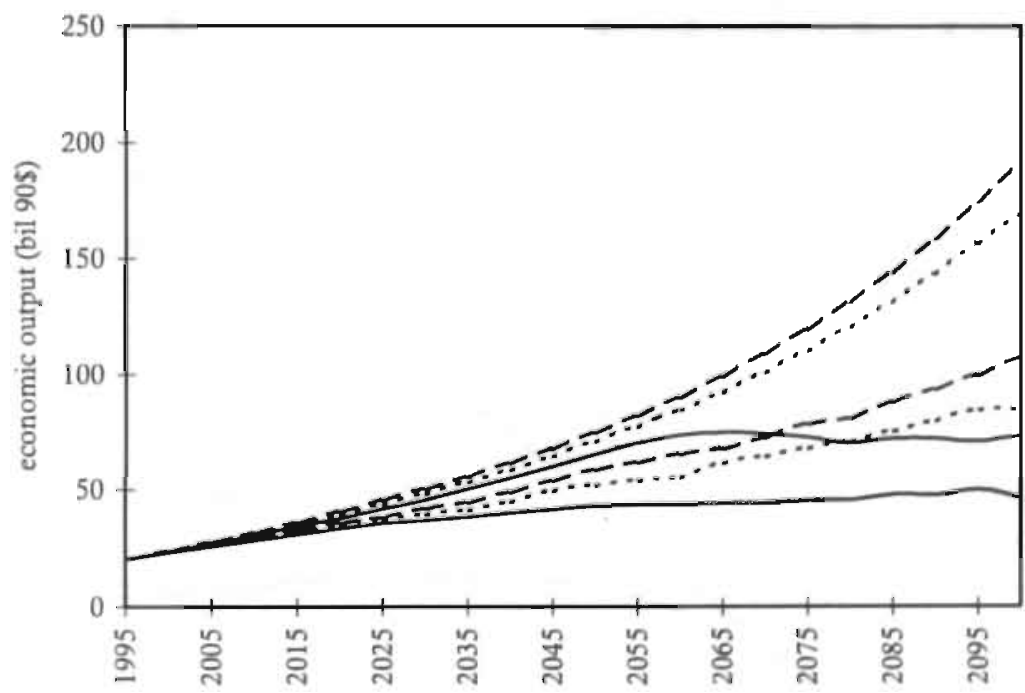

Figure 8.7a: Range of projected economic output if the world functions according to the world view of the egalitarian ( $)$, hierarchist (...) and individualist (-). 


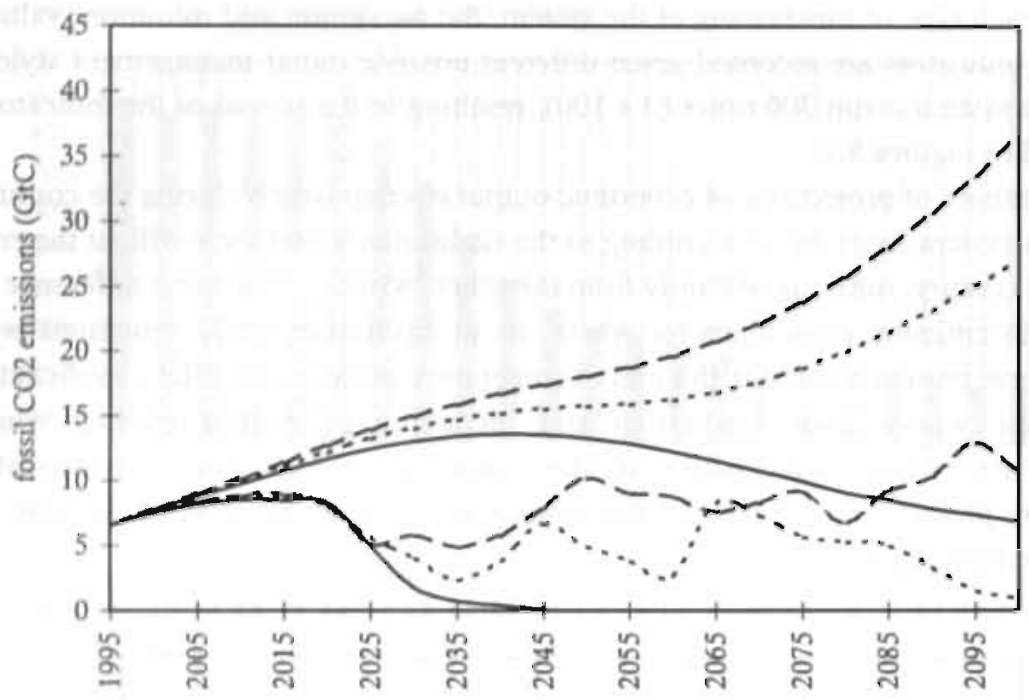

Figure 8.7b: Range of projected $\mathrm{CO}_{2}$ emissions if the world functions according to the world view of the egalitarian ( $($ ), hierarchist (...) and individualist (-)

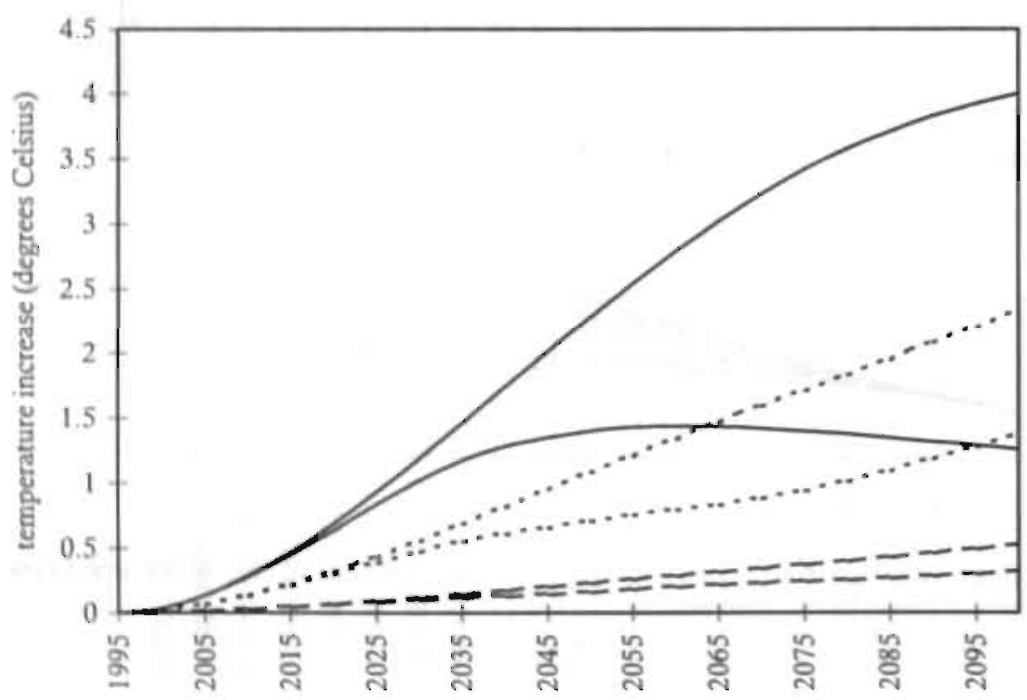

Figure $8.7 \mathrm{c}$ : Range of projected temperature change if the world functions according to the world view of the egalitarian $(\zeta$ ), hierarchist (...) and individualist (-). 
Can I conclude from this analysis that present policy does not matter in the long run, while learning and adaptive behavior will correct mistakes? I will not support this. kind of conclusion for two reasons: (1) the present model is extremely simple and does not represent the complex economic and climate dynamics in all aspects; (2) although agents learn from surprises, the framework is constructed in such way that the real picture of the system is within the triangle of perspectives. Within the model, reality can be known. However, insights change in time, and new insights emerge from the interaction between agents and the system. Therefore, there is a need for a changing triangle of perspectives, in which the corners, representing the extreme perspectives, change in time, but continue to explain historical facts. This moving triangle is a step too far for this moment, but an experiment will be analyzed in which agents are confronted with a surprise.

\subsubsection{Surprises}

Imagine a world, in which serious global warming may occur, due to human intervention in the global system. Suppose, furthermore, that this is a world in which the egalitarian perspective dominates at the start and agents do not ignore differences between observations and measurements. Suppose that observed global mean temperature is masked by an additional cooling effect, and this mask falls off in the middle of the next century. Then various agents are confronted with a surprise ${ }^{2}$. The cooling surprise may be explained in two ways: (I) over the next 50 years, global warming is masked by a cooling effect which disappears after 2050. (ii) over the next 50 years global warming is masked by a cooling effect. One discovers the causes and the cooling effect is discounted in the expectations.

In such a world, a cooling surprise would lead to a slowing-down of emission reduction, due to greater dominance of the hierarchic and individualistic perspectives (Figure $8.8 \mathrm{a}$ ). This results in an additional increase of about $1^{\circ} \mathrm{C}$ by 2100 , although this increase is not recognized before the middle of the next century. In Figure $8.8 \mathrm{c}+\mathrm{d}$, the perspective changes are depicted, which show that, in case of a surprise, the hierarchic and individualistic perspective become dominant. Although the share of the egalitarian perspective increases after the cooling effect was discovered, it is too slow to reduce the consequences of the delay. This experiment illustrates that a delayed response not only leads to a later reduction of emissions, but also that due to a lock-in of an individualistic management style, the emission reductions are implemented more slowly, leading to an extra delayed effect.

\footnotetext{
${ }^{2}$ Such a cooling surprise is not only a theoretical probability. The recent discovery of the global mean cooling effect of sulphate aerosols served also as a surprise for the main part of the climate change community.
} 


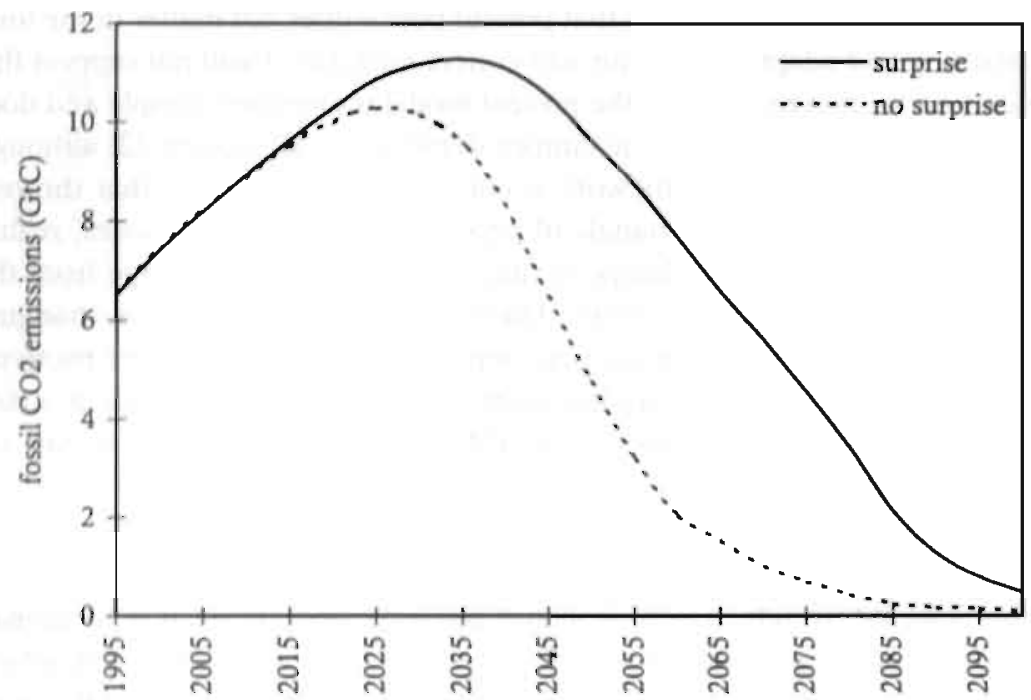

Figure 8.8a: Average projections of fossil $\mathrm{CO}_{2}$ emissions if the agents are confronted with a (cooling) surprise or not.

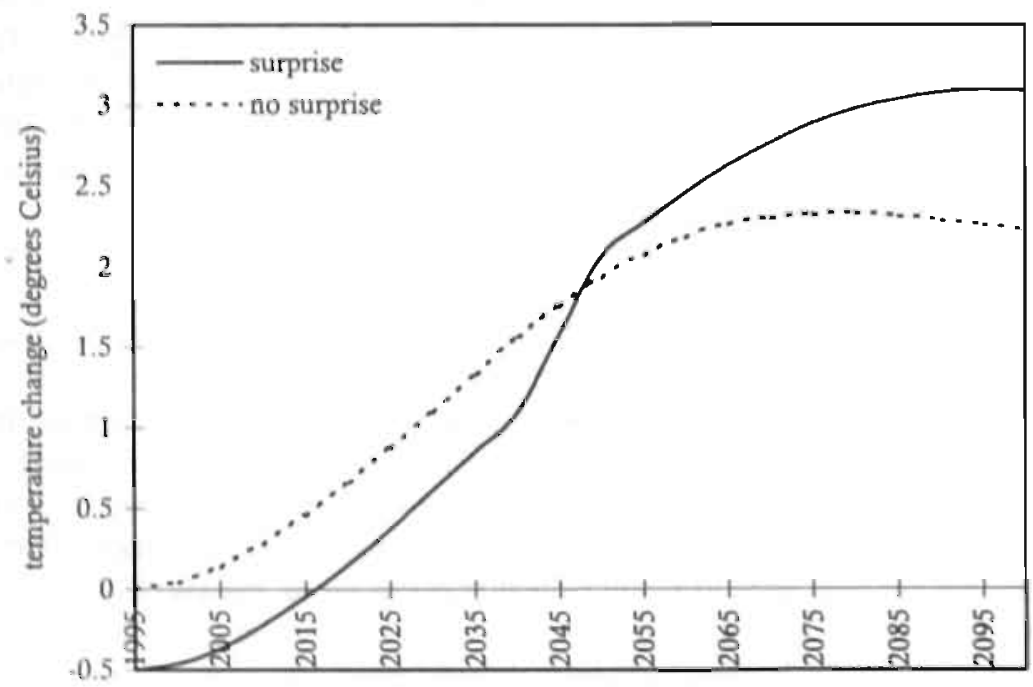

Figure $8.8 \mathrm{~b}$ : Average projections of temperature change if the agents are confronted with a (cooling) surprise of not. 
- = egalitair ; $\quad \square=$ hierarchis $; \quad \square=$ individualist

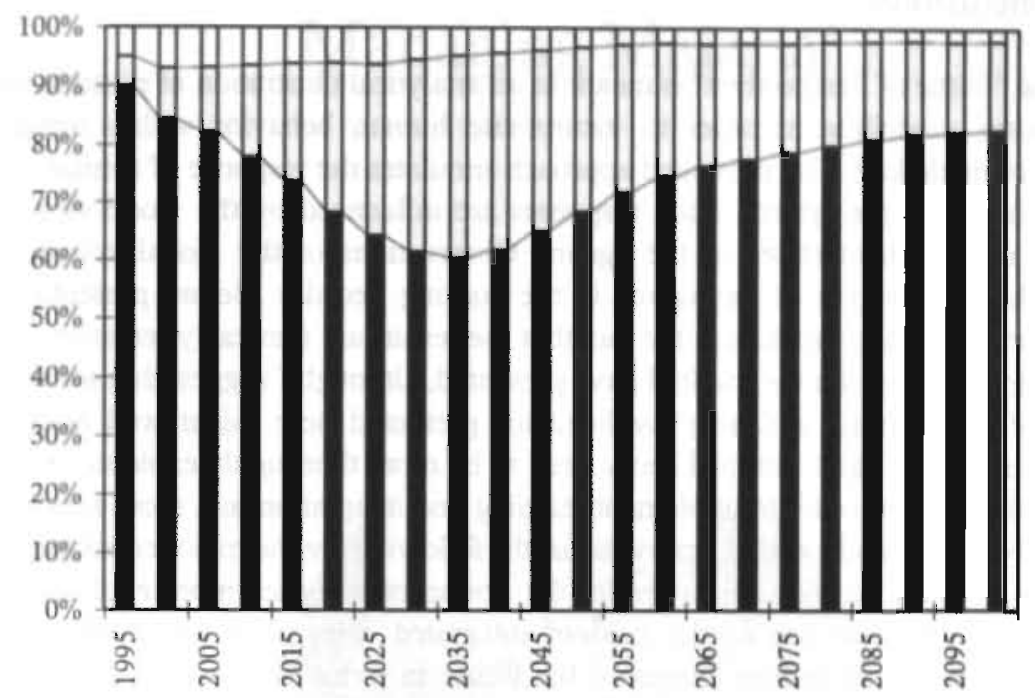

Figure 8.8c: Perspective change in case agents are not confronted with a surprise.

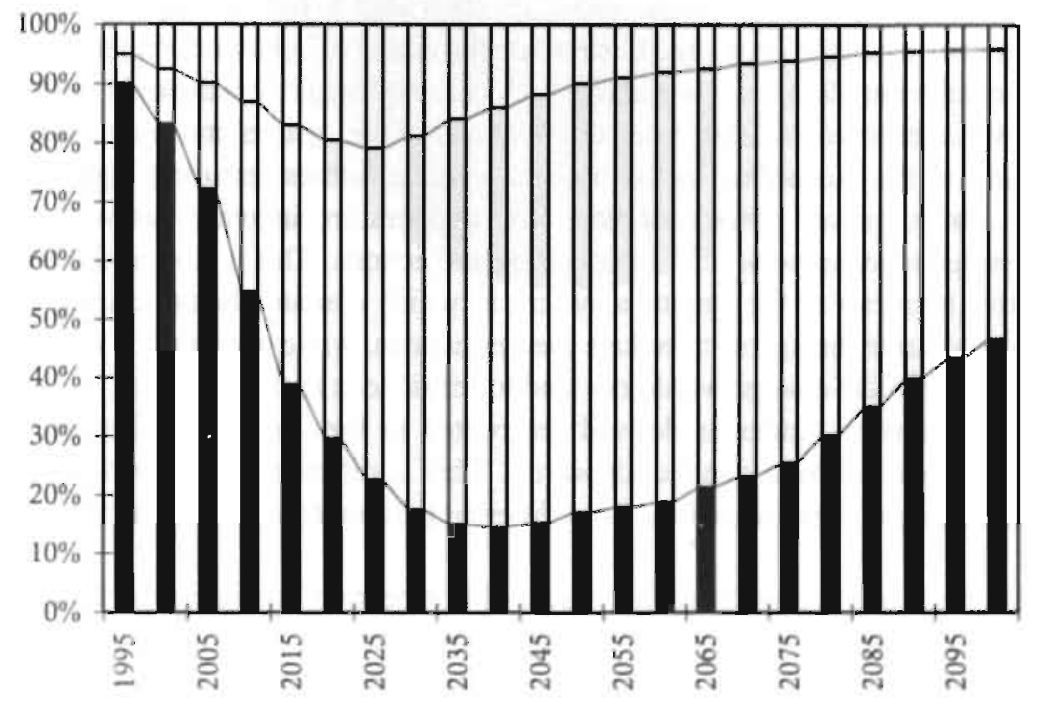

Figure $8.8 \mathrm{~d}$ : Perspective change in case agents are confronted with a surprise. 


\subsection{Conclusions}

The "Battle of Perspectives" is meant as an analytical illustration of possible use of evolutionary modelling in order to incorporate human behavior within integrated assessment modelling. The presented approach simulates the response of similar agents to the changes in the system. Such responses are influenced by the world-views and resulting management styles of the agents. Observations of the global system may change the perspectives of the agents in the coming decades. Before presenting the conclusions, I need to emphasize the fact that the results are inevitably tentative. There is no claim of realism for the model I have presented, although I suggest that some basic aspects of the learning and adaptive behavior presented here might well hold. The dynamic aspects of the Cultural Theory need to be more thoroughly explored than has yet been the case, in order to implement leaming and adaptation in a more satisfactory manner. Notwithstanding these reservations, the following are the major conclusions.

The concept enables me to render the notion of surprises more explicitly than earlier modelling activities in the field of integrated assessment for global climate change. Instead of projecting images of the future in terms of assumed or "optimal" policies, my approach tries to simulate the adaptive and learning, behavior of agents. The inclusion of various perspectives among agents is used to handle surprises explicitly, whereby genetic algorithms are used to simulate the adaptive behavior of agents.

The results show that, in a world in which climate change was the only problem to worry about, agents would learn from observations of the system and may adapt their management styles. According to the models, various futures featuring high levels of fossil $\mathrm{CO}_{2}$ emissions are associated with low temperature increases as well as low emission paths, together with high temperature projections. This is due to the learning and adaptive behavior of the agents: a system in which climate change caused serious impacts would drive the agents to reduce their emissions, while a system in which there was no serious climate change would not lead to restrictions in the long-run.

The uncertainty ranges as derived are rooted in the unpredictability of agents' behavior as modelled by a genetic algorithm. This is different from previous studies where uncertainty ranges are derived from different parameter-values for a deterministic model.

To summarize, the results of the "Battle of Perspectives" demonstrate the potency of a different concept in scanning the future. Taking account of the notion of learning and adaptation may lead us to new kinds of images based on the assumptions of the global system and the decision-rules adopted by agents. This approach may prove to enhance the insights into possible images of the future. 


\section{9 \\ Managing Malaria ${ }^{1}$}

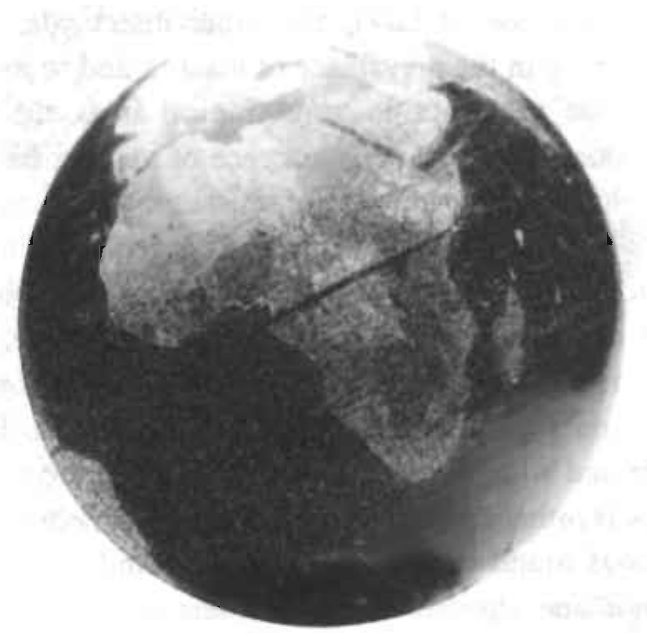

This Chapter is based on Janssen and Martens (1996a+b) and Martens and Janssen (1996). 


\subsection{Introduction}

Malaria is one of the world's most important vector-borne diseases; there are few infectious diseases which have as great an impact on the social and economic development of societies. Out, of a world population of approximately 5,300 million people in 1990, some 2,200 million were regarded as being at risk of contracting malaria, while some 270 million people were actually infected with the malaria parasite. At present, the distribution of malaria is mainly restricted to the tropics and sub-tropics, although before the Second World War, malaria was a relatively common disease in many temperate areas of the world.

Although the effective use of DDT and other insecticides after 1945 led to a significant global decrease in the prevalence of malaria and to its eradication or neareradication in temperate zones and in some tropical areas, the rate of decrease has now slowed down considerably and a resurgence of malaria has occurred in several countries (WHO, 1996). The development of resistance to insecticides is considered to be one of the main obstacles in using insecticides for vector control in any strategy of malaria control/eradication. Resistance to insecticides is most pronounced in regions of Africa, Central America, and West and South-East Asia (Pant, 1988).

A further obstacle is the development of resistance to anti-malarial drugs in $P$ : falciparum, the malaria parasite responsible for most deaths. For many centuries, malaria has been treated with an extract from the bark of the cinchona tree, namely quinine, while a new (synthetic) drug, chloroquine, which became available at the end of World War II, was found capable of preventing and curing malaria, especially since it was less toxic and effective in less frequent doses. By the 1960's, however, plasmodia resistant to chloroquine had emerged, and $P$. falciparum which are resistant to the drug are currently found throughout extensive regions of Africa, South-East Asia, and South America (Figure 9.1). The increased selection and progressive dispersal of parasites resistant to antimalarial drugs is mainly caused by the fact that these preparations are increasingly being used as prophylactics and for self-medication, usually in insufficient doses. The problem of drug resistance has become particularly alarming in Africa, and its continual exacerbation hampers efforts to provide adequate treatment of the disease (Najjera et al., 1992).

It is evident that malaria patterns have, hitherto, depended to a large extent on the effectiveness of control efforts, together with socio-economic development. Although new drugs are being developed and work is progressing on various potential malaria vaccines, given the increasing resistance of the malaria mosquito to insecticides, on one hand, and of its parasite to antimalarial drugs, on the other, the treatment of malaria seems likely to be more problematic in the future. A further factor which may 
influence future malaria trends, and to which attention has only recently been paid, is the projected effect of a human-induced climate change on the transmission dynamics of malaria (Bradley, 1993; Matsuoka \& Kai, 1994; Martin \& Lefebvre, 1995; Martens et al., 1994, $1995 \mathrm{a}, \mathrm{b})$. Anthropogenic climate change may directly affect both the behavior and geographical distribution of the malaria mosquito and the life cycle of the parasite, and, thus, may have implications for the incidence of the disease.

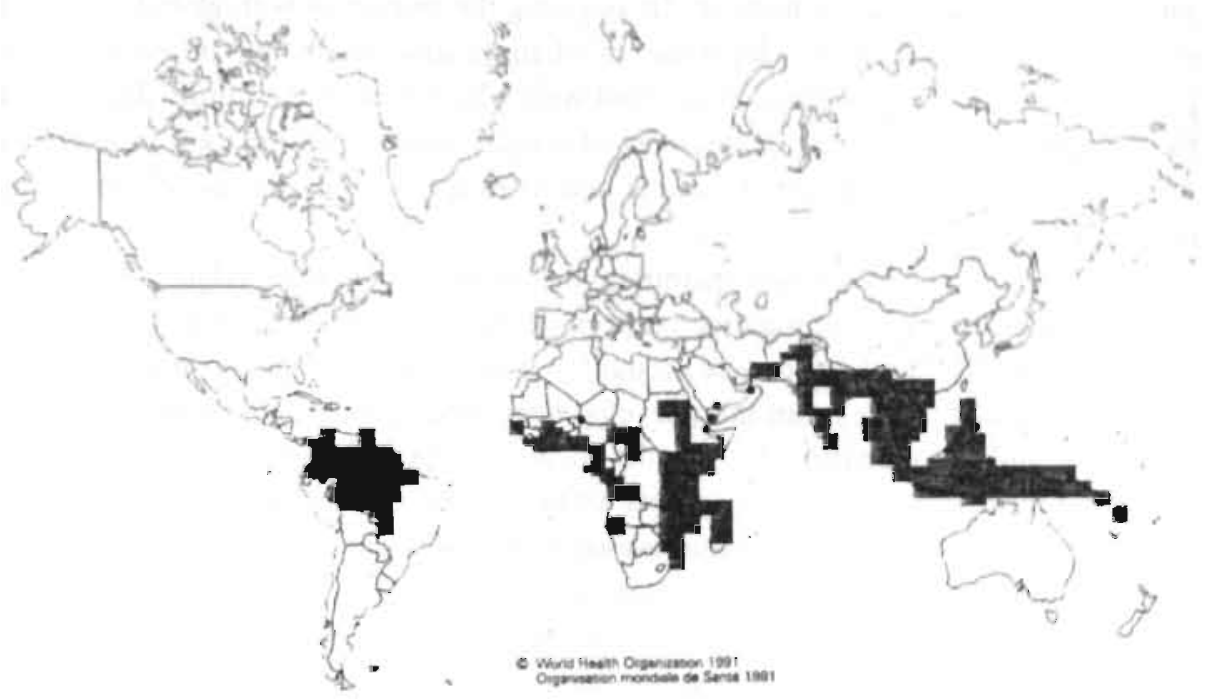

Figure 9.1: Current world-wide distribution where chloroquine-resistant Plasmodium falciparum has been reported (WHO, 1991).

Although models are not intended to replicate all components of a real system, they do prove useful in studying the population dynamics of infectious diseases (Aron and Silverman, 1994). Experimental approaches in the laboratory and in the field are: important, but cannot capture the full range of possible host-parasite interactions. Furthermore, modelling experience leads to the formulation of hypotheses which may inspire experimental research, an example of which would be the model-based hypothesis that inbreeding may accelerate the build-up of drug resistance, which has recently been confirmed empirically in Papua New Guinea (Paul et al., 1995). Over and above enhancing scientific understanding, an important role of malaria models is to support decision-making in the management of malaria control operations. Since the ability of organisms to develop resistance to human interventions has become an important issue in managing malaria, current modelling efforts incorporate the 
adaptation process by adopting either a deterministic or a stochastic approach (e.g. Anderson and May, 1991).

Although the above techniques have been useful, new mathematical tools based on evolutionary processes have appeared during the last decade which are eminently suitable for modelling adaptation. According to Levins (1995), it has become apparent that the classic deterministic approach is incapable of confronting the rapid and unexpected changes on the horizon. In assessing the impact of both global and local changes, the modelling of adaptation to changes and modelling of evolutionary processes themselves provide a crucial tool with which to scan the future. The aim of this Chapter is to discuss the deployment of evolutionary modelling tools in scanning future risks of the occurrence of malaria and assessing possible means of controlling those risks.

A model designed to enhance quantitative projections of climate-related changes in the potential distribution of malaria has been developed by Martens et al. $(1994 ; 1995$ $a, b)$. Although this model does take account of how climate change directly affects the mosquito population, i.e.: mosquito development, feeding-frequency, longevity of the mosquito, and the climatic effect on the incubation period of the malarial parasite inside the mosquito, it does not address artificial interventions by humans and how this may affect the increased malaria risk associated with climate changes. In order to allow for both anti-malarial control measures and the adaptation of mosquitos and parasites to such malaria control policies, the simulation model created by Martens et al. (which describes the transmission dynamics between human and mosquito populations) is combined with genetic algorithms. The latter involves a general and robust evolutionary modelling approach which is based on the mechanics of the survival of the fittest, whereby the inclusion of the notion of variability within the population renders the genetic algorithm a suitable tool for simulating the adaptive behavior of a population within a changing environment. In this Chapter, a simplistic, idealized model of the resistance cycles associated with insecticide and drug use in malaria control programs is presented, together with the impact of climate changes. Although this approach is adopted solely for heuristic purposes, it, nevertheless, does succeed in elucidating the mechanism of resistance development, interactions associated with climate change, and consequences for the implementation of strategies in malaria management. 


\subsection{Malaria}

\subsubsection{Introduction}

Malaria is caused by one or more of four species of parasites of the genus Plasmodium, whereby the vector responsible for malaria transmission is the mosquito of the genus Anopheles. The life cycle of the malaria parasite involves transmission both from mosquito to man and from man to mosquito, effected by the bite of a female mosquito (see Figure 9.2). The parasite multiplies within the mosquito by means of sexual reproduction, and, following an incubation period of several days (depending on the temperature and the species of parasite), malarial parasites can be found in the salivary glands of the insect. When an infected mosquito bites a human host, saliva is also injected and parasites are, thus, transferred to (hitherto uninfected) people, whereupon asexual multiplication takes place in the human host. Following an infective bite, there is an incubation period in the human patient which varies between 10 and 40 days, depending on the species of parasite. The incubation period culminates in a severe attack which is caused by the destruction of infected blood cells and the release of toxins into the bloodstream. Infections involving $P$. falciparum are often associated with fatal complications (e.g. anaemia and cerebral malaria).

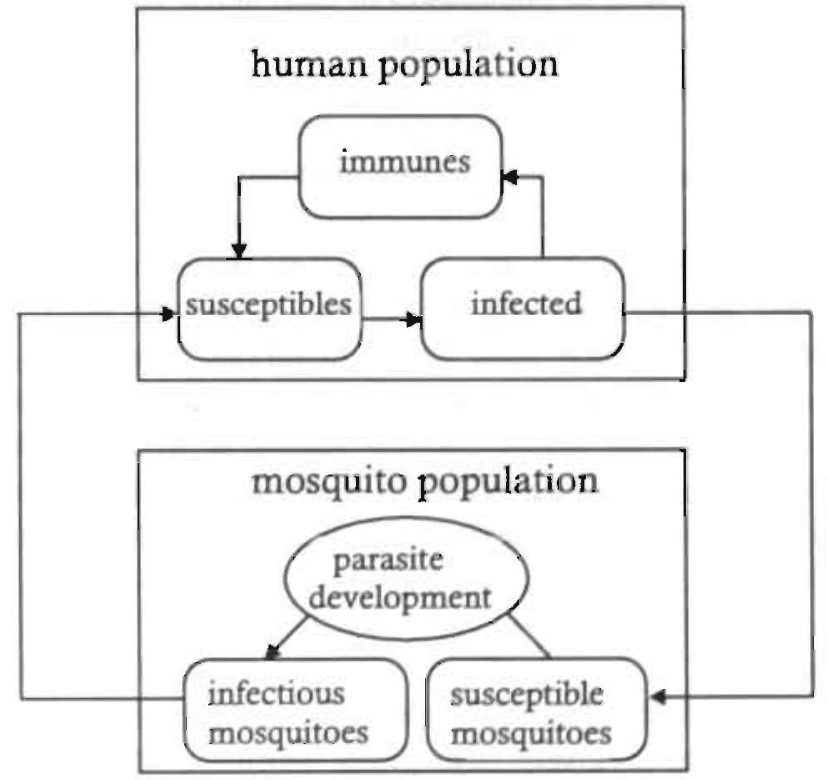

Figure 9.2: Transmission cycle of the malaria parasite. 
Although malaria may manifest itself throughout the world, its incidence shows marked regional variations, these being dependent on four groups of interacting factors, namely: the human host, the malarial parasite, the mosquito as vector, and the environment, whereby the latter term is here understood to subsume physical, biological, and socio-economic elements. These factors will be discussed, in turn, in the sections which follow.

\subsubsection{The Human Host}

Two main factors determine an individual's propensity to succumb to malarial infection: on one hand, genetic factors affect the ability of the parasite to penetrate and maintain itself within the red blood cell; on the other, some degree of immunity may have been acquired by virtue of a previous history of infection. Among the genetic. factors associated with protection against malarial infection are the sickle-cell trait and the Duffy factor (Molineaux, 1988), while those who have survived an attack of malaria acquire some degree of immunity to the disease. The number of parasites in the blood is lower and the infection may present few, if any, clinical symptoms. Consequently, in areas where malaria is rife, deaths from the disease occur mainly in the category of children aged between six months and five years. The high collective levels of acquired immunity of populations in these stable endemic areas reduce the likelihood that malaria epidemics will occur.

\subsubsection{The Parasite}

There are four species of the malaria parasite of the genus Plasmodium, namely: $P$. falciparum, the most common species in tropical areas and the most dangerous clinically; $P$. vivax, which has the broadest geographic range including many temperate zones; and $P$. ovale and $P$. malariae, which are less prevalent (Clyde, 1987). Virulence varies greatly from species to species. The ranking in decreasing order of virulence is as follows: $P$. falciparum, $P$. vivax, $P$. malaria and $P$. ovale. When left untreated, $P$. falciparum does not survive in the human body for longer than two to three years, whereas infection by $P$. vivax may last between two and more than 11 years. The duration of infection by $P$. ovale and by $P$. malariae may last from 1 year up to 53 years.

\subsubsection{The Mosquito}

Malaria is transmitted to humans by female mosquitoes of the genus Anopheles. This mosquito belongs to a very large genus which includes hundreds of species throughout the world, although only 60 of these are actual or potential malarial vectors. There are four distinct stages in the life cycle of the mosquito, namely: the 238 
egg, larval, pupal and adult stages. In order to produce eggs, a female mosquito must take a blood meal, and the eggs are normally laid after the blood meal has been digested. Development and survival during the larval and adult stages of the Anophelines depends on whether temperature, humidity, and rainfall are favorable. Temperature governs the growth rate of a mosquito population by determining the time needed for one generation to develop; the optimum temperature for most malaria vectors is found in the range $20-30^{\circ} \mathrm{C}$. Relative humidities in excess of $60 \%$ are preferred by vectors, and while moderate rainfall may prove beneficial to mosquito breeding, excessive rainfall may flush away the mosquito larvae.

\subsubsection{The Environment}

The physical, biological, and socio-economic aspects of the environment play an essential role in the epidemiology of malaria. Some of the physical factors have already been mentioned above. Among the biological factors which play a role in malaria transmission are the presence of predators and the presence of domestic animals. Cattle may be employed as sources of blood and hence divert mosquitoes from feeding on people. When considering the connections between malaria and socio-economic variables, two apparently contradictory relations can be identified. First, in the long run, social and economic development is associated with a downward trend in malaria, which is partly due to the increased provision of health services, both curative and preventive. Socio-economic development can also suppress malarial prevalence independently of deliberate control measures (e.g. via the enhancement of public hygiene, drainage, and housing) as the cases of Europe and North America would suggest (Bruce-Chwatt, 1980). On the other hand, in the short run, development projects may lead to an increased incidence of malaria, in particular where such projects are divorced from social development (e.g، when they involve deforestation, irrigation, colonization of new territory etc.). It is important to bear in mind that there is a direct feedback from the incidence of malaria to the socioeconomic development of a country. The social and economic damage caused by malaria morbidity and mortality - e.g., declines in the productivity of labor, pressure on health services- may prevent many low-income countries from achieving an efficient level of malaria control and/or an eradication program.

\subsubsection{Climate Change Impacts}

Direct effects of the anticipated changes in global and regional temperature, precipitation, humidity, and wind patterns resulting from anthropogenic climate change are factors which have an impact on the mosquito reproduction habits and on their longevity, and are, thus, associated with changes in annual vector density. In 
general, the rate of development of a parasite accelerates as the temperature rises. An increase in temperature may, therefore, result in the completion of the life cycle of a parasite in areas in which previous temperatures were too low for the parasite to reach maturity. Indirect effects of climate change include changes in vegetation and agricultural practices which are mainly caused by temperature changes and trends in rainfall patterns. Another indirect effect of climate change is associated with the rise in sea level and the resulting coastal flooding. The proliferation of brackish water lagoons influences the availability of habitat and either encourages or discourages vector species, depending on whether or not they prefer brackish water. Generally speaking, drought and desertification, including a migration or extension of global desert belts, could be expected to decrease malaria transmission. It is, thus, evident that major changes in the incidence of this vector-borne disease might be expected to be associated with a climate change.

\subsubsection{The History of Modelling Malaria}

The history of a mathematical approach to malaria is nearly as old as the discovery of its transmission dynamics. The earliest attempt to arrive at quantitative understanding of the dynamics of malaria transmission was made by Ross (1911). His models consist of a handful differential equations which. describe changes in the densities of susceptible and infected people and mosquitoes. In the 1950's, MacDonald (1957) added a dimension of biological realism to these early models by his careful attention to the interpretation and estimation of parameters. Although these basic models provide a useful overview of the dynamics of malarial infection, many of their predictions deviate strikingly from reality.

An obvious modification to the basic model would be the incorporation of latent periods, during which hosts are infected, but are not yet capable of transmitting the disease. Furthermore, models addressing the transmission dynamics of malaria have begun to take account of the phenomenon of acquired immunity. The reason for this belated attention to immunity development is in part a consequence of the early focus in malaria models on the vector component in transmission, which is, in turn, explained by the initial aim of global eradication of malaria by means of the application of DDT. Aron and May (1982) have since then described a simple manner in which to incorporate the observed mechanism of the maintenance of immunity with continuous exposure. Although their model represents an advance over the earlier simple models, it, nevertheless, remains a very crude approximation of the true complexities of immunity to malarial infection.

More elaborate assumptions for the malaria parasite in the vector and in the human host are made by the Garki model (Dietz et al., 1974; Molineaux et al., 1978). A 240 
number of models have been developed to estimate age-and time dependent infection and recovery rates (see e.g. Nedelman, 1985).

\subsubsection{Modelling Resistant Development}

Most of the mathematical models which estimate the effect of eradication programs on malaria transmission have not yet included the ability of organisms to develop resistance to drugs or insecticides (e.g. Nájera, 1974; Tabashnik, 1986; Collet \& Lye, 1987), although the evidence gathered in the field proves that this is a serious omission. With respect to resistance dynamics, a number of simulation models have contributed to our general understanding of this phenomenon and the development of strategies to reduce the development of resistance (a review of these can be found in Glass et al., 1984). Most of modellers have postulated that resistance is determined by a single gene, so that these models are generally not applicable when resistance is a quantitative trait (polygenic), in which the underlying genes are not identified individually. Curtis and Otoo (1986) emphasized the role of linkage between two resistance genes, resulting in a selective build-up of double resistance among insects in response to simultaneous exposure to two different insecticides, and they employed a similar approach to model the development of resistance to drugs among Plasmodium parasites.

Because insecticide and anti-malarial drugs are agents of selection, resistance to them can be studied using the same theoretical frameworks as those which have been applied to other types of evolutionary change. In the sections which follow, the development of resistance is charted by means of genetic algorithms, a tool to simulate complex adaptive systems. 


\subsection{Modelling Malaria: a Complex Adaptive Systems Approach}

\subsubsection{Introduction}

The model described in this Chapter is an extension of the systerns approach previously adopted by Martens et al. (1994; 1995a,b), and addresses two general malaria control options, namely: the use of insecticides to decrease mosquito densities, and the use of drugs to suppress the viability of parasites. While Martens et al. intended to create a global model of the effects of an anthropogenically-induced climate change on malaria risk, the model presented here aims at incorporating local dynamics, in order to derive a generic local model which takes account of human intervention in terms of insecticide and drugs use and the development of resistance to these control measures.

\subsubsection{The Malarial System}

A simple representation of the malarial system is the following. The mosquito system is represented by $X$, the vectorial capacity without taking account of the effect of temperature change. This vectorial capacity is defined as the number of potentially infective contacts inflicted by the mosquito population per infectious person per day. The human population can then easily be described by the categories susceptible $\left(\mathrm{y}_{1}\right)$, infected $\left(\mathrm{y}_{2}\right)$ and immune $\left(\mathrm{y}_{3}\right)$. If different age classes, births, and natural deaths are ignored, then the human population can be represented by the following three equations:

- The number of susceptible changes when immune people lose their immunity at rate $\left(r_{3}\right)$ and if susceptible people become infected (at rate $r_{1}$ ):

$$
d y_{1} / d t=r_{3} \cdot y_{3}-r \cdot y_{1}
$$

- The number of infected people changes when susceptible persons become infected and if infected people lose their infection at rate $r_{2}$ and become immune or die from infection (at rate $\mu_{1}$ ):

$$
d y_{2} / d t=r_{1} \cdot y_{1}-\left(\mu_{1}+r_{2}\right) \cdot y_{2}
$$

- The number of immune people changes due to infected people who become immune and immune people who lose their immunity: 


$$
d y_{1} / d t=r_{2} \cdot y_{2}-r_{3} \cdot y_{3}
$$

The yearly rate of infection depends on the vectorial capacity, $x$, times number of days in a year, 365 , and the portion of infected people:

$$
r_{1}=365 \cdot x \cdot y_{2} /\left(y_{1}+y_{2}+y_{3}\right)
$$

Assume that infection lasts for some fixed period of time $1 / b_{1}$, in the absence of reexposure, but that if a person is further exposed before $1 / b_{1}$ units have elapsed, infection is sustained and another interval of duration $1 / b_{1}$ without reinfection is required before infection is lost (Anderson and May, 1991). If infection occurs at per capita rate $r_{1}$, the average time spent in the immune state can be calculated as a function of $\mathrm{r}_{1 .}$. The resulting average per capita loss of infection, is

$$
\text { if } r_{1}=0 \text { then } r_{2}=b_{1} \text { else } r_{2}=r_{1} /\left(e^{r_{1}}-1\right)
$$

The loss of immunity is simulated in the same way as the loss of infection with the use of a fixed time period $b_{2}$ :

$$
\text { if } r_{1}=0 \text { then } r_{3}=1 / b_{2} \text { else } r_{3}=r_{1} /\left(e^{r_{3} b_{4}}-1\right)
$$

The interaction between the human population and the mosquito population determines the transition rates among susceptible, infected, and immune populations, respectively. To this end, the mosquito system is denoted by state variable $\mathrm{x}$ and the human system by state variable $y(t) \in \mathrm{R}^{7}$. The potential of the mosquito population to transmit $P$. falciparum? is in the model assumed to be influenced by temperature, $\mathrm{T}(\mathrm{t})$, and by the use of insecticides, $\mathrm{u}_{1}(\mathrm{t})$. The dynamics within the human population are affected by the transmission potential of the mosquitoes and by the use of antimalarial drugs, $\mathrm{u}_{2}(\mathrm{t})$.

$$
\begin{aligned}
x(t) & =f\left(T, u_{i}\right) \\
\frac{d \mathrm{y}(t)}{d t} & =g\left(\mathrm{y}, x, u_{2}\right)
\end{aligned}
$$

I have focused on the transmission dynamics of $P$. falciparum since it is the most lethal malaria parasite and is exhibiting world-wide development of resistance to anti-malarial drugs. 
In order to incorporate adaptation to antimalarial drugs and insecticides, this dynamic system is coupled to genetic algorithms which enable the genetic variety within the mosquito population and the parasite population (Figure 9.3). The genetic algorithms determine parameters that, in turn, determine the resistance of the mosquitoes and parasites and the optimum temperature for mosquito survival. The system can, therefore, be reformulated as

$$
\begin{aligned}
& x(t)=f\left(T^{a}, u_{1}^{a}, T, u_{i}\right) \\
& \frac{d \mathrm{y}(t)}{d t}=g\left(\mathrm{y}, x, u_{2}^{a}, u_{2}\right)
\end{aligned}
$$

where $T ", u_{1}{ }^{2}$ and $u_{2}{ }^{2}$ represent fixed parameters in system (9.7). $T^{*}$ represent the change of the optimal temperature with respect to the reference value, $u_{1}{ }^{2}$ and $u_{2}{ }^{a}$ represent the degree of resistance (see Section 9.3.3). Now that they are simulated by genetic algorithms, they are subject to adaptations, if temperature changes, or if insecticides and antimalarial drugs are used. In Sections 9.3.2.1 and 9.3.2.2, this original model representation (9.8) will be described, and in Section 9.3.3 the implications of the incorporation of the genetic algorithms are discussed.

Dynamic System

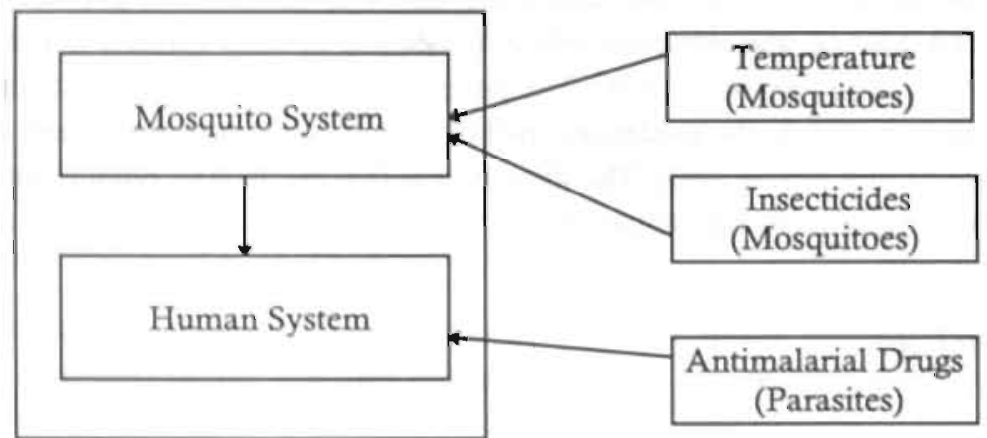

Figure 9.3: Simplified scheme of the integration of a system dynamic model with genetic algorithms. 


\subsubsection{The Mosquito Population}

The (infection-related) dynamics of the mosquito population proceed much more rapidly than do human population dynamics, so that the mosquito system can be considered as being in equilibrium with respect to changes in the human population. Therefore, the description of the mosquitoes is given in terms of an equilibrium instead of in a set of differential equations. Following Garrett-Jones (1964), the entire mosquito population is incorporated in a single state variable, namely: vectorial capacity. The formulation of vectorial capacity used by Martens et al. is multiplied by the relative fitness of mosquitoes to insecticides, $\mathrm{F}^{\mathrm{m}}$ (see Section 9.3.3.1) resulting in a formulation of vectorial capacity which includes the impact of the use of insecticides. Furthermore, an adaptive representation of survival probability can be used to describe the adaptation of a mosquito population to a change in temperature (see section 9.3.3.1):

$$
x=\frac{\beta_{i} \cdot z_{i}^{2} \cdot z_{2}^{5}}{-\ln \left(z_{2}\right)} \cdot F^{m}\left(u_{1}\right)
$$

where $\mathrm{x}$ is the vectorial capacity; $B_{1}$ incorporates variables assumed to be temperature independent (including the efficiency with which a mosquito infects a susceptible human; the propensity of the mosquito population to feed on humans; and the density of the mosquito population in relation to man). The term $z_{1}$ represents the man-biting habit (number of blood meals taken from humans per mosquito per day); $z_{2}$ is the daily survival probability of the mosquito; and $z_{3}$ is the incubation period of the parasite in the vector (in days).

The man-biting habit depends on the frequency with which one vector takes a blood-meal and the total number of these blood meals being taken from man. The frequency of feeding depends mainly on the rapidity of digestion of a blood-meal, a rate which increases as temperature rises so that at the optimum temperature, one meal is taken every 48 hours (Muirhead-Thompson, 1954). The relation between temperature and the rapidity of blood digestion is given in Detinova (1963). The resulting equation for the man-biting habit (per day) is:

$$
z_{1}=\frac{T-\beta_{3}}{\beta_{2}}
$$


where $B_{2}$ is the number of "degree-days" required for the digestion of a portion of ingested blood, (36.5 degree-days at relative humidity $70-90 \%$ ), $B_{3}$ is the minimum temperature required for the digestion of the blood meal $\left(9.9^{\circ} \mathrm{C}\right)$ and $\mathrm{T}$ is the actual average temperature (in ${ }^{\circ} \mathrm{C}$ ).

The vector's longevity determines its ability to transmit a parasite, since the female mosquito has to live long enough for the parasite to complete its development. There is presumably an optimum temperature and an optimum humidity for each species of mosquito, and it is apparent that, between certain limits, longevity decreases as temperature rises, and increases as relative humidity rises (Boyd, 1949; Molineaux, 1988). Data reported by Boyd (1949) and Horsfall (1955) on mosquito longevity indicate an optimum temperature of about $20-25^{\circ} \mathrm{C}$ and an optimum relative humidity of $60-90 \%$, and the assumption about the relation between the longevity of the Anopheles mosquito and temperature is based on these data. The maximum mean longevity is assumed to be 10 days $\left(z_{2}=0.9\right)$ at temperatures of about $20^{\circ} \mathrm{C}$. The assumed (non-adaptive) relationship between temperature and daily survival probability of the adult mosquito is written as follows (Martens et al., 1994) ((see section 9.3.3.1) for an adaptive representation)):

$$
z_{2}=\exp \left(\frac{-1}{-4.4+1.31 \cdot T-0.03 \cdot T^{2}}\right)
$$

The incubation period (duration of sporogony) in the vector must have elapsed before the infected vector can transmit the parasite. The duration of this latent period depends on two critical factors: species of parasite and ambient temperature. The parasites develop in the vector only within a certain temperature range, and whereas the minimum temperature for parasite development lies between 16 and $19^{\circ} \mathrm{C}$ in the case of $P$. falciparum, the proportion of parasites surviving decreases rapidly at temperatures over $32-34^{\circ} \mathrm{C}$ (Horsfall, 1955; MacDonald, 1957; Detinova, 1963). The relation between the incubation period and temperature (if higher than $16^{\circ} \mathrm{C}$ ) can be expressed in the following equation (MacDonald, 1957):

$z_{3}=\frac{\beta_{4}}{T-\beta_{3}}$

where $z_{3}$ is the incubation period of the parasite inside the vector (in days), $B_{4}$ the number of "degree-days" required for the development of the parasite (=111 degreedays for $P$. falciparum (Detinova, 1963)), T the actual average temperature (between $B_{5}$ and a maximum temperature of about $40^{\circ} \mathrm{C}$; in ${ }^{\circ} \mathrm{C}$ ), and $B_{5}$ the minimum temperature required for parasite development $\left(16^{\circ} \mathrm{C}\right.$ for $P$. falciparum). 


\subsubsection{The Human Population}

The model used to describe the transition between the reservoirs of the human population at risk is based on a microparasite-epidemiological model as described in Aron and May (1982), Bailey (1982), Levin et al. (1989), and Anderson and May (1991). The human population subject to a risk of malaria is divided into three categories for each of two different age classes $(i=1$; children younger than 5 , and $i=2$; people of 5 years and older). The three categories are susceptible persons $\left(y_{1}{ }^{\prime}\right)$, infected persons $\left(y_{2}\right)$, and immune persons $\left(y_{3}{ }^{\prime}\right)$. The latent reservoir is omitted, because the duration of a stay in this reservoir is usually very short in comparison to the residence time in the other reservoirs. The total population is represented by $y_{4}$, which gets children with the birth rate $\lambda$.

The number of susceptible persons may change over time, as they become members of the infected class at a rate $\mathrm{r}_{1}$. Infected individuals either die from infection at a rate $\mu_{1}^{(1)}$ or recover to join the immune category (at a rate $r_{2}$ ). Immune persons lose their immunity at a rate $\mathrm{r}_{3}$, and those who have lost their immunity return to the reservoir of susceptible persons. All newborn babies are assumed to be members of the category of susceptibles; as they grow older, they graduate from the younger age class to the older (at a rate $\lambda_{a}$ ). People die from other causes at a rate $\mu$.

The dynamic behavior of the human system can be described thus (see also Figure 9.4):

$d y / d t=M \cdot y$

with $\mathrm{y}=\left[y_{1}^{(1)}, y_{1}^{(2)}, y_{2}^{(1)}, y_{2}^{(2)}, y_{3}^{(1)}, y_{3}^{(2)}, y_{4}\right]$

and

$M=\left[\begin{array}{ccccccr}-r_{1}-\mu-\lambda_{3} & 0 & 0 & 0 & r_{2} & 0 & \lambda \\ \lambda_{3} & -r_{1}-\mu & 0 & 0 & 0 & r_{2} & 0 \\ r_{1} & 0 & -\mu-\mu_{1}^{(1)}-\lambda_{2}-r_{2} & 0 & 0 & 0 & 0 \\ 0 & r_{1} & \lambda_{2} & -\mu-\mu_{1}^{(2)}-r_{2} & 0 & 0 & 0 \\ 0 & 0 & r_{2} & 0 & -r_{3}-\mu-\lambda, & 0 & 0 \\ 0 & 0 & 0 & r_{2} & \lambda_{2} & -r_{3}-\mu & 0 \\ 1 & 1 & 1 & 1 & 1 & 1 & 0\end{array}\right]$

where $r_{1}$ is the rate of infection, $r_{2}$ the rate of loss of infection and $r_{3}$ the rate of loss of immunity. 
the modelling approach to the issue of resistance development. The model should, therefore, be seen as a heuristic device in which expert knowledge is integrated in such a manner that interactive and transparent simulations and experiments can be performed.

\subsubsection{The Mosquito}

With the help of the genetic algorithm, sexual reproduction is implemented using the two genetic parameters: namely the crossover probability $\left(p_{c}\right)$ and the mutation probability $\left(\mathrm{p}_{\mathrm{m}}\right)$. To simulate the adaptation of mosquitoes, a crossover probability of 0.4 and a mutation rate of 0.001 were assumed. These values are consistent with those generally used in genetic algorithm applications, and the results are not sensitive to this assumption (Section 9.4.7).

The fitness of a (biological) population is related to the chance of its members begetting descendants (Hofbauer and Sigmund, 1988). The expected lifetime of a mosquito is assumed to be a measure of individual fitness, since life expectancy is directly related to the production of offspring. Having adopted this approach, I distinguish two pressures on the mosquito population, namely temperature change and insecticide use. I assume that adaptation to temperature change and to insecticides are independent of each other.

\section{Adaptation to Temperature Change}

For every mosquito, a temperature level is assumed at which its expected lifetime would be maximized (Figure 9.5), but within the mosquito population there is variation of these optima among individuals. If the temperature increases over a longer period (say various years), mosquitoes for which the optimum is higher than average exhibit greater fitness. Due to the mechanisms associated with the "survival of the fittest", the average optimum temperature for longevity will, therefore, rise ${ }^{3}$. The implementation of this process by means of a genetic algorithm proceeds as follows.

Within the mosquito system, the daily probability of survival is a function of temperature (see eq.9.11). Within the population, individual temperature optima are scattered around the mean temperature. For simplicity, no distinction is made in seasonal temperature changes. The daily survival probability can, therefore, be treated

\footnotetext{
${ }^{3}$ I recognize that this account of adaptation to unfavorable temperatures is just one of the possibilities. Another would be the migration of mosquitoes to micro-habitats where temperatures are more suitable.
} 
as a function of the local mean temperature, whereupon the variable $\mathrm{T}_{\mathrm{i}}^{\mathrm{a}}$ is introduced which represents the individual adaptation to temperature. This results in a daily survival probability so that the fitness function of mosquito $i$ becomes,

$F_{T, i}=-4.4+1.31 \cdot\left(T-T_{i}^{a}\right)-0.03 \cdot\left(T-T_{i}^{a}\right)^{a}$

If temperature $\mathrm{T}$ changes, the value of $\mathrm{T}_{i}^{*}$ will also change since the "survival of the fittest" keeps the mosquitoes in the optimum temperature zone.

Furthermore, the daily survival probability of the adult mosquito becomes:

$z_{2}=\exp \left(\frac{-1}{-4.4+1.31 \cdot\left(T-T^{a}\right)-0.03 \cdot\left(T-T^{a}\right)^{2}}\right)$

where $T^{*}$ is the mean of $T_{i}^{*}$.

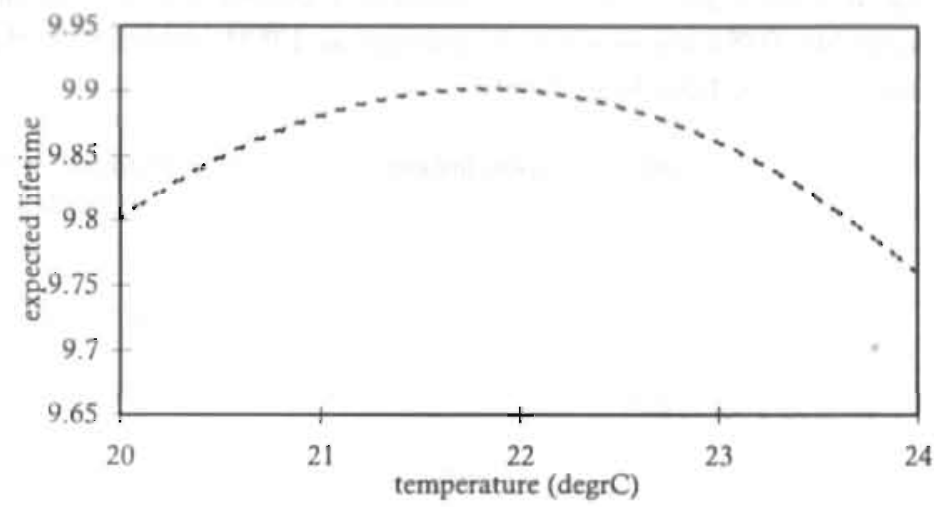

Figure 9.5: Life expectancy (in days) as a function of temperature (equation 9.11).

\section{Adaptation to Insecticides}

An important human-induced pressure on the mosquito population is the use of insecticides. Several models have been developed to enable us to understand and manage the evolution of insecticide resistance, and nearly all of them assume that resistance is controlled by two alleles at one locus (Taylor, 1983; Anderson and May, 1991). However, the fitness function is based on the study published by Tabashnik (1990), who has investigated three- and four-allele models.

A three-allele model is simulated by distinguishing three kinds of mosquitoes, namely: susceptibles, moderately-resistant, and resistant individuals, taking them as. three classes of individual sensitivity to insecticides. The assumption is that a certain dose of insecticide reduces fitness in the manner depicted in Figure 9.6, whereby it is. 
assumed that the same dose would have a more pronounced impact on susceptible mosquitoes than on (moderately) resistant ones. The fitness function expresses the notion that the fitness of the three classes drops in a decreasing rate for a higher dose of insecticides (Schapira, 1990). Obviously, if alternative insecticides are applied which affect the three categories differently for some reason, for example being more effective, the results and conclusions may differ.

In addition, the simulation incorporates a "biotic fitness" component which represent the relative fitness of the mosquito, in the event of no insecticides being used at all. A lower value for the biotic fitness of the more resistant genes explains the lower density of these genes in an insecticide-free environment. Given an initial random distribution, the following table is derived for the fitness of mosquitoes, $\mathrm{F}^{\mathrm{m}}$, to which a certain dose of insecticides $u_{1}$ is applied, whereby I assume that $99 \%$ of the mosquitoes are susceptible, $0.9 \%$ are moderately-resistant and $0.1 \%$ resistant in the initial situation (values based on Tabashnik, 1990).

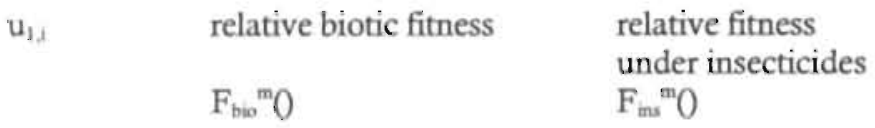

$\begin{array}{llll}\text { susceptible } & {[0.0,0.99)} & 1.0 & 1-\mathrm{u}_{1} /\left(0.002+\mathrm{u}_{1}\right) \\ \text { moderately-resistant } & {[0.99,0.999)} & 0.95 & 1-\mathrm{u}_{1} /\left(0.05+\mathrm{u}_{1}\right) \\ \text { resistant } & {[0.999,1.0]} & 0.9 & 1-\mathrm{u}_{1} /\left(0.15+\mathrm{u}_{1}\right)\end{array}$

The average fitness of the individuall mosquitoes $\mathrm{F}^{\mathrm{m}}\left(\mathrm{u}_{1,0}\right)=\mathrm{F}_{\text {bio }}{ }^{\mathrm{m}}\left(\mathrm{u}_{1, \lambda}\right)^{\star} \mathrm{F}_{\mathrm{ms}}{ }^{\mathrm{m}}\left(\mathrm{u}_{1, \lambda}\right), \mathrm{F}_{\mathrm{ins}}{ }^{\mathrm{m}}\left(\mathrm{u}_{1}\right)$, is used in the equation for vectorial capacity $\mathrm{x}$.

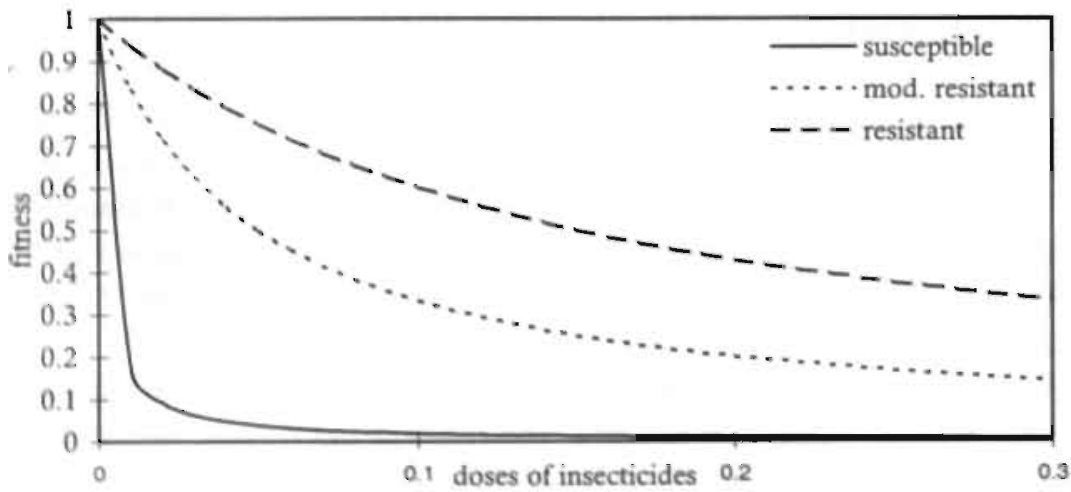

Figure 9.6: Relative fitness of mosquitoes related to the use of insecticides. A certain dose of insecticides leads to a reduction in fitness which is more severe in the case of susceptible than resistant individuals. 


\subsubsection{The Parasite}

The dynamics of the gene pool in parasites differ from those in the mosquitoes. Since, the population of parasites is spread among the human population and the mosquito population, the transmission of resistant parasites through a vector population to other human hosts limits the efficacy of adaptation in the parasite population at large. Note that a single gene pool for parasites is assumed, although several local clusters do exist (in the hosts). In view of the lack of data, I have been obliged to use the same crossover and mutation probabilities as for the mosquitoes in the reference runs.

\section{Adaptation to Drugs}

Having established the modelling approach to the resistance among mosquitoes to the use of insecticides, the adaptation of parasites to the use of antimalarial drugs is modelled in a similar manner. Thus " a three-allele model is simulated by distinguishing three kinds of parasites, namely: susceptibles, moderately-resistant, and resistant individuals, and taken these as three classes of individual sensitivity to the drugs involved. Given an initial random distribution, the following table is derived for the fitness of parasites to which a certain dose of drugs $\mathrm{u}_{2}$ is applied, whereby it is assumed that $99 \%$ of the population is susceptible, is $0.9 \%$ moderately-resistant, and $0.1 \%$ resistant in the initial situation:

\begin{tabular}{|c|c|c|c|}
\hline & $\mathrm{u}_{2, i}$ & relative biotic fitness & $\begin{array}{l}\text { relative fitness } \\
\text { under drugs }\end{array}$ \\
\hline & & $\mathrm{F}_{\mathrm{bio}}{ }^{p}$ & $F_{d t}{ }^{p}$ \\
\hline usceptible & {$[0.0,0.99)$} & 1.0 & $1-\mathrm{u}_{2} /\left(0.002+\mathrm{u}_{2}\right)$ \\
\hline moderately-resistant & {$[0.99,0.999)$} & 0.95 & $1-\mathrm{u}_{2} /\left(0.05+\mathrm{u}_{2}\right)$ \\
\hline resistant & {$[0.999,1.0]$} & 0.9 & $1-\mathrm{u}_{2} /\left(0.15+\mathrm{u}_{2}\right)$ \\
\hline
\end{tabular}

The fitness function for a parasite, therefore, becomes

$F^{p}\left(u_{2}\right)_{i}=F_{b i o, i}^{p} \cdot F_{d r, i}^{p}$

In equation (9.8) the mean value of $\mathrm{F}^{p}\left(\mathrm{u}_{2, j}\right), \mathrm{F}^{\mathrm{P}}\left(\mathrm{u}_{2}\right)$, is used to determine the impact of resistance on the transmission dynamics within the human population. It should be noted, however, that in some places biological advantage of chloroquine-resistant $P$. falciparum has been observed (discussed by Wernsdorfer, 1994). This would imply 
that resistance development would proceed more rapidly then under the assumption discussed above.

\subsubsection{Migration and Refugees Among Mosquitoes and Parasites}

Georghiou and Taylor (1977) argued that the migration of insects tends to delay the rate of evolution of resistance. In addition, the percentage of mosquitoes or parasites not reached by the anti-malarial treatment (the so-called refugees) will inevitably influence resistance development. The complex adaptive systems approach takes account of both of these processes in the development of resistance, among mosquitoes as well as among parasites.

It would seem self-evident that, depending on landscape and infrastructure, mosquitoes are more or less able migrate from place to place, and that mosquitoes susceptible to insecticides may, thus, enter a treated area. Moreover, parasites susceptible to anti-malarial drugs can also migrate, whether they are carried by mosquitoes or humans. Migration is modelled by assuming that during each timestep a fraction of the new population is bred under the initial conditions, i.e. not yet adapted to the changed conditions.

Insecticides are sprayed on specific areas so that $100 \%$ coverage is seldom achieved. Drugs are not taken (sufficiently) by all humans, so that a fraction of the parasites escapes from it. This phenomenon of refugees is modelled by assuming that during each timestep, a part of the population, the size of which is randomly selected, has not been treated despite the control programs which have been implemented.

\subsection{The Experiments}

\subsubsection{Introduction}

The experiments deal with the consequences of the use of insecticides and antimalarial drugs, together with a temperature change on the occurrence of malaria for a time horizon of one decade, using time steps of 0.1 year, in two types of regions: a region of low endemicity, and a region of high endemicity. Although not the real generational longevity among the parasites and mosquitoes is specified, the time horizon is based on observed time elapsed in acquiring resistance (see Table 9.1). Furthermore, it is assumed that the initial force of infection $\left(r_{1}\right)$ is 2.0 per annum in highly endemic regions and 0.1 in areas of lower endemicity (Martens et al., 1995a). These values were chosen since they lie within the range of the values reported in 
several studies on the pristine force of infection among young children. The initial settings for these systems are given in Table 9.2. Areas of lower endemicity can be characterized as exhibiting low vectorial capacity resulting in a high percentage of susceptible persons $(=80 \%)$, and low percentages of infected $(=8 \%)$ and immune persons $(=12 \%)$. Areas of low endemicity vis-à-vis $P$. falciparum can be found in South-East Asia and South America. Regions of high endemicity are characterized by a relatively high vectorial capacity. In the initial situation, there is a high percentage of immune $(-68 \%)$ and infected persons $(-27 \%)$. The younger age class especially suffers from a high percentage of infected persons(- $45 \%)$. Highly endemic regions are mainly found in tropical Africa.

I now propose to report a set of results which I have derived using the complex adaptive systems approach. In the starting year, the situation is assumed to be near equilibrium. This assumption about an equilibrium state is made for analytical purposes, namely, to render the impact of control policies and temperature change on the occurrence of malaria, transparent, thereby including the adaptation of mosquitoes and parasites. Therefore, I have assumed a steady state situation in demographic, social, and economic development, although I recognize that these factors may influence future developments of malaria.

The results are presented as time series covering a period of 10 years. In view of the stochastic elements of the model, I elected to use a large number of runs (100)", and determine the mean and the extremes of important indicators. This procedure yields ranges of uncertainty, whereby the uncertainty does not lie in the different parameter values of the model, but rather in the stochastic characteristics and the complexity of the system.

In the interest of analytical lucidity, two broad control levels for both insecticides and antimalarial drugs are distinguished, namely: the low and the high dose. In case of a low dose, a value of $u_{i}$ equal to 0.002 is adopted, which represents a $50 \%$ deterioration in the fitness of susceptible mosquitoes or parasites. The high dose $u_{i}$ is assumed to be equal to 0.05 such that the fitness of the moderately-resistant mosquito or parasite decreases by $50 \%$.

A typical outcome is shown in Figure 9.7, which shows the impact of using a low dose of insecticides. Although the input variables are the same for the 100 runs, there is a large spread in the optimal temperature for the mosquitoes, the vectorial capacity, and the incidence of malaria. Although on average the use of a low dose of

\footnotetext{
"Experiments showed that a higher number of runs would not affect the mean values significantly.
} 
insecticides leads to an increase in the incidence of malaria in the long-run, it might also lead to a slow decrease of the incidence, if evolutionary adaptation among mosquitoes proceeds very slowly. In order to envisage the trends for the various sensitivity tests, I will confine myself to depicting the average scores in the following subsections.

Table 9.1:The time which elapses before a majority (i.e.> 50 per cent) of the in dividuals in the mosquito population become resistant to the control agent (source: Anderson and May, 1991).

\begin{tabular}{|l|l|l|}
\hline $\begin{array}{l}\text { Anopheline mosquitoes } \\
\text { (different localities) }\end{array}$ & Control agent & $\begin{array}{l}\text { Time to resistance } \\
\text { (in years) }\end{array}$ \\
\hline An. sacharovi & DDT & $4-6$ \\
& dieldrin & 8 \\
An. maculipennis & DDT & 5 \\
An. stephansi & DDT & 7 \\
& dieldrin. & 5 \\
An. culicifacies & DDT & $8-12$ \\
An. annuaris & DDT & $3-4$ \\
An. sundaicus & DDT & 3 \\
& dieldrin. & $1-3$ \\
An. quadrimaculatus & DDT & $2-7$ \\
& dieldrin & $2-7$ \\
An. pseudopunctipennis & DDT & $>20$ \\
& dieldrin & 18 weeks \\
\hline
\end{tabular}



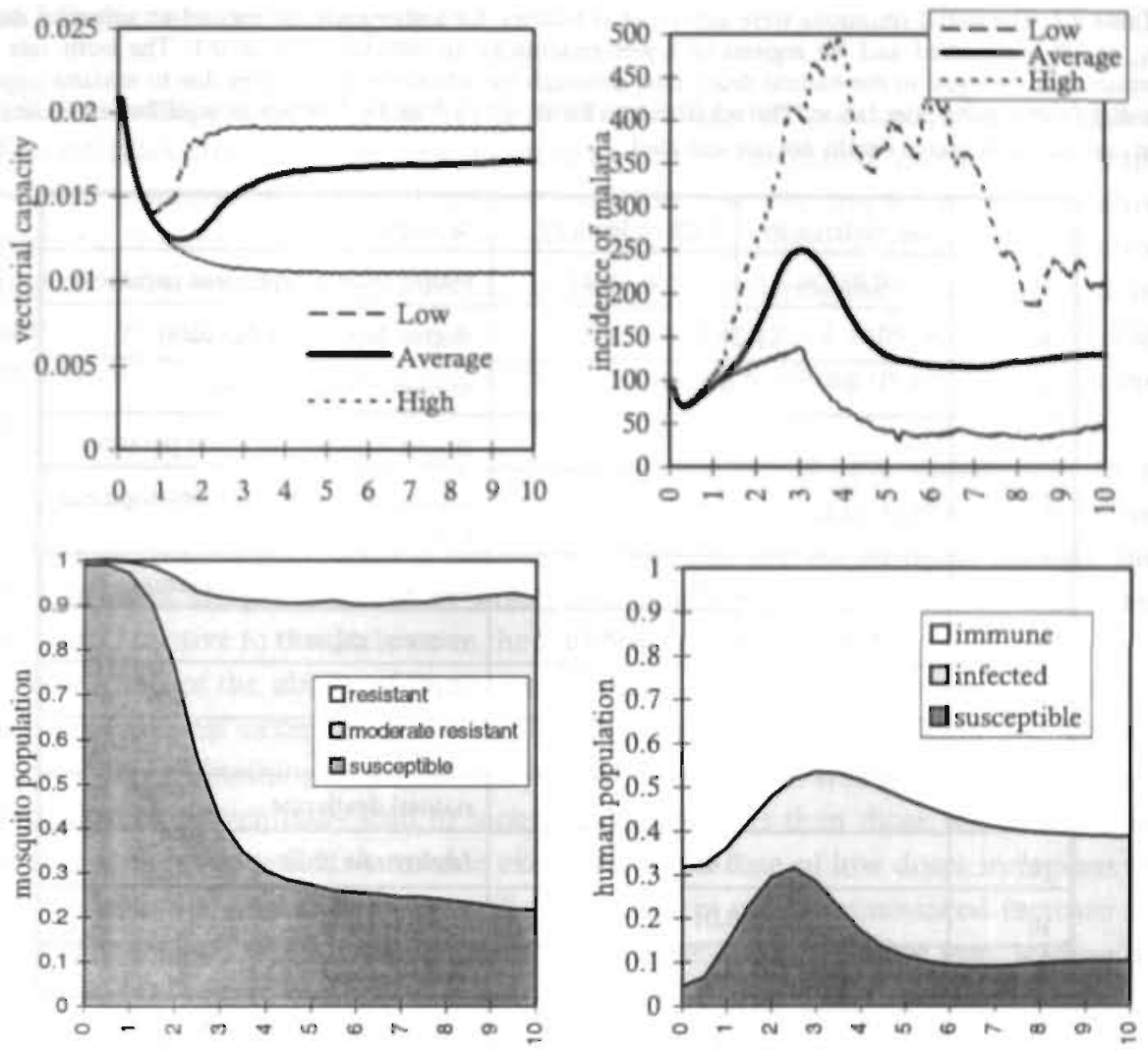

Figure 9.7: An example of an experiment in a region of high endimicity. Depicted are the average and extremes, in the first two pictures, and fraction of resistant, moderate resistant and susceptible mosquitoes, and average fraction of immune and susceptible people is the last two pictures, for a sample of 100 runs. 
Table 9.2: The initial situations were arrived at as follows: for highly endernic regions an infection rate, $r_{1}$, of 2.0 is assumed and for regions of lower endemicity an infection rate of 0 .I. The birth rate is assumed to be equal to the natural death rate, although the additional death rates due to malaria imply a slightly declining population. The initial values for $B_{1}, y_{1}{ }^{\infty}, y_{2}{ }^{6}$ and $y_{3}{ }^{6}$ reflect an equilibrium situation in case malaria-related deaths are not included.

\begin{tabular}{|c|c|c|c|}
\hline & low endemicity & high endemicity & description \\
\hline$B_{1}$ & 0.02226 & 0.13445 & temperature independent parameter \\
\hline $\mathrm{B}_{2}$ & \multicolumn{2}{|r|}{36.5} & degree days blood digestion \\
\hline B) & \multicolumn{2}{|r|}{9.9} & minimum temperature \\
\hline$B_{4}$ & \multicolumn{2}{|r|}{111} & degree days development parasites \\
\hline Bs & \multicolumn{2}{|r|}{16.0} & $\begin{array}{l}\text { minimum temperature development } \\
\text { parasites }\end{array}$ \\
\hline $\mathrm{T}$ & \multicolumn{2}{|c|}{21.88} & initial local mean temperature \\
\hline $\mathrm{x}$ & 0.00335 & 0.02018 & vectorial capacity \\
\hline$\lambda$ & \multicolumn{2}{|r|}{0.02} & birth rate \\
\hline$\lambda_{2}$ & \multicolumn{2}{|r|}{0.2} & aging children \\
\hline$\mu$ & \multicolumn{2}{|r|}{0.02} & natural death rate \\
\hline$\mu_{i}^{\prime}$ & \multicolumn{2}{|r|}{0.04} & fatality rate $(0-5)$ \\
\hline$\mu_{1}^{2}$ & \multicolumn{2}{|r|}{0.01} & fatality rate $(>5)$ \\
\hline$b_{1}$ & \multicolumn{2}{|r|}{1} & basic loss rate infection \\
\hline$b_{2}$ & \multicolumn{2}{|r|}{1.5} & basic duration immunity \\
\hline$y_{1}^{\prime}(0)$ & 0.077 & 0.011 & susceptible persons $(0-5)$ \\
\hline$y_{1}^{2}(0)$ & 0.718 & 0.034 & susceptible persons $(>5)$ \\
\hline$y_{2}^{\prime}(0)$ & 0.007 & $0.04 \mathrm{I}$ & infected persons $(0-5)$ \\
\hline$y_{2}^{2}(0)$ & 0.076 & 0.231 & infected persons $(>5)$ \\
\hline$y_{3}{ }^{\prime}(0)$ & 0.008 & 0.039 & immune persons (0-5) \\
\hline$y_{3}^{2}(0)$ & 0.115 & 0.644 & immune persons $(>5)$ \\
\hline
\end{tabular}




\subsubsection{Impact of Control Programs}

If the case is considered in which mosquitoes and parasites do not adapt to the use of insecticides and drugs, the new equilibrium can be calculated, given that constant levels of insecticides and/or drugs are used. Because the impact of both control programs is modelled in a similar manner, they have identical effects. The control program will lower the rate of infection as a result of rendering the mosquitoes and/or parasites less fit, and of the decrease in the percentage of infected persons. The percentage of immune persons will likewise decrease, resulting in an increase in the size of the fraction of susceptible humans.

The incidence of malaria will decrease in regions of low endemicity, as a consequence of the control programs (Figure 9.8a). In regions of high endemicity, an increase of malaria may occur if the control programs are not stringent enough, the effect being a steeper increase in susceptible humans (immune persons lose their immunity) relative to the decrease in the infection rate (Figure 9.8b).

As a result of the ability of vector and parasite to adapt to the control programs, their effectiveness decreases in such a manner that the new equilibria are located nearer to those obtaining in the absence of control programs. It might be expected that adaptation may eventually lead to higher incidence rates than those which occur in the absence of adaptation. A notable exception is the case of low doses in regions of high endemicity, since adaptation will then result in a less pronounced increase in susceptibles which will exceed the reduced decrease in the infection rate, leading to lower incidence rates. 

malaria

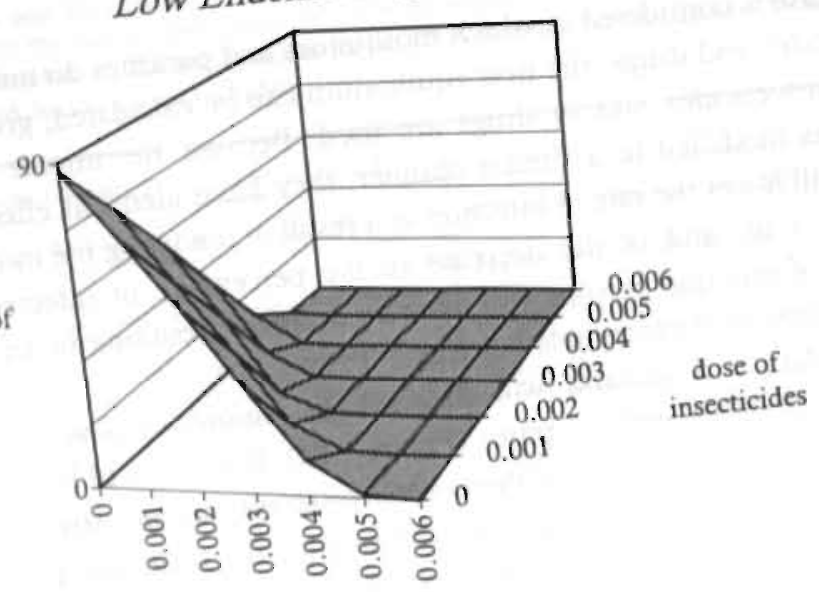

dose of drugs

High Endemic Region

incidence of malaria

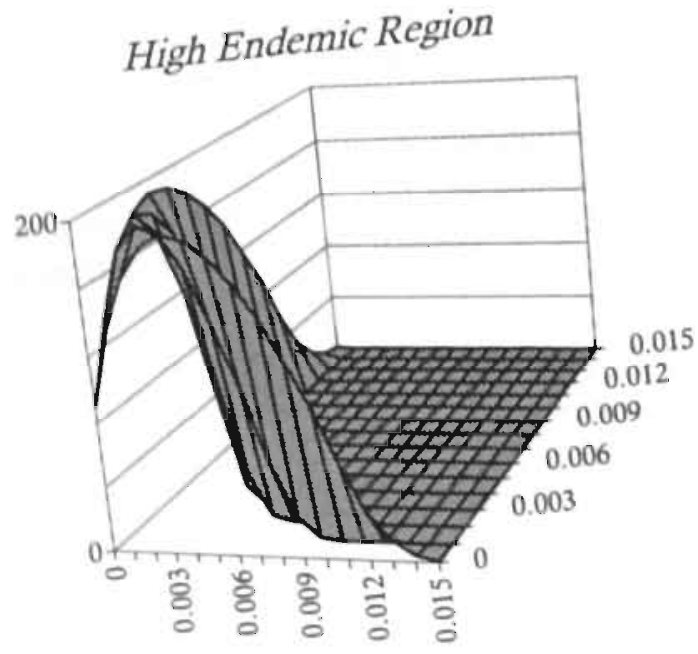

dose drugs

Figure 9 incidence of malaria for different levels of control in case of no adaption (high and low endemic regions) In Figures $9.9 \mathrm{a} \& \mathrm{~b}$ the averaged value endemicity, the vectorial capacity
control programs. In regions of low to a level
but due to adaptation among mosquitoes subsequently increases, albeit to 
which lies somewhat below the initial level. The result is a similar pattern in the incidence of malaria, although the level continues to fall (gradually). It is, thus, evident that a combination of both drugs and insecticides at low levels is more efficient than high level use of only one of the two, a finding which reflects the enhanced development of resistance at higher doses.

In regions of high endemicity, the decrease in vectorial capacity exhibits a similar pattern to that which occurs in regions of low endemicity. It would be expected that resistance development would differ in the two regions, due to a difference in the gene pool. Nevertheless, for simplicity's sake, the same fixed population size is used within the genetic algorithm and, therefore, arrived at similar results. An improvement of the model might be the coupling of vectorial capacity and the population size of the genetic algorithm. Due to the difference in the profiles of the populations, the patterns of incidence of malaria would be quite dissimilar. Following a reduction in incidence at the outset of the control programs, incidence subsequently shows an increase due to the lower effectivity of the control measures. Due to the high fraction of susceptible humans after a successful period of control, again as a result of the flow of immune persons due to the increased rate of immunity loss, incidence may even rise to surpass the initial level. In the long-run, a combination of too low levels of control does not achieve a better performance than control by' a single method. Indeed, incidence peaks at a level even higher than the initial (pre-control) level due to the higher number of susceptible humans who become reinfected.
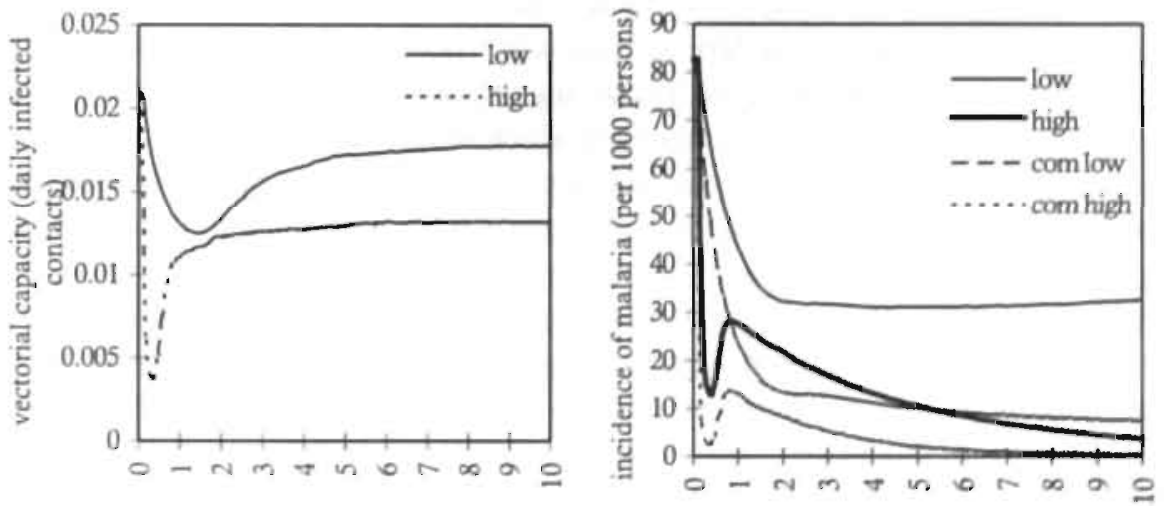

Figure 9.9a: Vectorial capacity and incidence of malaria for different levels of control in a region of low endemicity. Scenarios low and high depict the results of a low or a high dose of insecticides. For the scenario com low, low doses of insecticides as well as drugs are combined. This also holds for com high. 

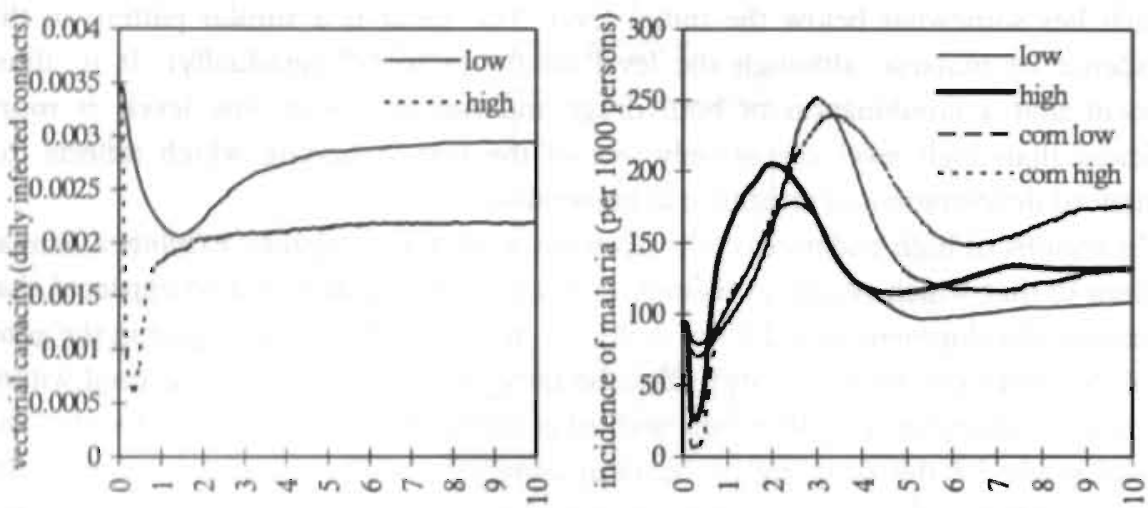

Figure 9.9b: Vectorial capacity and incidence of malaria for different levels of control in a region of high endemicity. Scenarios low and high depict the results of a low or a high dose of insecticides. For the scenario com low, low doses of insecticdes as well as drugs are combined. This also holds for com high.

\subsubsection{Sensitivity of Malaria Incidence to Migration}

Migration of mosquitoes and parasites can influence the development of resistance. Comins (1977), for example, showed that the migration of insects may greatly retard the development of insecticide resistance, and recent observations in Papua New Guinea and Tanzania support such model-based hypotheses (Paul et al., 1995). Various studies (e.g. Comins, 1977; Tabashnik and Croft, 1982) found two distinct phases in the time required to develop resistance. At low doses, resistance developed more rapidly as the dose increased, paralleling the case in which migration is absent, this in contrast to the case of high doses in which resistance develops more slowly as the dose increases. In the absence of migration, the rate of resistance development is determined primarily by the rate at which susceptible genes are removed from the population. As the dose increases, susceptible genes are removed more rapidly, and resistance consequently develops apace. At low doses in the presence of migration, the pattern is similar. Where migration is present and doses are high enough to kill heterozygotes, (which are intermediate between the susceptible and resistant genes, comparable with moderately-resistant in this Chapter), however, mosquito mortality due to insecticides also removes resistant genes from the population. As dose increases in this range, more heterozygotes are killed, leaving relatively few resistant mosquitoes. The resistant survivors are effectively swamped by the susceptible immigrants, thereby retarding resistance development.

The impact of mosquito migration on insecticide resistance development is analyzed by postulating various levels of insecticide application and various 
percentages of migration and subsequently calculating the number of time steps required for $50 \%$ of the genes to achieve resistance. The results are depicted in Figure 9.10 and show, as expected, that the migration of susceptible mosquitoes impedes the development of resistance. Furthermore, at high levels of migration ( $>40 \%$ inflow of susceptible mosquitoes), the development of insecticides resistance among the mosquitoes will be entirely blocked.

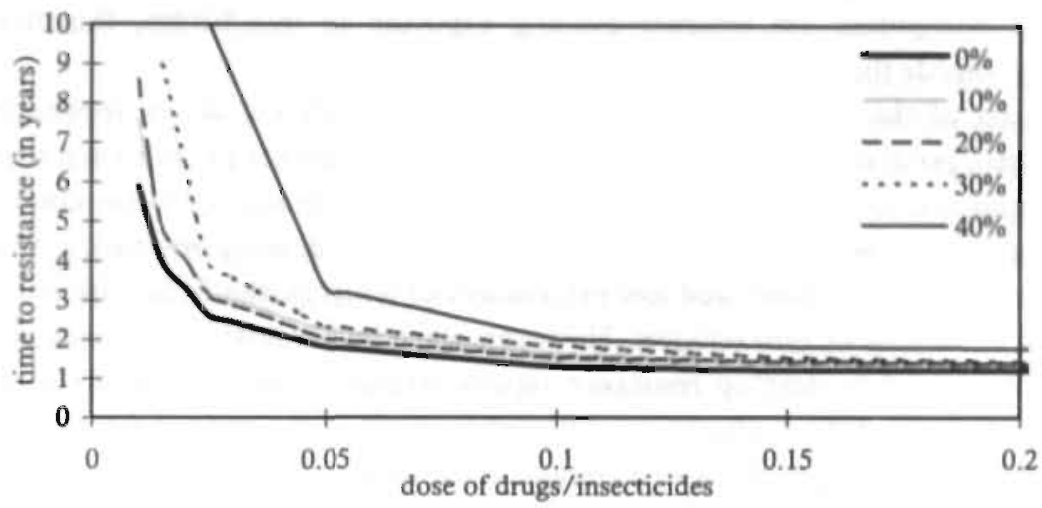

Figure 9.10: Effect of dose on the rate of evolution of resistance featuring various percentages of migrants per time-step.

That the results do not show the two distinct phases which were found in Comins (1977) and Tabashnik and Croft (1982) is a consequence of the different fitness function $^{5}$ for the various genes. The relative fitness among the various gene combinations remains rather the same, along the line of increasing doses of insecticides. This is not the case where models, such as the one adopted by Tabashnik and Croft (1982), are concemed, since heterozygotes are not killed at low doses, but only at high ones. In fact, in such models, there is a kind of threshold value in the fitness function (survival rates for the different types of genes), while in the model, a more gradual decrease of the fitness function is assumed. There was no field data. known at the time of writing which would favor either of these approaches.

\footnotetext{
${ }^{5}$ Comins (1977) and Tabashnik and Croft (1982) do not actually employ the term "fitness function", but I use an interpretation that is equivalent to their "dose-mortality lines".
} 


\subsubsection{Sensitivity of Malaria Incidence to the Coverage Rate}

In the absence of refugees from control programs (i.e. $100 \%$ coverage), rates of insecticide resistance increase with increasing doses. If, however, a fraction of the mosquito population evades treatment by becoming "refugees", the development of resistance is expected to be impeded. Tabashnik (1990), for example, shows that if $10 \%$ of the mosquitoes are refugees evading exposure to insecticides, this may significantly impede the development of resistance.

The impact of the coverage rate is explored for the different doses applied in various control programs, and the results are depicted in Figure 9.11. For each time step, a certain fraction of the mosquito population is not reached by the control measures, and two distinct phases in the time required to develop resistance were found. In the case of low doses and low percentages of refugees, the results are about the same as in the case of zero refugees. However, when higher doses are applied, the time period required to develop resistance rapidly lengthens. The doses of control which mark the two distinct phases are different for each of the various fractions of refugees. Where higher percentages of refugees are concerned, the period of time required to develop resistance starts to become greater at an earlier juncture. Among more than $50 \%$ of the refugees, resistance will not develop at all. The rate of evolution of resistance by $P$. falciparum could be retarded by selective treatment of those people with high parasitaemias.

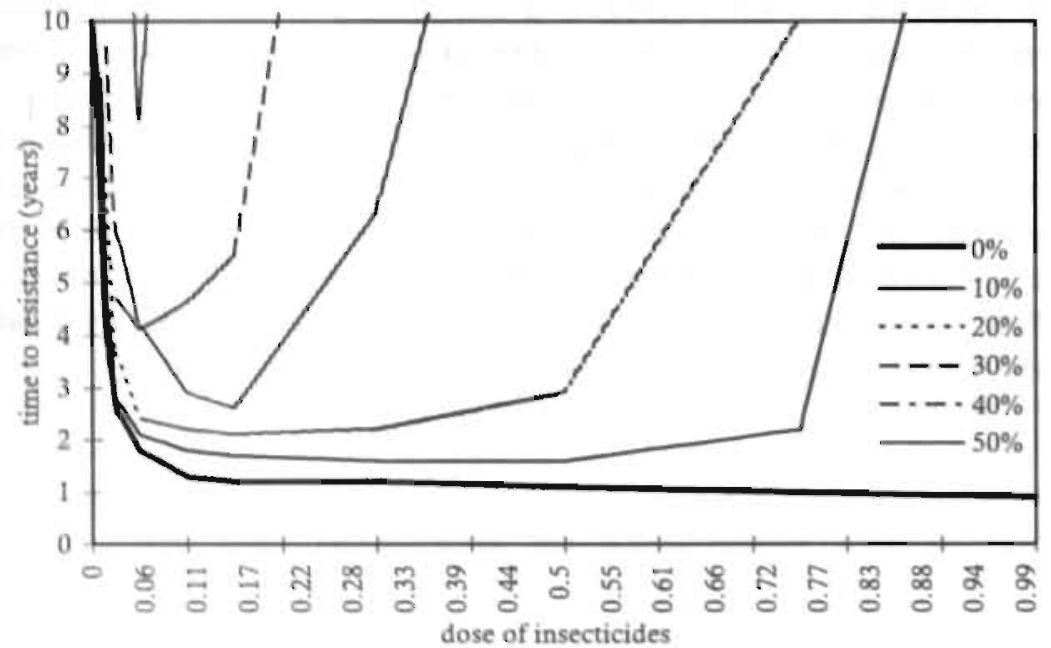

Figure 9.11: Effects of dose on the rate of evolution of resistance featuring various percentages. of refugees per time-step. 
An explanation for the existence of these two distinct phases, which are also found by Tabashnik (1990), is the fact that during the period in which the mosquito evades treatment, the benefits of being resistant do not hold. In other words, mosquitoes will not benefit from being resistant in periods during which they are not being sprayed with insecticides. On the contrary, during such periods, susceptible mosquitoes enjoy a higher biotic fitness than resistant mosquitoes. By the same token, in the periods during which the mosquito population is reached by insecticides, a resistant mosquito enjoys the benefits of higher fitness. In the case of higher doses, the difference in fitness in the two cases (reached or not reached by a control program) becomes greater, resulting in the time required to develop resistance becoming longer. Furthermore, the presence of a higher fraction of refugees decreases the average time during which the population, in general, profits from the availability of resistant genes, consequently impeding the development of resistance.

\subsubsection{Sensitivity of Malaria Incidence to Temperature Change}

In this subsection, the impact of increasing temperature levels is analyzed. For both the low and the high endemic regions, the change in the occurrence of malaria is simulated in the event of a temperature increase of 0.25 or $0.5{ }^{\circ} \mathrm{C}$ within a decade (Figure 9.12). This temperature increase lies within the range projected by the IPCC (1991).

The impact of temperature change on the incidence of malaria would be significant. The vectorial capacity would increase, due to increasing biting rates and shorter incubation periods, which would lead to an increase of the incidence of malaria in the order of $50 \%$ to $100 \%$ in regions of low endemicity. In regions of high endemicity, the incidence of malaria would fail by about 15 to $30 \%$ in the event of such temperature changes, as a result of the increase in the collective immunity.

The optimal temperature for mosquito survival would increase by $0.05^{\circ} \mathrm{C}$ in areas of low endemicity and $0.12^{\circ} \mathrm{C}$ in highly endemic areas, a development which lags behind the local mean temperature increase. The impact of mosquito adaptation to temperature change compared with no adaptation is not significant. This is caused by the fact that only the life expectancy changes as a. result of adaptation, and this is only one of the factors (besides the man-biting habit and the incubation period) which are related to temperature fluctuations. 


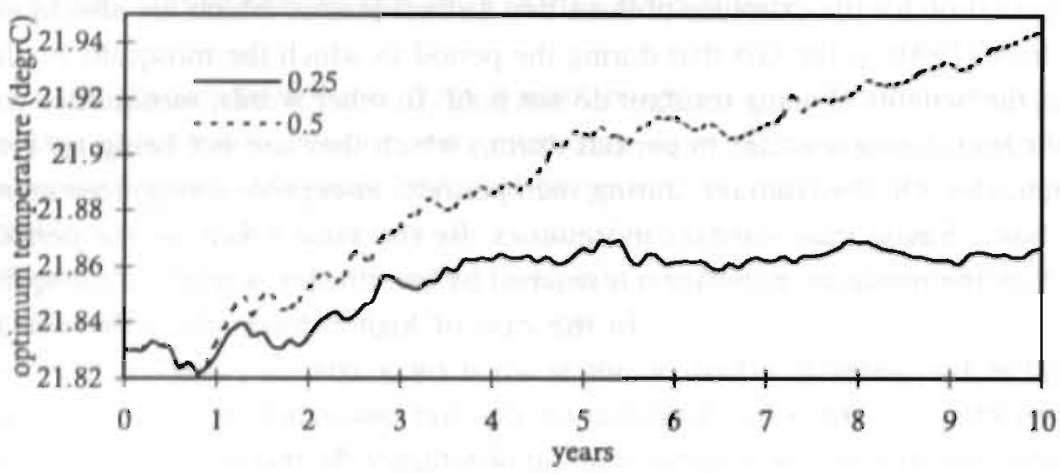

Figure 9.12a: Optimum temperature given a 0.25 and a 0.5 degreee Celsius temperature increase.

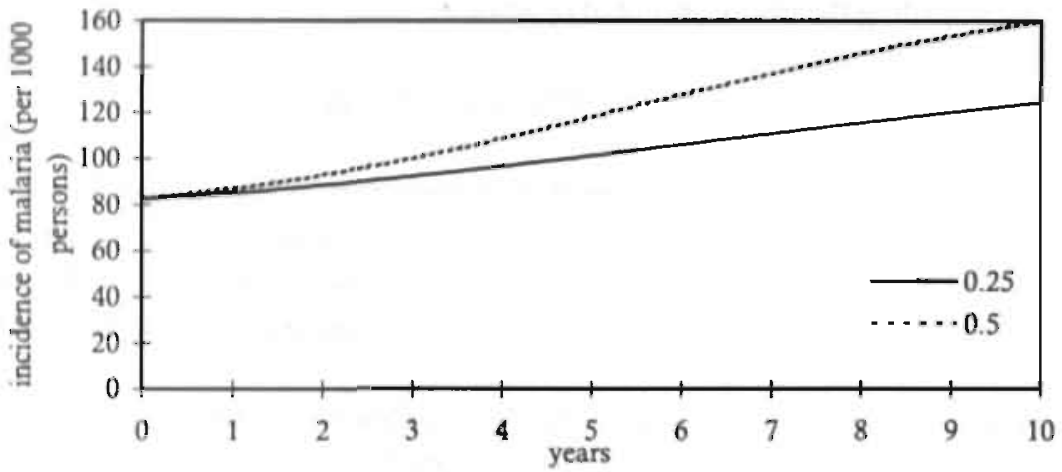

Figure 9.12b: Incidence of malaria given a low endemic region for a 0.25 and a 0.5 degree Celsius temperature increase.

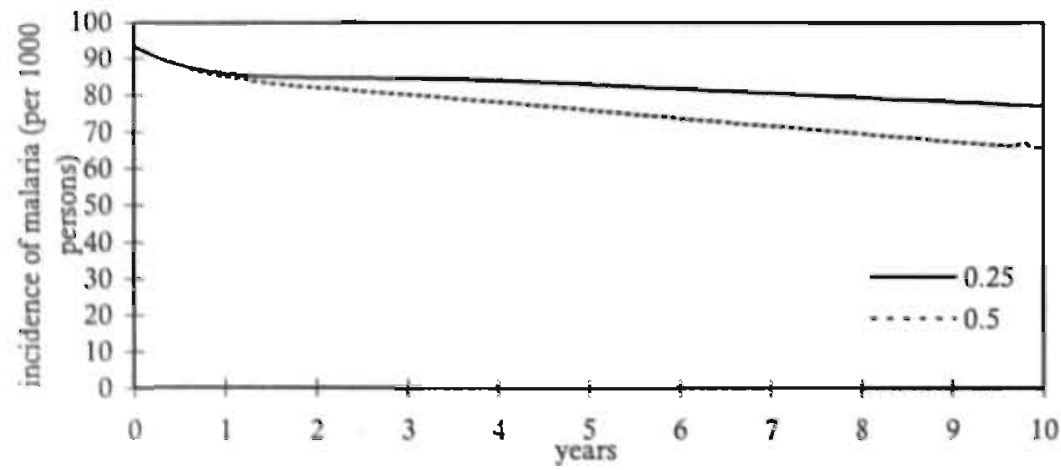

Figure 9.12c: Incidence of malaria given a high endemic region for a 0.24 and a. 0.5 degree Celsius. temperature increase. 
An important factor influencing the rate of the evolution of resistance is the number of generations per year: resistance develops faster as the annual number of generations increases. Increasing temperatures will lead to a more rapid succession of generations per of unit time (Jetten and Takken, 1994). As a consequence, resistance development in the malaria mosquito population as presented in this Chapter would have taken place within a shorter time span, if a temperature-dependent generation time was implemented.

\subsubsection{Sensitivity of Malaria Incidence to Initial Resistance}

The sensitivity of the initial fraction of resistant mosquitoes and parasites was investigated by an illustrative experiment. A low and a high dose of insecticides is postulated in a highly endemic region. Figure 9.13 shows that a higher level of initial resistance leads to a more rapid penetration of resistant genes in the mosquito population. Because of a higher selection pressure on the population, the time required to develop resistance decreases in an environment of higher doses. This finding corresponds with those reported in Tabashnik (1990).

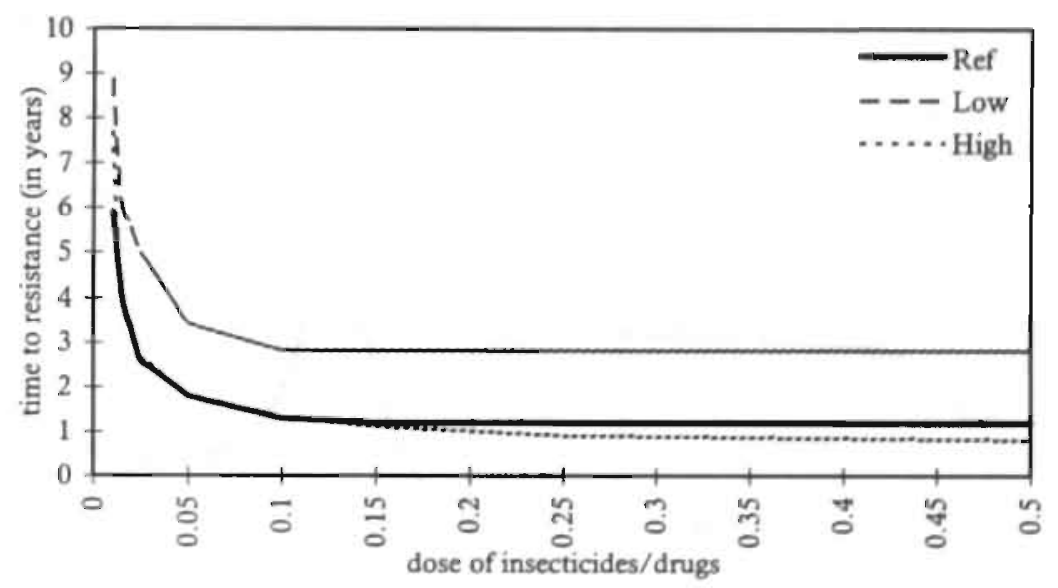

Figure 9.13: Time to resistance for different levels of initial resistance, where low represents $0.0 \%$ initial resistant genes, high represents $1 \%$ initial resistant genes, while the reference is $0.1 \%$ initial resistant genes. 


\subsubsection{Sensitivity of Malaria Incidence to Operators of the Genetic Algorithm}

Within the range of reasonable values of the main operators of the genetic algorithm, a number of values are selected to test the sensitivity of the solutions vis-àvis the chosen values. As depicted in Figures $9.14 \mathrm{a} \& \mathrm{~b}$, the results do not seem to be sensitive to the chosen values, although some variation does occur. This supports the chosen (unknown) values of the operations.
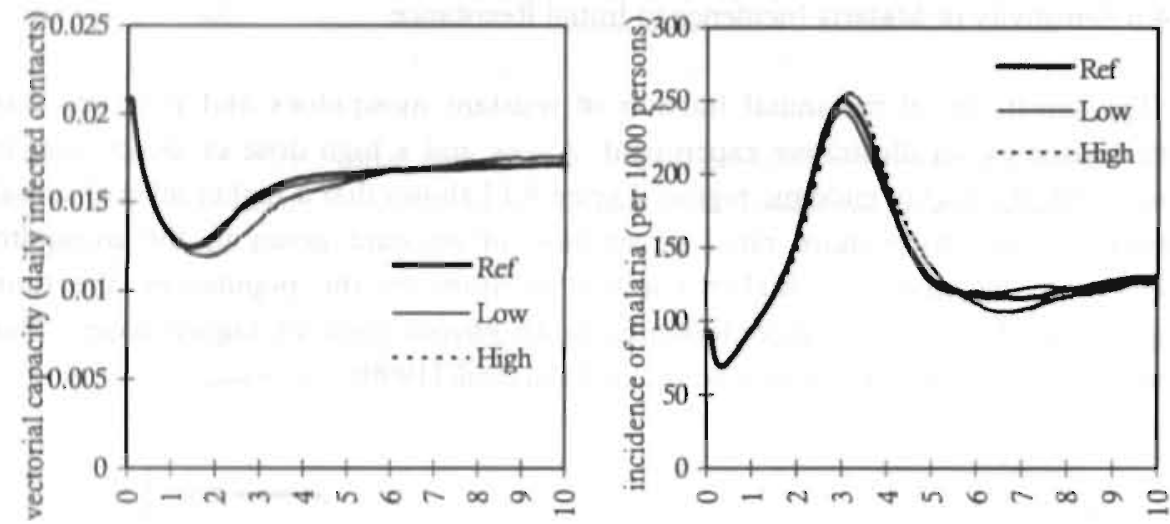

Figure 9.14a: Vectorial capacity and incidence of malaria for different levels of crossover probability, where low represents 0.2 , high 0.8 and reference a probability 0.4 .
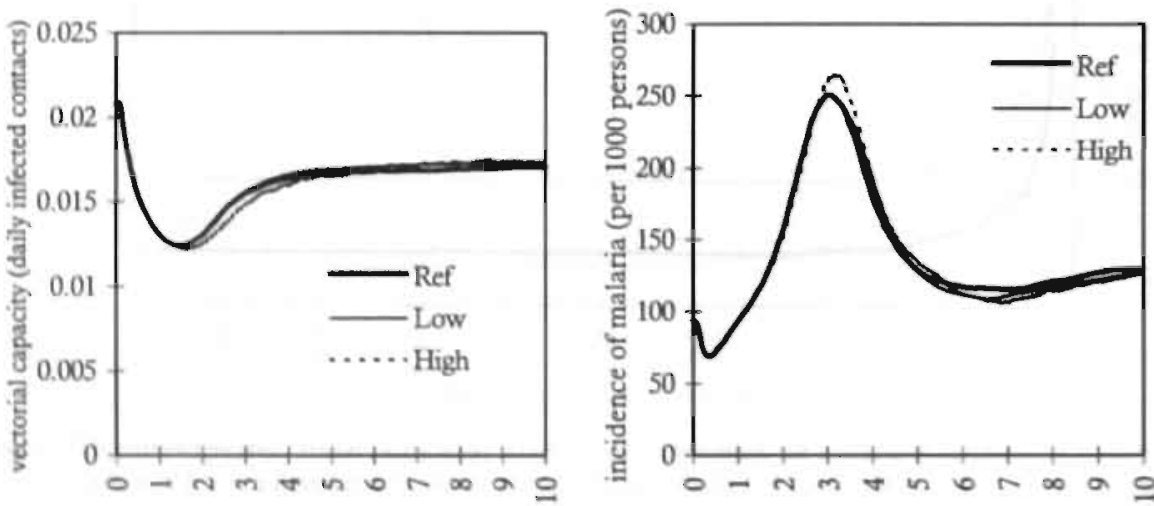

Figure 9.14b: Vectorial capacity and incidence of malania for different levels of the mutation probability, where low represente 0.0001 , high 0.01 and reference a probability of 0.001 . 


\subsubsection{Adaptive Malaria Management}

In this subsection, the impact of the combined effects of climate change and resistance development on the prevalence of malaria are analyzed among mosquitoes and parasites. This analysis is performed using an adaptive management style, i.e, one which relates the level of control programs according to the observed state of the system. Since, in the model the resistance development dynamics are implemented in an identical manner for both mosquitoes and parasites, only one of the two needs to be considered in the analysis, and the mosquitoes are selected for this purpose.

The use of insecticides is related to the observed incidence of malaria, and there are two levels of application: a zero dose and a high dose. If the incidence of malaria fell below 20 per 1000 persons, the use of insecticides is assumed to be stopped, while if malaria once more exceeded this level, it would be reintroduced again (at high dose levels). Furthermore, if the incidence of malaria exceeded the level of 100 per 1000 persons, which is above the initial level, the use of insecticides would be stopped as not being effective.

The results set out in Figure 9.15 illustrate that in areas of low endemicity, the use of insecticides leads to a successful control of malaria occurrence. However, if the temperature was to increase by some $0.5^{\circ} \mathrm{C}$ within a single decade, the efforts to control malaria would have to be intensified significantly. In areas of high endemicity, the control of malaria fluctuates during the decade, while the incidence would continue to fluctuate around the level of 100 per 1000 persons, regardless of any temperature increase. 
Meetino Targets
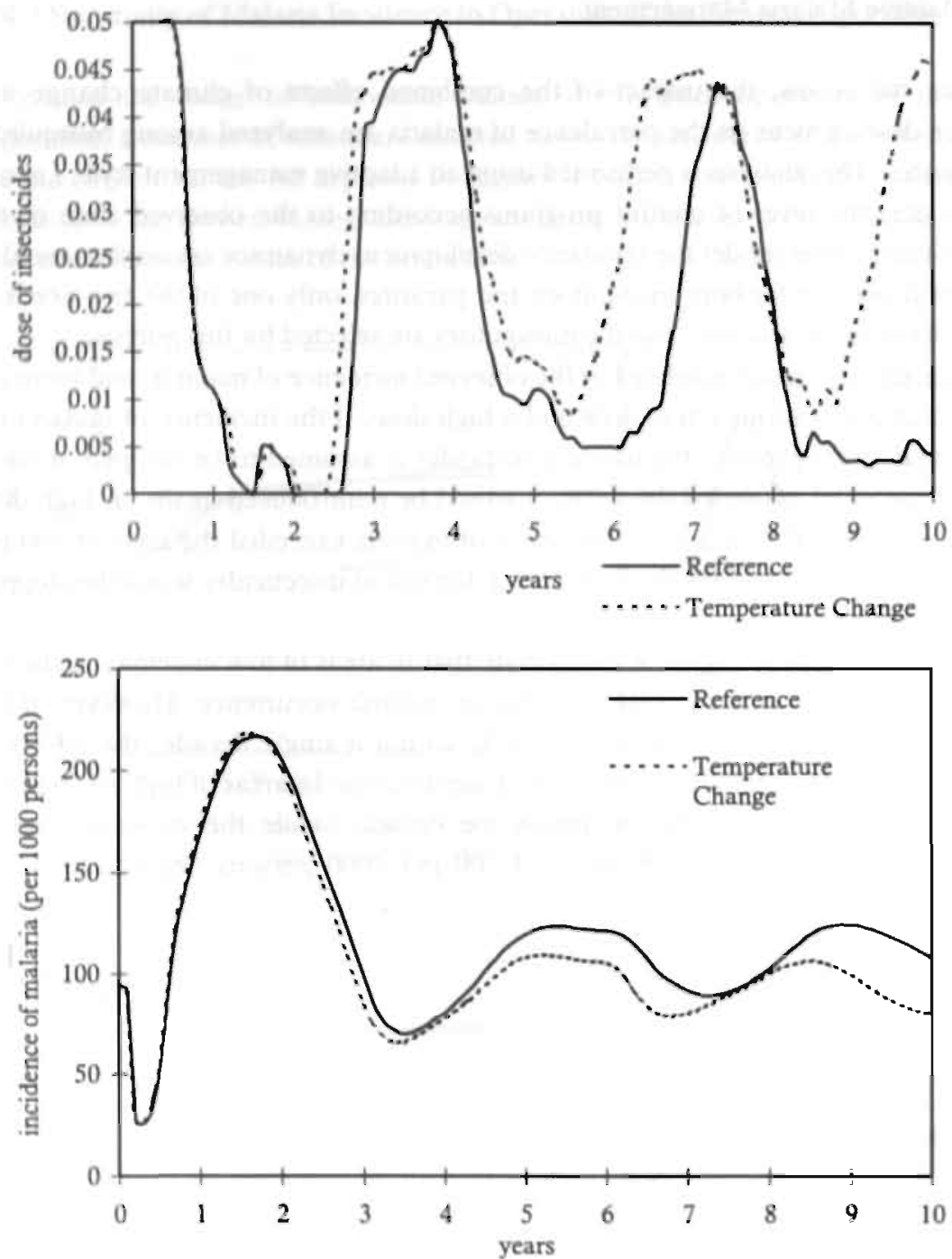

Figure 9.15a: Control patterns and malaria incidence for a region of high endemicity in the event of an adaptive management style if being adopted. The different lines show the impact of a projected climate change of the adaptive control programs. 

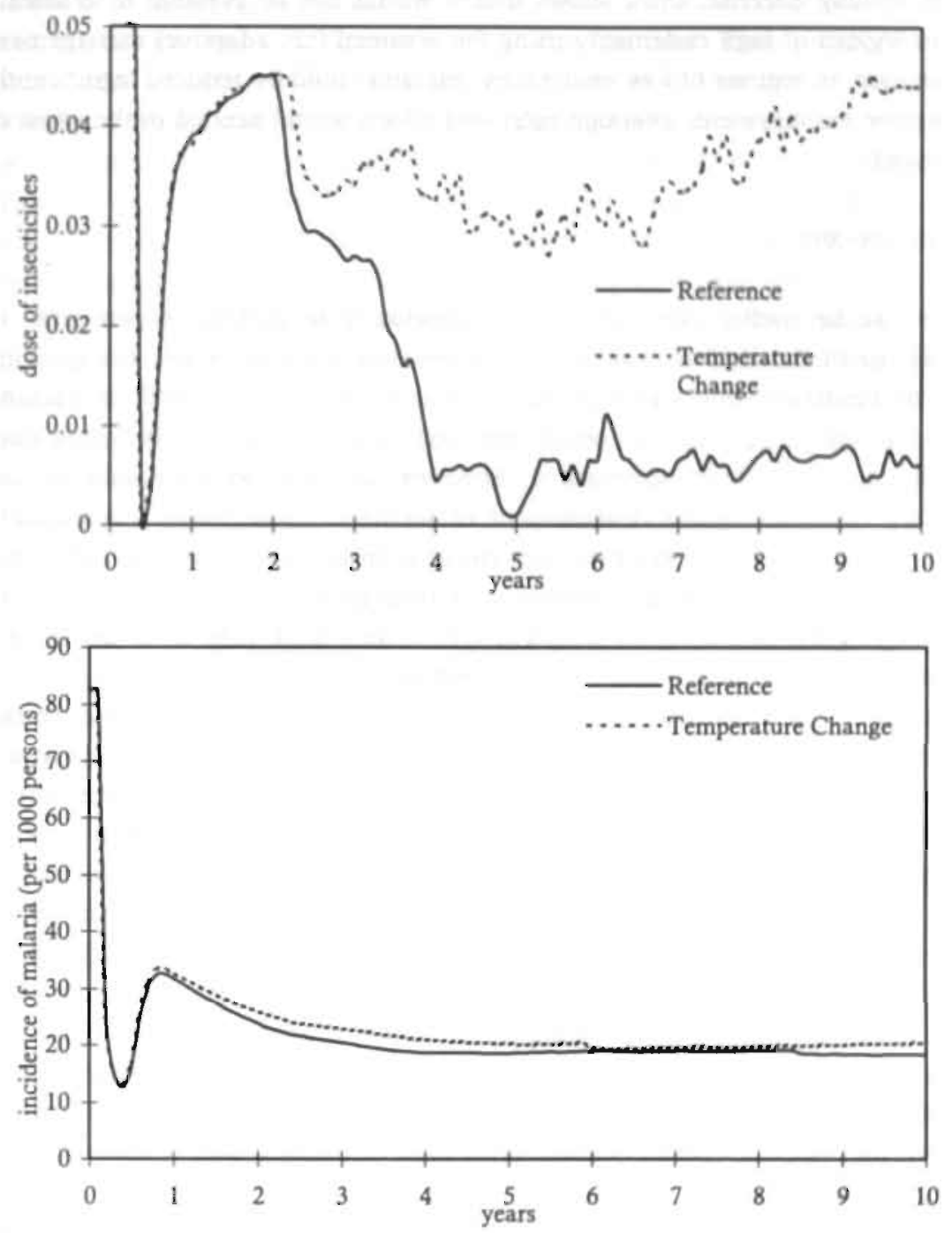

Figure 9.15b: Control patterns and malaria incidence for a region of low endernicity in the event of an adaptive management style is being adopted. The different lines show the impact of a projected climate change on the adaptive programs. 
This modelling exercise, thus, shows that it would not be possible to eradicate malaria in regions of high endemicity using the assumed (i.e. adaptive) management style. However, in regions of low endemicity, malaria could be reduced significantly using adaptive management, although increased efforts would needed in the event of climate change.

\subsection{Conclusions}

Models can be useful, especially if the opportunity to perform experiments in laboratories or in the field is limited. This is certainly the case where the growing problem of resistance development among malaria vectors, as well as malaria parasites, to control programs is concerned, and much remains to be elucidated. Current malaria modelling approaches, however, do not explicitly address the evolutionary character of the development of resistance. The malaria assessment model presented in this Chapter is neither comprehensive nor predictive, but rather intended to include evolutionary processes of resistance development in order to provide insights into this complex adaptive system and, thus, help us to arrive at a better understanding of the possible effects of control programs.

The results suggest that adequate use of insecticides and drugs may reduce the occurrence of malaria in regions of low endemicity, although increased efforts would be necessary in the event of a climate change. However, the model indicates that in regions of high endemicity, the use of insecticides and drugs may lead to an increase in incidence, due to enhanced resistance development. Projected climate change, on the other hand, may lead to a limited reduction of the occurrence of malaria due to the presence of a higher percentage of immune persons in the older age class. Elements of a sustainable antimalarial policy in regions of high endemicity will probably need to rely upon a stimulation of socio- economic development and provision of vector-proof housing. However, given the multiplicity of ecological and biological element and of the natural, adaptive defense mechanisms of the malaria parasite/vector complex, control or eradication must be planned with consideration of prevailing local conditions.

The present version of the model simulates the incidence of malaria based on the use of insecticides and medication, together with temperature change. Additional factors would need to be included before one could speak of an integrated approach to the malaria problem, whereby the inclusion of environmental management would be of particular importance. The effects of land use changes, water management, housing, etc., on vectorial capacity would, therefore, need to be incorporated. As a means of accommodating such spatial differentiations, the use of cellular automata 272 
might be considered. A cellular automaton consists of an array of cells in which each cell can assume one of $\mathrm{k}$ discrete states at any one time. Time progresses in discrete steps, and all cells change state simultaneously as a function of their own state, together with the state of the cell in their neighborhood, in accordance with a specified set of transition rules (e.g. Gardner, 1970; Tobler, 1979; White and Engelen, 1994; Engelen et al., 1994). In fact, the present model simulates the dynamics of a single cell, whereas in a spatial model it would be connected to other cell by, for example, incorporating migration of mosquitoes and the impact of land use changes.

The modelling approach presented here fits well in the qualitative attention currently being paid to the importance of evolutionary principles (e.g. Levy, 1992; Ewald, 1994). However, a great deal of empirical research is needed to improve the modelling approach. In the specific case of malaria, it is especially important that more insights into the possible shapes of the fitness functions of the parasites and the mosquitoes are acquired. This need is illustrated by the results on the impact of migration on the development of resistance at high doses, since they differ from the results of previous studies as a result of different assumptions regarding the fitness functions. Nevertheless, the fact remains that development of integrated assessment models which are based on the evolutionary and local dynamics of ecological systems may prove essential to assessing future developments in these complex adaptive systems. 


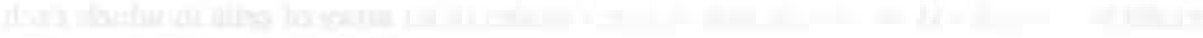

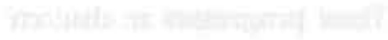

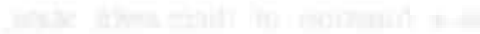

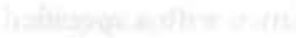

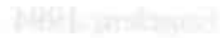

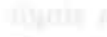

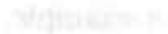




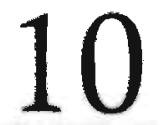

Conclusions and Discussion

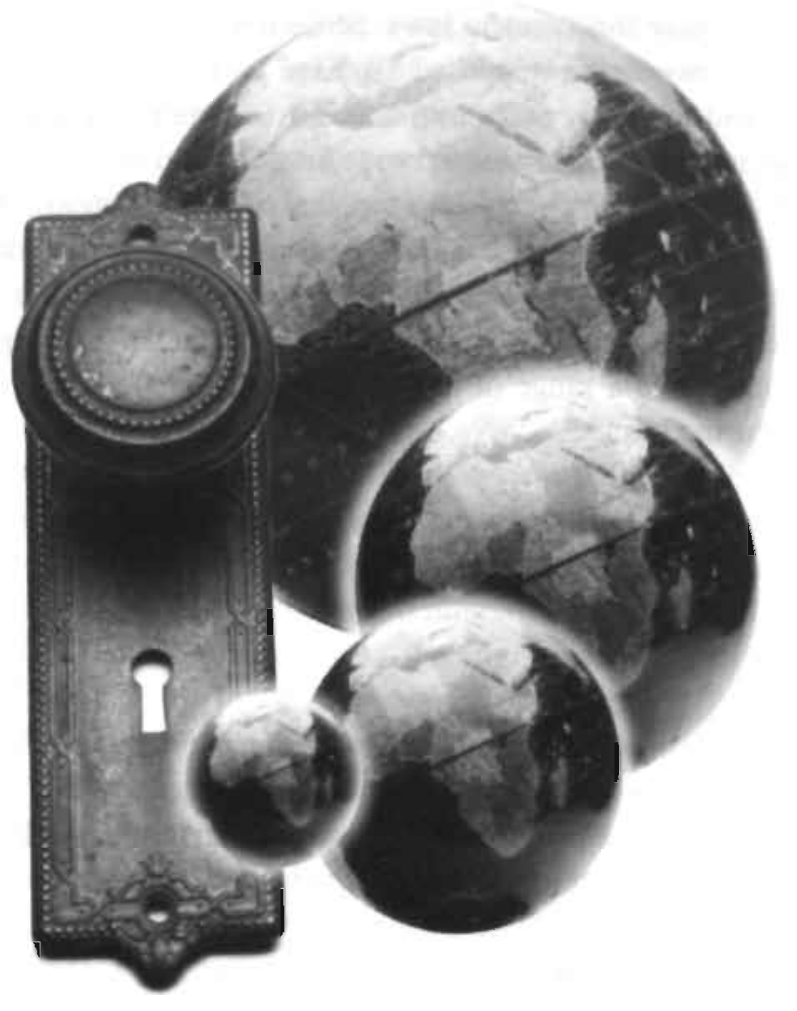


As I was originally trained in econometrics and Operations Research, the membership of the multi- and interdisciplinary research program "Global. Dynamics and Sustainable Development" made me aware of the different approaches in modelling and uncertainty analysis. After having read "Cosmopolis" (Toulmin, 1991), in which Stephen Toulmin describes the rise and fall of modernism, I recognized that the integrated assessment modelling approach is an element in the transition of modernism to post-modernism. Modernism is characterized by its rational search for universal time-and place independent laws. Science is meant to search for certainty. Post-modernism, however, is supposed to have a more practical attitude (Van Peursen, 1994), and explicitly takes into account uncertainty and local knowledge. With respect to integrated assessment modelling, its aim is to integrate scientific "state-of-the-art" information for practical policy-related problems. However, as I address in this dissertation, the various traditional mathematical paradigms employed by the different research groups preclude the research society with a real integrated modelling paradigm. In this dissertation, various methodological improvements are suggested and applied to a range of topics related to global change to improve the practical use of the models.

\subsection{Major Findings}

\section{Methodology}

\section{Optimization}

Optimization aims at determining the decisions to derive the best performance of a system under concern. To date, various optimization oriented models exist for climate change policy and environmental policy in general. One of the characteristics of the current generation of optimization models is that they depend upon the restrictive requirements of continuity and derivative existence. However, the awareness of the unpredictable nonlinear behavior of the global system leads to discontinue and noisy search spaces, which are not to be dealt with with traditional optimization tools. The systems can, thus, be linearized and simplified, in order to use the traditional optimization techniques, or, alternatively, new search routines can be applied to advanced complex simulation models.

In this dissertation, two optimization case studies are discussed to investigate the latter approach. A mathematical system is constructed which combines the economics of DICE and the climate dynamics of the mathematical system version of IMAGE 1.0. Various traditional and new optimization routines are used to solve a set of optimization problems. This model, OMEGA, is used to analyze the impact of an 
inadequate representation of the dynamics of the climate system as reasonably understood by natural science in current optimization models for climate change. Because a parameterized model (like DICE) is fully calibrated on historical data, and explains, therefore, only a limited range of the system, a process-oriented model (like OMEGA), however, describes a larger range of the system behavior. Compared with DICE, OMEGA leads to an enhanced effort in reducing emissions for cost-benefit analysis. In the event of severe constraints on concentration levels or temperature change, far fewer emission reductions have to be made, if OMEGA instead of DICE is used. These differences could be explained by the different descriptions of the dynamics of the climate system.

In a second study, exploratory experiments with the energy-cycles part of TARGETS have been investigated. A genetic algorithm in which a set of solutions competes for "survival", is used to solve the optimization problems. The algorithm is used as a supporting tool for the search for specific scenarios and is supposed to be a helpful tool in scenario analysis of TARGETS(-like) integrated assessment models.

\section{Perspectives}

Our understanding of the global Earth system is incomplete, and subjective judgments and disagreement originating from different interpretations of the blind spots can largely explain uncertainties in the images of the present and the future. I propose to capture uncertainties arising from subjective judgment and disagreement in the inclusion of various perspectives. To operationalize a perspective-based uncertainty analysis, I used the framework of Cultural Theory, although I acknowledge the weaknesses and deficiencies of the Cultural Theory. Drawing on the Cultural Theory, the perspectives are labelled "individualist", "hierarchist", and "egalitarian", and are represented by distributions of preferred values of parameters in the models of two case studies on allocating $\mathrm{CO}_{2}$ emission rights and projecting emission reductions of $\mathrm{CO}_{2}$. Including perspectives in the uncertainty analysis helps to explain and understand the various possible images of the futures and the various ways to respond. In case of the emission right allocation, it makes clear that the difference between possible initial allocations differ so much between the perspectives that it will be hard to find a compromise in implementing a tradeable emission right regime. In the case of the "Battle of Perspectives", it illustrates various alternative response scenarios assuming different world views. 


\section{Evolutionary Approach}

The present generation of integration assessment models is mainly based on the mechanistic paradigm. The Earth system is thought of as a machine which should be rationally controlled to reduce the pressures which could lead to instabilities of the "natural" equilibrium. The evolutionary approach rejects the equilibrium approach. From a mechanistic perspective, risks may increase if the system changes, while from an evolutionary perspective, risks may increase if the system is less able to adapt to a change.

The last decades, new evolutionary based approaches have been developed in studying complex adaptive systems: systems which organize their component parts to adapt themselves to the problems posed by their surroundings. This has provided the material for developing an integrated modelling paradigm in which I distinguish three different, but interwoven, levels of modelling reality. At the first level, the physical stocks and flows which can be simulated by sets of differential equations; a mechanistic approach. At the next level, the behavioral and informational structures of ecological, economic and social processes which can be simulated by evolutionary modelling: an organismic approach. At the third levels, the values and beliefs which might be simulated by adopting various (cultural) perspectives.

I performed two case studies in which evolutionary modelling has been applied. The first case study, the "Battle of Perspectives", shows that an evolutionary approach enables us to include learning and adaptive behavior in a simple climateeconomy framework. In contrast with the usually employed assumption of a rational agent with (near) perfect knowledge, a heterogenous set of agents respond to the observations. Due to the stochastic characteristics of the evolutionary processes, large uncertainty ranges are generated which derive from the unpredictability of the response of agents to the information from the system.

In the second case study, malaria is modelled as a complex adaptive system. The evolutionary modelling approach for malaria fits well in the present qualitative notice of the importance of evolutionary principles for infectious diseases. As in the "Battle of Perspectives", a genetic algorithm was included into a system dynamics model to simulate adaptive behavior, here of mosquitoes and parasites. Although the required data were not found to validate the model, experiments gave results conform current insights of experts. Furthermore, possible consequences of a climate change were analyzed. 
Applications

Energy-Climate

Exploratory experiments have been performed with the energy-cycles part of TARGETS. From an environmental perspective, a low fossil fuel energy supply is desired. However, what kind of a transition leads to low economic costs, in order to meet the growing energy demands in a sustainable way? Based on earlier studies, a set of scenarios is constructed in which different assumptions on technological development have been implemented. The different projections in costs and effects lead to the question what to do in the short term, given the important uncertainties in the longer term, and given the ambition to meet international climate change targets. The results of hedging strategies and delayed response scenarios suggest that a short term efficiency improvement due to an active energy policy is recommended, irrespective of the technological developments. Furthermore, if a transition to a low energy supply does not get initiated, there will be a risk of becoming locked in by the use of fossil fuels. This is due to the inertia of the energy system and the short term cooling effect sulphate aerosols.

Although a reduction of $\mathrm{SO}_{2}$ emissions is not recommended in meeting temperature change targets, it helps to reduce the problem of acidification. The move from a $\mathrm{CO}_{2}$ dominating debate to a $\mathrm{CO}_{2}-\mathrm{SO}_{2}$ dominating debate underscores the importance of an integrated assessment of the energy/environment system in which this study serves as a first modest step.

\section{Emission Rights}

The industrialized countries have so far caused the major part of $\mathrm{CO}_{2}$ emissions. From an egalitarian perspective, the industrialized world should take prime responsibility in responding and supporting the developing countries in contributing their share to mitigate climate change without thwarting their development. A reduction of the risk of adverse climate change according to the "precautionary principle" approach implies that future global emissions of greenhouse gases are constrained. Together with past emissions, a global carbon budget can be estimated, and be allocated among the population. If all world citizens living in the period 18002100 were allocated an equal emission quotum, this would result in a per capita emission of about $1 \mathrm{tC} /$ cap yearly, assuming a future scenario according to IS92C (= a global budget of $1130 \mathrm{GtC}$ ). The industrial regions would have exceeded their allocated budgets for 1800-1990, by about $90 \mathrm{GtC}$ (OECD) and $20 \mathrm{GtC}$ (CIS). The developing countries, however, have an emission credit of about $130 \mathrm{GtC}$. 
A more sophisticated allocation model is used, which takes into account historical and future development for the criteria: population size, GNP, and energy use. There are various perspectives pertaining to the allocation of a constrained global fossil $\mathrm{CO}_{2}$ emission budget. In the first place, there might be differences in the scenarios concerning fossil $\mathrm{CO}_{2}$ emissions, energy use, population, technology, and economic development. Second, the preferred value distribution of parameters of the model might be skewed by perspectives. Because of the political dimension of the subject and the dynamic shifting of the mixture of perspectives in time, the results show that there is no clear initial allocation of emission rights.

\section{Battle of Perspectives}

The concept of the "Battle of Perspectives" is meant to illustrate a possible incorporation of agent behavior within integrated assessment models. Agents' perspectives which determine their management style can change, if they are confronted with a persistent pattern of surprises. Although there is no claim for reality, exploratory experiments suggest that in case of successful adaptation, alternative images of the future can be generated. Futures featuring high levels of fossil $\mathrm{CO}_{2}$ emissions are associated with low temperature increases, as well as low emission paths together with high temperature projections. In a speculative way, the impact of possible surprises can be analyzed, which is performed for a so-called cooling surprise. Suppose temperature increase is masked by a cooling effect; this leads to a later reduction of emissions and a higher temperature increase compared with the case we had the valid observations. So, even if agents were modelled as being adaptive, the everlasting incomplete knowledge of the system makes it possible to miss the policy targets.

\section{Malaria}

Results suggest that adequate use of insecticides and drugs may reduce the pccurrence of malaria in low endemic regions, although increased efforts are necessary in case of a climate change. However, in high endemic regions, the model indicates that the use of insecticides and drugs may lead to an increase of the incidence due to resistance development. A projected climate change, however, may lead to a limited reduction of the occurrence of malaria, due to a higher percentage of immune persons in the older age class. A "sustainable" antimalarial policy in high endemic regions will probably be found in a stimulation of socio-economic development and vector-proof housing.

Migration of (susceptible) mosquitoes and parasites may retard the resistance development significantly. Together with the expected relevance of land use changes, 
a next step in developing an integrated assessment tool for malaria is the inclusion of spatial characteristics, based on, for example, cellular automata.

\subsection{Future Research Avenues}

As the reader probably may have noticed, this dissertation is a collection of ideas which are not all worked out in detail and which address a wide range of issues in the emerging field of integrated assessment modelling. Given the findings of this dissertation and my experience in integrated assessment modelling, I have tried to sketch a possible framework of integrated assessment modelling which might be more fruitful than present approaches. The aim of such a framework is that it serves as an integrated toolbox for a large range of topics related to global change and sustainable development. The models in this toolbox should be interactive, while the aim of the framework is to derive insights of the global system, i.e. making complex systems less complex. But avoiding making one model to fit all, the toolbox should have different types of models addressing different types of problems, dynamics, aggregation and time-scale. I propose to call this interactive toolbox for global change TOPICS, standing for TOolbox to support Policymaking for Integrated Complex Systems. TOPICS may contain three different types of models: information systems, global dynamic models, and local dynamic models. These models are intensively related with each other. The choice for the different tools in the toolbox, is the different kind of dynamics (local and global) which may influence each other, but may not be included in one interactive model, and balance the scientific knowledge and the desire to support policy.

\section{Information Systems}

The available data is hardly sufficient to adequately characterize the processes being modelled. We should not aim at modelling the global system integrating all social, economic, and ecological processes in geographical detail, while these kind of models cannot be validated (Oreskes et al., 1994), are not suitable for interactive analysis, and may give a misleading image of our scientific knowledge.

However, we need geographical information systems to measure global changes and to identify areas at risk. Therefore, such an information system can serve as an intermediary between the rather abstract and hypothetical projections of the simulation models and the state of the global earth system. 


\section{Global Dynamic Models}

A regionalized world model can be made in which the dynamics of the regional population, economy, energy and mineral use, and the flows between the regions (migration, trade) are simulated, as well as the consequences of the disturbed global element cycles causing climate change, stratospheric ozone depletion, and depletion of resources.

Such a modelling framework enables us to perform target-oriented analysis, like Chapter 6, to identify possible trade-offs in policies and synergetic effects of the various topics. Regional differences in impacts of global change and possibilities to reduce the pressures will motivate regional strategies. What are "optimal" strategies for the regions themselves, and how will cooperation stimulate the ability of meeting global targets? Current efforts in this research domain are rather theoretical and the challenge will be to apply the concepts on more advanced simulation models to identify suitable (inter) regional strategies for meeting global targets.

\section{Local' Dynamic Models}

These kind of models are based on evolutionary modelling techniques and are. meant to derive insights in systems of change. The user may derive in such interactive game-oriented framework a less complex picture of complex systems. Two possible topics which may be addressed:

\section{* Social Dynamics}

If policy is meant to influence human activities, insights in the driving forces of behavioral change are required. As an aid to communicate social psychological insights to vanious disciplines and decision makers, and to explore possible behavional change for proposed policy actions, a toolbox might be helpful which simulates the basic concepts of behavioral change. In Jager et al. (1996a), a conceptual behavioral model is developed aimed at being used for integrated assessment modelling. Using the insights, derived from evolutionary modelling, a multi-agent model is under development which simulates the agents' decision processes based on motivations to use opportunities and having the abilities to meet their needs (Jager et al., 1996b). Compared with the "Sugarscape" model of Epstein and Axtell (1995), the model of Jager et al. (1996b) will be focused on including social psychological insights. Such a tool might communicate insights on possible behavioral changes due to the impacts from and the response to global change. 


\section{* Local Dynamics and Global Change}

Many local human activities intensify the pressure on the global system leading to a diversity of impacts on local scale. The aggregation of all these pressures and impacts can be considered as global change. The current generation of integrated assessment models primarily focuses on the macro dynamics of global change, which is mainly caused by the climate change oriented background of most of the current integrated assessment models. However, getting more grip on how to reduce the pressure and explore the possible impacts, an interesting alternative is to develop integrated assessment models for a typical area in which local dynamics and spatial diversity are included. An interesting example in this field is the ISLAND model of Engelen et al. (1994), which simulates the social and economic developments and their environmental consequences of a typical small island in the Caribbean. The ISLAND model includes the spatial characteristics of the problem, but does not include the local dynamics of the various important social and ecological systems. The malaria model presented in Chapter 9 simulates the local dynamics within a set of cells of cellular automatum. While the resistance development is sensitive to the migration of mosquitoes and parasites, inclusion of spatial dynamics is important to assess malaria policies. Such a model would be interesting while it includes local dynamics (eg. resistant development) to simulate adaptation and takes into account spatial characteristics (eg. migration, land use) which influence the adaptation process.

One step further than such a malaria model for a less developed region would be the inclusion of various other processes which are important for the local change influencing land use changes, erosion, land productivity, health, suitability of agricultural and industrial activities. For more developed regions, an interactive model to assess the competition of housing, working, recreation, and nature is more a topic of sustainable spatial development, for example, for the Green Heart of the Netherlands. Such models enable us to analyze the interactions of agents' activities and their environment, the interactions of local changes and global change and may serve as a leaming environment in supporting decision-making by identifying policies to reduce the pressures on the local and global system, and by assessing the impacts of local and global change.

To summarize, it can be concluded that the rapid development of computers and (mathematical) methods provide tools which may support the decision-making for complex societal problems. In this dissertation, I have given some possible applications of these new developments which are less limited to mathematical theories, but which try to integrate methods and theories from various disciplines, in order to address the problems of uncertainty, surprise, and evolutionary change. I hope these exercises are modest little steps towards a better understanding of global change and sustainable. development. 



\section{References}

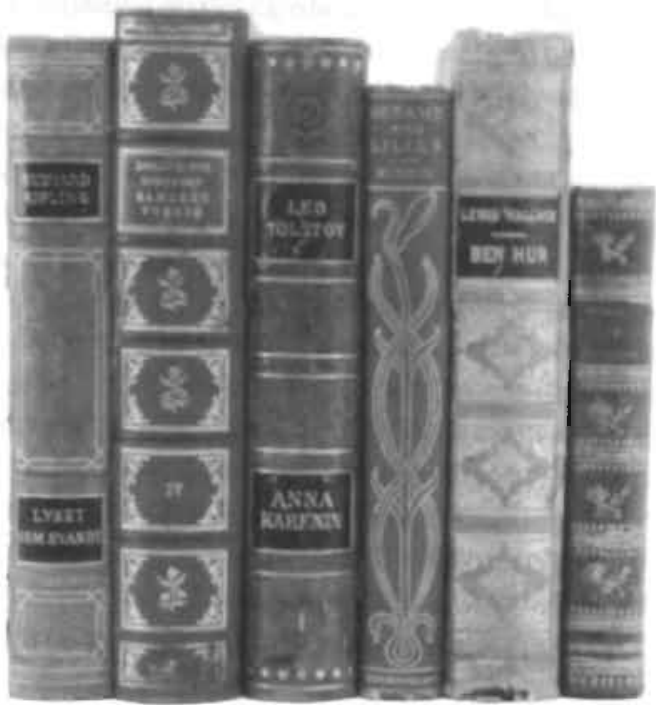


AGGG (1990), Targets and Indicators of Climate Change, Rijsberman and Swart (eds.), Report of Working Group II of the Advisory Group on Greenhouse Gases (AGGG), Stockholm Environmental Institute, Stockholm, Sweden.

Alcamo, J. R. Shaw and L. Hordijk (eds.) (1990), The RAINS Model of Acidification: Science and Strategies in Europe, Kluwer Academics Publishers, Dordrecht, the Netherlands.

Alcamo, J. (ed.) (1994), Integrated Modelling of Global Climate Change: IMAGE 2.0, Special Issue of the Journal of Water, Air and Soil Pollution.

Alcamo, J., M. Krol and M. Posch (1995), An Integrated Analysis of Sulphur Emissions, Acid Deposition and Climate Change, Water, Air \& Soil Pollution, to appear.

Alcamo, J. and E. Kreileman (1996), Emission Scenarios and Global Climate Protection Global Environmental Change, to appear.

Allen, P.M. (1990), Why the Future is not what it was: New Models of Evolution, Futures, July/August, 555-570.

Anderson, P., K.J. Arrow, and D. Pines (1988), The Economy as an Evolving Complex System, Addison Wesley, Redwood City, CA.

Anderson, R.M. and M.M. May (1991), Infectious Diseases of Humans: Dynamics and Control, Oxford University Press, New York, U.S.A.

Anderson, S.H., R.E. Beiswenger and P.W. Purdom (1993), Environmental Science, Fourth Edition, Macmillan Publishing Company, New York, USA.

Armentano, T.V. and C.W. Ralston (1980), The Role of Temperate Zone Forest in the Global Carbon Cycle, Canadian Journal of Forest Research 10: 53-60.

Aron, L.A. and R.M. May (1982), The Population Dynamics of Malaria, In: Anderson, R.M. (ed.), The population dynamics of infectious diseases: theory and applications", 139-179. Chapman and Hall, London, U.K.

Aron, J.L. and B.A. Silverman (1994), Models and Public Health Applications, Parasitic and Infection Diseases, 73-81.

Arrhenius, S. (1896), On the Influence of the Carbonic Acid in the Air upon the Temperature of the Ground, Philosophical Magazine 41, no. 251, 237-277.

Arrow, K.J. (1962), The Economic Implications of Learning-by-doing, Review of Economic Studies 29:155-173.

Arthur, W.B. (1990), Positive Feedbacks in the Economy, Scientific American, February, 92 99.

Arthur, W.B. (1994), Increasing Returns and Path Dependence in the Economy, The University of Michigan Press, USA.

Bailey, N.T.J. (1982), The Biomathematics of Malaria, Griftin, London, U.K.

Begon, M, J.L. Harper and C.R. Townsend (1990), Ecology: Individuals, Populations and Communities, Second Edition, Blackwell Science, Cambridge, USA.

Beightler, C.S., D.T. Phillips, D.J. Wilde (1979), Foundations of Optimization, 2nd. ed., Englewoods Cliffs, N.J: Prentice-Hall. 
Boyd, M.F. (1949), Epidemiology: Factors Related to the Definitive Host, In: Boyd, M.F. (ed.), Malariology (volume I)', 608-697. W.B. Saunders Company, Philadelphia and London, USA.

Bolin, B. and R.B. Cook (1983), The Major Biogeochemical Cycles and their Interactions, SCOPE 21, John Wiley \& Sons, New York, U.S.A.

Bollen, J.C., A.M.C. Toet and H.J.M. de Vries (1996), Evaluation of the Cast-Effectiveness of Implementing $\mathrm{CO}_{2}$ Emission Reduction Strategies in different Regions, Global Environmental Change, to appear.

Bongaarts, J. (1994), Population Policy Options in the Developing World, Science, No. 263, pp. 771-776.

Braddock, R.D., Filar J.A., Zapert R., Rotmans J. and den Elzen M.G.J. (1995), The IMAGE Model as a Mathematical System, Applied Mathematical Modelling, Vol. 18 May, 234-254.

Bradley, D.J. (1993), Human Tropical Diseases in a Changing Environment, In: Environmental change and human health, CIBA Found Symp 175: 147-170.

Brecke, P. (1993), Integrated Global Models that run on Personal Computers, Simulation 60, no. 2 .

Brooke, A., D. Kendrick and A. Meeraus (1988). GAMS: A User's Guide, Scientific Press, Redwood City, Califormia, USA.

Brown, L.R. (1995), State of the World 1995, EarthScan, London, UK.

Brown, L.R. (1996), State of the World 1996, EarthScan, London, UK.

Bruce-Chwatt, L.J. (1980), Essential Malariology, Heinemann Medical, London, U.K.

Bulatao, R.A., E.Bos, P.W. Stephens and M.T.Vu (1990), World Population Projections 1989-90 Edition: Short and Long-Term Estimates, The John Hopkins University Press, Baltimore (published for the World Bank).

Burniaux, J.M., J.P. Martin, G. Nicoletti and J.O. Martins (1991), GREEN: A Multi-Region Dynamic General Equilibrium Model for Quantifying the Costs of Curbing $\mathrm{CO}_{z}$ Emissions: A Technical manual, OECD Department of Economics and Statistics, Working Paper No. 89, Paris, 1991.

Butcher, S.S., R.J. Charlson, G.H. Orians and G.V. Wolfe (1992), Global Biogeochemical Cycles, Academic Press, London.

Carson, R. (1962), Silent Spring, Penguin Books, London.

Clark, W.C and R.E. Munn (eds.) (1986), Sustainable Development of the Biosphere, International Institute for Applied Systems Analysis (IIASA), Cambridge University Press, London, U.K.

Clyde, D.F. (1987), Recent Trends in the Epidemiology and Control of Malaria, Epidemiologic Reviews 9, 219-243.

Colby, M.E. (1991), Environmental Management in Development: the Evolution of Paradigms; Ecological Economics, no. 3, pp.193-213. 
Collet, H.N. and M.S. Lye (1987), Modelling the Effect of Intervention on the Transmission of Malaria in East Malaysia, Statistics in Medicine, 6, 853-861.

Comins, H.N. (1977), The Development of Insecticide Resistance in the Presence of Migration, Journal of Theoretical Biology, 64, 177-197.

Costanza, R., L. Wainger, C. Folke and K.G. Mäler (1993), Modeling Complex Ecological' Economic Systems: Toward an Evolutionary, Dynamic Understanding of People and Nature, BioScience, Vol. 43, No. 8, pp. 545-555.

Costanza, R. (1996), Managing the DICE model, Environmental Science and Technology, to appear.

Coward, H. and T. Hurka (1993), Ethics and Climate Change: The Greenhouse Effect, Wilfrid Laurier University Press, Waterloo, Canada.

Curtis, C.F. and L.N. Otoo (1986), A Simple Model to the Build-up of Resistance of Muxtures of Anti-Malarial Drugs, Trans. R. Soc. Med. Hyg., 80, 889-892.

Darmstadter, J. (1971), Enengy in the World Economy, Johns Hopkins University Press, Baltimore and London.

Darwin, C. (1859), On the Orgin of Species.

Davis, L. (Ed.) (1987), Genetic Algorithms and Simulated Annealing, London: Pitman.

De Bruin, A.J., P.J. de Vink and J.J. van Wijk (1996), $M^{\prime}$ - A Visual Simulation Tool, in Simulation in the Medical Sciences, The Society for Computer Simulation, San Diego, 181-186.

De Greene, K.B. (1993), A Systems-based Approach to Policymaking, Kluwer Academic Publishers, Boston/Dordrecht/London.

Den Elzen, M.G.J. (1993), Global Environmental Change: an Integrated Modelling Approach, $\mathrm{PhD}$ Thesis, International Books, Utrecht, the Netherlands.

Den Elzen, M.G.J., M.A. Janssen, J. Rotmans, R.J. Swart and H.J.M. de Vries (1992), Allocating Constrained Global Carbon Budgets: Interregional and Intergenerational" Equity for a Sustainable World, International Journal of Global Energy Issues, Special Issue on Energy and Sustainable Development, Vol. 4, No. 4, pp. 287-301

Den Elzen, M.G.J., A.H.W. Beusen and J. Rotmans (1995), Modelling Global Biogeochemical Cycles: an Integrated Assessment Approach, Globo-report series no. 7. RIVM, Bilthoven, the Netherlands.

Detinova, T.S. (1963), Méthodes a appliquer pour classer par groupes d'âge les diptères présentant une importance médicale, World Health Organization, Geneva, Switzerland.

Detwiler, R.P., C.A.S. Hall and P. Bodgonoff (1985), Land Use Changes and Carbon Exchange in the Tropics, II. Estimates for the Entire Region, Environmental Management 9: 335-344.

Detwiler, R.P. and C.A.S. Hall (1988), Tropical Forest and Global Carbon Cycle, Science 239: $42-47$. 
De Vries, H.J.M. (1989), Sustainable Resource Use - An Inquiry into Modelling and Planning, University of Groningen (RUG-IVEM), Groningen, the Netheriands.

De Vries, H.J.M (1994), Environmental Utilisation Space in Model Worlds (in Dutch), Milieu, Vol. 9, no. 5, 236-243.

De Vries H.J.M. and R. Van Den Wijngaart (1995), The Targets/LMage Enengy Model (TIME), GLOBO Report Series no. 16, RIVM, Bilthoven, the Netherlands.

De Vries H.J.M. and M.A. Janssen (1996), Global Energy Futures: An Integrated Perspective with the TIME Model, GLOBO Report Series no. 17, RIVM, Bilthoven, the Netherlands.

Dickinson, R.E (1986), How will Climate Change? The Climate System and Modelling of Future Climate, In: 'The Greenhouse Effect, Climate Change and Ecosystems', Bollin, B, B.R. Doôs, J. Jäger and R.A. Warrick (eds.), SCOPE 13, pp. 206-270, John Wiley and Son, Chichester, UK.

Dietz, K., L. Molineaux and A. Thomas (1974), A Malaria Model Tested in the African Savannas, Bulletin of the World Health Organization 50: 347-357.

Dotto, L. (1994), Ethical Choices and Global Greenhouse Warming, The Calgary Institute for the Humanities, Calgary, Canada.

Douglas, M. and A. Wildavsky (1982), Risk and Culture: An Essay on the Detection of Technical and Environmental Dangers, University of California Press, Berkley, USA.

Dowlatabadi, H. and G.M. Morgan (1993a), Integrated Assessment of Climate Change, Science 259, 1813-1814.

Dowlatabadi, H. and G.M. Morgan (1993b), A Model Framework for Integrated Studies of the Climate Problem, Energy Policy, 209-221.

Durand, J.D. (1967), The Modern Expansion of World Population, Proceedings of the American Philosophical Society, Vol. 111, No. 3: 136-159

Edmonds, J, and M. Wise (1995), Sustainable Development and Climate: Stabilizating Atmospheric $\mathrm{CO}_{2}$, UNU Conference on Sustainable Future of the Global System, Tokyo, 16-18 October 1995.

Ellis, J.E. and P.D. Roberts (1982), Measurement and Modelling Trade-offs for Integrated System Optimization and Parameter Estimation, Large Scale Systems 3, 191-204.

Engelen, G. R. White, I. Uljee and P. Drazan (1995), Using Cellular Automata for Integrated Modelling of Socio-Environmental Systems, Environmental Monitoring and Assessment, 34, 203-214.

Epstein, L.M and R.L. Axtell (1995), Growing Artificial Societies: Social Science from the Bortom-up, Brookings Institution, Washington, USA.

Ewald, P.W. (1994), Evolution of Infection Disease, Oxford University Press:

FAO (Food and Agriculture Organization of the United Nations) (1988), An Interim Report on the State of the Forest Resources in the Developing Countries, FAO, Rome.

FAO (Food and Agriculture Organization of the United Nations) (1991), Forest Resources Assessment 1990 Project, Forestry, No. 7, FAO, Rome. 
Farman, J.C., B.G. Gardiner and J.D. Shanklin (1985), Large Losses of Ozone in Antarctica Reveal Seasonal $\mathrm{ClO}_{x} / \mathrm{NO}_{x}$ Interaction, Nature 315, 207-210.

Faucheux, S. and G. Froger (1995), Decision-making under Environmental Uncertainty, Ecological Economics, Vol. 15, 29-42.

Ferreira, P.A.V. and T.C.D. Borges (1994), System Modelling and Optimization Under Vector-Valued Criteria, Automatica, Vol. 30, No. 2, pp. 331-336

Filar, J.A., P.S. Gaertner and M.A. Janssen (1996), An Application of Optimization to the Problem of Climate Change, in State of the Art of Global Optimization: Computational Methods and Applications by C.A. Floudas and P.M. Pardalos (eds.), Kluwer Academic Publishers, Boston (to appear).

Fisher, A. (1988), One model to fit all, Mosaic 19, no. 34, 53-59.

Foell, W.K., M. Amann, G. Carmichael, M. Chadwick, J-P. Hetteling, L. Hordijk and D. Zhao (eds.) (1995) RAINS-Asia: An Assessment Model for Airr-Pollution in Asia, Final Report to the World Bank.

Forest, S. (ed) (1993), Proceedings of the Fifth International Conference on Genetic Algorithms, Morgan Kaufmann Publishers, San Mateo, California.

Fraser, P., S.A. Penkett, R. Hariss, Y. Makide and E. Sanhueza (1992), Source Gases: Concentrations, Emissions and Trends, In Scientific Assessment of Ozone Depletion: 1991, WMO/UNEP, WMO Global Ozone Research and Monitoring Project, Report no. 25, Chapter 1.

Frenk, J., J. L. Bobadilla, C. Stern, T. Frejka and R. Lozano (1993), Elements for a Theory of the Health Transition, Chapter 2 in : L.C. Chen, A. Kleinman and N.C. Ware (eds.) 'Health and social change in intemational perspective', Harvard Series on population and international health, Harvard University Press, Boston.

Funtowitcz, S.O. and J.R. Ravetz (1990), Uncertainty and Quality in Science for Policy, Kluwer Academics Publishers, Dordrecht, the Netherlands.

Gardner, M. (1970), The Fantastic Combinations of John Conways New Solitaire Game Life. Scientific American, 223, 120-123.

Garrett-Jones, C. (1964), Prognosis for Interruption of Malaria Transmission through Assessment of the Mosquito's Vectorial Capacity, Nature 204, 1.173-1175.

Georghiou, G.P. and C.E. Taylor (1977), Genetic and Biological Influences in the Evolution of Insecticide Resistance, Journal of Economic Entomology, 70, 3, 319-323.

Glass, E.H., P.L. Adkinsson, G.A. Carlson, et al. (1984), Pesticide Resistance: Strategies and Tactics for Management, Washington, National Academy Press.

Goldberg, D. (1989), Genetic Algorithms in Search, Optimization, and Machine Learning, Reading. M.A.: Addison-Wesley.

Goudriaan. J. and P. Ketner (1984), A Simulation Study for the Global Carbon Cycle, including Man's Impact on the Biosphere, Climate Change 6: 167-192.

Graedel, T.E. and. P.J. Crutzen (1993), Atmospheric Change: An Earth System Perspective, W.H. Freeman and Company, New York. 
Grubb, M. (1989), The Greenhouse Effect: Negotiating Targets, Royal Institute of International Affairs.

Grubb, M. and J.K. Sebenius (1992), Participation, Allocation and Adaptability in International Tradable Emission Permit Systems for Greenhouse Control, in 'Climate Change-Designing a Tradeable Permit System', OECD, Paris.

Grubb, M. T. Chapuis, M. H. Duong (1995), The Economics of Changing Course, Energy Policy, Vol. 23, no. 4, p. 1-14.

Grubb, M. (1996), Technologies, Energy Systems, and the Timing of $\mathrm{CO}_{2}$ Emissions Abatement: An Overview of Economic Issues, Energy Policy, to appear

Grübler, A. and Y. Fuji (1991), Inter-Generational and Spatial Equity Issues of Carbon Accounts, Energy 16 (11/12): 1397-1416.

Haimes, Y.Y. and D.A. Wismer (1972), A Computational Approach to the Combined Problem of Optimization and Parameter Identification, Automatica, Vol. 8, pp. 337. 347.

Hammitt, J.K., R.J. Lempert, and M.E. Schlesinger (1992), A Sequential-decision Strategy for Abating Climate Change, Nature 357, 315-318.

Hao, W.M., M.H. Liu and P.J. Crutzen (1990), Estimates of Annual and Regional Releases of $\mathrm{CO}_{2}$ and Other Trace Gases to the Atmosphere from Fires in the Tropics, Based on FAO Statistics for the Period 1975 - 1980, in J.G. Goldammer (ed.), Fire in the Tropical Biota, Springer-Verlag, Berlin, pp. 440-462.

Hock, W., and K. Schittkowski (1983), A Comparative Performance Evaluation of 27 Nonlinear Programming Codes, Computing 30, 335-358.

Hofbauer, J. and K. Sigmund (1988), The Theory of Evolution and Dynamical Systems, Cambridge University Press, Cambridge, USA.

Holland, J.H. (1975), Adaptation in Natural and Artificial Systems, Ann Arbor, MIT University of Michigan Press.

Holland, J.H and J.H. Miller (1991), Artificial Adaptive Agents in Economic Theory, AEA papers and Proceedings, May 1991, 365-370.

Holland, J.H. (1992a), Genetic Algorithms, Scientific American, July, 44-50.

Holland, J.H. (1992b), Complex Adaptive Systems, Daedalus, Vol. 121, No. 1, 17-30.

Holling, C.S. (1977), Myths of Ecology and Energy, In Proceedings of the Symposium on Future Strategies for Energy Development, 36-49, Oak Ridge, TN: Oak Ridge Association of Universities.

Holling, C.S. (1978), The Spruce-Budworm/Forest-Management Problem, In Adaptive Environmental Assessment and Management, C.S. Holling (ed.), John Wiley \& Sons, Chichester - New York - Brisbane - Toronto.

Holling, C.S. (1986). The Resilience of Terrestrial Ecosystems : Local Surprise and Global Change, in: Clark, W.C. and R.E. Munn (eds.) 'Sustainable development of the Biosphere, Cambridge University Press/ IIASA Laxenburg. 
Holling, C.S. (1994), An Ecologist View of the Malthusian Conflict, In Population, Economic Development, and the Environment. K. Lindahl-Kiessling, and $\mathrm{H}$. Landberg (eds.), Oxford University Press, New York, pp. 79-103.

Holling, C.S., L.H. Gunderson and G.D. Peterson (1995), Comparing Ecological and Social Systems, Submitted to American Scientist.

Hope, C., J. Anderson and P. Wenman (1993), Policy Analysis of the Greenhouse Effect: An Application of the PAGE Model, Energy Policy, 327-338.

Horgan, J. (1995), From Complexity to Perplexity, Scientific American, June, pp. 74-79.

Horsfall, W.R. (1955), Mosquitoes: their Bionomics and Relation to Disease, Hafner Publishing Company, New York. U.S.A.

Houghton, R.A., J.E. Hobbie, J.M. Mellilo, B. Moore, B.J. Peterson, G.R. Shaver and G.M. Woodwell (1983), Changes in the Carbon Content of Terrestrial Biota and Soils between 1860 and 1980: A Net Release of $\mathrm{CO}_{2}$ to the Atmosphere, Ecological Monographs 53(3): 235-262.

Houghton, R.A., R.D. Boone, J.R. Fruci, J.E. Hobbie, J.M. Melillo, C.A. Palm, B.J. Peterson, G. R. Shaver, G.M. Woodwell, B. Moore, D.L. Skole and N. Myers (1987), The Flux of Carbon from Terrestrial Ecosystems to the Atmosphere in 1980 due to Changes in Land Use: Geographic Distribution of the Global Flux, Tellus 39B, 122139.

Houghton, R.A. and D.L. Skole (1990), Carbon, in B.L. Turner, W.C. Clark, R.W. Kates, J.F. Richards, J.T. Mathews and W.B. Meyers (eds.), 'The Earth as Transformed by Human Action', Cambridge University Press, pp. 393-408.

Houghton, R.A. (1991), Tropical Deforestation and Atmospheric $\mathrm{CO}_{3}$ Climate Change 19. 99-118

IIASA/WEC (International Institute for Applied System Analyses / World Energy Council) (1995), Global Energy Perspectives to 2050 and Beyond, WEC, UK.

MF (Intemational Monetary Fund) (1992), World Economic Outlook, May 1992, Washington DC.

IPCC (Intergovernmental Panel on Climate Change) (1990), Climate Change: The IPCC Scientific Assessment, Houghton, J.T., G.J. Jenkins and J.J. Ephraums (eds.), Cambridge University Press.

IPCC (Intergovernmental Panel on Climate Change) (1991), Climate Change: The IPCC Response Strategies, Island Press.

IPCC (Intergovernmental Panel on Climate Change) (1992), Climate Change 1992: The Supplementary Report to The IPCC Scientific Assessment, Houghton, J.T., B.A. Callander and S.K. Varney (eds.), Cambridge University Press.

IPCC (Intergovernmental Panel on Climate Change) (1996), Climate Change: The Scientific Assessment 1996, to appear.

Jager, W. M. B.A. Van Asselt, P. Costerman Boodt, J. Rotmans and C. Vlek (1996a), Modelling Consumer Behaviour: An Integrated Assessment Approach for Global 
Change and Sustainable Development, Globo Report Series No., RIVM, Bilthoven, the Netherlands.

Jager W, M.A. Janssen and J. De Greef (1996b), Modelling Behavioral Change: an Evolutionary Modelling Approach, RIVM, Bilthoven, the Netherlands, in preparation

Janssen, M.A. (1992), Historical and Future Regional Emission Paths of Curbon Dioxide: Allocation and Optimization Mechanisms, Master Thesis, Department of Operations Research, Erasmus University of Rotterdam.

Janssen, M.A., M.G.J. den Elzen and J. Rotmans (1992), Allocating COr-Emissions by Using Equity Rules and Optimization, RIVM, Report nr. 222901012, Bilthoven, The Netherlands.

Janssen, M.A., and J. Rotmans (1995), Allocation of Fossil CO, Emission Rights Quantifying Cultural Perspectives, Ecological Economics 13, 65-79.

Janssen, M.A., J. Rotmans, and O.J. Vrieze (1995), Climate Change: Optimization of Response Strategies, International Transactions in Operations Research, 2, 1-15.

Janssen, M.A. (1996a), Optimization of a Non-linear Dynamical System for Global Climate Change, European Journal of Operations Research, to appear.

Janssen, M.A. (1996b), Optimal Controlling Greenhouse Gases: Does Dynamics of the Climate System Matter?, submitted to Environmental and Resource Economics.

Janssen, M.A. (1996c), The Battle of Perspectives, submitted to Ecological Economics Janssen, M.A. and O.J. Vrieze (1996), Sequential Reduced-System Programming, submitted to Optimization Theory and Applications.

Janssen, M.A and W.J.M. Martens (1996a), Managing Malaria: an Evolutionary Modelling Approach, Globo Report Series no. 12, RIVM, Bilthoven, the Netherlands.

Janssen, M.A. and W.J.M. Martens (1996b), Modelling Malaria as a Complex Adaptive System, submitted to Artificial Life.

Janssen, M.A and H.J.M. de Vries (1996), Meeting Climate Change Policy Targets and the Role of Technological Change, submitted to Climatic Change.

Jastrow, R. W. Nierenberg, and F. Seitz, (1990), Scientific Perspectives on the Greenhouse Problem, The George C. Marshall Institute, Washington, USA.

Jetten, T.H. and W. Takken (1994), Anophelism without Malaria in Europe: a Review of the Ecology and Distribution of the Genus Anopheles in Europe, Agricultural University Wageningen, the Netherlands.

Johnson, W. C. and D.M. Sharpe (1983), The Ratio of Total to Merchantable Forest Biomass and Application to the Global Carbon Budget, Canadian Journal for Forest Research 13: $372-383$

Kamien, M.1. and Schwartz, N.L. (1981), Dynamic Optimization: the Calculus of Variations and Optimal Control in Economics and Management, North Holland, New York.

Kassler (1995), Energy for Development, Shell Selected Papers, Shell.

Kauffman, S.A. (1991), Antichaos and Adaptation, Scientific American, August, 64-70. 
Kauffman, S.A. (1993), The Origins of Order, Oxford University Press, New York, USA.

Kirkpatrick, S., C.D. Gelatt and M.P. Vecchi (1983), Optimization by Simulated Annealing, Science, 220 (4598), 671-680.

Krapivin, V.F. (1993), Mathematical Model for Global Ecological Investigations, Ecological Modelling 67, 103-127.

Krause, F., W. Bach and J. Koomey (1989), Energy Policy in the Greenhouse, Volume One, from Warming Fate to Warming Limit: Benchmarks for a Global Climate Convention, IPSEP.

Krause, F., J. Koomey and A. Sanstad (1995), Cutting Carbon Emissions: Burden or Benefit? , IPSEP, EI Cerrito, CA.

Kuhn, T.S. (1970), The Structure of Scientific Revolutions, Chicago, University of Chicago Press, second edition.

Kwa, C. (1984), The Relation with Nature in Cybemetic and Evolutionary Ecology, (in Dutch) in Kennis en Methode, 25-40.

Kwa, C. (1994), Modelling Technologies of Control, Science as Culture,Vol. 4, No. 20., 363391.

Langton, C.G. (ed.) (1989), Artificial Life, Santa Fe Institute Studies in the Sciences of Complexity, Proceedings part 6, Addison-Wesley, Redwood City (CA).

Lempert, R.J., M.E. Schlesinger and S.C. Bankes (1996), When We Don't Know the Costs or the Benefits: Adaptive Strategies for Abating Climate Change, Climatic Change, $33,235-274$.

Levin, A.S, T.G. Hallam and L.J. Gross (eds.) (1989), Applied Mathematical Ecology, New York: Springer-Verslag.

Levins, R. (1995), Preparing for Uncertainty, Ecosystem Health, Vol. 1, No. 1, 47-57.

Levy, S.B. (1992), The Antibiotic Paradox: How Miracle Drugs Are Destroying the Miracle, Plenum Press, New York and London.

Lindzen, R.S. (1990), Some Coolness about Global Warming, Bulletin of the American Meteorol. Society, 71, 288-299.

Lovelock, J.E. (1979), Gaja: a New Look at Life on Earth, Oxford. University Press, Oxford. Lovins, A.B. and H.L. Lovins (1991), Least-cost Climatic Stabilization, Annual Review of Energy and the Environment, Vol. 16.

Luenberger, D.G. (1984), Introduction to Linear and Non-linear Programming, AddisonWesley, Menlo Park, CA.

MacDonald, G. (1957), The Epidemiology and Control of Malaria, Oxford University Press, London, U.K.

Maier-Reimer, E. and K. Hasselmann (1987), Transport and Storage of Carbon Dioxide in the Ocean-An Inorganic Ocean-Circulation Carbon Cycle Model, Climate Dynamics, 2, 63-90.

Malthus, T., (1798), Essays on Population. 
Manne, A.S. and R.G. Richels (1991), Global CO, Emission Reductions: the Impacts of Rising Energy Costs, The Energy Journal, Vol. 12, No. 1, pp. 87-107.

Manne, A.S. and R.G. Richels (1992), Buying Greenhouse Insurance: The Economic Costs of $\mathrm{CO}_{2}$ Emission Limits, MIT Press, Cambridge, MA, USA.

Manne, A.S., R. Mendelsohn and R.G. Richels (1994), MERGE: A Model for Evaluating Regional and Global Effects of GHG Reduction Policies, in N. Nakicenovic, W.D. Nordhaus, R. Richels, and F.L. Toth (eds.), Integrative Assessment of Mitigation, Impacts, and Adaptation to Climate Change, 143-172, CP-94-0, IIASA, Laxenburg. Austria.

Marland, G., T.A. Boden, R.C. Griffin, S.F. Huang, P. Kanciruk, T.R. Nelson (1989), Estimates of $\mathrm{CO}_{2}$ Emissions from Fossil Fuel Burning and' Cement Manufacturing Based on the United Nations Energy Statistics and the U.S. Bureau of Mines Cement Manufacturing Data, NDP-030/RI, Carbon Dioxide Information Analysis Center, Oak Ridge National Laboratory, using updated figures NDP-030/R2.

Marland, G. and R.M. Rotty (1984), Carbon Dioxide Emissions from Fossil Fuels: a Procedure for Estimation and Results for 1950-1982, Tellus 36B: 232-261.

Martens, W.J.M., L.W. Niessen and J. Rotmans (1994), Climate Change and Malaria Risk: an Integrated Modelling Approach, RIVM Report no: 461502003, Bilthoven, the Netherlands.

Martens, W.J.M., L.W. Niessen, J. Rotmans, T.H. Jetten and A.J. McMichael (1995a), Potential Impact of Global Climate Change on Malaria Risk, Environmental Health Perspectives, Volume 103, no. 5, 458-465.

Martens, W.J.M., T.H. Jetten, J. Rotmans and L.W. Niessen (1995b), Climate Change and Vector-borne Diseases: a Global Modelling Perspective; Global Environmental Change, Volume 5 (3), 195-209.

Martens, W.J.M. and M.A. Janssen (1996), An Evolutionary Approach of Modelling Drug Resistance: A Case Study for Malaria, submitted to the Journal of Theoretical Biology.

Martin, P.H. and M.J. Lefebvre (1995), Malaria and Climate: Sensitivity of Malaria Potential Transmission to Climate, AMBIO, 2494), 200-207.

Maslow, A. (1966), The Psychology of Science: A Reconnaissance, Chicago, Henry Regnery. MATLAB (1992), Optimisation Toolbox: For Use with MATLAB, The Math Works Inc. 10.

Matsuoka, Y. and K. Kai, (1994), An Estimation of Climatic Change Effects on Malaria, Journal of the Global Environment 1:1-15.

Meadows, D.H., D.L. Meadows, J. Randers and W.W. Behrens (1972), The Limits to Growth, Universe Books, New York, U.S.A.

Meadows, D.L. W.W. Behrens III, D.H. Meadows, R.F. Naill, J. Randers, E.K.O. Zahn (1974), Dynamics of Growth in a Finite World, Wright-Allen Press, Cambridge, UK. 
Meadows, D.H. and J.M. Robinson (1985), The Electronic Oracle: Computer Models and Social Decisions, John Wiley \& Sons.

Meadows, D.H., D.L. Meadows and J. Randers (1991), Beyond the Limits, Earthscan Publications Ltd., London.

Miller, L.H. (1985), Global Order: Values and Power in International Politics, Boulder, Colorado, Westview Press.

Mitchell, B.R. (1981), European Historical Statistics: 1750-1975, Macmillan, Facts on File, New York.

Mitchell, B.R. (1982), International Historical Statistics: Africa and Asia, Macmillan Press LTD, London and Basingstoke.

Mitchell, B.R. (1983), International Historical Statistics: The Americas and Australia, Gale Research Co. Detroit.

Mitchell, J.F.B., T.C. Johns, J.M. Gregory and S.F.B. Tott (1995), Climate Response to Increasing Levels of Greenhouse Gases and Sulphate Aerosols, Nature, Vol. 376, 10 August, 501-504.

Molina, M.J. and F.S. Rowland (1974), Stratospheric Sink for Chlorofluoromethanes: Chlorine Atomcatalyzed Destruction of Ozone, Nature 249, 810-812.

Molineaux, L., K. Dietz and A. Thomas (1978), Further Epidemiological Evaluation of a Malaria Model, Bulletin of the World Health Organization.

Molineaux, L. (1988), The Epidemiology of Human Malaria as an Explanation of its Distribution, Including Some Implications for its Control, In: Wernsdorfer, W.H. and McGregor, I. (eds.), 'Malaria: principles and practice of malariology (volume 2)', 913-998. Churchill Livingstone, New York, U.S.A.

Molofsky, J., E.S. Menges, C.A.S. Hall, T.V. Armentano and K.A. Ault (1984), The Effects of Land Use Alteration on Tropical' Carbon Exchange, in T.N. Veziraglu (ed.), 'The Biosphere: Problems and Solutions', Elsevier Science Publishing, Amsterdam, pp. 181-184.

Moore, B., R.D. Boone, J.E. Hobbie, R.A. Houghton, J.M. Melillo, B.J. Peterson, G.R. Shaver, C.J. Vorosmarty, G.M. Woodwell (1981), A Simple Model for Analysis of the Role of Terrestrial Ecosystems in the Global Carbon Budget, in B. Bolin (ed.), 'Carbon Cycle Modelling', SCOPE 16, John Wiley \& Sons, New York, pp. 365-385.

Morgan, G.M. and M. Henrion (1990), Uncertainty: a Guide to Dealing with Uncertainty in Quantitative Risk and Policy Analysis, Cambridge University Press, New York, U.S.A.

Muirhead-Thompson, R.C. (1954), Factors Determining the True Reservoir of Infection of Plasmodium Falciparum and Wuchereria Bancrofti in a West African Village, Transactions of the Royal Society of Tropical Medicine and Hygiene 48 (3), 208-225.

Nakicenovic, N. and A. Grubler (eds.), (1991), Diffusion of Technologies and Social Behaviour, Springer-Verlag, Berlin/Heidelberg. 
Najera, J.A. (1974), A Critical Review of the Field Application of a Mathematical Model of Malaria Eradication, Bulletin of the World Health Organization, 50, 449-457.

Nájera, J.A., B.H. Liese and J. Hammer (1992), Malaria: New Patterns and Perspectives, World Bank Technical Paper number 183. The World Bank, Washington, D.C., U.S.A.

Nedelman, J. (1985), Introduction Review: Some New Thoughs about Old Malaria Models, Mathematical Biosciences 73: 159-182.

Nemhauser, G.L., A.H.G. Rinnooy Kan and M.J. Todd (1989), Optimization, Handbooks in Operations Research and Management Science, Volume 1, North Holland, the Netherlands.

Ness, G.D., W.D. Drake and S.R. Brechin (eds.) (1993), Population Environment Dynamics - Ideas and Observations, The University of Michigan Press.

Newton, 1 (1687), Principia Mathematica Philosophiae Naturalis, Royal Society

Niessen, L.W., H.B.M. Hilderink and J. Rotmans (1996), Roads to Health: Modelling the Health Transition, GLOBO report Series no. 15, RIVM report no. 461502015, RIVM, Bilthoven, the Netherlands.

Nordhaus, W.D. (1991a), To Slow or not to Slow: The Economics of the Greenhouse Effect, The Economic Journal, 101, 920-937.

Nordhaus, W.D. (1991b), A Survey of the Costs of Reduction of Greenhouse Gases, the Energy Journal, 12, 1, 37-65.

Nordhaus, W.D. (1992), An Optimal Transition Path for Controlling Greenhouse Gases, Science, Vol 258, 1315-1319.

Nordhaus, W.D. (1993), Rolling the 'DICE': An Optimal Transition Path for Controlling Greenhouse Gases, Resource and Energy Economics 15, 27-50, North-Holland.

Nordhaus, W.D. (1994), Managing the Global Commons: The Economics of Climate Change, MIT Press, Cambridge, MA, USA.

Nordhaus, W.D. and Z. Yang (1995), RICE: A Regional Dynamic General Equilibrium Model of Optimal Climate-Change Policy, February 271995.

Odén, S. (1968), The Acidification of Air and Precipitation and its Consequences in the Natural Environment, Ecology Committee Bull. No. 1, Swedish National Research Council, Stockholm, Sweden.

OECD (1989), World Energy Statistics and Balances 1971-1987, OECD, Paris.

OECD (1991a), Energy Statistics and Balances of Non-OECD Countries 1988-1989, OECD, Paris.

OECD (1991b), Energy Balances of OECD Countries 1960-1979, OECD, Paris.

OECD (1991c), Energy Balances of OECD Countries 1980-1989, OECD, Paris.

OECD (1992), Energy Balances of OECD Countries 1989-1990, OECD, Paris.

OECD (1993), Environmental Indicators: Basic Concepts and Terminology, Background Paper no. 1, by Group on the state of the Environment. In: Proceedings of the 
Workshop on indicators for use in environmental performance reviews, 1/2 February 1993, Paris.

Omran, A.R. (1971), The Epidemiological Transition: a Theory of the Epidemiology of Population Change, Milbank Memorial Fund 49 509-538.

Oreskes, N., K. Shrader-Frechette and K. Belitz (1994), Verification, Validation, and Conformation of Numerical Models in the Earth Sciences, Science 263, 641-646.

Pant, C.P. (1988), Malaria Vector Control: Imagociding, In Wernsdorfer, W.H. \& McGregor, I. (eds.), 'Malaria: Principles and Practice of Malariology (volume 2), 1173-1212, Churchill Livingstone, New York, U.S.A.

Paul, R.E.L., M.J. Packer, M. Walmsley, M. Lagog, L.C. Ranford-Carwright, R. Paru, K.P. Day (1995), Mating Patterns in Malaria Parasite Populations of Papua New Guinea, Science, Vol. 269, 1709-1711.

Peck, S.C. and T.J. Teisberg (1992), CETA: A Model for Carbon Emission Trajectory Assessment, The Energy Journal, Vol. 13, No. 1.

Peck, S.C and T.J. Teisberg (1993a), CO, Emissions Control: Comparing Policy Instruments, Energy Policy, 222-230.

Peck, S.C. and T.J. Teisberg (1993b), Global Warming Uncertainties and the Value of Information: An Analysis Using CETA, Resource and Energy Economics, Vol. 15, No. 1, 71-97.

Pepper, W., J. Leggett, R. Swart, J. Wasson, J. Edmonds and I. Mintzner (1992), Emission Scenarios for the IPCC: An Update, Unpublished Report prepared for IPCC Working Group I.

Ponting. C. (1992), A Green History of the World, London: Penguin.

Posch, M. J-P. Hettelingh, J. Alcamo and M. Krol (1996) Integrated Scenarios of Acidification and Climate Change in Asia and Europe, Global Environmental Change, to appear.

Press, W.H. B.P. Flannery, S.A. Teukolsky and W.T. Vetterling (1988), Numerical Recipes in C: The Art of Scientific Computing, Cambridge University Press, Cambridge.

Price, C. (1995), Emissions, Concentrations and Disappearing $\mathrm{CO}_{2}$, Resource and Energy Economics, Vol. 17, no. 1,87-97.

Ramaswamy, V., M.D. Schwarzkopf and K.P. Shine (1992), Radiative Forcing of Climate from Halocarbon-induced Global Stratospheric Loss, Nature 355, 810-812.

Ramsey, F.P. (1928), A Mathematical Theory of Saving, The Economic Journal, pp. 543559.

Rayner, S., (1991), A Cultural Perspective on the Structure and Implementation of Global Environmental Agreements, Evaluation Review, Vol. 15, No. 1, February 1991, 75. 102.

Riebsame, W.E. (1990), Social Perspectives on Global Climate Change, In: 'Managing the Global Commons: Decision-Making and Conflict Resolution in Response to Climate 
Change', Rayner S. (eds.), pp. 11-26, Report of the research workshop, August 1-4 1989, Knoxville Tennessee, USA.

Rōrsch, A and C de Hart (1993), Threshold 2000: Constraints and Scenarios for Sustainable Development in the Netherlands and Europe (in Dutch), Elmar, Rijswijk, the Netherlands.

Rose, A. (1992), Equity Consideration of Tradable Carbon Emission Entitlements, in 'Combating Global Warming: Study on a Global System of Tradable Carbon Emission Entitlements', UNCTAD, New York.

Ross, R. (1911), The Prevention of Malaria, (2nd edn.), Murray, London.

Rothen, S.M. (1995), The Greenhouse Effect in Economic Modelling: A Critical Survey, EAWAG, Dubendorf, Switzerland (draft).

Rotmans J. (1990), IMAGE: An Integrated Model to Assess the Greenhouse Effect, PhD Thesis, Kluwer Academic Publishers, Dordrecht, The Netherlands.

Rotmans, J. and M.G.J. den Elzen (1993), Modelling Feedback Mechanisms in the Carbon Cycle: Balancing the Carbon Budget, Tellus, no. 45B, pp. 301-320.

Rotmans, J., M.B.A. van Asselt, A.J. de Bruin, M.G.J. den Elzen, J. de Greef, H. Hilderink, A.Y. Hoekstra, M.A. Janssen, H.W. Köster, W.J.M. Martens, L.W. Niessen and H.J.M. de Vries (1994b), Global Change and Sustainable Development: A Modelling Perspective for the Next Decade, RIVM Report, 461502004, Bilthoven, the Netherlands.

Rotmans, J and H. Dowlatabadi (1996), Integrated Assessment of Climate Change: Evaluation of Models and Other Methods, in Human Choice and Climate Change: An International Social Science Assessment, by S. Rayner and E. Malone (eds.), in press.

Rotmans, J. and H.J.M. De Vries (eds.) (1996), Global Future Scenarios for Man and the Environment, Cambridge University Press, in press.

Rotty, R.M. and G. Marland (1986), Production of $\mathrm{CO}_{2}$ from Fossil Fuel Burning by Fuel Type: 1860-1982, Report NDP-006, carbon Dioxide Information Analysis Center, Oak Ridge National Laboratory, USA.

Rotty, R.M. (1987), A Look at $1983 \mathrm{CO}_{2}$ Emissions from Fossil Fuels (with Preliminary Data from 1984), Tellus 39, 203-208.

Ruthen, R. (1993), Adapting to Complexity, Scientific American, January, 110-117.

Schapira, A. (1990), The Resistance of Falciparum Malaria in Africa to 4-aminoquilines and Antifolates, Scandinavian Journal of Infectious Diseases, Supplementum. 75.

Schimel, D. I.G. Enting, M. Heimann, T.M.L. Wigley, D. Raynaud, D. Alves and U. Siegenthaler (1994), CO, and the Carbon Cycles, In J.T. Houghton, L.G. Meira Filho, J. Bruce, Hoesung Lee, B.A. Callander, E. Haites, N. Harris and K. Maskell (eds.). Climate Change 1994, Radiative Forcing of Climate Change and An Evaluation of the IPCC IS92 Emission Scenarios', Published for the Intergovernmental Panel on Climate Change, Cambridge University Press. 
Schlesinger, W.H. (1991), Biogeochemistry: an Analysis of Global Change, Academic Press, United Kingdom, London.

Schwartz, M. and M. Thompson (1990), Divided We Stand: Redefining Politics, Technology, and Social Choice, New York, Harvester Wheatsheaf.

Seitz J.L. (1995), Global Issues: An Introduction, Blackwell Publishers Inc., Cambridge, USA.

Siegenthaler, U. and $\mathrm{H}$. Oeschger (1987), Biospheric $\mathrm{CO}_{2}$ Emissions during the Past 200 Years Reconstructed by Deconvolution of Ice Core Data, Tellus 39B: 140-154.

Sterman, J.D. (1991), A Sceptic's Guide to Computer Model, in G.O. Barney, W.B. Kreutzer, and M.J. Garrett (eds.), 'Managing a Nation: The Microcomputer Software Catalog', Westview Press, Boulder-San Francisco-Oxford.

Tabashnik, B.E. (1986), Computer Simulation as a Tool for Pesticide Resistance Management, in Pesticide Resistance: Strategies and Tactics for Management, Committee on Strategies for the Management of Pesticide Resistant Pest Populations. (ed. ), National Academy Press, Washington, D.C.

Tabashnik, B.E. and B.A. Corft (1982), Managing Pesticide Resistance in Crop-Arthropod Complexes: Interactions Between Biological and Operational Factors, Environmental Entomology, 11: 1137-1144.

Tabashnik, B.E. (1990), Implications of Gene Amplification for Evolution and Management of Insecticide Resistance, Journal of Economic Entomology, Vol. 83, no. 4, 11701176.

Taylor, C.E. (1983), Evolution of Resistance to Insecticides: the Role of Mathematical Models and Computer Simulations, pp. 163-173. In G.P. Georghiou \& T. Saito (eds.). Pest resistance to pesticides, Plenum, New York.

Tayior, K.E. and I.E. Penner (1994), Response of the Climate System to Atmospheric Aerosols and Greenhouse Gases, Nature, Vol. 369., pp. 734-737.

Thompson, M., R. Ellis, and A. Wildawsky, (1990), Cultural Theory "Westview Press, Boulder, Colorado, USA.

Thompson, M. and P. Taylor, (1986) , The Surprise Game: An Exploration of Constrained Relativism, Warwick Pạpers in Management, no. 1, Institute for Management Research and Development (Coventry: University of Warwick).

Timmerman, P. (1986), Mythology and Surprise in the Sustainable Development of the Biosphere, Cambridge University Press, Cambridge, UK.

Tobler, W. (1979), Cellular Geography, in Gale, S. and G. Olsson (eds.), 'Philosophy of Geography', 379-386.

Toulmin, S. (1990), Cosmopolis. The Hidden Agenda of Modernity, New York.

Tyndall, J. (1863), On Radiation Through the Earth's Atmosphere, Philosophical Magazine, 4: 200 
Trisoglio, A., R. Swart, M. Thompson, J. Rotmans, H. Dowlatabadi, A. Rahman, and B. de Vries, (1994), Scenarios of Climate Change: Making the Human Factor Explicit, Washington: Battellę Pacific Northwest Laboratories.

Trisoglio, A. (1995), Complexity: The Challenges, Paper presented at the workshop "Risk, Policy and Complexity", IIASA, Laxenburg, 7-9 August 1995.

United Nations (UN) (1966), World Population Prospects as Assessed 1963, United Nations, New York.

United Nations (UN) (1990a), Population Prospects 1990, United Nations, New York.

United Nations (UN) (1990b), National Accounts Statistics: Analysis of main Aggregates, 1987, New York.

United Nations (1992), Framework Convention on Climate Change, United Nations, New York.

United Nations Conference on Trade and Development (UNCTAD) (1985), Handbook of International Trade and Development Statistics Supplement 1985, New York.

UNEP (United Nations Environment Programme) (1992), The World Environment 1972. 1992; Two Decades of Challenge, M.K. Tolba and O.A. El-Kholy (eds.), Chapman \& Hall, London.

Van Asselt, M.B.A., and J. Rotmans (1996), Uncertainty in Perspective: A Cultural Perspective Based Approach, Global Environmental Change, to appear.

Van Peursen, C.A. (1994), After Post-modernism. From Metaphysics until Philosophical Surrealism (in Dutch), Kok Agora/Pelckmans, Kampen, the Netherlands.

Vercelli, A. (1994), Hard Uncertainty and Environment, In: F.E. Mattei (ed.), 'Sustainable Growth and Uncertainty', Proceedings of a Symposium, January 10-11, Milan, Italy.

Von Bertalanffy, L. (1968), General System Theory, New York.

Waldrop, M. (1992), Complexity: The Emerging Science at the Edge of Order and Chaos, Simon and Schuster.

Watts, J.A. (1982), The Carbon Dioxide Question: Data Sampler, in W.C. Clark (ed.), 'Carbon Dioxide Review', pp. 457-460.

WCED (World Commission on Environment and Development) (1987), Our Common Future, Oxford, Oxford University Press.

Weaver, W. (1948), Science and Complexity, American Scientist, 36, 536-544

Wernsdorfer, W.H. (1994), Epidemiology of Drug Resistance in Malaria, Acta Tropica, 56, 143-156.

Weyant, J., O. Davidson, H. Dowlatabadi, J. Edmonds, M. Grubb, E.A. Parson, R. Richels, J. Rotmans, P.R. Shukla, R.S.J. Tol, W. Cline and S. Fankhauser (1996), Integrated Assesment, Climate Change 1995: Economic and Social Dimensions of Climate Change. Contribution of Working Group III to the Second Assessment Report of the IPCC, pp. 367-396, Cambridge University Press.

White, I.D., D.N. Mottershead and S.J. Harrison (1992), Environmental Systems: An Introductory Text, Second Edition, Chapman \& Hall, London. 
White, R. and G. Engelen (1994), Cellular Dynamics and GIS: Modelling Spatial Complexity, Geographical Systems 1(2), 237-253.

WHO (World Health Organization) (1991), Weekly Epidemiological Record, 66(22): 162.

WHO (World Health Organization) (1992), Vector Resistance to Pesticides, Fifteenth Report of the WHO Expert Committee on Vector Biology and Control, WHO Technical Report Series, 818, Geneva.

WHO (World: Health Organization) (1992b), Our Planet, Our Health, Report of the WHO Commission on Health and Environment, WHO, Geneva.

WHO (World Health Organization) (1996), The World Health Report 1996, WHO, Geneva.

Wigley, T.M.L. (1994), MAGICC: Model for the Assessment of Greenhouse-gas Induced Climate Change: User's Guide and Scientific Reference Manual, Preliminary version.

Wigley, T.M. L. (1995), A Successful Prediction?, Nature, Vol. 376, 10 August, 463-464.

Wigley, T.M.L., R. Richels and J.A. Edmonds (1996), Economic and Environmental Choices in the Stabilization of Atmospheric $\mathrm{CO}_{2}$ Concentrations, Nature, Vol. 379, 240-243

Willams, R.H. (1990), Low-cost Strategies for Coping with $\mathrm{CO}_{2}$ Emissions Limits, The Energy Journal, Vol. 11, No. 3, pp. 35-59.

Wilson, D. and J. Swisher (1993), Exploring the Gap: Top-down versus Bottom-up Analyses of the Costs of Mitigating Global Warming, Energy Policy, 249-263.

WMO (World Meteorological Organization) (1992), Scientific Assessment of Ozone Depletion: 1991, WMO/UNEP, WMO Global Ozone Research and Monitoring Project, Report no. 25.

Wolfe, P. (1959), The Simplex Method for Quadratic Programming, Econometrica, Vol.27, pp. 382-398.

World Resources Institute/Institute for Environment and Development (1991), World Resources 1990/1991, Washington.

WRR (Dutch Scientific Council for Governmental Policy) (1994), Sustained Risks: a Lasting Phenomenon, Reports to the Government no. 44, SDU Uitgeverij. The Hague, the Netherlands.

Zapert, R. (1994), Uncertainty Analysis of Enhanced Greenhouse Effect Models, PhD Thesis, University of Maryland, Baltimore County, Maryland, USA.

Zweers, W.. (1984), Nature and Culture in an Ecological Perspective, In: Milieucrises en filosofie, westers bewustzijn en vervreemde natuur', pp. 97-141, Ekologische uitgeverij, Amsterdam, the Netherlands (in Dutch). 


\section{Samenvatting.}

De activiteiten van de mens hebben de laatste decennia zo'n omvang gekregen dat de invloed ervan wereldwijd zichtbaar is. Het besef is gegroeid dat een toename van de intensiteit en schaal van de druk van menselijk handelen op het milieu kan leiden tot ernstige schade aan menselijk welzijn. Vanuit beleidsoogpunt is hierop gereageerd door internationaal een duurzame ontwikkeling na te streven. Er is geen eenduidige definitie van duurzame ontwikkeling. Een veel gebruikte interpretatie is echter: "een ontwikkeling waarbij de behoeften van de huidige bevolking kan worden bevredigd zonder dat dit ten koste gaat van de mogelijkheden van toekomstige generaties". Om zicht te krijgen op de consequenties van het menselijk handelen in relatie tot duurzame ontwikkeling wordt steeds meer gebruik gemaakt van computersimulatiemodellen, het onderwerp van dit proefschrift.

Integrated assessment ${ }^{1}$ is een multi- en interdisciplinair proces dat tot doel heeft vanuit een geïntegreerd perspectief op een transparante wijze wetenschappelijke kennis vanuit diverse gebieden te combineren, interpreteren en verduidelijken. Een instrument in dit streven is het ontwikkelen en gebruiken van integrated assessment modellen welke vereenvoudigde versies van diverse expert modellen integreren in één model om zo op een interactieve wijze mogelijke toekomstige scenario's te analyseren.

Integrated assessment modellering is een jonge wetenschappelijke stroming die nog sterk leunt op andere disciplines. De methoden en technieken die worden gebruikt voor deze modellen zijn niet alle de meest geschikte om de problematiek te beschrijven. In het onderzoek dat ten grondslag lag aan dit proefschrift zijn enkele nieuwe methoden en technieken ontwikkeld en toegepast binnen integrated assessment modellen. Deze methoden en technieken zijn gebruikt voor de volgende kwesties:

- optimalisatie. Veel (economische) integrated assessment modellen maken gebruik van optimalisatie, waarbij vergaande simplificatie van het mens-milieu systeem is aangebracht om de klassieke optimalisatietechnieken toe te kunnen passen. In dit proefschrift worden consequenties van deze vereenvoudigingen onderzocht en een alternatieve benadering verkend.

- wereldbeelden: Onzekerheid wordt vaak beschouwd als een statistisch artefact, hoewel een groot deel van onzekerheden veroorzaakt wordt door verschillende interpretaties van de wereld om ons heen. In dit proefschrift worden verschillende perspectieven c.q. wereldbeelden gebruikt om een consistente en kwalitatieve analyse te maken van subjectieve meningen omtrent het functioneren van het mondiale systeem en de consequenties hiervan voor geprefereerd beleid. Hierbij

\footnotetext{
${ }^{1} \mathrm{Er}$ is geen goede Nederlandse vertaling voot, integrated assessment.
} 
wordt gebruik gemaakt van de Culturele Theorie van Thompson et al. (1990) welke onder andere een onderscheid maakt tussen een egalitair, hierarchistisch en individualistisch wereldbeeld.

- evolutionaire modellering de huidige generatie integrated assessment modellen worden gedomineerd door een mechanistisch wereldbeeld. Een andere benadering is die vanuit een evolutionair perspectief waarbij het systeem gezien. wordt als een veranderend en aanpassend geheel van een heterogene verzameling van actoren. In dit proefschrift worden evolutionaire modelleertechnieken toegepast om de meerwaarde van het evolutionair perspectief te illustreren.

Het proefschrift is als volgt opgebouwd. Na. een inleidend hoofdstuk wordt in hoofdstuk 2 een beknopt overzicht gegeven van het brede terrein van mondiale veranderingen, waarna een inleiding volgt van integrated assessment, en in het bijzonder integrated assessment modellering. In dit hoofdstuk wordt ook een overzicht gegeven van de belangrijkste integrated assessment modellen voor klimaatverandering. In hoofdstuk 3 worden de methodologische aspecten besproken rond optimalisatie, perspectieven en evolutionaire modellering. Deze methoden worden toegepast in de hoofdstukken 4 tot en met 9.

In hoofdstuk 4 wordt het model OMEGA (Optimization Model for Economic and Greenhouse Assessment) besproken. Dit. model is een combinatie van het economisch gedeelte van het bekende optimalisatie model DICE (Dynamic Integrated model of Climate and the Economy) van de econoom Nordhaus (1992) en het klimaatsysteemgedeelte van het natuurwetenschappelijk georiënteerd simulatiemodel IMAGE 1.0 (Integrated Model to Assess the Greenhouse Effect) (Rotmans, 1990). Door experimenteren met het oorspronkelijke DICE model en het verkregen OMEGA model kunnen de consequenties van een zeer vereenvoudigde beschrijving van het klimaatsysteem zoals in DICE gebruikt wordt, worden geanalyseerd. Doordat het klimaatsysteem in DICE niet veel meer beschrijft dan een trendextrapolatie van historische veranderingen in de atmosfeer is het model niet geschrikt om trendbreuken in emissies voldoende bevredigend te beschrijven. Om het optimalisatieprobleem met OMEGA op te lossen zijn vier soorten optimalisatietechnieken, gebruikt waaronder een nieuw ontwikkelde techniek SRSP (Sequential Reduced-System Programming) waarvan convergentie eigenschappen worden bewezen. Dit hoofdstuk illustreert dat optimale oplossingen kunnen worden verkregen voor modellen met een procesgeoriënteerde beschrijving van het klimaatsysteem en dat de verkregen oplossingen aanzienlijk kunnen verschillen met modellen met een trend georiênteerde beschrijving van het klimaatsysteem.

Een beknopte beschrijving van de achtergronden filosofie en modules van het mondiale, integrated assessment model TARGETS (Tool to Assess Regional 304 
and Global Environmental and health Targets for Sustainability) wordt gegeven in hoofdstuk 5. TARGETS integreert op een hoog aggregatieniveau het mens-milieu systeem, waarbij zowel verticale (oorzaak-gevolg) als horizontale (subsystemen) integratie wordt onderscheiden. TARGETS bestaat uit 5 modules: een bevolking en gezondheidsmodel; een energiemodel; een element-cycli-model, een watermodel en een landmodel. Geïntegreerd beschrijven ze op abstract niveau de consequenties van economische en technologische ontwikkelingen op de toestand van het milieu en de gezondheid van de mens. De energie module en de element cycli module van het TARGETS model zijn in hoofdstuk 6 gebruikt voor een aantal optimalisatie georiënteerde experimenten. Gebruikmakend van een genetisch algoritme (welke probleemoplossingen met elkaar laat concurreren) wordt geanalyseerd wat kosteneffectieve maatregelen zijn om klimaatveranderingsdoelstellingen te bereiken. Daarnaast wordt de invloed van technologische ontwikkeling op de aard en omvang van de maatregelen bekeken. Aan de hand van vier verschillende scenario's voor technologische ontwikkeling blijkt dat onzekerheid in de voortgang van technologische ontwikkeling zeer grote invloed heeft op het lange termijn klimaatbeleid. Echter, een forse beleidsimpuls op korte termijn is kosteneffectief om het energiesysteem zuiniger te laten zijn en het gebruik van alternatieve brandstoffen te laten toenemen. In de experimenten wordt expliciet de verzurende en koelende effecten van $\mathrm{SO}_{2}$ emissies meegenomen.

De verdeling van $\mathrm{CO}_{2}$ emissierechten tussen de verschillende wereldregio's is het onderwerp van hoofdstuk 7. Wanneer handel van emissierechten als instrument zal worden gebruikt om emissies van $\mathrm{CO}_{2}$ te reduceren, dan bepaalt de initiële verdeling van die rechten de stromen van middelen tussen de regio's. Gezien de huidige ongelijkheid in economische ontwikkeling, bevolking en historische bijdrage aan $\mathrm{CO}_{2}$ emissies kunnen verschillende perspectieven losgelaten worden op zo'n verdeling. Een egalitaire verdeelsleutel is een gelijke hoeveelheid emissierechten per persoon in het verleden, heden en de toekomst. Gegeven een toekomstig scenario of strategie van $\mathrm{CO}_{2}$ emissies, kunnen de huidige emissieschulden alswel de toekomstige emissierechten berekend worden. Noord Amerika en West Europa hebben de hoogste emissieschulden terwijl emissierechten in ontwikkelingslanden niet in elk scenario boven het huidige niveau uitkomen. Het gebruik van diverse perspectieven (egalitair, hierarchistisch en individualistisch) om verdeelsleutels en toekomstige emissiepaden te bepalen, sluiten aan bij de kwestie dat er geen eenduidige oplossing is van dit probleem.

In hoofdstuk 8 wordt een modelbenadering gepresenteerd waarbij actoren verondersteld worden verschillende perspectieven te hebben welke kunnen veranderen als informatie over het systeem daar aanleiding toe zal geven: The Battle of Perspectives. Verondersteld wordt dat actoren overeenkomstig hun wereldbeeld 
handelen. Gebruikmakend van een eenvoudig klimaat-economie model en een genetisch algoritme om de (verandering in) perspectieven te simuleren is een casestudie uitgewerkt. Experimenten zijn uitgevoerd voor drie mogelijke systemen: werkend zoals verondersteld door de egalitair, hierarchist en de individualist. Afhankelijk van de beginsituatie en het succes van actoren te leren wordt voor ieder systeem een spectrum verkregen van mogelijke toekomstscenario's. Deze toekomsten zijn consistent vanuit het idee dat de mens zijn beleid zal bijstellen wanneer steeds verrassingen optreden $(=$ informatie komt niet overeen met de verwachtingen). Stel, bijvoorbeeld, dat verkregen informatie (temperatuurverandering) gemaskeerd wordt door een onbekend fenomeen (koeling door sulfaat aerosolen). Een late ontdekking van dit koeleffect zou kunnen leiden tot het niet halen van beleidsdoelen. Kortom, het meenemen van leergedrag geeft toekomstprojecties van een wereld van actoren met variatie in perspectieven en beperkte informatie.

In hoofdstuk 9 wordt een model gepresenteerd dat het aanpassend gedrag van de malariamuskieten en -parasieten simuleert. Hiertoe wordt een systeemdynamisch model dat de transmissie-dynamiek van parasieten tussen mens en mug beschrijft, gekoppeld met een genetisch algoritme welke de adaptatie van muskieten en parasieten simuleert. Malariamuskieten en -parasieten passen zich aan aan het gebruik van insekticiden en medicijnen zodat malaria in veel gebieden moeilijk. bestrijdbaar is. Samen met een mogelijke toename van malaria door klimaatverandering blijt malaria een van de belangrijkste infectieziekten. Dit heuristisch model is getest aan de hand van een aantal kwesties uit de literatuur zoals de invloed van migratie, initiële resistentie en het percentage muskieten en parasieten die niet door insekticiden en medicijnen bereikt worden. Het blijkt dat in laag-endemische gebieden malaria effectief bestreden kan worden, ook wanneer klimaatverandering optreedt. Echter in hoog endemische gebieden is geen effectieve. bestrijding van malaria gevonden.

In hoofdstuk 10 tenslotte, worden de bevindingen uit het onderhavige proefschrift. samengevat en geëvalueerd en mogelijk toekomstig onderzoek aangestipt. Hierbij wordt nadruk gelegd op het gebruik van verschillende modelleerparadigma's om de aspecten van mondiale veranderingen tot zijn recht te laten komen. 


\section{Curriculum Vitae}

Marco Janssen was born in Hendrik-Ido-Ambacht, the Netherlands on March 20,1969. He completed his secondary school education (VWO) at Walburg SG in Zwijndrecht in 1987. In 1987, he entered the Erasmus University, Rotterdam, where he studied econometrics and Operations Research. From June 1991 to January 1992, he was a trainee at the Center of Mathematical Methods of the National Institute for Public Health and the Environment (RIVM) in Bilthoven, the Netherlands. During his traineeship, he did some initial optimization experiments with the integrated assessment model for climate change IMAGE and developed the emission debt concept. In March 1992, he received the Dutch equivalent (Drs.) of a Masters degree in Operations Research at the Erasmus University, Rotterdam.

In April 1992, he began his Ph.D. study at the Department of Mathematics of the University of Limburg. During his whole Ph.D. period, he did research at the RIVM, where he was a member of the project "Global Dynamics and Sustainable Development". In addition to his Ph.D. research, he served as an integrator of the integrated assessment model for global change TARGETS (a Tool to Assess Regional and Global Environmental and health. Targets for Sustainability).

Since April 1996, he has been a research associate with the IMAGE team at the RIVM, working on a regionalized world energy model, a project financed by the Dutch Research Program on Global Air Pollution and Climate Change; and applying evolutionary modelling techniques within integrated assessment models. 
